\title{
Multidimensional Reacting Flow Simulations with Coupled Homogeneous and Heterogeneous Models
}

\author{
A Dissertation \\ Presented to \\ the Faculty of the School of Engineering and Applied Science \\ University of Virginia \\ In Partial Fulfillment \\ of the Requirements for the Degree of \\ Doctor of Philosophy in Mechanical and Aerospace Engineering \\ by \\ Ryan Frederick Johnson \\ September 2014
}




\section{Approval Sheet}

This dissertation is submitted in partial fulfillment of the requirements for the degree of Doctor of Philosophy in Mechanical and Aerospace Engineering

Ryan Frederick Johnson

This dissertation has been read and approved by the Examining Committee:

Prof. Hossein Haj-Hariri, Committee Chairman

Prof. Harsha K. Chelliah, Dissertation Advisor

Prof. Leonid Zhigilei

Prof. Houston G. Wood

Prof. James McDaniel

Accepted for the School of Engineering and Applied Science:

Dean, School of Engineering and Applied Science

November 2014 
The coupling of homogeneous (gas phase) and heterogeneous (solid phase) reactions is found in various phenomena, from the burning of wood in a fireplace to the ablating heat shield of the space shuttle flying at hypersonic speeds. With the goal of understanding the complex coupling mechanisms in such flows, this dissertation focuses developing a numerical model of low-Mach number reacting flows with coupled homogeneous and heterogeneous reactions. Numerical studies were performed in collaboration with the Air Force Institute of Technology, with the objective of guiding experimental design to investigate high-energy laser interactions with surfaces. The surfaces considered so far are porous carbon samples due to the well-established literature on heterogeneous reaction rates.

In this study, the reacting carbon surfaces considered were two flow configurations: parallel flow over a reacting boundary layer (flat plate) and impinging stagnation point flow (stagnation flow). Both configurations were modeled with derived-type boundary conditions for the conservation of reacting species at a surface. Rigorous interface boundary conditions for pressure and enthalpy were also developed. All multidimensional modeling was performed using a steady-state, laminar, finite volume method solver that was constructed using the OpenFOAM package with additional sub-models for finite rate chemistry, detailed transport quantities, and heterogeneous surface reactions. Surface temperatures considered ranged from 1600 to $2600 \mathrm{~K}$ and two semi-global heterogeneous reaction models were used, one for graphite surfaces and one for carbon surfaces with 25 percent porosity.

Both the flat plate and stagnation flow investigations focused on understanding the effects that momentum and surface temperatures have on the heterogeneous-homogeneous reaction coupling. These two configurations resulted in multidimensional reacting layers that are dependent on surface reactivity, free stream velocities, and geometric configuration. Reactions that contribute a negligible amount to $\mathrm{CO}$ production were identified by defining a sensitivity metric. Reactions with $\mathrm{OH}$ and $\mathrm{O}$, despite their small concentrations, contributed on the order of fifty percent in carbon monoxide production for the nonporous 
carbon surface reactions. This indicates that trace species are important for predicting carbon surface reactions, despite their small concentrations. Additionally, for the nonporous model, reactions with $\mathrm{CO} 2$ were found to contribute less than one percent to the total production of carbon monoxide. However, it was found that neglecting this reaction caused significant differences in the multidimensional reacting layer structures. These findings particular affect past researchers who neglect surface reactions based on concentration as this study concludes that all surface reactions are important in predicting carbon surface reaction layers. Additionally, for the stagnation flow configuration, several metrics were developed that gave a conclusive optimal separation distance for using the quasi-one dimensional formulation as a predictor for parabolic, tube-jets flows impinging on reacting surfaces. Beyond these contributions, this works serves as a foundation for further studies into the multidimensional modeling of coupled homogeneous-heterogeneous reactions 


\section{Acknowledgements}

\section{Professional and Academic}

There are no words for what an experience this has been. My eight years here at the University of Virginia has been extraordinary for not only my research and education but my life-experiences. Certainly, there are many people I need to thank, for without them I would never have left the small town of Catlett, Virginia to pursue a career in such a challenging and rewarding field.

I am in debt to my advisor and confidant, Professor Harsha Chelliah. He took a chance on me, was patient while he allowed me to grow, and put up with our back-and-forth discussions regarding my dissertation work and beyond. The lab referred to these as "mom and pop" chats, as it seemed like a old married couple arguing. Regardless, I highly value such "mom and pop" chats, as most graduate students do not have such a luxury. I am very lucky to have such an invaluable, experienced, and intelligent advisor who gave such consistent guidance.

I have been supported by three fellowships during my graduate career. The National Defense Science and Engineering Graduate (NDSEG) fellowship supported me for three and a half wonderful years. In addition, the Virginia Space Grant Consortium (VSGC) supplemented NDSEG for two years. This past year I had the pleasure of receiving the Paul Voight Graduate Teaching Fellowship, where I taught Partial Differential Equaiton with Professor Patrick Hopkins. Patrick, thanks for taking a chance on me, the class was inspiring and I will always appreciate your guidance. This past summer I received the Farrah Fellowship that fully supported my continued research over the summer.

I owe a good amount of my understanding in the field of numerics and combustion to my good friend and colleague, Dr. Gaetano Esposito. He helped me develop the software that is used throughout this dissertation, and allowed many discussions that expanded my knowledge on subjects both within and out of the field of combustion. He had no such obli- 
gation to me, and many times he could have turned me away but he remained consistently there to advise me.

One of the benefits of attending such a small engineering graduate school is the community of graduate students. My friend and colleague, Mohammad Rahimi, has been here with me from the beginning. We suffered together through rigorous coding failures, grew together as researchers, and never failed to complement each others work. Brendyn Sarnacki's advice on experiments and consistent support over the years helped broaden my understanding of combustion. Additionally, working with Uzma and Greg has been a pleasure, as they have let me interrupt their experiments so that I may continue my understanding of our lab's objectives. Many thanks to you all, and to my entire combustion lab for being so cooperative over the years.

This work would not have been completed if it wasn't complemented by my friend and lab intern, Alexandra VanDine. A side project to this dissertation that also provided supplemental verification tests was an investigation on counterflow flames. Alex was instrumental in that research, she gave many hours of work to make sure the project was a success. This would have taken 5 years without you, Alex, and, for that, everyone is grateful!

I am additionally in debt to those in my writing group, for proofing this document and many others. In addition, I'd like to thank Proffesors Goyne, McDaniel, Norris, Loth, and Hopkins' labs for putting up with my unannounced visits and for chatting about a variety of scientific topics. These groups of wonderfully qualified students have been and will continue to be a source of inspiration. Our department is certainly lucky to have such a strong group of students.

I am honored to have such a well revered dissertation committee. Professor Haj-Hariri was the first person that taught me fluid dynamics. His understanding of the fluid dynamics that lie at the heart of many applications was inspirational, and truly set me on a path that developed into the fluid dynamics of reacting flows. Professor McDaniel has given me nothing but support during my entire education. As my capstone advisor, he put up with 
my outbursts during our three hour weekly class and was always there to answer questions. Professor Wood has been a great advisor to me over the past 8 years. From my applied mathematics minor advisor to one of my supervisors as an instructor here at UVA, he has been a strong ally for my continued academic career. He was the person who re-introduced me to Professor Chelliah four years ago, and for that I am in debt to him. Professor Zhigilei is a legend in the field of numerical modeling and I am honored to have you serve on my committee. I chose this committee because I knew they would subject my work to the scrutiny that is expected within the scientific community. I am truly honored to have such a respective group of individuals and am excited for the discussions to come!

\section{Family and Friends}

"No society is so precious as that of one's own family" -Thomas Jefferson

I am the descendent of Irish, mother's side, and Italian, father's side, immigrants. My mother's side came to this country prior to the potato famine and resided in the foothills of the Shenandoah park for centuries. My father's side came to this country in the early $20^{\text {th }}$ century. Their sons and daughters did everything from serving our country to building interstates. All of them hard workers. All of them American patriots. All of them trying to give their children a better life.

Most likely, none of those generations would have the foresight to see that their $21^{\text {st }}$ century son would be sitting in a coffee shop and writing a dissertation on chemically reacting flows. However, I guarantee that they did know that, someday, one of their children would have the fortune to follow their dream. This is a dream; it feels like one constantly. For my family's sacrifice (sometimes, ultimate) and resilience, I am eternally grateful. May my hard work and passion forever be fueled by my obligation to you all. I am not done yet. This is just the beginning.

I would not have made it this far without the support of others. I have had many trials, some of which I saw coming and some of which no one could prepare for. In those trials 
I always had friends by my side to support and carry me through the experience. Such support is not trivial. Those people who have given me their faith and support are there because we share a connection. I wouldn't have known how to make such connections without the unquestionable love given to me by my mother, Terry. She taught me how to find my soul, and how to touch the souls of others.

My father, Richard, is responsible for teaching me what hard work is, and what great rewards come from hard work. He taught me the importance of respect, but also taught me how to stand strong in a room of opposition. I could not have made it here without doing hard work. Both in and out of the lab, I had to be persistent in reaching my goals and my dad was always there to keep my head cool.

My Grandparents, Robert and Amelia, have been behind me every step of the way. Even with their 38 direct descendants, they keep up with me and my ambitions. My Grandfather is the only grandfather I have ever known, and has always been there to talk about rocket motors and aliens. My grandmother has been there to pass the high values of love and compassion to all of her children, grandchildren, and great-grandchildren. The family that Bob and Amelia created is unbreakable, and I am so fortunate to have such a strong foundation of a family.

I would not be here without the consistent support of my Ma-Ma, Josephine. She is the reason I was able to complete my fourth year of college. She has been my constant backbone through out these years, always wise and always reachable. I love you Ma-Ma, you are my rock.

I have two sisters who love me more than I will ever understand. Amy, you are a rockstar in how far you come, and I appreciate all of the support you have given me. Virginia, I helped you take your first steps, but you have been my own crutch this past year. Thank you both for everything.

Some people go their entire lives without meeting their cousins. I, on the other hand, have been fortunate enough to not only know my cousins, but to be treated as siblings by 
them as well as children by their parents. I am in debt to their constant support, whether it be their willingness to pick up a phone in the middle of the night, or ability to stay 16 hours in a packed car together. I love you all.

Carl, you are, and will always be my best friend. Thanks for pulling me out of the mud more than you ever had to. You are family, and I love you, man. Jimi, Beteez, and Matt, thanks for staying in touch after high school and making home feel like home. Vinnie, thanks for being so supportive and realistic. Doug and the whole Dungle crew...they can hear us in Courtney. Brooks, Nam, Ashleigh, Dan, Andrew, and Yong, thanks for taking me in at Roy's Place and for being such good friends of mine. My townie friends, Sarah, Jordan, Cary, Dieds, K-Finn, Jon, and Ben, thanks for letting me hang with the cool kids, and for all the free whiskeys on hard days. Ben and Von, thanks for being the best rugged belt makers Virginia has ever known, and the best and most honest friends I could ask for. Jessica, Allen, Rodger, and Tiffany, thanks for being such awesome housemates and friends. You made home the most important thing it could be...home. Shaun, thanks for being such a constant and ridiculously good looking friend. To my Indian family, I love you all: Puja, Rajika, Vidya, Karthik, Komal, Parul, Manas, Sameera, and Tiffany. To all my college friends who have stayed connected, Ilia, Meredyth, Elise, Eliza, Zev, Hannah, Kate, Sandeep, Sanjeev, Ian, and Andre, thanks for being there to travel and talk. To the game night crew, Darius, Cody, Ory, Yousaf, Sean, and Sarah, thanks for making Mondays 1000 times better than they could have ever been. I owe all of you a life debt. May you never be afraid to call on me; I will bring the cavalry for you.

Claire, you are beautiful, one of a kind, and I am such a lucky man to have someone as patient and supportive as you. I love you!

I have the fortune of being born into a wonderful and loving family as well as having the best friends I could ever ask for. I dedicate this work to them. 
For my friends, my family, my heaven... 


\section{Contents}

List of Figures $\quad$ viii

List of Tables $\quad$ xii

List of Symbols $\quad$ xiii

1 Introduction 1

1.1 Background ............................ 1

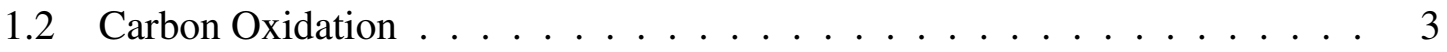

1.2.1 History and Relevance . . . . . . . . . . . . . . 3

1.2.2 The Coupling of Carbon Surfaces Reacting in Air . . . . . . . . . 4

1.2.3 Past Reacting Carbon Surface Modeling Work . . . . . . . . . 5

1.3 Fluid Dynamic Configurations . . . . . . . . . . . . . 6

1.3.1 Parallel Flow of Air Over a Reacting Flat Plate . . . . . . . . . . . 7

1.3.2 Stagnation Point Flow Over a Reacting Carbon Surface . . . . . . . 9

1.4 Supporting Experimental Efforts . . . . . . . . . . . . . . . 12

1.5 Objectives . . . . . . . . . . . . . . . . . . . 14

1.5.1 Analyze the applicability of heterogeneous kinetic models in complex geometries . . . . . . . . . . . . . . . . . . . 14

1.5.2 Assess extinction and blow-off limits of gas phase reactions . . . . 14

1.5.3 Identify optimal experimental configurations for experimental validation efforts . . . . . . . . . . . . . . . . 15

2 Formulation and Numerical Modeling $\quad 16$

2.1 The Reacting Navier Stokes Equations and Model Formulation . . . . . . . 17

2.1.1 Gas Phase Chemistry and Transport Properties . . . . . . . . . . . 20

2.2 The Finite Volume Method . . . . . . . . . . . . . . . . . . . . 22

2.3 The OpenFOAM Computational Package . . . . . . . . . . . 25

2.4 Reacting Interface Boundary Conditions . . . . . . . . . . . . . . . 26

2.4.1 Species Conservation at a Reacting Solid Surface . . . . . . . . . . 31

2.4.2 Energy Conservation at a Reacting Interface Solid Surface . . . . . 35

2.4.3 Solving for Pressure and Its Interface Conditions . . . . . . . . . . . . 40

2.4.4 Summary of Interface Conditions . . . . . . . . . . . . . . . 47

2.5 Heterogeneous Reaction Models . . . . . . . . . . . . . . . . . . 48

2.5.1 Nonporous Carbon . . . . . . . . . . . . . . . . . . 48 
2.5.2 Porous Model . . . . . . . . . . . . . . . . . . . . . . . . . . 49

2.5.3 Comparison of Heterogeneous Models . . . . . . . . . . . . . 50

2.6 Model Verification $\ldots \ldots \ldots \ldots \ldots \ldots$

2.6 .1 Chemistry . . . . . . . . . . . . . . . . 52

2.6 .2 Premixed Flame . . . . . . . . . . . . . . . . . . . 53

2.6 .3 Counterflow Flame . . . . . . . . . . . . . . . . . . 54

2.6.4 Reacting Stagnation Plate Flow Configuration . . . . . . . . . . 55

2.6.5 Grid Resolution Index . . . . . . . . . . . . . . . . . . . . . 59

3 Flow Over a Reaction Carbon Plate 62

3.1 Background . . . . . . . . . . . . . . . . 62

3.2 Computational Domain and Test Simulations _ . . . . . . . . . . 63

3.3 Results and Discussions . . . . . . . . . . . . . . . . . 65

3.3.1 Temperature Effects . . . . . . . . . . . . . . . 66

3.3.2 Residence Time Effects . . . . . . . . . . . . . . . . . . . . . . . 69

3.3.3 Heterogeneous and Homogeneous Coupling . . . . . . . . . . . 76

4 Stagnant Flow Over a Reacting Carbon Surface 83

4.1 Background . . . . . . . . . . . . . . . . 83

4.2 The Quasi One-Dimensional Formulation . . . . . . . . . . . . 85

4.3 Solution Domain and Procedure . . . . . . . . . . . . . . . . . 91 4.3.1 Optimal Experimental Configuration: Satisfying Quasi One-Dimensional Theory . . . . . . . . . . . . . . . . . . . . . 94

4.4 Results and Discussion . . . . . . . . . . . . . . . . . . . 100

4.4.1 Carbon Dioxide Flame Detachment . . . . . . . . . . . 100

4.4.2 Two-Dimensional Effects . . . . . . . . . . . . . . . . . 103

4.4.3 Heterogeneous and Homogeneous Coupling . . . . . . . . . . 117

5 Advanced Interface Conditions and Recommended Future Work 121

5.1 The Pressure Boundary Condition . . . . . . . . . . . . . . . . 121

5.2 Thermal Balance at an Interface . . . . . . . . . . . . . . . . 125

5.3 Transferable Method and Complicated Geometries . . . . . . . . . 130

6 Summary 132

$\begin{array}{ll}\text { Appendices } & 135\end{array}$

A Counterflow Paper 136

$\begin{array}{ll}\text { Bibliography } & 177\end{array}$ 


\section{List of Figures}

1.1 Reacting carbon surface with $\mathrm{C}_{s}+\mathrm{O}_{2} \rightarrow \mathrm{CO}, \mathrm{CO}+\mathrm{O}_{2} \rightarrow \mathrm{CO}_{2}$, and $\mathrm{C}_{s}+\mathrm{CO}_{2} \rightarrow 2 \mathrm{CO}$. ....................... 5

1.2 Reacting blunt carbon body subjected to stagnant and flat plate flow . . . . 7

1.3 Illustration of reacting flow over flat plate. The red material is the reacting carbon surface, lines with arrows are fluid streamlines, and vectors along the surface represent mass added by blowing velocity, $v_{n}$. . . . . . 8

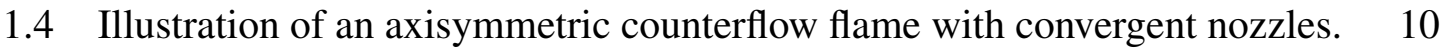

1.5 Illustration of reacting stagnation flow. The red material is the reacting carbon surface, lines with arrows are fluid streamlines, and vectors along the surface represent mass addition f . . . . . . . . . . . . . 11

1.6 Illustration of reacting stagnation flow experimental configuration . Here, the black curved material is the heated carbon, lines with arrows are fluid stream lines, $\mathrm{r}$ and $\mathrm{z}$ are coordinates, and $\mathrm{L}$ is the separation distance. Also shown is an illustration of the laser concentrically shot through the tube-jet (shown in gray). . . . . . . . . . . . . . . . . . 13

2.1 One dimensional finite volume diagram, where $i$ indicates the location in the particular dimension of interest. . . . . . . . . . . . . 23

2.2 Infinitesimal volume with interface area $\mathcal{A}_{i}$, height $\Delta n$, and surface normal

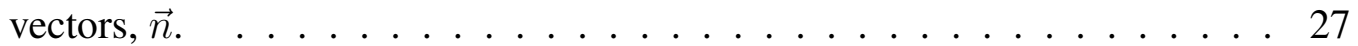

2.3 Infinitesimal volume where + is the gaseous portion and - is the solid portion. . . . . . . . . . . . . . . . . . 29

2.4 Computational cell next to reacting surface. . . . . . . . . . . . 32

2.5 Integration of species conservation at an interface using semi-implicit meth-

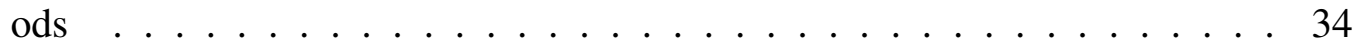

2.6 Comparisons of reaction rate ratio of porous reaction rates to nonporous reaction rates for $\mathrm{O}_{2}, \mathrm{CO}_{2}$, and water for the same composition and pressure. 51

2.7 Homogeneous combusiton at constant temperature for both OpenFOAM and SENKIN codes. Parameters are normalized to thier maximum values, ie. $T / T_{\max }, X_{H 2} /\left(X_{H 2}\right)_{\max }, X_{O 2} /\left(X_{O 2}\right)_{\max }, X_{O H} /\left(X_{O H}\right)_{\max } \ldots \ldots .52$

2.8 Spatial variation of premixed flame mole fraction concentrations for $\mathrm{H} 2$,

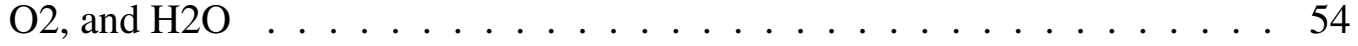

2.9 Counterflow flame temperature profiles for OpenFOAM and quasi onedimensional codes; for $a=420 \mathrm{~s}^{-1}$. 
2.10 Verification and validation tests of quasi one-dimensional and OpenFOAM FVM codes. . . . . . . . . . . . . . . . . . 57

2.11 Reacting layer profile comparion . . . . . . . . . . . . . . . 58

2.12 Decrease of maximum flame temperature with increasing strain rate for diluted non-premixed hydrogen vs. air, from both OpenFOAM and quasi one-dimensional simulations. . . . . . . . . . . . . . . . 59

2.13 Decrease in peak temperature due to numerical scheme . . . . . . . . 60

3.1 Two-dimensional computational domain used in present simulations; red area shows the reacting carbon sample is located. . . . . . . . . . . . . . 64

3.2 Two-dimensional contour plots of non-porous carbon surface reaction with freestream velocity of $40 \mathrm{~m} / \mathrm{s} \ldots \ldots \ldots 6$

3.3 Two-dimensional contour plots of porous carbon surface reaction with freestream velocity of $40 \mathrm{~m} / \mathrm{s} \ldots \ldots \ldots$. . . . . . . . . . . . . 67

3.4 Species concentrations along the reacting carbon plate for $\mathrm{CO}_{2}$ and $\mathrm{O}_{2}$ for temperatures ranging from 1800 to $2600 \mathrm{~K}$; line legends are the same for

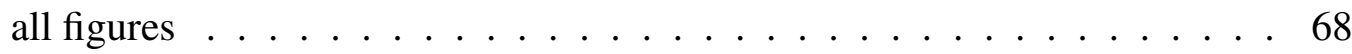

3.5 Species concentrations along the reacting porous carbon plate for $\mathrm{CO}_{2}$ and $\mathrm{O}_{2}$ for temperatures ranging from 1800 to $2600 \mathrm{~K}$; line legends are the same for all figures . . . . . . . . . . . . . . . . . . 68

3.6 Contour plots of $\mathrm{O}_{2}, \mathrm{CO}$, and $\mathrm{CO}_{2}$ for nonporous surface reaction model, surface temperature of $1800 \mathrm{~K}$, and free-stream velocity of (a) $30 \mathrm{~m} / \mathrm{s}$ and (b) $50 \mathrm{~m} / \mathrm{s} \ldots \ldots \ldots \ldots$. . . . . . . . . . . . . . . . . . . 69

3.7 Species concentrations along the reacting carbon plate for $\mathrm{CO}_{2}$ and $\mathrm{CO}$ for free-stream velocities $30,40,50 \mathrm{~m} / \mathrm{s}$ with surface temperatuer $1800 \mathrm{~K}$. . . 70

3.8 Contour plot of $R_{C / D}$ for $\mathrm{CO}_{2}$ using porous surface model, for a freestream velocity of $50 \mathrm{~m} / \mathrm{s}$ with surface temperature of $1800 \mathrm{~K}$. . . . . . . . 72

3.9 Two-dimensional contour lines for $\mathrm{CO}_{2} R_{C / D}$ number of unity for freestream velocities 30,40 , and $50 \mathrm{~m} / \mathrm{s}$ with surface temperature $1800 \mathrm{~K}$. . . 73

3.10 Terms of the $\mathrm{CO}_{2}$ conservation equation, $\frac{\rho v_{j} \partial Y_{C O 2}}{\partial x_{j}}+\frac{\partial \rho V_{j} Y_{i}}{\partial x_{j}}+w_{C O}=0$, for the nonporous model with 30 and $50 \mathrm{~m} / \mathrm{s}$ inflow velocity and surface temperature $T_{s}=1800 \mathrm{~K} \ldots \ldots \ldots \ldots$

3.11 Terms of the $\mathrm{CO}_{2}$ conservation equation, $\frac{\rho v_{j} \partial Y_{C O 2}}{\partial x_{j}}+\frac{\partial \rho V_{j} Y_{i}}{\partial x_{j}}+w_{C O}=$ 0 , for the porous model with 30 and $50 \mathrm{~m} / \mathrm{s}$ inflow velocity and surface temperature $T_{s}=1800 \mathrm{~K} \ldots \ldots \ldots \ldots 75$

3.12 Coupling of gaseous $\mathrm{O}_{2}$ and $\mathrm{CO}$ production, $\Phi_{O 2} \ldots \ldots \ldots \ldots 77$

3.13 Coupling of gaseous $\mathrm{OH}$ and $\mathrm{CO}$ production, $\Phi_{O H} \ldots \ldots \ldots$

3.14 Coupling of gaseous $\mathrm{O}$ and $\mathrm{CO}$ production, $\Phi_{O} \ldots \ldots \ldots \ldots$

3.15 Coupling of gaseous $\mathrm{O}$ and $\mathrm{CO}$ production, $\Phi_{\mathrm{H}_{2} \mathrm{O}} \ldots \ldots \ldots$

3.16 Coupling of gaseous $\mathrm{CO}_{2}$ and $\mathrm{CO}$ production, $\Phi_{\mathrm{CO}_{2}} \ldots \ldots \ldots$. . . . . 80

$3.17 \mathrm{CO}$ concentration along plate for $\mathrm{CO}_{2}$ reaction on (-) and $\mathrm{CO}_{2}$ reaction off $(-) \ldots \ldots \ldots \ldots \ldots$. . . . . . . . . . . . . . 81

3.18 Reacting layer for cases with (-) and without (--) $\mathrm{CO}_{2}$ surface reaction. . . . 81 
4.1 Reacting carbon surface stagnation flow experimental configuration, showing concentric laser (in red), and radial coordinates, $r$ and $z$. . . . . . 84

4.2 Axial velocity profiles with normalized velocity, $\bar{v}=v / v_{a x}\left(v_{a x}\right.$ is axial velocity at the axis of symmetry) and normalized radial distances, $\bar{r}=r / R$ ( $R$ is the radius). . . . . . . . . . . . . . . . 87

4.3 Two-dimensional axisymmetric domains. . . . . . . . . . . . . . 91

4.4 Axial velocity, $v_{z}$, and self-similarity function, $U(z)$ for two separation distances. . . . . . . . . . . . . . . . . 95

4.5 Response of various stagnation flow variables as a function of length . . . . 97

4.6 Response of various stagnation flow variables as a function of max parabolic velocity. . . . . . . . . . . . . . . . . . . . . . . 99

4.7 Change in mass loss rate and reaction layer profile of $\mathrm{CO}_{2}$ due to surface velocity, (a), and temperature, (b), effects. Velocity response reported here is for $T_{s}=2200 \mathrm{~K}$ and nonporous carbon. The temperature response here is for $v_{o}=1 \mathrm{~m} / \mathrm{s}$. . . . . . . . . . . . . . . . . . . 101

4.8 Two dimensional stagnation results for porous carbon model with plugflow velocity of $3 \mathrm{~m} / \mathrm{s}$. Contour colors show $\mathrm{CO}_{2}$ mole fraction profiles and white lines are stream-lines. . . . . . . . . . . . . . . . . . 102

4.9 Response of $\mathrm{CO}_{2}$ reaction layer height to two-dimensional parameter sweeps. 103

4.10 Mole fraction results for $\mathrm{CO}, \mathrm{CO}_{2}, \mathrm{O}_{2}, \mathrm{H}_{2} \mathrm{O}, \mathrm{O}$, and $\mathrm{OH}$ for the simulations using the nonporous reaction model at $T_{s}=1600 \mathrm{~K}$. . The tube region included in the simulation is not shown in these images as no reactions occurred within the tube walls. . . . . . . . . . . . . . . 105

4.11 Mole fraction results for $\mathrm{CO}, \mathrm{CO}_{2}, \mathrm{O}_{2}, \mathrm{H}_{2} \mathrm{O}, \mathrm{O}$, and $\mathrm{OH}$ for the simulations using the nonporous reaction model at $T_{s}=2400 \mathrm{~K}$. . The tube region included in the simulation is not shown in these images as no reactions occurred within the tube walls. . . . . . . . . . . . . . . 106

4.12 Mole fraction results for $\mathrm{CO}, \mathrm{CO}_{2}, \mathrm{O}_{2}, \mathrm{H}_{2} \mathrm{O}, \mathrm{O}$, and $\mathrm{OH}$ for the simulations using the porous reaction model at $T_{s}=1600 \mathrm{~K}$. . The tube region included in the simulation is not shown in these images as no reactions occurred within the tube walls. . . . . . . . . . . . . . . . . . 107

4.13 Mole fraction results for $\mathrm{CO}, \mathrm{CO}_{2}, \mathrm{O}_{2}, \mathrm{H}_{2} \mathrm{O}, \mathrm{O}$, and $\mathrm{OH}$ for the simulations using the porous reaction model at $T_{s}=2400 \mathrm{~K}$. . The tube region included in the simulation is not shown in these images as no reactions occurred within the tube walls. . . . . . . . . . . . . . . . 108

4.14 Species concentration results as function of normal distance, $\mathrm{z}(\mathrm{mm})$, for $T_{s}=[1600,2400] \mathrm{K}$ two locations, $r=0$ (dashed lines) and $r=10 \mathrm{~mm}$ (solid lines), using the nonporous carbon surface model. . . . . . . . . . 109

4.15 Species concentration results as function of normal distance, $\mathrm{z}(\mathrm{mm})$, for $T_{s}=[1600,2400] \mathrm{K}$ two locations, $r=0$ (dashed lines) and $r=10 \mathrm{~mm}$ (solid lines), using the porous carbon surface model. . . . . . . . . . . . 109

4.16 Nonporous model consumed surface species mass fractions for $T_{s}=[1600,1800,2000,2200,240$

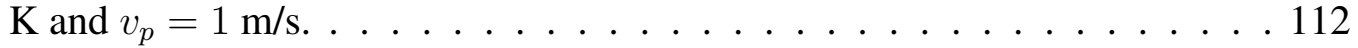

4.17 Porous model consumed surface species mass fractions for $T_{s}=[1600,1800,2000,2200,2400]$

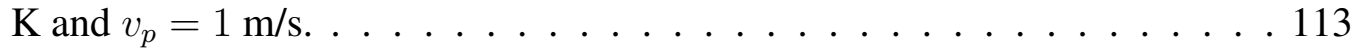


4.18 Terms of the $\mathrm{CO}_{2}$ species conservation equation, convective (shown with $-\diamond-$ ), chemical source ( shown with - $\square$-), and diffusion (shown with -०-). Data is sample normal to the plate at locations $\mathrm{r}=0 \mathrm{~mm}$ (shown with dashed line, --) and $\mathrm{r}=10 \mathrm{~mm}$ (shown with solid line, -) . . . . . . . . . . . . . 114

4.19 Terms of the $\mathrm{CO}_{2}$ species conservation equation, convective (shown with $-\diamond-$ ), chemical source ( shown with $-\square$-), and diffusion (shown with -०-) for $T_{s}=1850 \mathrm{~K}$. Data is sampled normal to the plate at locations $\mathrm{r}=0 \mathrm{~mm}$ (shown with dashed line, --) and $\mathrm{r}=10 \mathrm{~mm}$ (shown with solid line, -). Also shown is $\mathrm{CO}_{2}$ reaction layer at the two separate radial locations, $r=0$ and $10 \mathrm{~mm}$. At $r=0 \mathrm{~mm}$ the flame is attached and at the $r=10 \mathrm{~mm}$ the flame is detached. . . . . . . . . . . . . . . . . . . . . . . . 116

4.20 Mass loss rate for $T_{s}=[1600,1800,2000,2200,2400] \mathrm{K}$ for both porous and nonporous models. . . . . . . . . . . . . . . . . 117

4.21 Homogeneous-heterogeneous coupling metric, $\phi$, for each reactant species for both porous, (a), and nonporous (b) surface reaction models. . . . . . . 118

4.22 Nonporous mass loss rate with $\mathrm{C}_{s}+\mathrm{CO}_{2}$ surface reaction included (shown with solid line) and with the reaction neglected (shown with dashed, --,

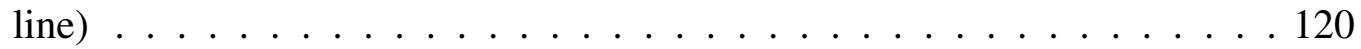

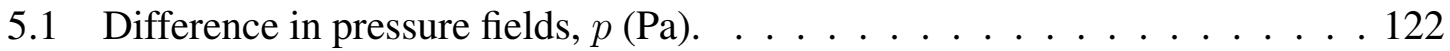

5.2 Difference in radial velocity field, $v_{r}(\mathrm{~cm} / \mathrm{s}) \ldots \ldots \ldots \ldots$

5.3 Difference in axial velocity field, $v_{z}(\mathrm{~cm} / \mathrm{s}) \ldots \ldots \ldots . \ldots . \ldots . \ldots 124$

5.4 Difference between stagnation flow simulations with pressure boundary condition calculated from Equation 5.1 and pressure gradient set to $\frac{\partial p}{\partial n}=0.124$

5.5 Results with Equation 5.2 used to determine surface temperature. . . . . . . 126

5.6 Mass loss rate for three different thermal interface conditions: (1) Gaussian $\mathcal{L}(r),(2)$ constant $\mathcal{L}$, and (3) constant surface temperature, $T_{s}$, fixed to the calculated axis of symmetry temperature from (1). . . . . . . . . . . . . . . 129

5.7 $\mathrm{CO}_{2}$ contour for a reacting carbon sphere in air heated sphere. White lines show stream lines with flow direction in the towards the right. . . . . . . 131 


\section{List of Tables}

2.1 The specific reaction-rate constants for the $\mathrm{CO} / \mathrm{H}_{2} \mathrm{O} / \mathrm{O}_{2}$ mechanism from Yetter et al. in the form $k_{j}=B_{j} T^{\alpha_{j}} \exp \left(-E_{j} / R T\right) . \ldots \ldots \ldots \ldots 21$

2.2 Conservation equations at a reacting carbon interface . . . . . . . 47

2.3 The heterogeneous reaction rate parameters of non-porous carbon as compiled by Bradley et al. . . . . . . . . . . . . . . . . . . . . 48

2.4 The heterogeneous rate constants of porous carbon from Chelliah et al. . . 49

3.1 Numerical boundary conditions imposed on five surfaces identified in Fig.

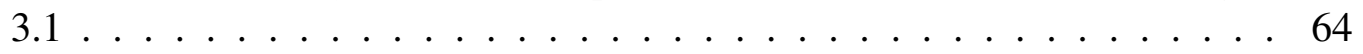

3.2 Parametric values for free-stream velocity and surface temperature explored in the present study. . . . . . . . . . . . . . . . . 65

4.1 List of boundary conditions for Figure 4.7's computational domains. . . . . 92

4.2 Table of experimental considerations for porous and nonporous surfaces with $T_{s}=[1600,2400] \mathrm{K}$ surface temperatures. . . . . . . . . . . 111

5.1 Terms of thermal interface condition for stagnation flow configuration with Gaussian laser effluence profile, $\mathcal{L}(r)$, (average effluence is $100 \mathrm{~W} / \mathrm{cm}^{2}$ ) and parabolic velocity profile with $v_{p}=1 \mathrm{~m} / \mathrm{s} \ldots \ldots \ldots \ldots 127$ 


\section{List of Symbols}

All symbols are in the order that they appear in each chapter. Any repeated definitions are not redefined. Each chapter has its own dictionary as some variable names are changed.

\section{Chapter 1}

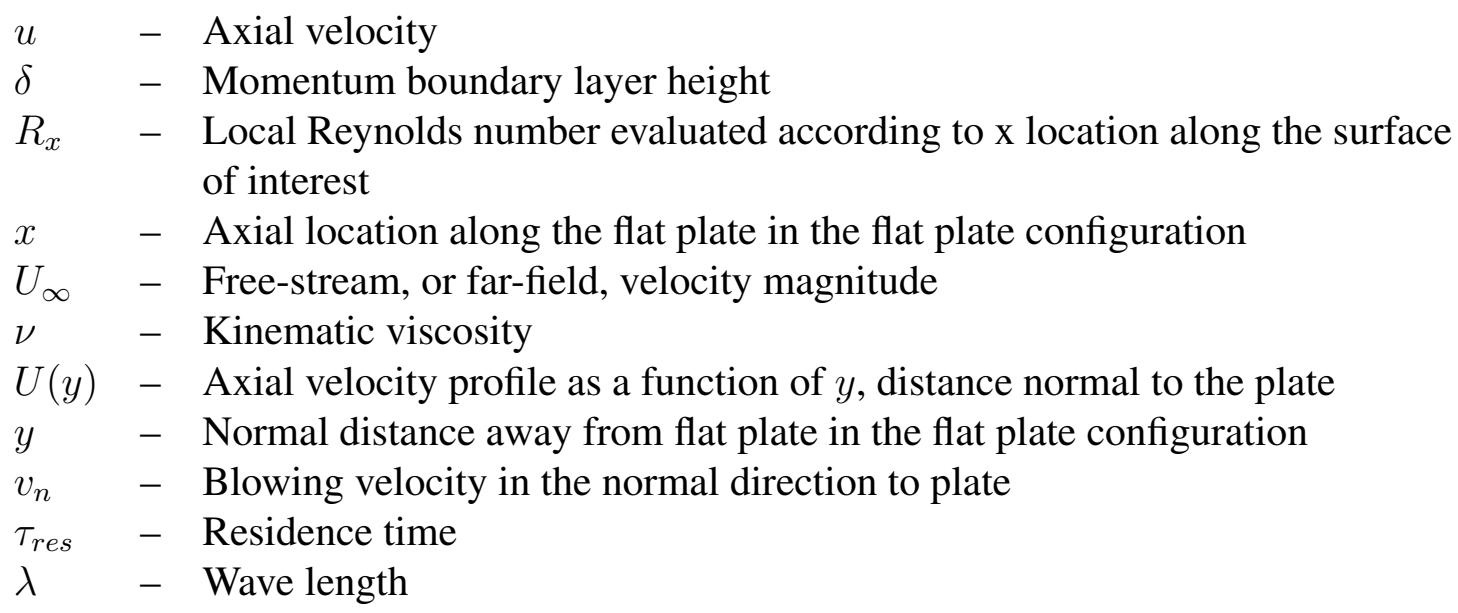




\section{Chapter 2}

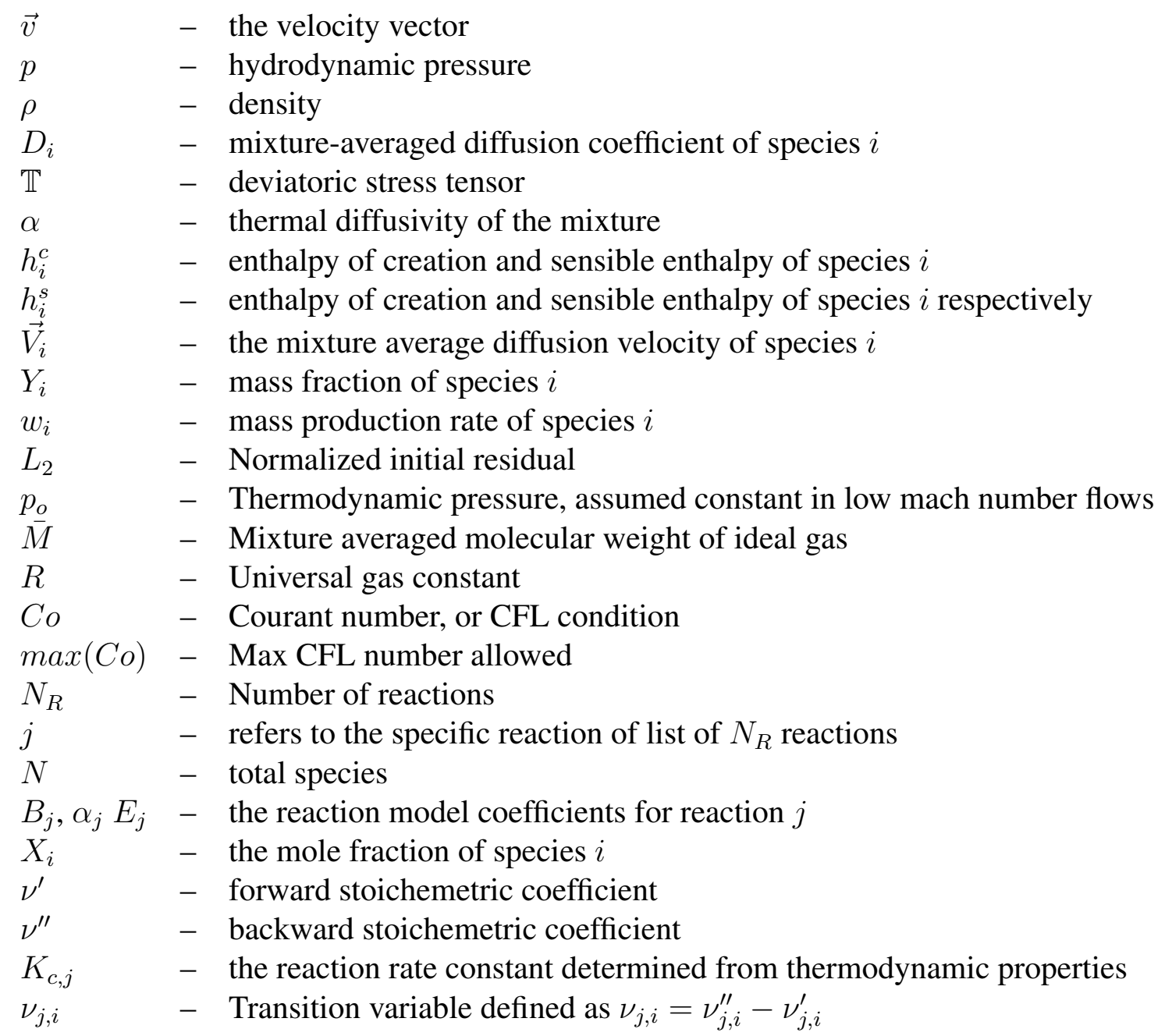




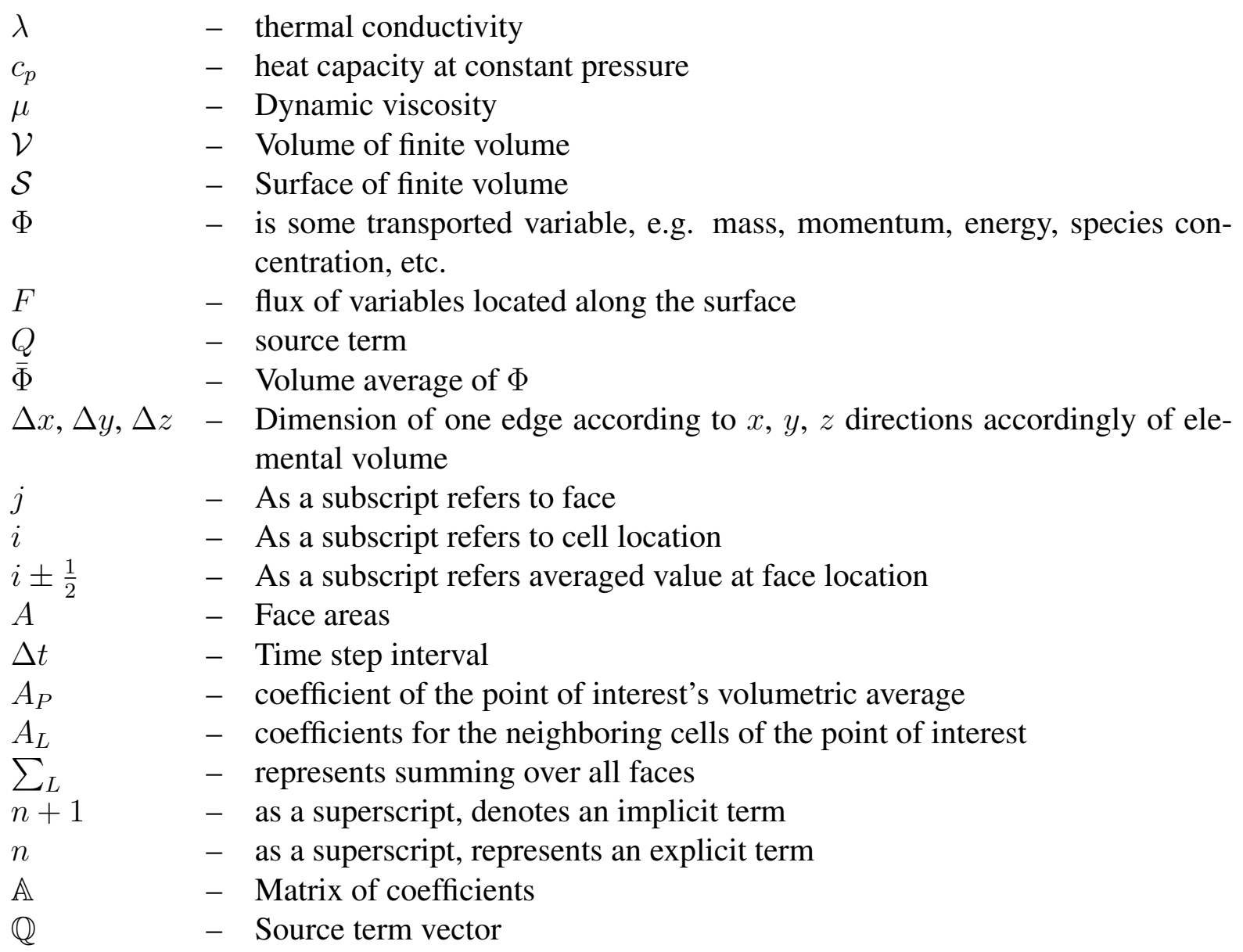




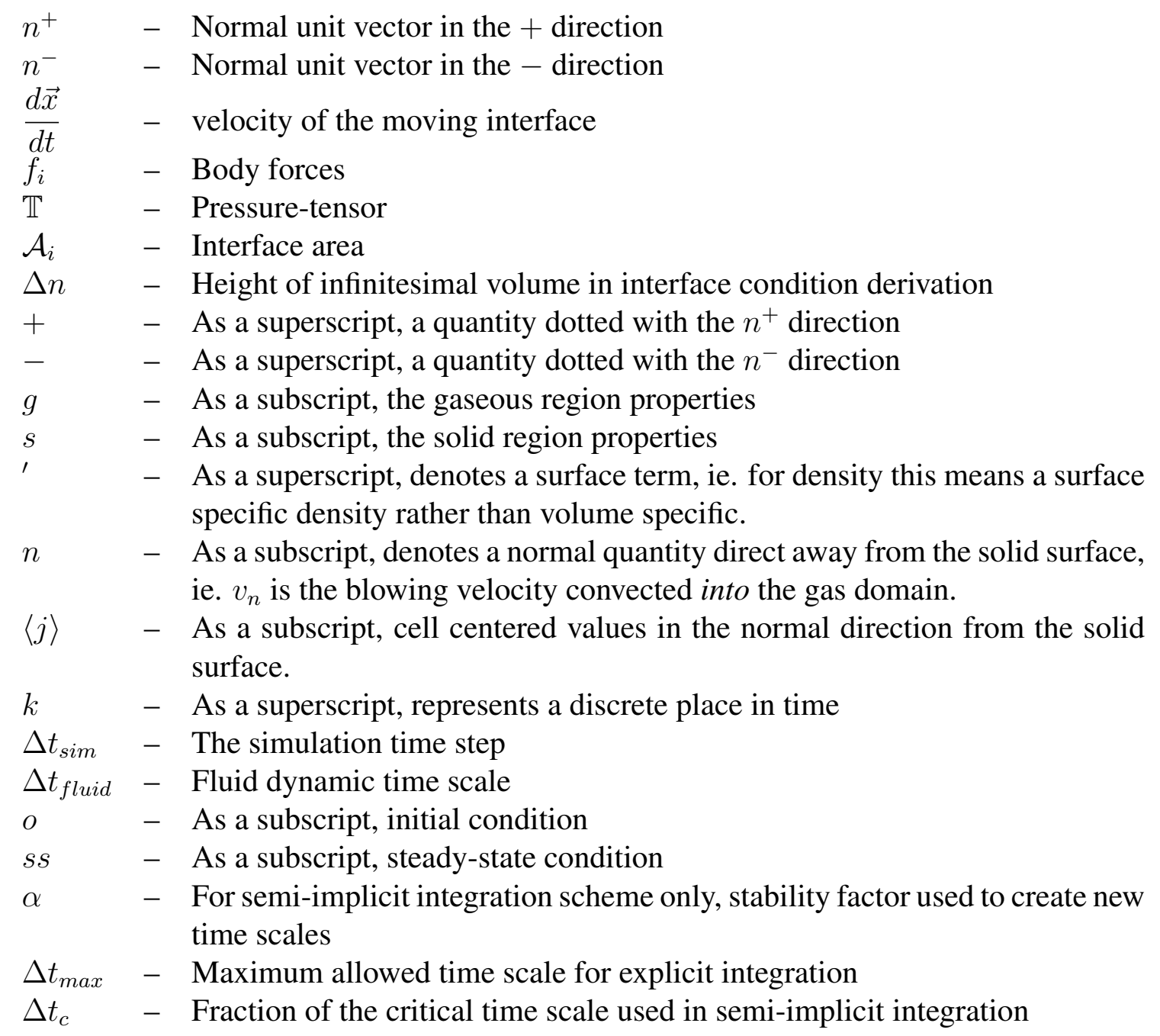




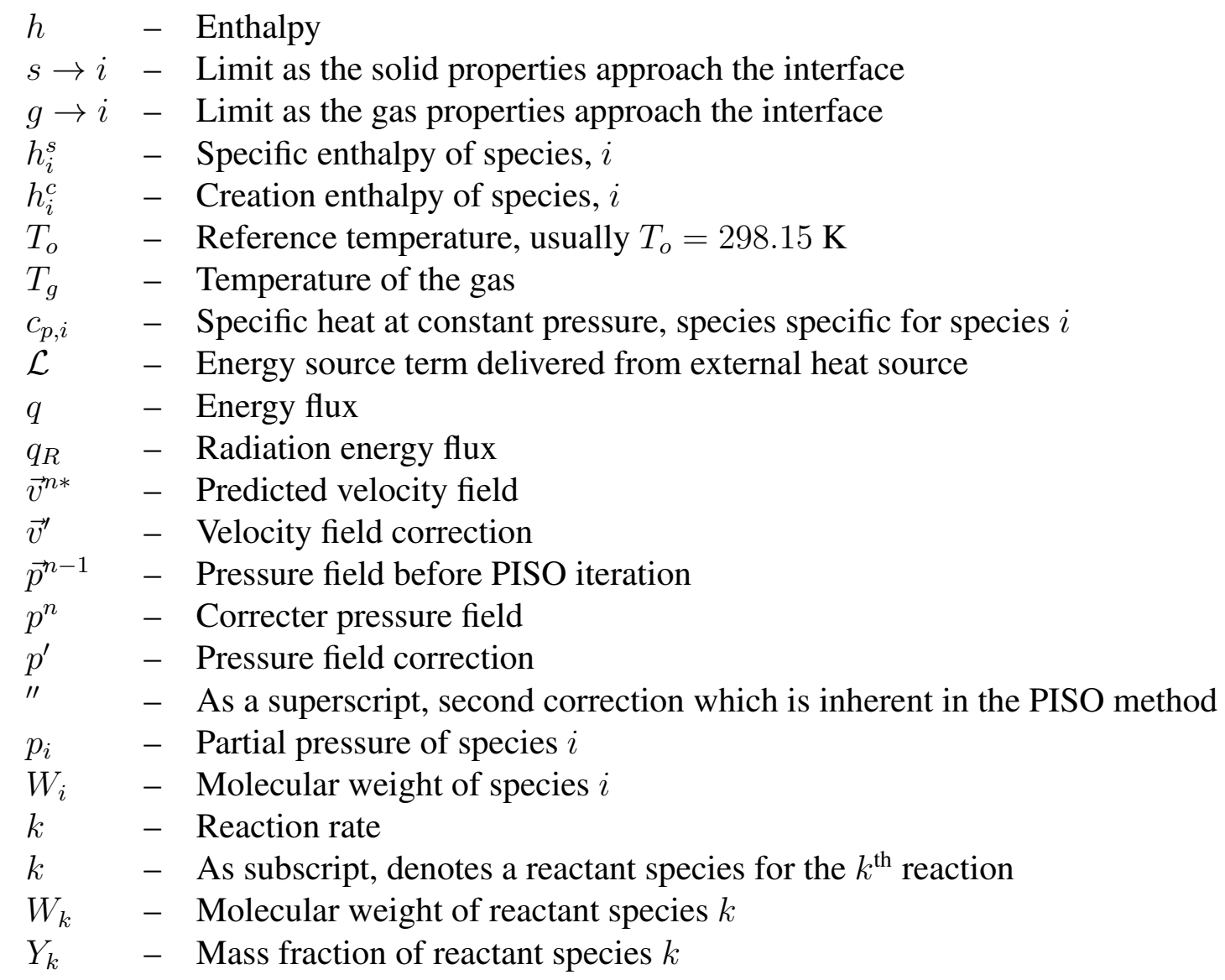

\section{Chapter 3}

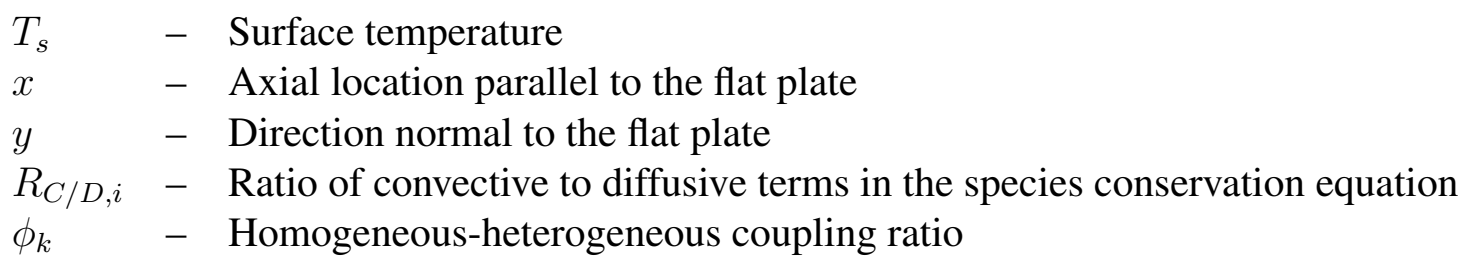




\section{Chapter 4}

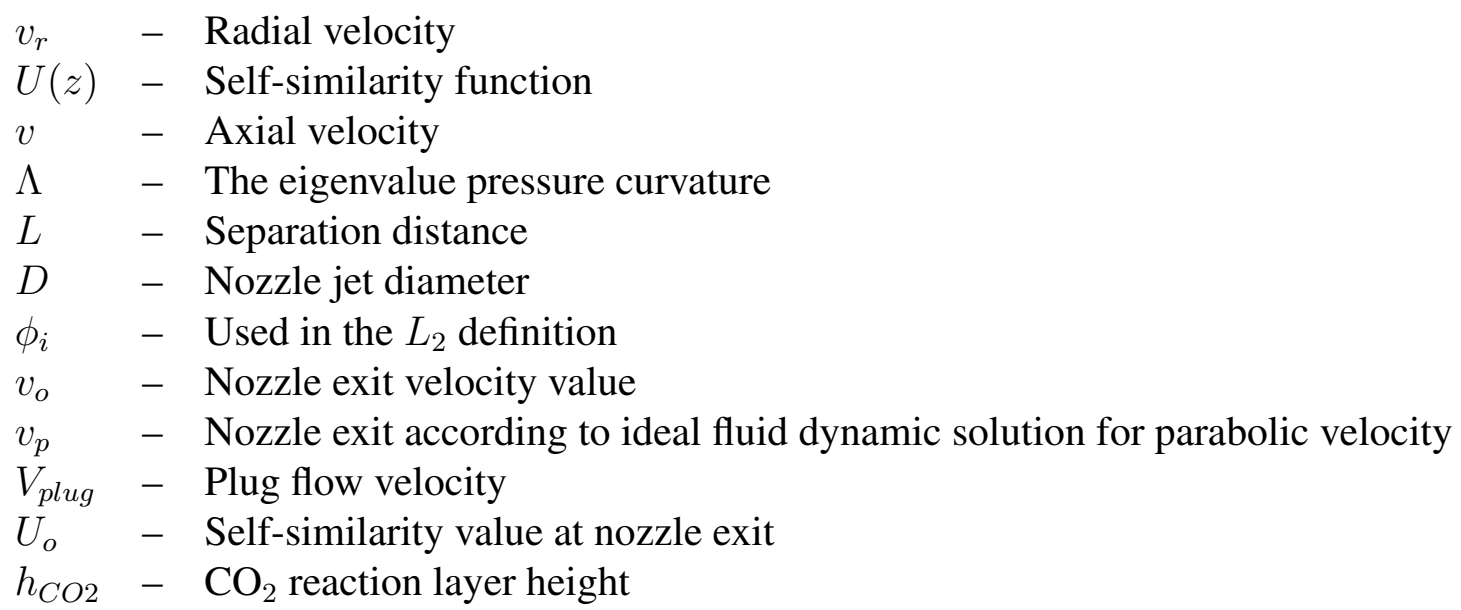




\section{Chapter 1}

\section{Introduction}

\subsection{Background}

The coupling of homogeneous (gas phase) and heterogeneous (gas phase) reactions is found in various phenomena, from the burning of wood in a fireplace to the ablating heat shield of the space shuttle flying at hypersonic speeds. Heterogeneous reactions consume gas phase species at a heated surface, which results in products that diffuse and convect into the gaseous domain. In most scenarios, the extreme heating, coupled with the presence of an oxidizer, causes additional reactions in the gas phase, which couples the heterogeneous and homogeneous reactions. These reacting scenarios exist at the core of many fields of engineering, from catalytic converters to coal bed combustion. Despite their importance, the engineering community is missing detailed analysis and methods to model these coupled homogeneous and heterogeneous reactions, especially when the fluid dynamics of the neighboring gas are complicated by multidimensional effects.

The present work was inspired by the Air Force Institute of Technology's (AFIT) interest in one such example of coupled homogeneous and heterogeneous reactions: the destruction of an enemy missile by laser induced ablation. Airborne-lasers are a well known option for the interception and destruction of ballistic missile threats. This defense tech- 
nology allows for the dynamic control of optical defense systems, giving it advantages over a one-shot, one-chance interceptor missile. Despite this advantage, airborne laser defense systems have a number of uncertainties. In particular, the time it takes for the heating by the laser to compromise the missile is dependent on a number of factors, missile material, laser effluence, angle of incidence, etc., which causes an inherent uncertainty that must be investigated [1]. In an effort to further understand these dependencies, AFIT and the University of Virginia are developing both experimental and computational methods that will be used to better understand and investigate coupled homogeneous and heterogeneous reactions. This work focuses on the development of numerical methods that were used to model the multidimensional coupling of homogeneous and heterogeneous reacting fluid dynamics. The methods were then applied to several fluid dynamic configurations, and detailed analysis is reported in this work.

The initial efforts of this collaborative research focus on reacting carbon surfaces. Missile surfaces are not made of carbon; however, the pre-existing research of coupled homogeneous and heterogeneous reactions of carbon surfaces serves as a foundation for the initial stages of this project. The research will then move towards different materials that are more representative of a missile surface. The framework of this research can be applied to any number of carbon surface reaction areas of interest. Additionally, the methods developed in this work are adaptable and can examine coupled homogeneous and heterogeneous reactions involving non-carbon surfaces, without modification to the core numerical model. This will become apparent in Chapter 2, where the derivation and modeling of reactions at an interface are derived for any heterogeneous reacting surface. and impinging stagnation point flow (stagnation flow)

This work concentrated on two multidimensional fluid dynamic configurations. The first of these configurations is the parallel flow over a reacting boundary layer (flat plate). A brief introduction for this configuration is presented in Section 1.3.1, and the in-depth analysis of the homogeneous and heterogeneous coupling is presented in Chapter 3. The 
second of these flow configurations is the axisymmetric stagnation point flow impinging on a reacting carbon plate, which is introduced in Section 1.3.2 and analyzed in detail in Chapter 4 . In addition to these configurations, Chapter 5 covers advanced fluid dynamic and surface interface conditions.

\subsection{Carbon Oxidation}

\subsubsection{History and Relevance}

Solid carbon oxidation, particularly coal combustion, has provided energy for over 500 years of human history [2]. Coal has shaped not only cultures but also our world climate. In the United States, coal is the third largest primary energy source, accounting for 18 percent of all energy consumed in 2013, with carbon dioxide emissions from coal combustion representing 24.5 percent of total U.S. greenhouse gas emissions [3]. Coal is such a powerful and integral resource that famous American writer, Ralph Waldo Emerson, highlighted its importance in his book, The Conduct of Life :

Watt and Stephenson whispered in the ear of mankind their secret, that half an ounce of coal will draw two tons a mile, and coal carries coal, by rail and by boat, to make Canada as warm as Calcutta; and with its comfort brings industrial power. [4]

The power of harnessing carbon oxidation has been known for quite some time, but the understanding of the complex physics involved in its burning still holds plenty of mystery. This research adds to the understanding of such an interesting physical process.

The work presented in this dissertation relied heavily on past existing models of carbon surface oxidation in order to model a carbon surface reacting in air. Relevant investigations date back to the late 1950's, where researchers focused on understanding char and soot oxidation [5-8]. These studies resulted in heterogeneous reaction models and dis- 
tinct carbon oxidation regimes were identified. At the same time, parallel investigations of carbon surface reactions focused on obtaining analytical solutions for the ablation and sublimation of thermal protection systems during NASA's Mercury, Gemini, and Apollo programs $[9,10]$. In the years since, interest in predicting reacting carbon surfaces has grown and resulted in a variety of numerical models including, but not limited to, graphite rocket nozzles [11-14], carbon particle burning [15], hypersonic re-entry [16], and packed coal bed combustion [17-20]

\subsubsection{The Coupling of Carbon Surfaces Reacting in Air}

At the core of these reacting carbon surface applications are the heterogeneous reactions that are inherently coupled with the neighboring gas phase. Figure 1.1 illustrates gaseous species and their coupled interaction at a reacting carbon surface. The reactions that occur between air and heated solid carbon, $\mathrm{C}_{s}$, at high surface temperatures ( greater than 1500 $\mathrm{K})$ produces mainly carbon monoxide, $\mathrm{CO}$. The conversion of solid carbon to $\mathrm{CO}$ causes a gaseous expansion and a convective velocity. This convection, combined with species diffusion, transports the carbon monoxide into the neighboring gaseous domain. The carbon monoxide then mixes and reacts with oxygen, $\mathrm{O}_{2}$, to create carbon dioxide, $\mathrm{CO}_{2}$, which then diffuses back to the surface and reacts, forming additional carbon monoxide, and thereby couples the heterogeneous and homogeneous reactions [21-23]. 


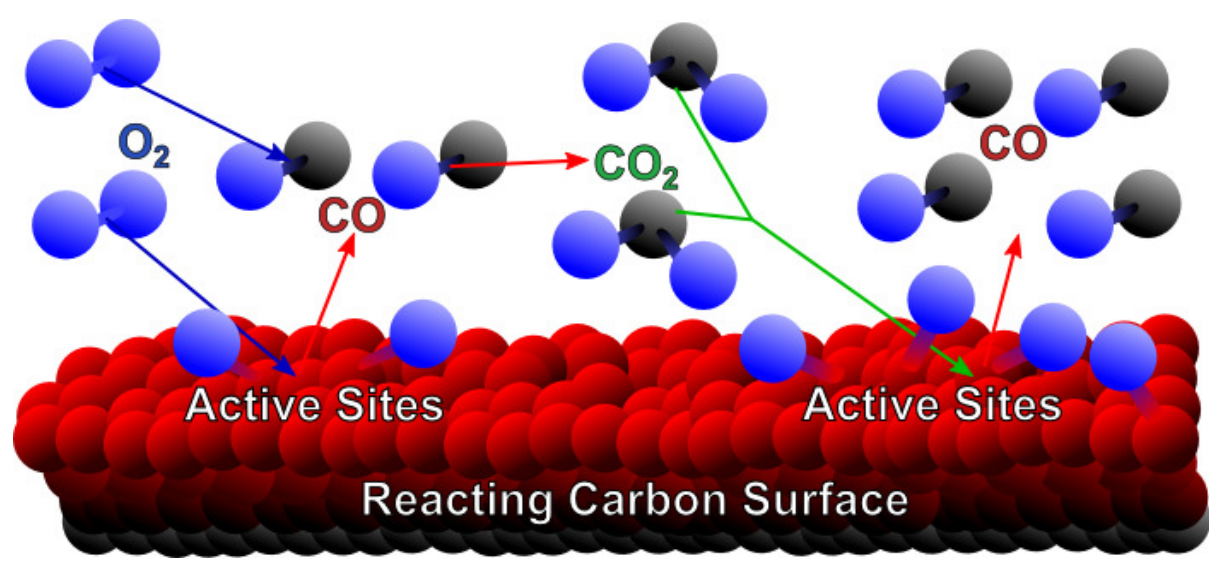

Figure 1.1: Reacting carbon surface with $\mathrm{C}_{s}+\mathrm{O}_{2} \rightarrow \mathrm{CO}, \mathrm{CO}+\mathrm{O}_{2} \rightarrow \mathrm{CO}_{2}$, and $\mathrm{C}_{s}+\mathrm{CO}_{2} \rightarrow 2 \mathrm{CO}$.

Semi-global surface reactions are used to model not only these reactants, $\mathrm{O}_{2}$ and $\mathrm{CO}_{2}$, but also $\mathrm{H}_{2} \mathrm{O}$, and radicals species, $\mathrm{OH}$ and $\mathrm{O}$. The available models differ slightly, but the majority of them contain reaction pathways that produce $\mathrm{CO}$ and traces of $\mathrm{H}$ and $\mathrm{H}_{2}$. The models used in this work come from experimental data generated by nonporous graphite experiments. The experiments resulted in a heterogeneous reaction model that was collected in Ref. [24]. To explore carbon porosity, this work used another model that tuned the reactions collected in Ref. [24] to include carbon surface porosity of about 25\% [25]. These heterogeneous models contain reaction rate information and depend on the surface's thermodynamic properties and the reactant species concentrations.

\subsubsection{Past Reacting Carbon Surface Modeling Work}

Extensive work has been done by Makino and his associates over the past 30 years, where a stagnation flow configuration (covered in Section 1.3.2) was utilized to validate surface reaction model parameters (ie. collision frequency and activation energy) by measuring carbon mass loss rates. The results of such studies can be seen in reviews $[26,27]$ and recent works $[20,28,29]$. These studies show that surface temperatures and fluid dynamics affect reacting layers, which is supported by other works, such as Refs. [17-19]. Additionally, several groups have used carbon surface reaction models in graphite nozzle ablation 
studies [11-14]. The results of these studies extract the controlling factors in graphite nozzle ablation and show the multidimensionality of these reacting layers. However, gaseous $\mathrm{O}_{2}$ and oxygen atoms, $\mathrm{O}$, were disregarded in their work as they are assumed neglible in nozzle ablation, making these graphite nozzle results inapplicable to carbon surfaces reacting in air.

These works either reduced the models to ignore multidimensionality or neglected necessary components to adequately assess the coupling that exists when carbon surfaces react with air. To date, Shulze et al. [30] is the only group that investigated the multidimensionality of the reacting layers of carbon surfaces in an air environment. Their group studied flow over a moving reacting carbon sphere with diameter $2 \mathrm{~mm}$ and concluded that flow parameters and surface temperatures affect the reacting layers over the sphere but did little to address what these results mean for the broad spectrum of reacting carbon surfaces. The objective of this research is to better understand the effects that fluid dynamic configurations have on coupled heterogeneous and homogeneous reactions, specifically when the flow is multidimensional. The full list of objectives are presented in Section 1.5

\subsection{Fluid Dynamic Configurations}

Figure 1.2 illustrates subsonic, steady-state, laminar flow over a reacting solid body. Two fundamental flow configurations are formed when a blunt body is subjected to free stream flow: flow over a flat surface and stagnation flow on a surface. These two flow configurations are easily created in laboratory experiments and have precedence in various analytical research initiatives, which is covered in Sections 1.3.1 and 1.3.2. The coupling of the reacting surface and gas phase creates complications that cannot be captured through previously developed analytic descriptions. It is therefore import to simulate both flow configurations through numerical methods that include multidimensionality in order to solve for the com-

plex physics in the viscous regions that host momentum, thermal, and species boundary 
layers.

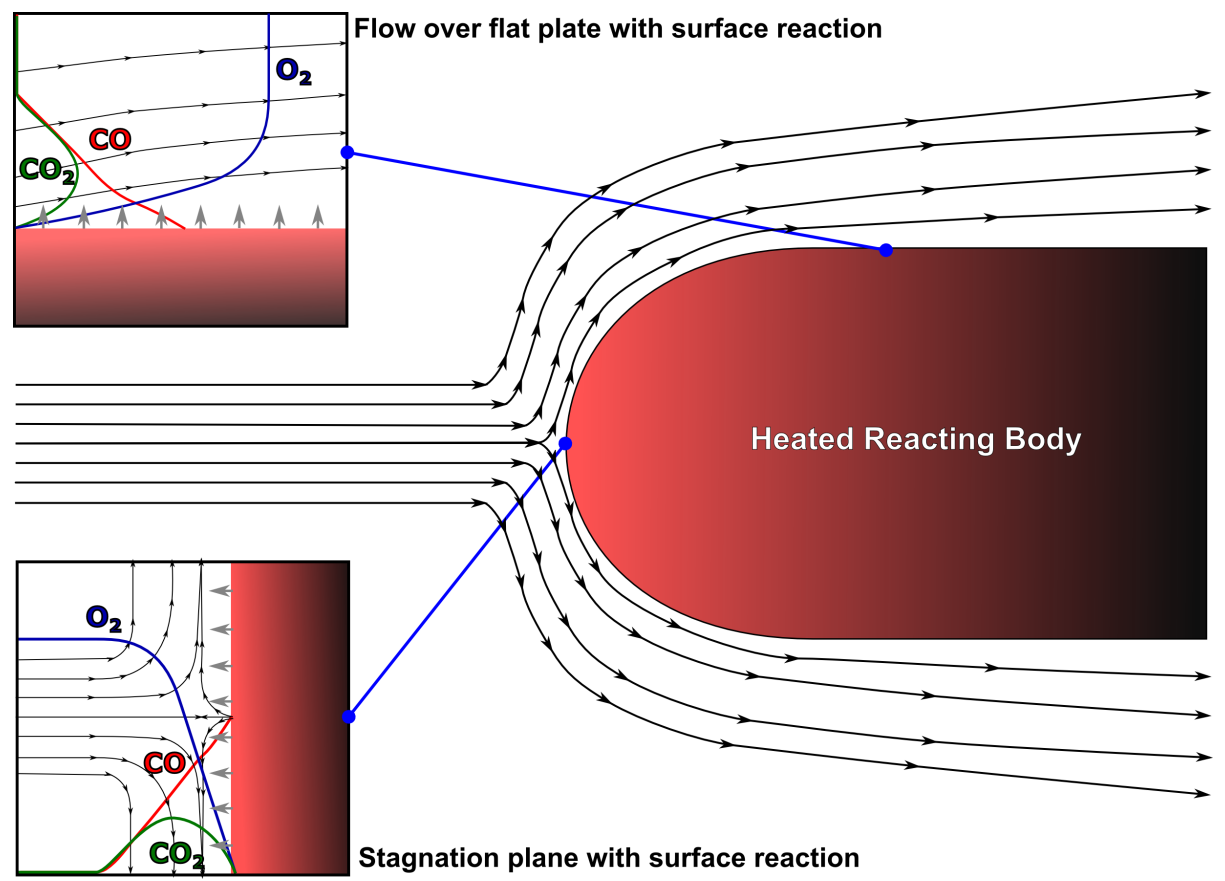

Figure 1.2: Reacting blunt carbon body subjected to stagnant and flat plate flow

\subsubsection{Parallel Flow of Air Over a Reacting Flat Plate}

When a fluid flows tangentially to a flat plate, a thin viscous region exists where the axial velocity, $u$, transitions from $u=0$ to the free-stream quantity, $u=U_{\infty}$. This flow configuration was first addressed in 1904, when Ludwig Prandtl presented his revolutionary paper on inviscid fluid flow and began the field of momentum boundary layer theory. In the years since, momentum transport theory applications have expanded to a variety of research topics.

Momentum boundary layer theory describes the transition of the axial velocity to the free stream, parallel flow, $u \rightarrow U_{\infty}$. This transition region grows in height along the downstream direction of the plate and is inversely proportional to the local Reynolds number, $\delta \sim R_{x}^{-1 / 2}=\left(\frac{x U_{\infty}}{\nu}\right)^{-1 / 2}$. It is important to note that several historical attempts to describe the flat plate laminar velocity profile, $u=U(y)$, were made using various assumptions, the most famous are the Blasius and Falkner-Skan flows. These descriptions 
work extremely well for strict fluid dynamic conditions imposed ${ }^{1}$.

Despite the availability of these valid approximations for momentum boundary layers, reacting surfaces add complications that cannot be easily accounted for. When a reacting flat surface is subjected to parallel flow, the heterogeneous reactions coupled with thermal boundary layers prevent the reductions necessary to form a self-similar model. Furthermore, for reacting carbon surfaces, the heterogeneous reactions cause mass addition to the flow and results in a blowing velocity, $v_{n}$, from the surface. Such a complex configuration is illustrated in Figure 1.3, which shows a reacting carbon surface with species boundary layers, fluid flow stream lines, and blowing velocity vectors.

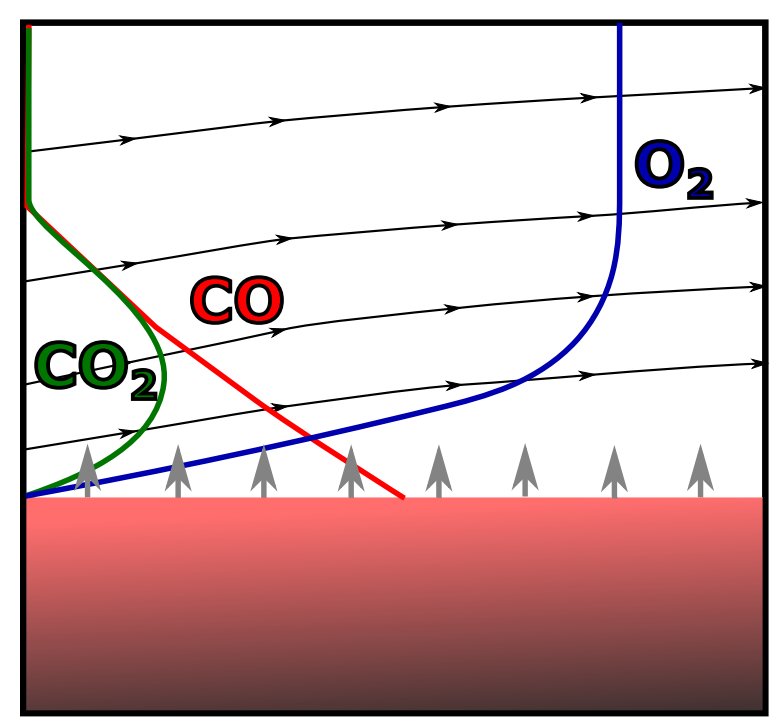

Figure 1.3: Illustration of reacting flow over flat plate. The red material is the reacting carbon surface, lines with arrows are fluid streamlines, and vectors along the surface represent mass added by blowing velocity, $v_{n}$.

Recent work on flow control surfaces has proposed various models that incorporate surface blowing and suction. A review of this work can be found in Ref. [32], however, these attempts do not include surface or gas phase reactions.

The velocity magnitude of the fluid near the flat plate is considerably less than that of the free stream region. This means that the fluid residence time ${ }^{2}, \tau_{r e s}$, is inversely propor-

\footnotetext{
${ }^{1}$ Further details on Reynoylds number, Blasius flow, and Falkner-Skan flows as well as their applicability can be found in Ref. [31]

${ }^{2}$ Residence time is defined loosely as the average amount of time that a particle along a fluid streamline
} 
tional to distance from the flat plate, $\tau_{\text {res }} \sim y^{-1}$. This longer residence time, along with the large temperatures within the thermal boundary layer, causes the gaseous reactions close to the plate and continue as the fluid is carried downstream. The coupling of heterogeneous and homogeneous reactions create unique reaction profiles that change according to the freestream velocity (which directly affects $\tau_{r e s}$ ), surface temperature, species concentrations, and surface porosity. Furthermore, higher reactivity near the leading edge and depletion of oxygen complicates the boundary layer structure.

The combined effects of the thermal boundary layer, surface and gaseous reactions, and blowing velocity adds a considerable amount of complexity to the flat plate flow configuration. The gaseous reactions that happen in the axail direction were not expected to be selfsimilar and hence analytical reduced solutions were not considered. To generate solutions, the finite volume method, described in Chapter 2, was used to simulate two-dimensional parallel flow over a reacting flat plate.

\subsubsection{Stagnation Point Flow Over a Reacting Carbon Surface}

The fluid flow normal to the surface, or stagnation flow, is opposed by the carbon surface's blowing velocity. This is much like the counterflow, or opposed jet, reacting flow configuration which has been used extensively to understand the interaction of flow residence time and finite rate kinetics [33]. Figure 1.4 illustrates this counterflow flame configuration.

spends in a particular system $\tau_{r e s}=\frac{\text { Characteristic Distance }}{\text { Characteristic Velocity }}$. For example, a car engine injects fuel and air into a piston and combusts these gases to drive the piston. The remaining gas is removed as exhaust. The time the fuel and air have to combust is viewed as the residence time of that system. 


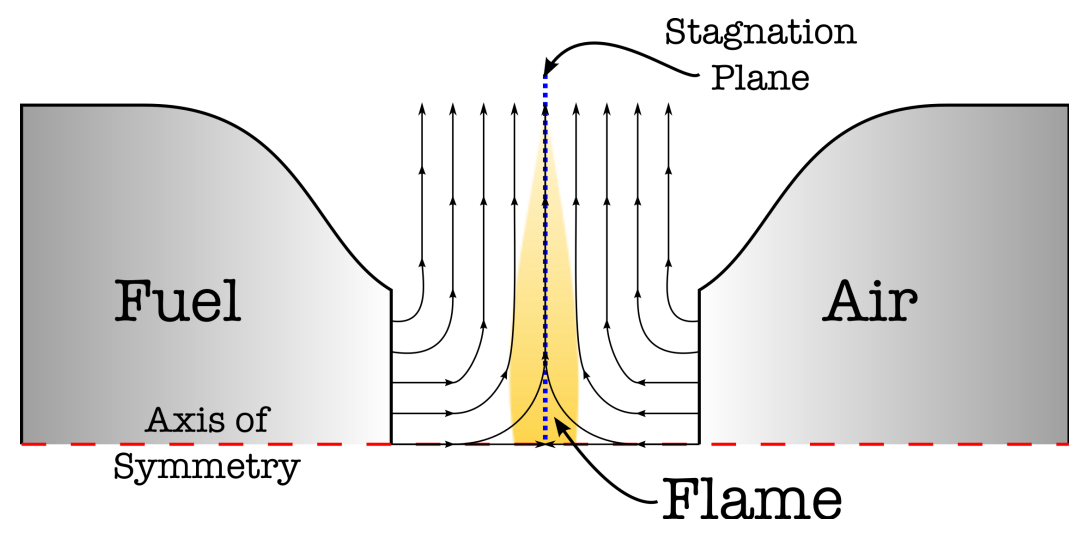

Figure 1.4: Illustration of an axisymmetric counterflow flame with convergent nozzles.

In this configuration, flow is injected into a mixing region from two opposed nozzles, one containing the oxidizer and the other containing the fuel. The two streams meet at a stagnation point and diverge radially outwards. The location of this axisymmetric divergence is known as the stagnation point. In the vicinity of this point, chemical reactions are sustained by either heating one of the nozzle flows or igniting the mixing layer from an external source. The flame is held at steady-state due to the constant flow of the fuel and oxidizer into the reacting region while keeping the opposed momenta balanced.

As the opposed jet velocities are increased, a limit is reached where the flame temperature decreases until reactions cease to exist. This is known as the counterflow flame extinction limit. As the jet velocities increase, so does the velocity gradient or strain rate near the stagnation plane. This gradient is inversely proportional to the fluid dynamic residence time, $\tau_{\text {res }}$. At high velocity gradients the fluid dynamic residence time approaches that of the chemical reaction time scales. Within this transitional region, a flame reaches extinction because it can no longer sustain chemical reactions within the fluid dynamic time constraints.

In the reacting stagnation flow configuration considered in this dissertation, the freestream air is flowing normal to the reacting surface, as seen in Figure 1.5. 


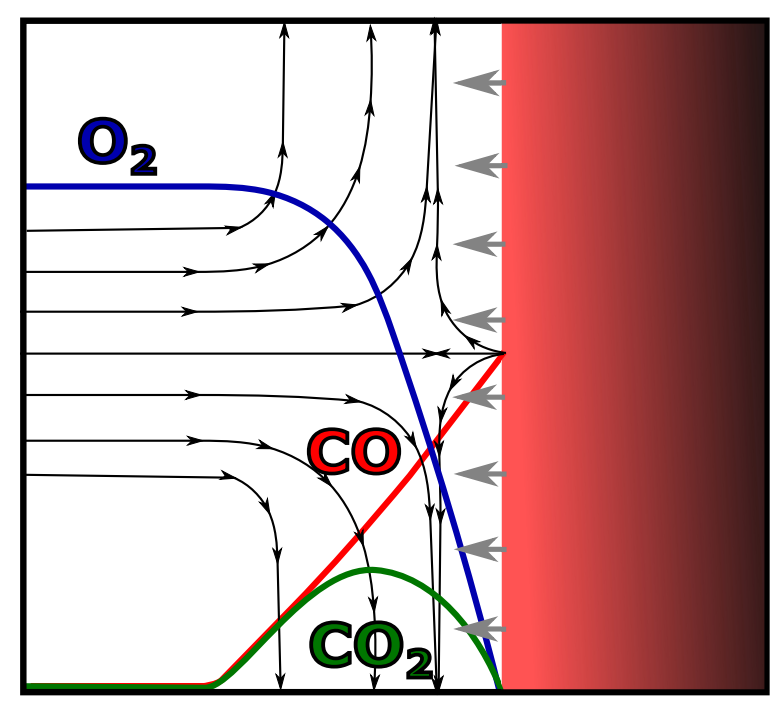

Figure 1.5: Illustration of reacting stagnation flow. The red material is the reacting carbon surface, lines with arrows are fluid streamlines, and vectors along the surface represent mass addition

As the surface reacts, carbon monoxide is ejected into the free stream air and creates a stagnation plane. The opposing streams diverge in the radial direction forming a fluid dynamic scenario similar to the canonical counterflow flame. Therefore, at high velocities, the gaseous reactions that form $\mathrm{CO}_{2}$ reach an extinction limit, whether or not that extinction is sudden or gradual with increase in imposed velocity will be answered in this work.

Currently, counterflow flames are modeled using the historical quasi one-dimensional formulation that assume self-similarity for radial-direction velocities [34-36]. These formulations can easily account for surface reactions at a wall; however, the quasi onedimensional assumptions restrict the possibility to capture multidimensional effects that differ from the assumed self-similarity and imposed boundary conditions. A full review of these issues is found in Section 4.2, and the challenges of using the quasi one-dimensional formulation in this work is addressed. 


\subsection{Supporting Experimental Efforts}

The flat plate and stagnation flow configurations will be utilized in the parallel experimental efforts. Researchers at AFIT have already started experiments that measured plume species concentrations from heated surface reactions using imaging Fourier transform spectroscopy (IFTS) [37]. Specifically, experiments are performed with a hyperspectral imaging camera with line-of-sight data. The images collected are combined to form a three-dimensional, $(x, y, \lambda)$, hyperspectral data space for processing and analysis. The data set gives wave lengeth, $\lambda$, as a function of $x$ and $y$ locations. In these experiments, data is acquired with a spectral resolution of $2 \mathrm{~cm}^{-1}$ and spatial resolution as high as $0.52 \mathrm{~mm}^{2}$ per pixel in a two dimensional plane, with framing rates up to $2.5 \mathrm{~Hz}$. A fast Fourier transform (FFT) of each pixel's interferogram produces a raw spectrum, which is then calibrated with two internal wide-area black body sources at known temperatures. Using established radiometric calibration procedures the spectrally dependent gain and background for each pixel are determined [38] and species concentrations are extracted. This IFTS alalysis of the spectra at each pixel gives two-dimensional reacting structures of $\mathrm{H}_{2} \mathrm{O}, \mathrm{CO}, \mathrm{CO}_{2}$ concentrations over the reacting carbon surface.

Difficulties due to bouyancy effects in the flat plate experiments made one-to-one comparison between experiments and computations impossible. Extra problems arose in controlling air flow over the surface, and, because of other factors, the flat plate configuration did not generatre results that led to one-to-one comparison. In an effort to better control flow conditions over the reacting carbon surface, an experimental stagnation flow configuration like the one shown in Figure 1.6 was proposed and are being conducted at AFIT. The main difference between this experimental configuration and typical counterflow experiments is that the impinging flow is delivered by a tube-jet and not a convergent nozzle with a top-hat profile. 


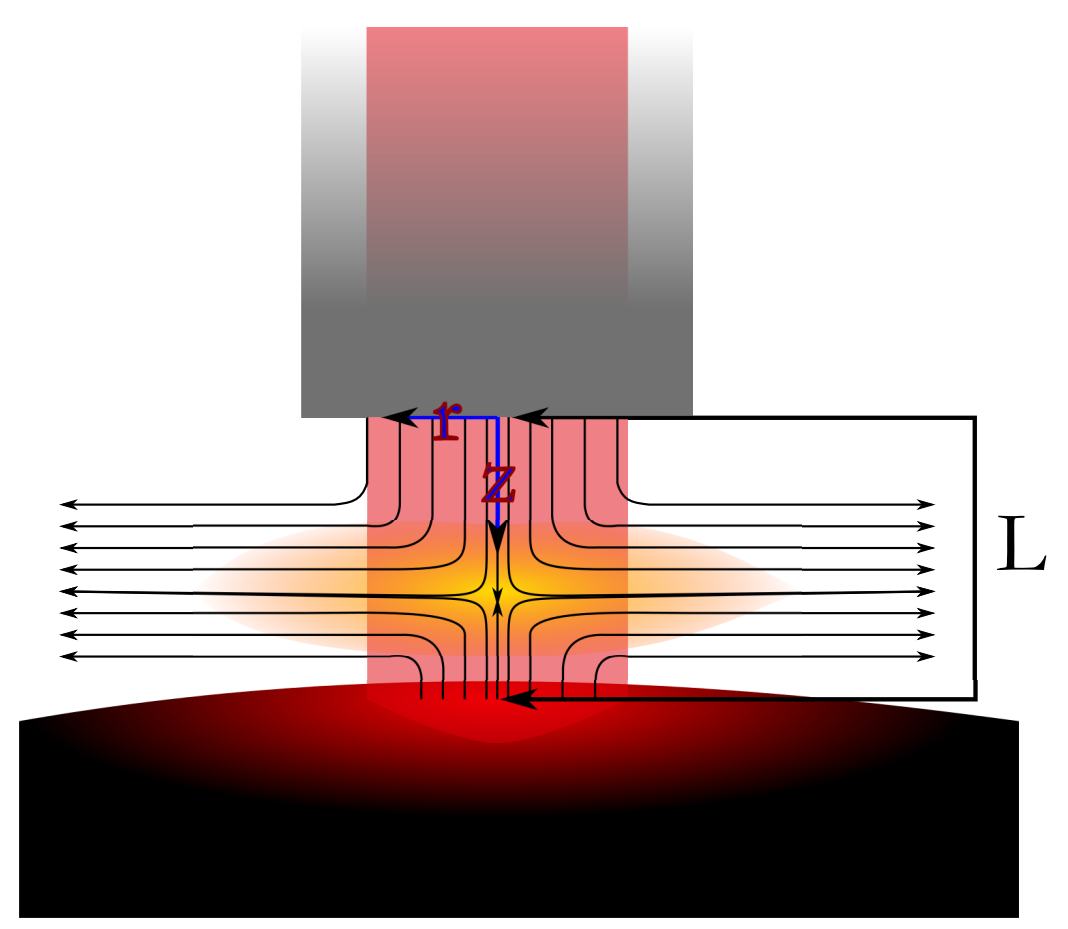

Figure 1.6: Illustration of reacting stagnation flow experimental configuration . Here, the black curved material is the heated carbon, lines with arrows are fluid stream lines, $r$ and $z$ are coordinates, and $\mathrm{L}$ is the separation distance. Also shown is an illustration of the laser concentrically shot through the tube-jet (shown in gray).

In this configuration a continuous laser beam capable of delivering 10-1000 W/ $\mathrm{cm}^{2}$ energy flux rates will be shot concentrically through the tube-jet. While the IFTS will be used to process hyperspectral imaging data, the surface recession rates of the carbon will also be measured through the use of a video camera. In order to provide a better line-of-sight for video measurements of the surface recession rate, the carbon surface will be slightly curved. The response of reacting structures and mass loss rate to tube-jet velocity, carbon surface model, and surface temperature will create an excellent one-to-one comparison framework, which will then be used to validate carbon surface reaction models in multidimensional configurations. Due to the issues in flat plate experimental data and the delay of stagnation flow experiments the collaborative component of this work highlights the key trends that are expected in the upcoming experiments at AFIT. 


\subsection{Objectives}

Listed here are the three objectives of this research, each concentrating on analyzing different consequences and aspects of the two flow configurations coupled with homogeneous and heterogeneous reactions.

\subsubsection{Analyze the applicability of heterogeneous kinetic models in complex geometries}

Heterogeneous reaction models were developed using very simple flow geometries and it has yet to be determined whether they can be properly used in a more complex flow simulations, despite their use in previously mentioned research. The choice of heterogeneous reaction model and dependency on fluid flow configurations were analyzed and are discussed in detail in Chapters 3, 4, and 5.

\subsubsection{Assess extinction and blow-off limits of gas phase reactions}

Both flow configurations have velocity conditions that directly affect carbon surface reaction layers. An increase in free stream velocity in the flat plate flow configuration caused the reaction layers to be swept downstream of the reacting plate. Although it was expected that the $\mathrm{CO}_{2}$ reaction would be fully swept off the reacting region, referred to as blow-off, no such limit was reached. Instead, $\mathrm{CO}_{2}$ values decreased with an increase in velocity and the affect of flow velocity on the terms of species conservation equation is examined in this work. The effect of the fluid dynamics in this flow configuration is analyzed in detail in Chapter 3.

The stagnation flow configuration was expected to reach an extinction limit where the reactions that creates $\mathrm{CO}_{2}$ would cease to exist due to fluid flow velocity. As is discussed in Chapter 4, the extinction limit is approached as flow velocity approached $\tilde{1} 00 \mathrm{~m} / \mathrm{s}$. This speed is too high for experimental considerations. However, another limit was found in- 
volving $\mathrm{CO}_{2}$ flame attachment/detachment that depends on in-flow velocity. Interesting reaction layers that can be explored experimentally and the physics involving flame detachment are discussed in detail in Chapter 4.

\subsubsection{Identify optimal experimental configurations for experimental validation efforts}

Experimentalists at AFIT are currently exploring the reaction layers caused by laser induced carbon heating in order to better understand the physics involved in high energy laser defense technologies. Experiments use a hyper-spectral imaging camera, which has the ability to detect and quantify the important chemical species $\left(\mathrm{CO}, \mathrm{CO}_{2}, \mathrm{NO}_{x}, \mathrm{H}_{2}\right.$, etc), with the intent of further understanding the heterogeneous surface reactions in complex fluid flow geometries. The time consuming nature of experiments is where the numerical simulations of this work are advantageous. Key trends worthy of experimental exploration were revealed in this work and are discussed throughout Chapters 3, 4, and 5. 


\section{Chapter 2}

\section{Formulation and Numerical Modeling}

I don't believe in the idea that there are a few peculiar people capable of understanding math, and the rest of the world is normal. Math is a human discovery, and it's no more complicated than humans can understand... What we've been able to work out about nature may look abstract and threatening to someone who hasn't studied it, but it was fools who did it, and in the next generation, all the fools will understand it.

-Richard P. Feynman, The Pleasure of Finding Things Out pg.194 


\subsection{The Reacting Navier Stokes Equations and Model For- mulation}

The OpenFOAM computational package was used to model the following reacting NavierStokes equations,

$$
\begin{aligned}
\frac{\partial \rho}{\partial t}+\nabla \cdot \rho \vec{v} & =0 \\
\frac{\partial \rho \vec{v}}{\partial t}+\nabla \cdot(\rho \vec{v} \vec{v}) & =-\nabla p+\nabla \cdot \mathbb{T}, \\
\frac{\partial \rho h^{s}}{\partial t}+\nabla \cdot \rho \vec{v} h^{s}-\nabla \cdot \rho \alpha \nabla h^{s}+\nabla \cdot \sum_{i=1}^{N} \rho h_{i}^{s} \vec{V}_{i} & =-\sum_{i=1}^{N} h_{i}^{c} w_{i}, \\
\frac{\partial \rho Y_{i}}{\partial t}+\nabla \cdot \rho \vec{v} Y_{i}-\nabla \cdot \rho D_{i} \nabla Y_{i} & =w_{i},
\end{aligned}
$$

where $\vec{v}$ is the velocity vector, $p$ is pressure, $\rho$ is density, $D_{i}$ is mixture-averaged diffusion coefficient of species $i, \mathbb{T}$ is the deviatoric stress tensor, $\alpha$ is the thermal diffusivity of the mixture, $h_{i}^{c}$ and $h_{i}^{s}$ are the enthalpy of creation and sensible enthalpy of species $i$ respectively, $\vec{V}_{i}$ is the mixture average diffusion velocity of species $i, Y_{i}$ is mass fraction of species $i$, and $w_{i}$ is the mass production rate of species $i$. The conservation equations are solved in a segregated manner using second-order accurate total variation diminishing (TVD) Van-Leer schemes. The finite volume equations are integrated in time using the first-order implicit, Eulerian method. Each equation is solved by iterating until the $\mathrm{L}_{2}$-norm of the residual to all equations falls below $10^{-9}$. Simulations are run for a specific number of time integrations until steady state is reached and confirmed by monitoring solution residuals.

Velocity and pressure are coupled via the $\nabla p$ term in Equation 2.2. A typical compressible flow solver would treat $p$ as both the hydrodynamic and thermodynamic pressure and solve for it using the equation of state. This approach causes acoustic waves to exist through the continuity equation and pressure coupling (density changes cause pressure changes via 
the equation of state). These acoustic waves are ideal for high-speed scenarios, as they can capture shock-wave phenomena. However, these acoustic waves are computationally costly for low-speed cases as they restrict time steps to be on the order of acoustic time scales and typically cause unstable, oscillatory solutions. To avoid these pressure waves, the pressure in the equation of state is assumed a constant value. This pressure in the equation of state is the thermodynamic pressure, $p_{o}$.

The velocity magnitudes considered in this work are, at most, $50 \mathrm{~m} / \mathrm{s}$. This gives dynamic pressure changes, $\frac{1}{2} \rho \vec{v} \cdot \vec{v}$, on the order of one percent of an atmospheric pressure. Therefore, it is an appropriate assumption that the equation of state only provide closure between temperature and density, by assuming thermodynamic pressure as constant,

$$
\rho=\frac{p_{0} \bar{M}}{R T}
$$

Here, $p_{0}$ is the thermodynamic pressure (typically atmospheric unless the low-speed flow is within a pressurized chamber), $\mathrm{R}$ is the universal gas constant, and $\bar{M}$ is the mixture averaged molecular weight of the gas. The constant thermodynamic pressure decouples the hydrodynamic pressure ${ }^{1}$ from density, which results in the removal of unwanted pressure waves associated with small density transients. This assumption is advantageous; however, an explicit equation for pressure is required. By taking the divergence of the momentum equation an explicit pressure equation is obtained,

$$
\begin{array}{r}
\nabla \cdot\left(\frac{\partial \rho \vec{v}}{\partial t}+\nabla \cdot(\rho \vec{v} \vec{v})\right)=\nabla \cdot(-\nabla p+\nabla \cdot \mathbb{T}) \\
\nabla^{2} p=\nabla \cdot\left(-\frac{\partial \rho \vec{v}}{\partial t}-\nabla \cdot(\rho \vec{v} \vec{v})+\mathbb{T}\right)
\end{array}
$$

Equation 2.6 is the pressure Poisson equation (PPE). To calculate pressure, the work presented here used the pressure implicit splitting of operators (PISO) method [39], which iterates between the solved velocity field and a guessed pressure distribution using a form of the

\footnotetext{
${ }^{1}$ The driving pressure found in the conservation equation. It can be seen as a potential for driving flow.
} 
PPE until a specified numerical tolerance is met. This pressure solving technique is computationally efficient and has precedence in the combustion community (see Refs. [18,40,41]). The PISO method is presented in detail in Section 2.4.3. Additionally, Ref. [42] is an excellent reference for more information on this decoupled approach for low-Mach number, steady, reacting flows.

The steps used in the developed solver which include equations 2.1-2.4 are as follows:

1. Solve the continuity equation, $\frac{\partial \rho}{\partial t}+\nabla \cdot \rho \vec{v}=0$, for density fluxes.

2. Use external subroutine to calculate viscocity, $\mu$

3. Solve the momentum equation, $\frac{\partial \rho \vec{v}}{\partial t}+\nabla \cdot(\rho \vec{v} \vec{v})=-\nabla p+\nabla \cdot \mathbb{T}$, for velocities based on constant hydrodynamic pressure gradient, $\nabla p$.

4. Use an external subroutine to calculate chemical source terms, $w_{i}$, and diffusion coefficients, $D_{i}$

5. Solve the species conservation equation for species $1 \rightarrow N-1$,

$\frac{\partial \rho Y_{i}}{\partial t}+\nabla \cdot \rho \vec{v} Y_{i}-\nabla \cdot \rho D_{i} \nabla Y_{i}=w_{i}$. For species $Y_{N}$, the relation $Y_{N}=1-\sum_{i=1}^{N-1} Y_{i}$ is used.

6. Use an external subroutine to calculate thermal diffusivity, $\alpha$.

7. Solve energy conservation equation,

$$
\frac{\partial \rho h^{s}}{\partial t}+\nabla \cdot \rho \vec{v} h^{s}-\nabla \cdot \rho \alpha \nabla h^{s}+\nabla \cdot \sum_{i=1}^{N} \rho h_{i}^{s} \vec{V}_{i}=-\sum_{i=1}^{N} h_{i}^{c} w_{i}
$$

8. Update density based on thermodynamic pressure, $p_{0}$ (assumed constant), $\rho=\frac{p_{0} \bar{M}}{R T}$

9. Solve the PPE, $\nabla^{2} p=\nabla \cdot\left(-\frac{\partial \rho \vec{v}}{\partial t}-\nabla \cdot(\rho \vec{v} \vec{v})+\mathbb{T}\right)$, to correct the pressure field based on these changes and update velocity using this correction (PISO method).

10. Repeat Steps 1-9 until the residuals of all equations fall below a specified tolerance of $10^{-9}$ 
As covered in Section 2.3, the OpenFOAM package provides an elegant approach to solving these equations. The main limitation, however, is that the solver lacks the ability to produce time accurate solutions. The reasons are two-fold. First, the equations are solved in a segregated manner and treat gradient terms, e.g. $\nabla p$, as explicit, source terms. This also requires that the stability conditions, i.e. Courant number, Co [43], be satisfied and removes the ability to solve the equations fully implicitly. Local compressible Courant numbers are determined by OpenFOAM, and the fluid dynamic time step is updated accordingly (these results used $\max (\mathrm{Co})<0.4$ ). Secondly, chemical reactions require that a fully coupled system resolve small time scales (sometimes on the order of $10^{-9} \mathrm{~s}$ ), which is not a computationally feasible approach. The solver used for this work completely decouples the chemical source term integrations. This approach is suitable for achieving steady-state solutions; however, this solver cannot achieve fully time-resolved solutions which is its major limitation.

\subsubsection{Gas Phase Chemistry and Transport Properties}

Equation 2.4 contains a chemical source term, $w_{i}$, that is calculated from a set of elementary reaction rate expressions as Step 4,

$$
w_{i}=\sum_{j=1}^{N_{R}} \nu_{j, i}\left(B_{j} T^{\alpha_{j}} \exp \left(-E_{j} / R T\right) \prod_{i=1}^{N} X_{i}^{\nu_{j, i}^{\prime}}-\frac{1}{K_{c, j}} B_{j} T^{\alpha_{j}} \exp \left(-E_{j} / R T\right) \prod_{i=1}^{N} X_{i}^{\nu_{j, i}^{\prime \prime}}\right)
$$

where $T$ is the gas temperature; $j$ refers to the specific reaction of $N_{R}$ reactions; $i$ refers to the species of interest of $N$ total species; $B_{j}, \alpha_{j}$ and $E_{j}$ are the reaction model coefficeints; $R$ is the universal gas constant; $X_{i}$ is the mole fraction of species $i$; $\nu^{\prime}$ and $\nu^{\prime \prime}$ are forward and backward stoichemetric coefficients, respectively; $K_{c, j}$ is the reaction rate constant determined from thermodynamic properties; and $\nu_{j, i}=\nu_{j, i}^{\prime \prime}-\nu_{j, i}^{\prime}$. The reaction model coefficeints used in this work were supplied by the Yetter et al. [44] model and are listed in Table 2.1. The chemical source term expression is integrated in time using OpenFOAM's 
ordinary differential equation solvers.

Table 2.1: The specific reaction-rate constants for the $\mathrm{CO} / \mathrm{H}_{2} \mathrm{O} / \mathrm{O}_{2}$ mechanism from Yetter et al. in the form $k_{j}=B_{j} T^{\alpha_{j}} \exp \left(-E_{j} / R T\right)$.

\begin{tabular}{|c|c|c|c|c|}
\hline Step & Reaction & $B_{j}$ & $\alpha_{j}$ & $E_{j}$ \\
\hline 1 & $\mathrm{H}+\mathrm{O}_{2} \rightleftharpoons \mathrm{OH}+\mathrm{O}$ & $1.91 \times 10^{14}$ & 0.0 & 16440 \\
\hline 2 & $H_{2}+O \rightleftharpoons O H+H$ & $5.13 \times 10^{4}$ & 2.67 & 6290 \\
\hline 3 & $\mathrm{H}_{2}+\mathrm{OH} \rightleftharpoons \mathrm{H}_{2} \mathrm{O}+\mathrm{H}$ & $2.14 \times 10^{8}$ & 1.51 & 3430 \\
\hline 4 & $\mathrm{OH}+\mathrm{OH} \rightleftharpoons \mathrm{O}+\mathrm{H}_{2} \mathrm{O}$ & $k=5.46 \times 10^{11}$ & $\exp (.00149 \times T)$ & \\
\hline 5 & $H_{2}+M \rightleftharpoons H+H+M^{a}$ & $4.57 \times 10^{19}$ & -1.4 & 104380 \\
\hline 6 & $O+O+M \rightleftharpoons O_{2}+M^{a}$ & $6.17 \times 10^{15}$ & -0.5 & 0 \\
\hline 7 & $O+H+M \rightleftharpoons O H+M^{a}$ & $4.68 \times 10^{18}$ & -1.0 & 0 \\
\hline 8 & $H+O H+M \rightleftharpoons H_{2} O+M^{a}$ & $2.24 \times 10^{22}$ & -2.0 & 0 \\
\hline 9 & $H+\mathrm{O}_{2}+M \rightleftharpoons \mathrm{HO}_{2}+M^{a}$ & $6.76 \times 10^{19}$ & -1.42 & 0 \\
\hline 10 & $\mathrm{HO}_{2}+\mathrm{H} \rightleftharpoons \mathrm{H}_{2}+\mathrm{O}_{2}$ & $6.61 \times 10^{13}$ & 0.0 & 2130 \\
\hline 11 & $\mathrm{HO}_{2}+\mathrm{H} \rightleftharpoons \mathrm{OH}+\mathrm{OH}$ & $1.70 \times 10^{14}$ & 0.0 & 870 \\
\hline 12 & $\mathrm{HO}_{2}+\mathrm{O} \rightleftharpoons \mathrm{OH}+\mathrm{O}_{2}$ & $1.74 \times 10^{13}$ & 0.0 & -400 \\
\hline 13 & $\mathrm{HO}_{2}+\mathrm{OH} \rightleftharpoons \mathrm{H}_{2} \mathrm{O}+\mathrm{O}_{2}$ & $1.45 \times 10^{16}$ & -1.0 & 0 \\
\hline 14 & $\mathrm{HO}_{2}+\mathrm{HO}_{2} \rightleftharpoons \mathrm{H}_{2} \mathrm{O}_{2}+\mathrm{O}_{2}$ & $3.02 \times 10^{12}$ & 0.0 & 1390 \\
\hline 15 & $\mathrm{H}_{2} \mathrm{O}_{2}+\mathrm{M} \rightleftharpoons \mathrm{OH}+\mathrm{OH}+\mathrm{M}^{a}$ & $1.20 \times 10^{17}$ & 0.0 & 45500 \\
\hline 16 & $\mathrm{H}_{2} \mathrm{O}_{2}+\mathrm{H} \rightleftharpoons \mathrm{H}_{2} \mathrm{O}+\mathrm{OH}$ & $1.00 \times 10^{13}$ & 0.0 & 3590 \\
\hline 17 & $\mathrm{H}_{2} \mathrm{O}_{2}+\mathrm{H} \rightleftharpoons \mathrm{H}_{2}+\mathrm{HO}_{2}$ & $4.79 \times 10^{13}$ & 0.0 & 7950 \\
\hline 18 & $\mathrm{H}_{2} \mathrm{O}_{2}+\mathrm{O} \rightleftharpoons \mathrm{OH}+\mathrm{HO}_{2}$ & $9.55 \times 10^{6}$ & 2.0 & 3970 \\
\hline 19 & $\mathrm{H}_{2} \mathrm{O}_{2}+\mathrm{OH} \rightleftharpoons \mathrm{H}_{2} \mathrm{O}+\mathrm{HO}_{2}$ & $7.08 \times 10^{12}$ & 0.0 & 1430 \\
\hline 20 & $C O+O+M \rightleftharpoons C O_{2}+M^{a}$ & $2.51 \times 10^{13}$ & 0.0 & -4540 \\
\hline 21 & $\mathrm{CO}+\mathrm{OH} \rightleftharpoons \mathrm{CO}_{2}+\mathrm{H}$ & $k=6.75 \times 10^{10}$ & $\exp (0.000907 \times T)$ & \\
\hline 22 & $\mathrm{CO}+\mathrm{O}_{2} \rightleftharpoons \mathrm{CO}_{2}+\mathrm{O}$ & $2.51 \times 10^{12}$ & 0.0 & 47690 \\
\hline 23 & $\mathrm{CO}+\mathrm{HO}_{2} \rightleftharpoons \mathrm{CO}_{2}+\mathrm{OH}$ & $6.03 \times 10^{13}$ & 0.0 & 22950 \\
\hline 24 & $H C O+M \rightleftharpoons C O+H+M^{a}$ & $1.86 \times 10^{17}$ & -1.0 & 17000 \\
\hline 25 & $\mathrm{HCO}+\mathrm{H} \rightleftharpoons \mathrm{CO}+\mathrm{H}_{2}$ & $7.24 \times 10^{13}$ & 0.0 & 0 \\
\hline 26 & $\mathrm{HCO}+\mathrm{O} \rightleftharpoons \mathrm{CO}+\mathrm{OH}$ & $3.02 \times 10^{13}$ & 0.0 & 0 \\
\hline 28 & $\mathrm{HCO}+\mathrm{O}_{2} \rightleftharpoons \mathrm{CO}+\mathrm{HO}_{2}$ & $4.17 \times 10^{13}$ & 0.0 & 0 \\
\hline
\end{tabular}

Note: Units are cal, mole, $\mathrm{cm}$, and $\mathrm{K}$. The third body efficiencies are $\mathrm{H}_{2}: 2.5, \mathrm{H}_{2} \mathrm{O}: 12.0$, $\mathrm{CO}_{2}: 3.8, \mathrm{CO}: 1.9$

The transport and thermodynamic properties are calculated using the same approach as used in the CHEMKIN II package to calculate thermal diffusivity, $\alpha$, thermal conductivity, $\lambda$, heat capacity at constant pressure, $c_{p}$, and viscocity, $\mu[45,46]$. These transport and thermodynamic properties are updated using external subroutines that were integrated into the OpemFOAM solver. 


\subsection{The Finite Volume Method}

The finite volume method (FVM) is an approach used to represent and model the NavierStokes partial differential equations. The Navier-Stokes PDEs are non-linear and coupled, which require computer assistance in order to mathematically solve scenarios that cannot be reduced due to complex geometries and boundary conditions. Consider a volume, $\mathcal{V}$, with surface $\mathcal{S}$. The integral conservation laws is written as:

$$
\frac{\partial}{\partial t} \int_{\mathcal{V}} \Phi d \mathcal{V}+\oint \vec{F} \cdot d \vec{S}=\int_{\mathcal{V}} Q d \mathcal{V}
$$

where $\Phi$ is some transported variable, e.g. mass, momentum, energy, species concentration, etc., $F$ is the flux of those variables located along the surface, and $Q$ is a source term. Specifically, the finite volume conservation law says that a complete understanding of the rate of change of a variable within a volume is dictated by the internal sources and the external fluxes at the boundaries. Defining the volume average of $\Phi$, as

$$
\bar{\Phi}=\frac{\int_{\mathcal{V}} \phi d \mathcal{V}}{\mathcal{V}}
$$

the integral conservation becomes

$$
\frac{\partial \bar{\Phi}}{\partial t}+\frac{\oint \vec{F} \cdot d \vec{S}}{\mathcal{V}}=\bar{Q}
$$

Considering a small, cartesian, volume element where the volume can be expressed as

$$
\mathcal{V}=\Delta x \Delta y \Delta z
$$

results in Equation 2.8 becoming

$$
\frac{\partial \bar{\Phi}}{\partial t}+\frac{\oint \vec{F} \cdot d \vec{S}}{\Delta x \Delta y \Delta z}=\bar{Q}
$$


For clarity, consider the conservation of a one-dimensional transported scalar across the finite volume cells depicted in Figure 2.1.

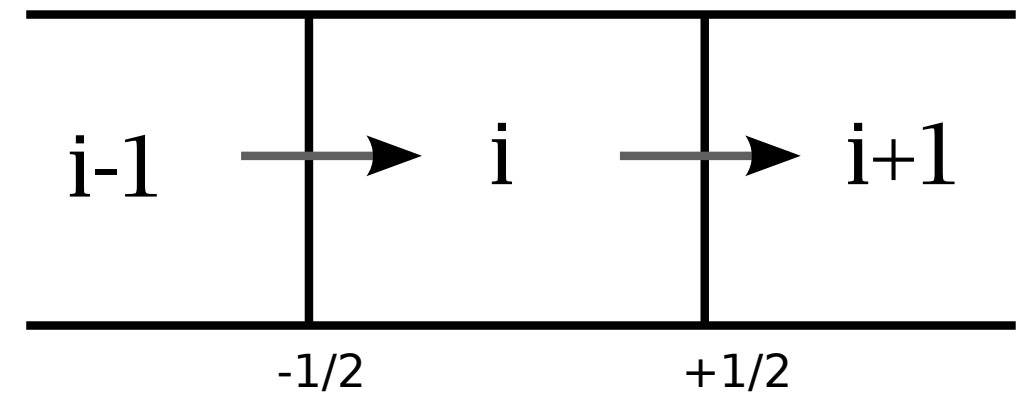

Figure 2.1: One dimensional finite volume diagram, where $i$ indicates the location in the particular dimension of interest.

In this configuration, two neighboring volumes at location $i+1$ and $i-1$ exist with internal values for velocity, $u$, and the transported quantity, $\Phi$. The second term of Equation 2.8 can be expressed as a sum of fluxes according to the normal $\vec{S}$,

$$
\frac{\oint \vec{F} \cdot d \vec{S}}{\Delta x \Delta y \Delta z}=\frac{\sum_{j}^{N}(\vec{F} \cdot \vec{S})_{j}}{\Delta x \Delta y \Delta z}=\frac{(F A)_{i+1 / 2}-(F A)_{i-1 / 2}}{\Delta x \Delta y \Delta z}=\frac{(F \Delta y \Delta z)_{i+\frac{1}{2}}-(F \Delta y \Delta z)_{i-\frac{1}{2}}}{\Delta x \Delta y \Delta z},
$$

where the $j$ subscript refers to the face and $A$ is the surface area. This volume is uniform, i.e. $\Delta x_{i+\frac{1}{2}}=\Delta x_{i-\frac{1}{2}}, \Delta y_{i+\frac{1}{2}}=\Delta y_{i-\frac{1}{2}}$, and $\Delta z_{i+\frac{1}{2}}=\Delta z_{i-\frac{1}{2}}$, and using the flux at a face approximation,

$$
F_{i \pm \frac{1}{2}}=\frac{(\bar{u} \bar{\Phi})_{i}+(\bar{u} \bar{\Phi})_{i \pm 1}}{2}
$$

leads to the central differencing approximation for fluxes,

$$
\frac{\oint \vec{F} \cdot d \vec{S}}{\Delta x \Delta y \Delta z}=\frac{F_{i+\frac{1}{2}}-F_{i-\frac{1}{2}}}{\Delta x}=\frac{(\bar{u} \bar{\Phi})_{i+1}-(\bar{u} \bar{\Phi})_{i-1}}{2 \Delta x} .
$$

For the sake of simplicity, the time derivative of $\Phi$ is approximated using explicit, first order discretization,

$$
\frac{\partial \bar{\Phi}}{\partial t}=\frac{\bar{\Phi}_{i}^{k+1}-\bar{\Phi}_{i}^{k}}{\Delta t}
$$


where superscript $k+1$ is the value of $\bar{\Phi}$ at one time step, $\Delta t$, later than instance $k$. Ignoring any sources, the finite volume method is used to find the estimated volume averaged quantity by

$$
\bar{\Phi}_{i}^{k+1}=\bar{\Phi}_{i}^{k}+\frac{\Delta t}{2 \Delta x}\left((\bar{u} \bar{\Phi})_{i+1}-(\bar{u} \bar{\Phi})_{i-1}\right)^{k} .
$$

The finite volume method gives an approximate solution for the conservation equations based on a series of neighboring volumes and their fluxes. In CFD, the fluid dynamic domain is split into several elemental volumes, usually referred to as cells, that make up the grid of the simulated geometry. By fixing the boundaries of this domain at some mathematical condition, such as fixed fluxes, the FVM is used to solve for the internal, volume averaged, values of the interested quantities.

Although the modeled PDEs are usually much more complex than the PDE that results in Equation 2.9, the same fundamental approach of conserving fluxes and sources can be applied to add diffusive Laplacian schemes, source terms (such as chemical or energy), and multi-dimensional fluxes (for two or three dimensional domains) into the finite volume equations. After all these terms are included, the finite volume approximation becomes

$$
A_{P} \bar{\Phi}_{i, P}^{n+1}+\sum_{L} A_{L} \bar{\Phi}_{i, L}^{n+1}=Q_{i}^{n}
$$

where $A_{P}$ is the coefficeint of the point of interest's volumetric average, $A_{L}$ are the coefficeints for the neighboring cells of the point of interest, $\sum_{L}$ represents summing over all these faces, and $Q_{i}^{k}$ is the source term. Here the $k+1$ denotes an implicitly treated term and $k$ represents an explicit term. A computer is then used to solve this linearized expression using linear-algebra methods by storing the coefficeints in matrix $\mathbb{A}$ and source terms in vector $\mathbb{Q}$,

$$
\mathbb{A} \vec{\Phi}-\overrightarrow{\mathbb{Q}}=0
$$

The finite volume method is often referred to as the weak form of these conservation laws because sharp discontinuities are allowed to exist and can usually be handled with ease 
by the simulation. This not only allows for physical phenomena like shock-waves, but also allows for initial guesses that have sharp curvatures at boundaries or within the domain. Eventually the simulations should eliminate any unwanted discontinuities and allow any physical sharp gradients. This is an advantage that the finite volume method has over other discretization techniques, such as finite differencing, which is considered the strong form of the conservation equations. These other techniques are much more unstable [47]. Despite the advantages of the FVM, certain instabilities can arise in simple flux differencing techniques, which requires the use of more advanced schemes [48-50]. These schemes aid in resolving any unwanted perturbations and assist in calculating stable solutions. The ease of multidimensional implementation, the inherent stability, and the ability to add additional chemistry and transport properties is the reason this research utilized the finite volume method.

\subsection{The OpenFOAM Computational Package}

An open-source computational fluid dynamics package, OpenFOAM (Open Field Operation and Manipulation), was used in this work to model the reacting Navier-Stokes conservation equations using the finite volume method. From the OpenFOAM website (www.openfoam.org):

OpenFOAM offers users complete freedom to customise and extend its existing functionality. It follows a highly modular code design in which collections of functionality (e.g. numerical methods, meshing, physical models, etc) are each compiled into their own shared library. Executable applications are then created that are simply linked to the library functionality. The OpenFOAM package includes over 80 solver applications that simulate specific problems in engineering mechanics and over 170 utility applications that perform pre- and post-processing tasks, e.g. meshing, data visualisation, etc.

The OpenFOAM package gives the unique ability for a programmer to quickly imple- 
ment various numerical models as well as use existing complex finite volume techniques to solve easily programmed conservation equations. For example, the equation

$$
\frac{\partial \rho \vec{v}}{\partial t}+\nabla \cdot(\rho \vec{v} \vec{v})-\nabla \cdot \mu \nabla \vec{v}=-\nabla p
$$

is implemented by:

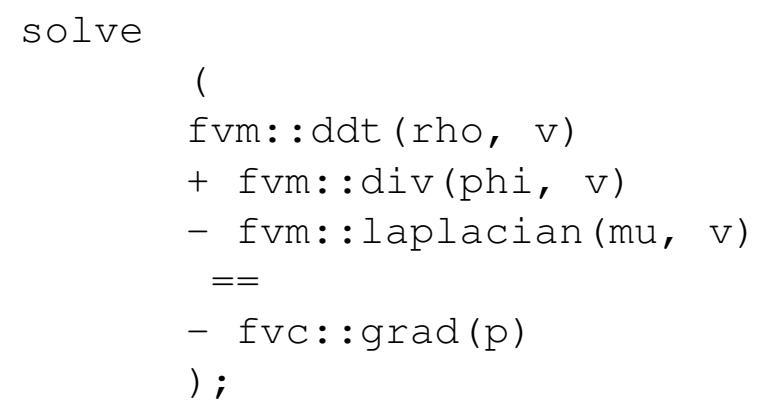

Where the variables $r h o, v, m u$, and $p$ are all scalar fields that are linked with the mesh of the computational domain, and phi is the array of fluxes at cell faces. OpenFOAM uses its operator class to perform the correct numerical vector calculus (ie. fvm: : div (phi, v) is the divergence of the fluxes combined with the velocity, v) on the scalar fields and carefully adds the algebraic quantities to a finite volume matrix. This matrix is determined by the implicit or explicit schemes as well as the spatial discretization technique and is solved using a variety of techniques and pre-conditioners. The ease of implementing any conservation equation and the ability to add extra terms and numerical methods reduces the time to create in-house codes for specific academic applications.

\subsection{Reacting Interface Boundary Conditions}

The approach used to model a reacting interface in this dissertation was guided by Forman Williams' book, Combustion Theory, Section 1.4 [51]. The approach outlined in the book is thorough; however, it does not take into account the application of a solid, reacting surface. 
This section covers the detailed derivation of the conservation equations at a reacting solid interface, which were implemented as independent subroutines in the OpenFOAM solver. The next section, 2.4.3 covers the pressure interface conditions.

Consider Figure 2.2, which shows an infinitesimal volume, $\mathcal{V}$, with height, $\Delta n$, normal vectors, $\vec{n}^{+}$and $\vec{n}^{-}$, and interface surface area, $\mathcal{A}_{i}$.

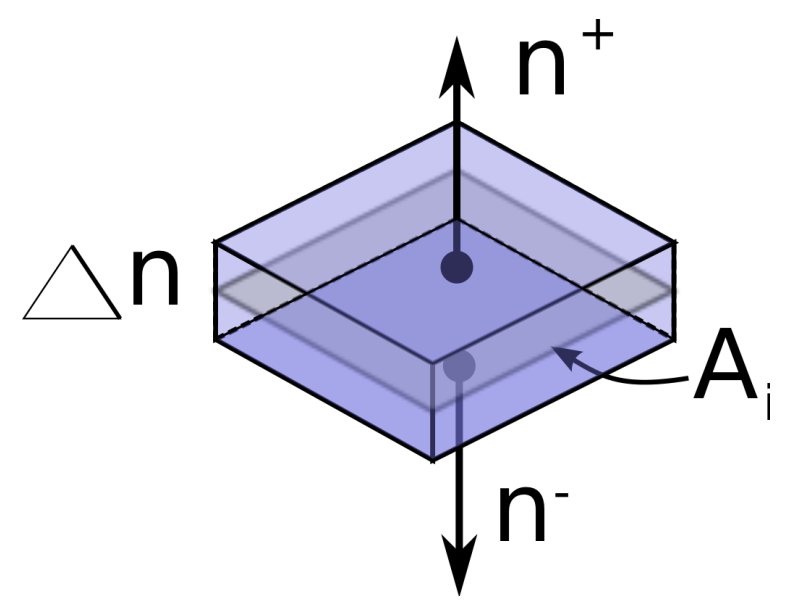

Figure 2.2: Infinitesimal volume with interface area $\mathcal{A}_{i}$, height $\Delta n$, and surface normal vectors, $\vec{n}$.

The conservation of mass, momentum, energy, and species for this volume are defined using the Reynolds transport thereom,

$$
\begin{aligned}
\frac{d}{d t} \int_{\mathcal{V}} \rho d \mathcal{V}+\int_{\mathcal{A}} \rho\left(\vec{v}-\frac{\partial \vec{x}}{\partial t}\right) \cdot \vec{n} d \mathcal{A} & =0 \\
\frac{d}{d t} \int_{\mathcal{V}} \rho \vec{v} d \mathcal{V}+\int_{\mathcal{A}} \rho \vec{v}\left(\vec{v}-\frac{\partial \vec{x}}{\partial t}\right) \cdot \vec{n} d \mathcal{A}= & -\int_{\mathcal{A}}(p+\mathbb{T}) \cdot \vec{n} d \mathcal{A} \\
& +\int_{\mathcal{V}} \rho \sum_{i=1}^{N} Y_{i} f_{i} d \mathcal{V} \\
\frac{d}{d t} \int_{\mathcal{V}} \rho\left(u+\frac{\vec{v} \cdot \vec{v}}{2}\right) d \mathcal{V}+\int_{\mathcal{A}} \rho\left(u+\frac{\vec{v} \cdot \vec{v}}{2}\right)\left(\vec{v}-\frac{\partial \vec{x}}{\partial t}\right) \cdot \vec{n} d \mathcal{A}= & -\int_{\mathcal{A}} \vec{v} \cdot(p+\mathbb{T}) \cdot \vec{n} d \mathcal{A} \\
& \left.+\int_{\mathcal{V}} \rho \sum_{i=1}^{N} Y_{i} f_{i} \cdot\left(\vec{V}_{i}+\vec{v}\right) d \mathcal{V}\right) \\
& -\int_{\mathcal{A}} \vec{q} \cdot \vec{n} d \mathcal{A} \\
\frac{d}{d t} \int_{\mathcal{V}} \rho Y_{i} d \mathcal{V}+\int_{\mathcal{A}} \rho Y_{i}\left(\vec{v}-\frac{\partial \vec{x}}{\partial t}+\overrightarrow{V_{i}}\right) \cdot \vec{n} d \mathcal{A}= & \int_{\mathcal{V}} w_{i} d \mathcal{V}
\end{aligned}
$$


Here, $\frac{d \vec{x}}{d t}$ is the velocity of the moving interface. In this derivation, $\frac{d \vec{x}}{d t}$ is assumed to be 0 as the physical frame of reference follows the regression ${ }^{2}$. Additionally, the body forces, $f_{i}$, and off diagonal pressure-tensor terms, $\mathbb{T}$, are assumed to be zero. The relations at the interface will be derived by taking the limit as $\Delta n \rightarrow 0$ and $\mathcal{V} \rightarrow 0$. At this limit, all peripheral faces disappear, leaving the surface integral to only concern the $n^{+}$and $n^{-}$ directional faces. Additionally, all volumetric densities become surface densities,

$$
\lim _{\mathcal{V} \rightarrow 0} \int_{\mathcal{V}} \rho d \mathcal{V}=\int_{\mathcal{A}} \rho^{\prime} d A
$$

and all source terms become surface source terms,

$$
\lim _{\mathcal{V} \rightarrow 0} \int_{\mathcal{V}} w d \mathcal{V}=\int_{\mathcal{A}} w^{\prime} d A
$$

As a consequence of $\Delta n \rightarrow 0$, the normal vectors, $\vec{n}^{+}$and $\vec{n}^{-}$, become equal and opposite,

$$
\vec{n}^{+}=-\vec{n}^{-} .
$$

With these assumptions, Equations 2.17 and 2.16 become

\footnotetext{
${ }^{2}$ This can also be viewed as grouping $\vec{v}+\frac{\partial \vec{x}}{\partial t}$ together.
} 


$$
\begin{aligned}
\frac{d}{d t} \int_{\mathcal{A}_{i}} \rho^{\prime} d \mathcal{A}_{i}+\int_{\mathcal{A}_{i}}\left(\rho^{+} v^{+}-\rho^{-} v^{-}\right) d \mathcal{A}_{i} & =0, \\
\frac{d}{d t} \int_{\mathcal{A}_{i}} \rho^{\prime} \vec{v} d \mathcal{A}_{i}+\int_{\mathcal{A}} \rho^{+} \vec{v}^{+} v^{+}-\rho^{-} \vec{v}^{-} v^{-} d \mathcal{A}_{i} & =\ldots \\
-\int_{\mathcal{A}}\left(p^{+}-p^{-}\right) d \mathcal{A}_{i}, & \\
\frac{d}{d t} \int_{\mathcal{A}_{i}} \rho^{\prime}\left(u+\frac{\vec{v} \cdot \vec{v}}{2}\right) d \mathcal{A}_{i}+\int_{\mathcal{A}_{i}} \rho^{+}\left(u+\frac{\vec{v} \cdot \vec{v}}{2}\right)^{+} v^{+}-\rho^{-}\left(u+\frac{\vec{v} \cdot \vec{v}}{2}\right)^{-} v^{-} d \mathcal{A}_{i} & =\ldots \\
-\int_{\mathcal{A}_{i}}\left(v p^{+}-v p^{-}\right) d \mathcal{A}_{i}-\int_{\mathcal{A}_{i}}\left(q^{+}-q^{-}\right) d \mathcal{A}_{i}, & \\
\frac{d}{d t} \int_{\mathcal{A}_{i}} \rho^{\prime} Y_{i} d \mathcal{A}_{i}+\int_{\mathcal{A}_{i}} \rho^{+} Y_{i}^{+}\left(v^{+}+V_{i}^{+}\right)-\rho^{-} Y_{i}^{-}\left(v^{-}+V_{i}^{-}\right) d \mathcal{A}_{i} & =\int_{\mathcal{A}_{i}} w_{i}^{\prime} d \mathcal{A}_{i} .
\end{aligned}
$$

Here, the + and - superscripts denote the dot product of the considered vector in the direction of $n^{+}$and $n^{-}$respectively. For instance, $v^{+}$is $\vec{v} \cdot \vec{n}^{+}$and $q^{+}$is $\vec{q} \cdot \vec{n}^{+}$. Figure 2.3 represents the conservation at an interface for a reacting solid surface.

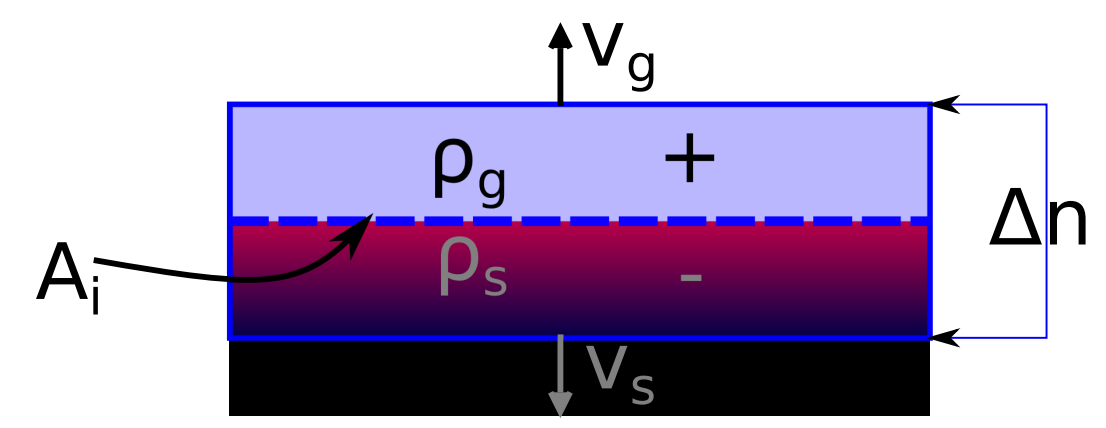

Figure 2.3: Infinitesimal volume where + is the gaseous portion and - is the solid portion.

The + portion of the volume is the gaseous side and the - portion is on the solid side. It is assumed that a particular gaseous species, $Y_{i}$, cannot exist within the solid surface and thus $Y_{i}^{-}=0$. For clarity, the solid region properties are changed to subscript $s$ and gaseous region properties are changed to subscript $g$. Using these identities and rearranging all terms to be under one integral, $\int_{\mathcal{A}_{i}}$, the conservation equations are further reduced, 


$$
\begin{gathered}
\int_{\mathcal{A}_{i}}\left(\frac{d \rho^{\prime}}{d t}+\rho_{g} v_{g}-\rho_{s} v_{s}\right) d \mathcal{A}_{i}=0 \\
\int_{\mathcal{A}_{i}}\left(\frac{\partial \rho^{\prime} \vec{v}}{\partial t}+\rho_{g} \vec{v}_{g} v_{g}-\rho_{s} \vec{v}_{s} v_{s}-p_{g}+p_{s}\right) d \mathcal{A}_{i}=0 \\
\int_{\mathcal{A}_{i}}\left(\frac{\partial \rho^{\prime}\left(u+\frac{\vec{v} \cdot \vec{v}}{2}\right)}{\partial t}+\rho_{g}\left(u+\frac{\vec{v} \cdot \vec{v}}{2}\right)_{g} v_{g}-\rho_{s}\left(u+\frac{\vec{v} \cdot \vec{v}}{2}\right)_{s} v_{s}+v_{g} p_{g}-v_{s} p_{s}+q_{g}-q_{s}\right) d \mathcal{A}_{i}=0, \\
\int_{\mathcal{A}_{i}}\left(\frac{\partial \rho^{\prime} Y_{i}}{\partial t}+\rho_{g} Y_{i}\left(v_{g}+V_{i}\right)-w_{i}^{\prime}\right) d \mathcal{A}_{i}=0 .
\end{gathered}
$$

All these integrals are zero, and as such, in the limit of small area, $\mathcal{A}_{i} \rightarrow 0$, the integrands must also be zero ${ }^{3}$. Realizing this leads to the partial differential equations for a reacting solid interface,

$$
\begin{gathered}
\frac{d \rho^{\prime}}{d t}+\rho_{g} v_{g}-\rho_{s} v_{s}=0 \\
\frac{\partial \rho^{\prime} \vec{v}}{\partial t}+\rho_{g} \vec{v}_{g} v_{g}-\rho_{s} \vec{v}_{s} v_{s}-p_{g}+p_{s}=0 \\
\left.\frac{\partial \rho^{\prime} \cdot \vec{v}}{2}\right) \\
\frac{\partial t}{\partial t}+\rho_{g}\left(u+\frac{\vec{v} \cdot \vec{v}}{2}\right)_{g} \rho_{g} Y_{i}\left(v_{g}+V_{i}\right)-w_{i}^{\prime}=0
\end{gathered}
$$

Here, $w_{i}^{\prime}$ is the surface mass production rate for species $i$, that is determined by the heterogeneous reactions at a surface. For carbon surfaces, the relationships that give $w_{i}^{\prime}$ are covered in 2.5. Assuming that the expressions for $w_{i}^{\prime}$ are available (for carbon surfaces they are found experimentally), an expression for $v_{g}$ is extracted by summing Equation 2.20 for

\footnotetext{
${ }^{3}$ This conclusion can be reached in many ways. Another reasoning is that the derivation is done for arbitraty $\mathcal{A}_{i}$. Therefore, if the integral is zero for any $\mathcal{A}_{i}$, then the integrand must be zero.
} 
all species,

$$
\sum_{i=1}^{N}\left(\frac{d}{d t}\left(\rho^{\prime} Y_{i}\right)+\rho Y_{i}\left(v_{g}+V_{i}\right)\right)=\sum_{i=1}^{N} w_{i}^{\prime}
$$

This summation must lead to the continuity equation. By applying the steady state condition, $\sum_{i=1}^{N} \frac{d \rho^{\prime} Y_{i}}{d t}=$ 0 , and using the mixture identities of $\sum_{i=1}^{N} Y_{i} V_{i}=0$ and $\sum_{i=1}^{N} Y_{i}=1$, a relation for $v_{g}$ is found,

$$
\rho_{g} v_{g}=\sum_{i=1}^{N} w_{i}^{\prime} \rightarrow v_{g}=\frac{\sum_{i=1}^{N} w_{i}^{\prime}}{\rho_{g}}
$$

Generally, for catalytic reactions, $\sum_{i=1}^{N} w_{i}^{\prime}=0$; however, for a reacting surface that contributes mass to the flow, like a heated carbon surfaces, mass is added to the system, i.e. $\sum_{i=1}^{N} w_{i}^{\prime} \neq 0$. Relation 2.22 is used to calculate the normal velocity of the surface instead of the mometum conservation. This approach guarantees a unique value for blowing velocity. In addition, the mass loss rate of the surface, $\dot{m}_{s}=\rho_{s} v_{s}$, is related to the heterogeneous rates by using the continuity equation, Equation 2.17, through transitive equality,

$$
\underbrace{\frac{\partial \rho^{\prime}}{\partial t}}_{\text {steady state }}+\rho_{g} v_{g}-\rho_{s} v_{s}=0 \rightarrow \rho_{g} v_{g}=\rho_{s} v_{s}=\sum_{i=1}^{N} w_{i}^{\prime}
$$

The following subsections will cover how PDEs 2.17, 2.18, and 2.20 are implemented to solve for the blowing velocity, species, and thermal interface boundary conditions at the reacting surface. Section 2.4 .3 covers how pressure is specified at the surface, which gives a complete set of boundary conditions for these reacting surfaces.

\subsubsection{Species Conservation at a Reacting Solid Surface}

For this model the species conservation at the reacting surface is reduced to

$$
\frac{\partial \rho^{\prime} Y_{i}}{\partial t}+\rho Y_{i}\left(v+V_{i}\right)-w_{i}^{\prime}=0
$$


The notation for this expression is changed to use subscript $n$ for any normal direction quantity. The species diffusion velocity is assumed according to a Fickian model, $V_{i}=$ $-\frac{1}{Y_{i}} D_{i} \nabla Y i$, where $D_{i}$ is the mixture-averaged diffusion coeffeceint. Using this assumption and changing the notation, the species conservation becomes

$$
\frac{\partial \rho^{\prime} Y_{i}}{\partial t}+\rho Y_{i} v_{n}-\rho D_{i} \frac{\partial Y_{i}}{\partial n}-w_{i}^{\prime}=0
$$

Equation 2.25 contains a transient term, $\frac{\partial \rho^{\prime} Y_{i}}{\partial t}$, convective term, $\rho Y_{i} v_{n}$, diffusive term, $\rho D_{i} \frac{\partial Y_{i}}{\partial n}$, and mass production term, $w_{i}^{\prime}$. This PDE is solved via a time-splitting technique, where the surface species quantities are updated between fluid dynamic and homogeneous chemistry time integrations.

Consider the finite volume element located next to the reacting surface as depicted in Figure 2.4.

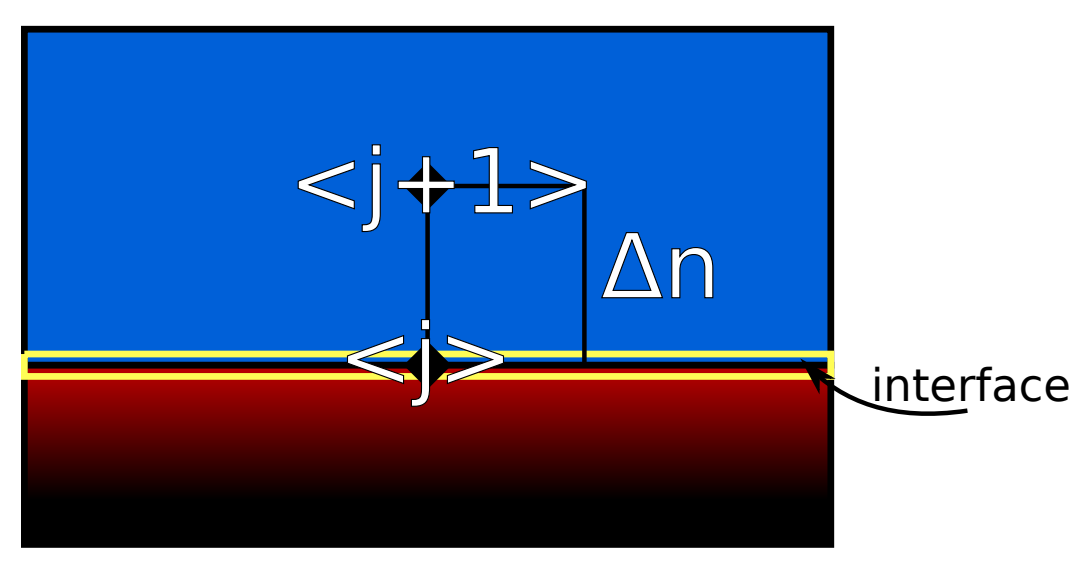

Figure 2.4: Computational cell next to reacting surface.

OpenFOAM solves for cell-centered averaged values, which are located a normal distance of $\Delta n$ away from the surface. The cell centered values will be denoted with a subscript $\langle j+1\rangle$ and boundary values with subscript $\langle j\rangle$. Using these values, Equation 2.25 can be discretized. For now, consider the time transient term as explicitly discretized,

$$
\frac{\partial\left(\rho^{\prime} Y_{i}\right)}{\partial t}=\frac{\left(\rho^{\prime} Y_{i}\right)_{\langle j\rangle}^{k+1}-\left(\rho^{\prime} Y_{i}\right)_{\langle j\rangle}^{k}}{\Delta t_{s i m}}
$$


Where $\Delta t_{\text {sim }}$ is the simulation time step and the $k$ superscript denotes the discrete place in time. The integration of this transient term is covered at the end of this section. The surface density, $\rho^{\prime}$, is approximated using the height of the finite volume cell, $\rho^{\prime}=\rho_{\langle j+1\rangle} 2 \Delta n$. The convective term is calculated using previous time step values, $\rho^{k} Y_{i}^{k} v_{n}^{k}$, where the blowing velocity is calculated using the continuity relation from Equation 2.22,

$$
\rho_{g} v_{g}=\sum_{i=1}^{N} w_{i}^{\prime} \rightarrow v_{g}^{k}=\frac{\sum_{i=1}^{N}{w_{i}^{\prime}}^{k}}{\rho_{g}}
$$

The diffusive term is approximated using first-order, upwind finite differencing of the cell centered value, $\langle j+1\rangle$, and boundary value, $\langle j\rangle$,

$$
\rho D_{i} \frac{\partial Y_{i}}{\partial n}=\rho_{\langle j\rangle} D_{i,\langle j\rangle} \frac{Y_{i,\langle j+1\rangle}-Y_{i,\langle j\rangle}}{\Delta n} .
$$

Finally, the mass production term is calculated using the methods described in Section 2.5. Substituting these terms into 2.25 yields the full discretization of the species conservation at the reacting surface,

$$
\frac{\left(\rho^{\prime} Y_{i}\right)_{\langle j\rangle}^{k+1}-\left(\rho^{\prime} Y_{i}\right)_{\langle j\rangle}^{k}}{\Delta t_{s i m}}+\rho_{\langle j\rangle}^{k} Y_{i, j j\rangle}^{k} v_{n,\langle j\rangle}^{k}-\rho_{\langle j\rangle}^{k} D_{i,\langle j\rangle}^{k} \frac{Y_{i,\langle j+1\rangle}^{k}-Y_{i,\langle j\rangle}^{k}}{\Delta n}-w_{i}^{\prime k}=0 .
$$

Solving for the $k+1$ term gives a linearized expression for the surface species change in time,

$$
\left(\rho^{\prime} Y_{i}\right)_{\langle j\rangle}^{k+1}=\left(\rho^{\prime} Y_{i}\right)_{\langle j\rangle}^{k}-\Delta t_{s i m}\left(\rho_{\langle j\rangle}^{k} Y_{i,\langle j\rangle}^{k} v_{n,\langle j\rangle}^{k}-\rho_{\langle j\rangle}^{k} D_{i,\langle j\rangle}^{k} \frac{Y_{i,\langle j+1\rangle}^{k}-Y_{i,\langle j\rangle}^{k}}{\Delta n}-w_{i}^{\prime k}\right) .
$$

The choice of $\Delta t_{\text {sim }}$ is ideally the fluid dynamic time scale, $\Delta t_{\text {fluid }}$. As previously mentioned, the gas phase conservation equations are solved according to a time step that is chosen based on a CFL criteria number. This gives a $\Delta t_{\text {fluid }}$ on the order of $10^{-6} \mathrm{~s}$. In developing this subroutine, it was found that this fluid dynamic time-scale is too large and 
resulted in values of $\left(Y_{i}\right)_{\langle j\rangle}^{k+1}$ that were not physical, i.e. $\left(Y_{i}\right)_{\langle j\rangle}^{k+1}<0$. Figure 2.5 shows an illustration of how Equation 2.27 is semi-implicitly marched in time from initial value $Y_{0}$ to achieve a stable, physical, and eventually steady-state result, $Y_{s s}$.

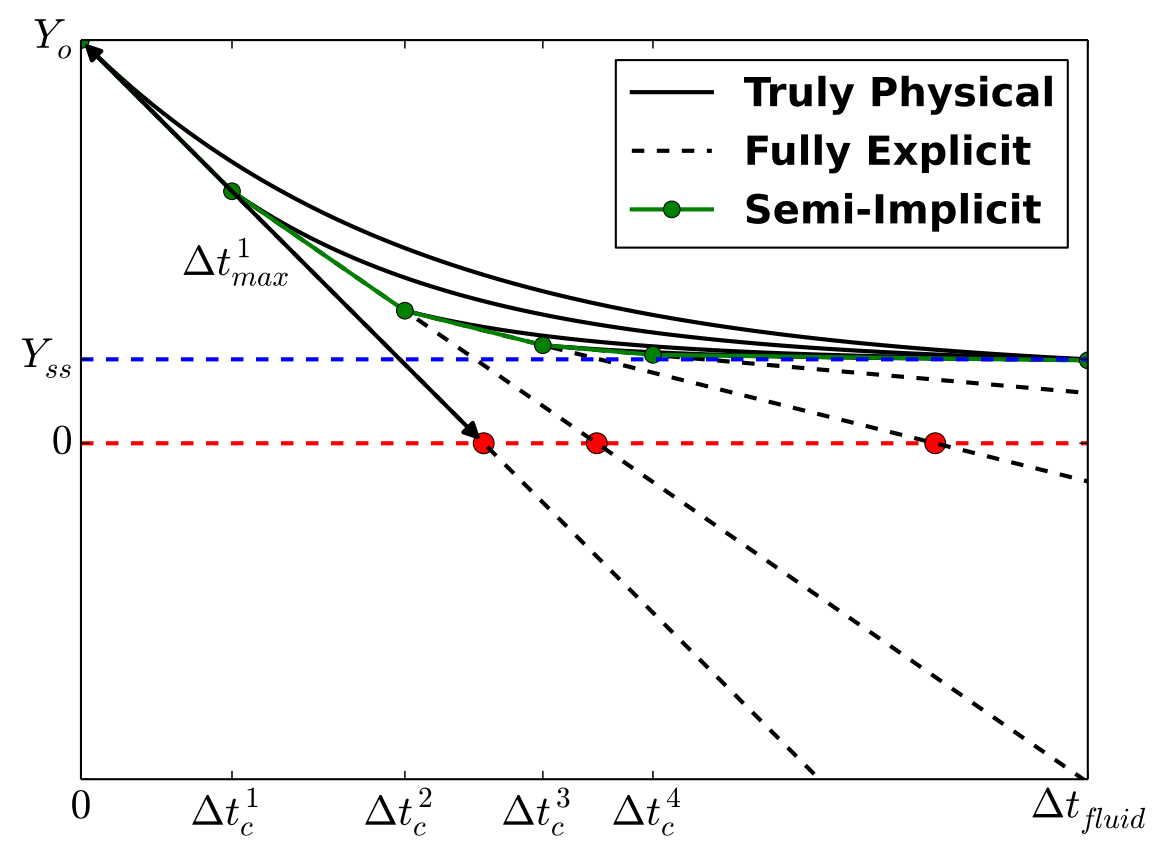

Figure 2.5: Integration of species conservation at an interface using semi-implicit methods

At initially high species surface quantities, $Y_{0}$, the mass production source, diffusive, and convective terms make the $\left(\rho^{k} Y_{i}^{k} v_{n}^{k}-\rho_{\langle j\rangle}^{k} D_{i,\langle j\rangle}^{k} \frac{Y_{i,\langle j+1\rangle}^{k}-Y_{i,\langle j\rangle}^{k}}{\Delta n}-\dot{s}_{i}^{k}\right)$ term of Equation 2.27 so large that, if $\Delta t_{\text {fluid }}$ is used, the value of the species falls below zero. The fully explicit implementation using $\Delta t_{\text {fluid }}$ is illustrated by the black dashed lines in Figure 2.5. To correct this, the critical time step, $\Delta t_{\max }$, is found as the time the explicit method takes to give a value of 0 . Next, a new time scale is calculated as a fraction of the critical time scale, $\Delta t_{c}=\alpha \Delta t_{\max }$, where $0<\alpha<1$. This new time scale, $\Delta t_{c}$, is used as $\Delta t_{\text {sim }}$ which gives new values for the mass production, diffusive, and convective terms. This is done for several integrations, $\Delta t_{c}^{1}, \Delta t_{c}^{2}, \Delta t_{c}^{3}, \Delta t_{c}^{4} \ldots$, until the sum of these time steps becomes $\Delta t_{\text {fluid }}$ and the final value, $Y_{s s}$, is calculated before the next iteration of the gas-phase conservation equations. This integration is split in time from the other conser- 
vation equations and is referred to as a semi-implicit ${ }^{4}$ method. This semi-implicit method is illustrated with a green line in Figure 2.5. The black lines in Figure 2.5 illustrate how this conservation equation would actually behave if all time scales and changes could be captured (a perfect integration scheme). Despite the discrepancies during the integration, this work is interested in a steady state value, which is consistent in Figure 2.5.

\subsubsection{Energy Conservation at a Reacting Interface Solid Surface}

Consider Equation 2.15 for energy at a reacting surface,

$$
\frac{\partial \rho^{\prime}\left(u+\frac{\vec{v} \cdot \vec{v}}{2}\right)}{\partial t}+\rho_{g}\left(u+\frac{\vec{v} \cdot \vec{v}}{2}\right)_{g} v_{g}-\rho_{s}\left(u+\frac{\vec{v} \cdot \vec{v}}{2}\right)_{s} v_{s}+v_{g} p_{g}-v_{s} p_{s}+q_{g}-q_{s}=0
$$

For low-speed flows, the kinetic energy term, $\frac{v \cdot v}{2}$, is small in comparison to internal energy, $u$, and can be neglected [51]. It is also convenient to write the energy conservation in terms of enthalpy, ${ }^{5} u=h-\frac{p}{\rho}$. The elimination of kinetic energy and inclusion of enthalpy gives

$$
\frac{d}{d t}\left(\rho^{\prime}\left(h-\frac{p}{\rho^{\prime}}\right)\right)+\rho_{g}\left(h_{g}-\frac{p_{g}}{\rho_{g}}\right) v_{g}-\rho_{s}\left(h_{s}-\frac{p_{s}}{\rho_{s}}\right) v_{s}+v_{g} p_{g}-v_{s} p_{s}+q_{g}-q_{s}=0 .
$$

Through further manipulation,

$$
\begin{aligned}
\frac{\partial \rho^{\prime} h}{\partial t}-\frac{\partial p}{\partial t}+\rho_{g} h_{g} v_{g}-p_{g} v_{g}-\rho_{s} h_{s} v_{s}+p_{s} v_{s}+v_{g} p_{g}-v_{s} p_{s}+q_{g}-q_{s} & =0, \\
\frac{\partial \rho^{\prime} h}{\partial t}-\underbrace{\partial^{\partial t}}_{\text {No acoustics }}+\rho_{g} h_{g} v_{g}-p_{g} \forall_{g}-\rho_{s} h_{s} v_{s}+p_{s} \forall_{s}+v_{g} \not_{g}-v_{s} p_{s}+q_{g}-q_{s} & =0, \\
\frac{\partial \rho^{\prime} h}{\partial t}+\rho_{g} h_{g} v_{g}-\rho_{s} h_{s} v_{s}+q_{g}-q_{s} & =0,
\end{aligned}
$$

\footnotetext{
${ }^{4} \mathrm{~A}$ fully-implicit scheme would include calculating the interior conservation equations. The term semiimplicit is used as the right hand side of the discretized equation are updated during the integration.

${ }^{5}$ Any time derivative terms contain a $\rho^{\prime}$ as the original integration was volumetric.
} 
several terms can be eliminated and time transient terms of pressure neglected as acoustics are not important (and cause instabilities) for steady-state problems. The first term's enthalpy can be either the solid or gas phase enthalpy, as

$$
\rho^{\prime} h=\lim _{g \rightarrow i} \rho_{g}^{\prime} h_{g}=\lim _{s \rightarrow i} \rho_{s}^{\prime} h_{s}
$$

must be true for the continuous system [52]. Here, the $g \rightarrow i$ and $s \rightarrow i$ refers to the limit of approaching the interface from the gas and solid regions respectively. For this model, the gas enthalpy will be used. It is now convenient to rewrite the enthalpy in terms of species specific sensible enthalpy and creation enthalpy,

$$
h=h^{s}+h^{c}=\sum_{i=1}^{N} Y_{i}(\underbrace{\int_{T_{o}}^{T_{g}} c_{p, i} d T}_{\text {Sensible Enthalpy, } h_{i}^{s}}+\underbrace{h_{i}^{c}}_{\text {Creation Enthalpy }}) .
$$

By multiplying the species conservation equation, Eq. 2.24 , by $h_{i}^{c}$, summing over all species,

$$
\sum_{i=1}^{N} \frac{\partial \rho^{\prime} Y_{i}}{\partial t} h_{i}^{c}+Y_{i} h_{i}^{c} \rho_{g} v_{g}+h_{i}^{c} Y_{i} \rho V_{i}-w_{i}^{\prime} h_{i}^{c}=0,
$$

and subtracting from 2.29 ,

$$
\underbrace{\frac{\partial \rho_{g}^{\prime} h_{g}}{\partial t}}_{\mathrm{A}}+\underbrace{h_{g} \rho_{g} v_{g}}_{B}-h_{s} \rho_{s} v_{s}+\underbrace{q_{g}}_{\mathrm{C}}-q_{s}-\sum_{i=1}^{N} \underbrace{\frac{\partial \rho^{\prime} Y_{i}}{\partial t} h_{i}^{c}}_{\mathrm{A}}+\underbrace{Y_{i} h_{i}^{c} \rho v_{g}}_{B}+\underbrace{h_{i}^{c} Y_{i} \rho V_{i}}_{\mathrm{C}}-w_{i}^{\prime} h_{i}^{c}=0,
$$


an expression based on sensible enthalpy is obtained by collecting terms marked with an A,

$$
\begin{aligned}
\frac{\partial \rho_{g}^{\prime} h_{g}}{\partial t}-\sum_{i=1}^{N} \frac{\partial \rho_{g}^{\prime} Y_{i}}{\partial t} h_{i}^{c} & =\frac{\partial}{\partial t}\left(\rho_{g}^{\prime} h_{g}^{s}\right)+\frac{\partial}{\partial t}\left(\rho_{g}^{\prime} h_{g}^{c}\right)-\sum_{i=1}^{N} \frac{\partial}{\partial t}\left(\rho_{g}^{\prime} Y_{i}\right) h_{i}^{c}, \\
& =\frac{\partial}{\partial t}\left(\rho_{g}^{\prime} h_{g}^{s}\right)+\frac{\partial}{\partial t}\left(\rho^{\prime} \sum_{i=1}^{N} Y_{i} h_{i}^{c}\right)-\sum_{i=1}^{N} \frac{\partial}{\partial t}\left(\rho_{g}^{\prime} Y_{i}\right) h_{i}^{c}, \\
& =\frac{\partial}{\partial t}\left(\rho_{g}^{\prime} h_{g}^{s}\right)+\sum_{i \neq 1}^{N} \rho_{g}^{\prime} Y_{i} \frac{\partial}{\partial t}\left(h_{i}^{c}\right)+\sum_{i \neq 1}^{N} \frac{\partial}{\partial t}\left(\rho_{g}^{\left.Y_{i}\right) h_{i}^{c}}-\sum_{i \neq 1}^{N} \frac{\partial}{\partial t}\left(\rho_{g}^{\prime} Y_{i}\right) h_{i}^{c},\right.
\end{aligned}
$$

as well as the terms marked with a B,

$$
\begin{aligned}
h_{g} \rho_{g} v_{g}-\sum_{i=1}^{N} Y_{i} h_{i}^{c} \rho_{g} v_{g} & =\left(\sum_{i=1}^{N} Y_{i} h_{i}^{s}+\sum_{i=1}^{N} Y_{i} h_{i}^{c}\right) \rho_{g} v_{g}-\rho_{g} v_{g} \sum_{i=1}^{N} Y_{i} h_{i}^{c} \\
& =\rho_{g} v_{g} \sum_{i=1}^{N} Y_{i} h_{i}^{s}+\rho_{g} v_{g} \sum_{i=1}^{N} Y_{i} h_{i}^{c}-\rho_{g} v_{g} \sum_{i=1}^{N} Y_{i} h_{i}^{c}, .
\end{aligned}
$$

The gaseous energy flux, $q_{g}$, must be discussed before addressing terms marked with a C. This energy flux typically contains conduction, species specific thermal mass diffusion, and radiation. However, for this particular derivation, a source term, $\mathcal{L}$, for energy delivered to the surface must also be included. In most applications, the surface is heated through various mechanisms, from heated air in coal bed combustion to the externally heated surfaces of catalytic combustion. This dissertation concerns modeling laser-induced carbon surface reactions, for which there is no term for laser effluence in this derivation so far. With this term included, the energy mass flux is

$$
q_{g}=-\lambda \frac{\partial T}{\partial n}+\sum_{i=1}^{N} \rho_{g} h_{i} Y_{i} V_{i}+q_{R}-\mathcal{L}
$$

where $\lambda \frac{\partial T}{\partial n}$ is the conduction term, $\sum_{i=1}^{N} \rho_{g} h_{i} Y_{i} V_{i}$ is the species specific thermal mass diffusion, $q_{R}$ is radiation flux, and $\mathcal{L}$ is heat flux from an external source, such as laser effluence. 
Finally, terms labeled with a $\mathrm{C}$ can be reduced,

$$
\begin{aligned}
-\lambda \frac{\partial T}{\partial n}+\sum_{i=1}^{N} \rho_{g} h_{i} Y_{i} V_{i}+q_{R}-\mathcal{L}-\sum_{i=1}^{N} \rho_{g} V_{i} Y_{i} h_{i}^{c}= & -\lambda \frac{\partial T}{\partial n}+\sum_{i=1}^{N} \rho_{g} h_{i}^{c} Y_{i} V_{i}+\sum_{i=1}^{N} \rho_{g} h_{i}^{s} Y_{i} V_{i} \\
& +q_{R}-\mathcal{L}-\sum_{i=1}^{N} \rho_{g} V_{i} Y_{i} h_{i}^{c} \\
= & -\lambda \frac{\partial T}{\partial n}+\sum_{i=1}^{N} \rho_{g} h_{i}^{s} Y_{i} V_{i}+q_{R}+\mathcal{L} .
\end{aligned}
$$

Through collecting these terms, an expression for sensible enthalpy conservation at interface is found,

$$
\underbrace{\frac{\partial}{\partial t}\left(\rho_{g}^{\prime} h_{g}^{s}\right)}_{\text {Time transient term }}+\underbrace{h_{g}^{s} \rho_{g} v_{g}-\lambda \frac{\partial T}{\partial n}+\sum_{i=1}^{N} \rho_{g} h_{i}^{s} Y_{i} V_{i}+q_{R}-\mathcal{L}-\sum_{i=1}^{N} w_{i}^{\prime} h_{i}^{c}}_{\text {Gaseous region }}=\underbrace{h_{s} \rho_{s} v_{s}+q_{s}}_{\text {Solid region }}
$$

The solid region's two terms can be reduced by realizing that

$$
\rho_{s} v_{s} h_{s}=\sum_{i=1}^{N} w_{i}^{\prime} h_{s}
$$

and

$$
q_{s}=-\lambda_{s} \frac{\partial T}{\partial n} .
$$

Here, Equation 2.35 comes from heat conduction through a solid material as no other heat fluxes into the solid, e.g. radiation or species mass fluxes, exist. Substituting Equations 2.34 and 2.35 into Equation 2.33 gives a conservation equation almost purely in terms to of sensible enthalpy,

$$
\frac{\partial}{\partial t}\left(\rho_{g}^{\prime} h_{g}^{s}\right)+h_{g}^{s} \rho_{g} v_{g}-\lambda \frac{\partial T}{\partial n}+\sum_{i=1}^{N} \rho_{g} h_{i}^{s} Y_{i} V_{i}+q_{R}-\mathcal{L}-\sum_{i=1}^{N} w_{i}^{\prime} h_{i}^{c}=h_{s} \sum_{i=1}^{N} w_{i}^{\prime}-\lambda_{s} \frac{\partial T}{\partial n}
$$


To further assist, using the chain rule in the definition of enthalpy of an ideal gas, Equation 2.31 , the partial of enthalpy is expressed as

$$
\partial h^{s}=c_{p} \partial T \rightarrow \frac{1}{c_{p}} \partial h^{s}=\partial T
$$

Using this understanding, the sensible enthalpy conservation at a heated reacting interface is finally written as

$$
\frac{\partial}{\partial t}\left(\rho_{g}^{\prime} h_{g}^{s}\right)+h_{g}^{s} \rho_{g} v_{g}-\frac{\lambda_{g}}{c_{p, g}} \frac{\partial h_{g}^{s}}{\partial n}+\sum_{i=1}^{N} \rho_{g} h_{i}^{s} Y_{i} V_{i}+q_{R}+\mathcal{L}-\sum_{i=1}^{N} w_{i}^{\prime} h_{i}^{c}=h_{s} \sum_{i=1}^{N} w_{i}^{\prime}-\frac{\lambda_{s}}{c_{p, s}} \frac{\partial h_{s}^{s}}{\partial n}
$$

OpenFOAM solves for sensible enthalpy which makes $h_{g}^{s}$ and its derivatives accessible. Coupling the easily found enthalpy conditions with surface reaction subroutines makes this an easy-to-implement derived boundary condition for the reacting flow solver.

Complications arise when considering the heat transfer into the solid, represented by the term $\frac{\lambda_{s}}{c_{p, s}} \frac{\partial h_{s}^{s}}{\partial n}$. The entire solid domain must be simulated in order to determine this conduction term. For carbon surfaces, Thakre and Yang addressed this term by including the analytical solution for thermal diffusion in an annular material, the application being that of a graphite rocket nozzle. They found that the inclusion of this term had little effect, and their results showed good agreement with experiments when it was neglected [53]. Additionally, packed coal bed researchers, Reinelt et al. [18], modeled the conjugate heat transfer in a packed coal bed and concluded that the temperature profile within a solid substrate is rather flat, which leads to the conclustion $\frac{\partial T}{\partial n}=0 \rightarrow \frac{\partial h_{s}^{s}}{\partial n}=0$. For now, this term is treated explicitly as $q_{c, s}$, where the $c, s$ subscript stands for conduction into the solid. In Chapters 3 and $4, q_{c, s}$ is neglected with a pinned surface temperature profile, but Chapter 5 will discuss in further detail the approaches necessary to model the conjugate heat transfer problem.

Discretizing Equation 2.37 the same way as the species conservation at an interface 
gives an expression that is solved using the same previously mentioned semi-implicit method,

$$
\begin{array}{r}
\frac{\left(\rho h^{s}\right)_{\langle j\rangle}^{k+1}-\left(\rho h^{s}\right)_{\langle j\rangle}^{k}}{\Delta t_{s i m}}+\left(h_{\langle j\rangle}^{s} \rho_{\langle j\rangle} v_{\langle j\rangle}-\frac{\lambda_{\langle j\rangle}}{c_{p,\langle j\rangle}} \frac{h_{\langle j+1\rangle}^{s}-h_{\langle j\rangle}^{s}}{\Delta n}+\sum_{i=1}^{N} \rho_{\langle j\rangle} h_{i,\langle j\rangle}^{s} Y_{i,\langle j\rangle} V_{i,\langle j\rangle} \ldots\right. \\
\left.+q_{R,\langle j\rangle}+\mathcal{L}_{\langle j\rangle}-\sum_{i=1}^{N} w_{i,\langle j\rangle}^{\prime} h_{i,\langle j\rangle}^{c}\right)^{k}=\left(h_{s,\langle j\rangle} \sum_{i=1}^{N} w_{i,\langle j\rangle}^{\prime}-q_{c, s,\langle j\rangle}\right)^{k}, \\
\left(\rho h^{s}\right)_{\langle j\rangle}^{k+1}=\left(\rho h^{s}\right)_{\langle j\rangle}^{k}+\Delta t_{s i m}\left(-h_{\langle j\rangle}^{s} \rho_{\langle j\rangle} v_{\langle j\rangle}+\frac{\lambda_{\langle j\rangle}}{c_{p,\langle j\rangle}} \frac{h_{\langle j+1\rangle}^{s}-h_{\langle j\rangle}^{s}}{\Delta n}-\sum_{i=1}^{N} \rho_{\langle j\rangle} h_{i,\langle j\rangle}^{s} Y_{i,\langle j\rangle} V_{i,\langle j\rangle} \cdots\right. \\
\left.-q_{R,\langle j\rangle}-\mathcal{L}_{\langle j\rangle}+\sum_{i=1}^{N} w_{i,\langle j\rangle}^{\prime} h_{i,\langle j\rangle}^{c}+h_{s,\langle j\rangle} \sum_{i=1}^{N} w_{i,\langle j\rangle}^{\prime}-q_{c, s,\langle j\rangle}\right)^{k} .
\end{array}
$$

\subsubsection{Solving for Pressure and Its Interface Conditions}

The pressure at a boundary for low Mach number flows has been under scrutiny over the past thirty years. Gresho and Sani [54] give a thorough review of how an equation for pressure and its boundary conditions are implicitly derived for incompressible flows. In their review they show how the pressure Poisson equation (PPE) is obtained by taking the divergence of the momentum equation ${ }^{6}$

$$
\begin{aligned}
\nabla \cdot\left(\frac{\partial \rho \vec{v}}{\partial t}+\rho \vec{v} \cdot(\nabla \vec{v})\right. & =-\nabla p+\nabla \cdot \mathbb{T}) \\
\nabla \cdot \frac{\partial \rho \vec{v}}{\partial t}+\nabla \cdot \rho \vec{v} \cdot \nabla \vec{v} & =-\nabla^{2} p+\nabla \cdot \nabla \cdot \mathbb{T} \\
\nabla^{2} p & =\nabla \cdot \nabla \cdot \mathbb{T}-\nabla \cdot \frac{\partial \rho \vec{v}}{\partial t}-\nabla \cdot \rho \vec{v} \cdot \nabla \vec{v} .
\end{aligned}
$$

This derived equation for pressure is elliptic in nature, and thus requires information about pressure on all boundaries [55]. As noted by Ferziger and Peric [56] the PISO [39] method is, in essence, solving the PPE. Therefore, this work requires proper pressure boundary

\footnotetext{
${ }^{6}$ In Gresho and Sani's work they derive the PPE for incompressible flows, $\nabla \vec{v}=0$; however, density is still important in this derivation.
} 
conditions and a thorough analysis of how the conditions are specified.

Equation 2.39 is a derived, scalar, elliptic equation, which requires concomitant ${ }^{7}$ boundary conditions along the boundary of the simulated domain. These boundary conditions are derived by extracting the pressure gradient in the normal direction of the boundary,

$$
\begin{aligned}
\vec{n} \cdot\left(\frac{\partial \vec{\rho}}{\partial t}+\rho \vec{v} \cdot(\nabla \vec{v})\right. & =-\nabla p+\nabla \cdot \mathbb{T}) \\
\frac{\partial \rho v_{n}}{\partial t}+\rho \vec{v} \cdot\left(\nabla v_{n}\right) & =-\nabla_{n} p+\nabla_{n} \cdot \nabla \cdot \mathbb{T} \\
\frac{\partial p}{\partial n} & =-\frac{\partial \rho v_{n}}{\partial t}-\vec{v} \cdot\left(\nabla v_{n}\right)+\nabla_{n} \cdot \nabla \cdot \mathbb{T} .
\end{aligned}
$$

For illustrative purposes, consider Equation 2.40 in cartesian coordinates under the restriction of constant density and viscocity, $\mu$,

$$
\frac{\partial p}{\partial n}=-v_{x} \frac{\partial v_{n}}{\partial x}-v_{y} \frac{\partial v_{n}}{\partial y}-v_{z} \frac{\partial v_{n}}{\partial z}-\frac{\partial v_{n}}{\partial t}+\frac{\mu}{\rho}\left(\frac{\partial^{2} v_{n}}{\partial x^{2}}+\frac{\partial^{2} v_{n}}{\partial y^{2}}+\frac{\partial^{2} v_{n}}{\partial z^{2}}\right)
$$

For steady state, fully developed, top-hat, and far-field boundaries where velocity is specified, Equation 2.41 implies that ${ }^{8} \frac{\partial p}{\partial n}=0$, also referred to as zero-gradient. Gresho and Sani [54] state that using $\frac{\partial p}{\partial n}=0$ for cases where $v_{n} \neq 0$ along the boundary is "clearly wrong" due to remaning viscous terms (and possible unsteady velocity). Although their review contains detailed information on what conditions must be specified for pressure at a boundary, contradictory and confusing information has been published in the years since. These contradictions were recently addressed by Vreman [57] in work that specifically investigated no-slip walls with staggered grids. The contradictions were somewhat resolved by showing that the pressure gradient reaches an ambient, non-zero value for direct numerical simulation solutions; however, the question of what the proper pressure conditions are at an interface is on-going.

\footnotetext{
${ }^{7}$ naturally accompanying or associated

${ }^{8}$ Fixed velocity that doesn't abruptly change implies that $\nabla \cdot \vec{v}=\nabla^{2} \vec{v}=0$.
} 


\section{PISO Method}

The ambiguity of the boundary conditions for pressure is where the momentum predictor methods, such as the PISO method used in this work, were thought to be advantageous. Typical CFD books, such as Ferziger and Peric [56] as well as Hirsch [47], state that a fixed velocity boundary condition requires a zero-gradient condition for pressure, $\frac{\partial p}{\partial n}=0$, when using the PISO method. Although this is an easy boundary condition to implement, the declaration that pressure should be zero-gradient is wrong. The confusion of this boundary condition is illustrated in this section by exploring the PISO method's behavior at the boundary. Reconsider the FVM equation for scalar $\bar{\phi}$,

$$
A_{P} \bar{\Phi}_{i, P}^{n}+\sum_{L} A_{L} \bar{\Phi}_{i, L}^{n}=Q_{i}^{n-1} .
$$

The mometum equation implemented in OpenFOAM using Equation 2.42 is

$$
A_{P} \vec{v}^{n}+\sum_{L} A_{L} \vec{v}_{L}^{n}=Q^{n-1}-\nabla p^{n-1}
$$

Here the pressure gradient and source term (in OpenFOAM this is the viscous dissipation) are treated explicitly, $A_{L}$ are the coefficients due to FVM discretization of Laplacian and fluxes for neighboring cells, and $A_{P}$ is the coefficient of the point of interest. The superscript $n$ denotes the final value after solving the linear system. The source and pressure terms have an $n-1$ superscript because they are treated explicitly. Solving this system finds a $\vec{v}$ that satisfies the momentum equation but not the continuity equation. To provide closure, the PISO method uses some form of the PPE to assure the satisfaction of the continuity equation. However, because pressure and momentum are linked, an iterative process must be used where the objective is for the pressure to reach $n$ where $\vec{v}$ simultaneously satisfies the mometum and continuity equation. The PISO method, as originally written, is used to solve the coupled pressure-and velocity terms through the following procedures: 
1. Use the latest results for $v$ and $p$ as estimates for the predicted velocity field, $v^{n *}$.

$$
\vec{v}^{n *}=\frac{1}{A_{p}}\left(-\sum_{L} A_{L} \vec{v}_{L}^{n *}+Q^{n-1}-\nabla p^{n-1}\right)
$$

This predictor step enforces momentum but not continuity.

2. To enforce consistency between continuity and momentum equations, assume that pressure and velocity both need to be corrected by $p^{\prime}$ and $v^{\prime}$ respectively. This results in two expressions for the final pressure and velocity in terms of correctors, $p^{n}=$ $p^{n-1}+p^{\prime}$ and $\vec{v}^{n}=\vec{v}^{n *}+\vec{v}^{\prime}$. First, pressure and velocity corrections need to be linked. This is obtained through substituing $\vec{v}^{n}$ and $p^{n}$ as defined by their correctors into the momentum equation 2.44 ,

$$
\begin{aligned}
A_{P} \vec{v}^{n *}+\sum_{L} A_{L} \vec{v}_{L}^{n *}-Q^{n-1}+\nabla p^{n-1} & =0 \\
A_{P}\left(\vec{v}^{n}-\vec{v}^{\prime}\right)+\sum_{L} A_{L}\left(\vec{v}^{n}-\vec{v}^{\prime}\right)_{L}-Q^{n-1}+\nabla\left(p^{n}-p^{\prime}\right) & =0 \\
A_{P} \vec{v}^{n}-A_{P} \vec{v}^{\prime}+\sum_{L} A_{L} \vec{v}^{n}-\sum_{L} A_{L} \vec{v}_{L}^{\prime}-Q^{n-1}+\nabla p^{n}-\nabla p^{\prime} & =0 \\
\underbrace{A_{P} \vec{v}^{n}+\sum_{L} A_{L} \vec{v}^{n}-Q^{n-1}+\nabla p^{n}}_{\text {Continuity Equation }}-A_{P} \vec{v}^{\prime}-\sum_{L} A_{L} \vec{v}_{L}^{\prime}-\nabla p^{\prime} & =0 \\
\vec{v}^{\prime \prime}=\frac{-\sum_{L} A_{L} \vec{v}_{L}^{\prime}}{A_{P}}+\frac{-\nabla p^{\prime}}{A_{P}} &
\end{aligned}
$$

Equation 2.45 gives the velocity correction in terms of $p^{\prime}$. An expression for $p^{\prime}$ is 
obtained by constraining $v^{n}$ to the continuity equation, $\nabla \cdot \rho \vec{v}^{n}$,

$$
\begin{aligned}
\nabla \cdot \rho \vec{v}^{n} & =0 \\
\nabla \cdot \rho\left(\vec{v}^{n *}+\vec{v}^{\prime}\right) & =0 \\
\nabla \cdot \rho\left(\vec{v}^{n *}+\frac{-\sum_{L} A_{L} \vec{v}_{L}^{\prime}}{A_{P}}+\frac{-\nabla p^{\prime}}{A_{P}}\right) & =0 \\
\nabla \cdot\left(\frac{-\rho}{A_{P}} \nabla p^{\prime}\right)=\nabla \cdot \rho \vec{v}^{n *}-\nabla \cdot \frac{\rho \sum_{L} A_{L} \vec{v}_{L}^{\prime}}{A_{P}} . &
\end{aligned}
$$

Equations 2.45 and 2.46 link the velocity and pressure corrections together and a typical PISO iteration solves the two equations twice. Throughout the PISO method, the $\frac{\rho \sum_{L} A_{L} \vec{v}_{L}^{\prime}}{A_{P}}$ term is neglected, which is the main assumption of the PISO method. If the objective is to to reach $\vec{v}^{\prime} \rightarrow 0$, then this assumption is justified if the method reaches a stable solution [39]. In the second iteration, Equation 2.46 is used with the $\vec{v}^{\prime}$ terms to calculate a second pressure correction, $p^{\prime \prime}$. This second pressure correction is then used in Equation 2.45 to calculate the last velocity correction.

3. Repeat the previous steps until $p^{\prime}$ and $u^{\prime}$ are numerically small

Consider the application of the PISO method at a boundary with fixed velocity, $\vec{v}^{\prime} \cdot \vec{n}=$ $v_{n}^{\prime}=0$, which results in $\vec{v}_{n}^{n}=\vec{v}^{n *} \cdot \vec{n}=v_{n}^{n}=v_{n}^{n *}$, where $\vec{n}$ is the normal vector on the boundary. Knowing this, Equation 2.45 can be derived again, but with a rather useful 
constraint on the boundary,

$$
\begin{aligned}
& A_{P} \vec{v}^{n *}+\sum_{L} A_{L} \vec{v}_{L}^{n *}-Q^{n-1}+\nabla p^{n-1}=0, \\
& A_{P}\left(\vec{v}^{n}-\vec{v}^{\prime}\right)+\sum_{L} A_{L}\left(\vec{v}^{n}-\vec{v}^{\prime}\right)_{L}-Q^{n-1}+\nabla\left(p^{n}-p^{\prime}\right)=0 \\
& \vec{n} \cdot\left(A_{P} \vec{v}^{n}-A_{P} \vec{v}^{\prime}+\sum_{L} A_{L} \vec{v}^{n}-\sum_{L} A_{L} \vec{v}_{L}^{\prime}-Q^{n-1}+\nabla p^{n}-\nabla p^{\prime}\right)=0, \\
& \underbrace{A_{P} v_{n}^{n}+\sum_{L} A_{L} v_{n}^{n}-Q^{n-1}+\frac{\partial p^{n}}{\partial n}}_{\text {Continuity Equation }}-A_{P} v_{n}^{r^{0}}-\underbrace{\sum_{L} A_{L} y_{L, n}^{\prime}}_{\text {yssumed as } 0}-\frac{\partial p^{\prime}}{\partial n}=0, \\
& \frac{\partial p^{\prime}}{\partial n}=0 \text { on the boundary. }
\end{aligned}
$$

This goes on to imply that $v_{n}^{\prime}=0$ and $\frac{\partial p^{\prime}}{\partial n}=0$ on the second iteration. Using the definition for corrected pressure, $p^{n}=p^{n-1}+p^{\prime}$, it is concluded that $\frac{\partial p^{n}}{\partial n}=\frac{\partial p^{n-1}}{\partial n}$, which means that the pressure gradient must remain constant in all iterations. This does not mean that the gradient is zero for all iterations.

The confusion that led to the use of the wrong boundary conditions is that there are conflicting views on how the PISO method is implemented. If the PISO method is implmented so only $p^{\prime}$ is solved for, then one should set the boundary condition to zero-gradient. The solution procedure used in this disseration uses the PISO method that solves for $p$ instead of $p^{\prime}$ to correct the pressure within the domain. In the case where only $p^{\prime}$ is solved for, one only needs to establish reference pressures. This realization, however, is already known. Issa explains in his seminal work that established this method [39]:

When the velocity at the boundary is prescribed, all intermediate values of $v^{n *}$ at the boundary are set to the given boundary value and $\frac{\partial p^{\prime}}{\partial n}=0$...these serve as boundary conditions for the pressure-increment equations. ${ }^{9}$

\footnotetext{
${ }^{9}$ The notation has changed from Issa's notation to match this dissertation
} 
The implementation of the PISO method in the solver used in this dissertation is slightly different from the typical implementation due to the presence of density changes as well as the constant pressure assumption in the equation of state. Reconsider the finite volume discretisation of velocity:

$$
\vec{v}^{n *}=\frac{1}{A_{p}}\left(-\sum_{L} A_{L} \vec{v}_{L}^{n *}+Q^{n-1}-\nabla p^{n-1}\right)
$$

An equation for the hydrodynamic pressure is obtained by restricting $\vec{v}^{n *}$ to the continuity relation,

$$
\begin{aligned}
\frac{\partial \rho}{\partial t}+\nabla \cdot \rho \vec{v}^{n *}= & \frac{\partial \rho}{\partial t}+\nabla \cdot \frac{\rho}{A_{p}}\left(-\sum_{L} A_{L} \vec{v}_{L}^{n *}+Q^{n-1}-\nabla p^{n-1}\right)=0 \\
\frac{\partial \rho}{\partial t}+\nabla \cdot \frac{\rho}{A_{p}}\left(-\sum_{L} A_{L} \vec{v}_{L}^{n *}+Q^{n-1}-\nabla p^{n-1}\right) & =0 .
\end{aligned}
$$

Equation 2.48 is rearranged to solve for $p^{n}$ with the additional adjustment that $\rho=\psi p_{o}$, where $\psi$ is the compressibility factor,

$$
\begin{aligned}
& \nabla \cdot \frac{\rho}{A_{p}} \nabla p^{n}=\frac{\partial \psi p_{o}}{\partial t}+\nabla \cdot \frac{\rho}{A_{p}}\left(-\sum_{L} A_{L} \vec{v}_{L}^{n *}+Q^{n-1}\right) \\
& \nabla \cdot \frac{\rho}{A_{p}} \nabla p^{n}=\frac{\partial \psi}{\partial t} p_{o}+\nabla \cdot \frac{\rho}{A_{p}}\left(-\sum_{L} A_{L} \vec{v}_{L}^{n *}+Q^{n-1}\right)
\end{aligned}
$$

Equation 2.48 differs from Equation 2.46 in that $p^{n}$ is solved for instead of $p^{\prime}$. The rest of the implementation is the same; fluxes and velocities are updated due to the pressure equation prediction and the steps are iterated twice. The main thing to note here is that $p^{n}$ is solved for and thus the derived boundary condition for pressure is required. This boundary condition is found by accessing the normal component of the steady state momentum 
equation,

$$
\begin{aligned}
\vec{n} \cdot\left(\frac{\partial \rho \vec{v}}{\partial t}+\nabla \cdot(\rho \vec{v} \vec{v})\right) & =\vec{n} \cdot(-\nabla p+\nabla \cdot \mathbb{T}), \\
\rho \frac{\partial v_{n}}{\partial t}+\rho \vec{v} \cdot \nabla v_{n} & =-\frac{\partial p}{\partial n}+\vec{n} \cdot \nabla \cdot \mathbb{T} \\
\frac{\partial p}{\partial n} & =\vec{n} \cdot \nabla \cdot \mathbb{T}-\rho \frac{\partial y_{n}^{\prime}}{\partial t}-\rho \vec{v} \cdot \nabla v_{n} \\
\frac{\partial p}{\partial n} & =\vec{n} \cdot \nabla \cdot \mathbb{T}-\rho \vec{v} \cdot \nabla v_{n} .
\end{aligned}
$$

Such an expression can easily be solved for between iterations using OpenFOAM's finite volume calculus operators.

\subsubsection{Summary of Interface Conditions}

The interface conditions in terms of partial differential equations are listed in Table 2.2

Table 2.2: Conservation equations at a reacting carbon interface

\begin{tabular}{|c|c|}
\hline Velocity & $v_{n}=\frac{\sum_{i=1}^{N} w_{i}^{\prime}}{\rho}$ \\
\hline Pressure & $\frac{\partial p}{\partial n}=\vec{n} \cdot \nabla \cdot \mathbb{T}-\rho \vec{v} \cdot \nabla v_{n}$ \\
\hline Species & $\frac{\partial}{\partial t}\left(\rho^{\prime} Y_{i}\right)+\rho Y_{i} v_{n}-\rho D_{i} \frac{\partial Y_{i}}{\partial n}-w_{i}^{\prime}=0$ \\
\hline Enthalpy & $\frac{\partial}{\partial t}\left(\rho^{\prime} h^{s}\right)+h^{s} \rho v_{n}-\frac{\lambda_{g}}{c_{p, g}} \frac{\partial h^{s}}{\partial n}+\sum_{i=1}^{N} \rho_{g} h_{i}^{s} Y_{i} V_{i}+q_{R}$ \\
& $-\mathcal{L}-\sum_{i=1}^{N} w_{i}^{\prime} h_{i}^{c}=h_{s} \sum_{i=1}^{N} w_{i}^{\prime}-\frac{\lambda_{s}}{c_{p, s}} \frac{\partial h_{s}^{s}}{\partial n}$ \\
\hline
\end{tabular}




\subsection{Heterogeneous Reaction Models}

Presented here are the heterogeneous reaction rate parameters used to calculate the mass production source term, $w_{i}^{\prime}$, for the species conservation at a reacting interface.

\subsubsection{Nonporous Carbon}

The specific reaction rate parameters used to model graphite carbon surface reactions are listed in Table 2.3.

\begin{tabular}{llllc}
\hline Reaction & $i$ & $B_{i}$ & $n_{i}$ & $E_{i}$ \\
\hline$C_{s}+\mathrm{OH} \rightarrow \mathrm{CO}+\mathrm{H}$ & 1 & $3.61 \times 10^{2}$ & -0.5 & 0 \\
$C_{s}+\mathrm{O} \rightarrow \mathrm{CO}$ & 2 & $6.65 \times 10^{2}$ & -0.5 & 0 \\
$C_{s}+\mathrm{H}_{2} \mathrm{O} \rightarrow \mathrm{CO}+\mathrm{H}_{2}$ & 3 & $4.80 \times 10^{5}$ & 0.0 & 68800 \\
$C_{s}+\mathrm{CO}_{2} \rightarrow 2 \mathrm{CO}$ & 4 & $9.00 \times 10^{3}$ & 0.0 & 68100 \\
$C_{s}+\frac{1}{2} \mathrm{O}_{2} \rightarrow \mathrm{CO}$ & 5 & $2.40 \times 10^{3}$ & 0.0 & 30000 \\
& 6 & $2.13 \times 10^{1}$ & 0.0 & -4100 \\
& 7 & $5.35 \times 10^{-1}$ & 0.0 & 15200 \\
& 8 & $1.81 \times 10^{7}$ & 0.0 & 97000 \\
\hline
\end{tabular}

Table 2.3: The heterogeneous reaction rate parameters of non-porous carbon as compiled by Bradley et al. [24].

Knowing the specific reaction rate parameters listed in Table 2.3, the mass production rate ( $w_{i}^{\prime}$ is in units of $\mathrm{kg} / \mathrm{m}^{2} / \mathrm{s}$ ) can be calculated by using the modified-Arrhenius expression,

$$
k_{i}=B_{i} T^{n_{i}} \exp \left(-E_{i} / R T\right),
$$


with the following surface mass production rate relations:

$$
\begin{aligned}
& w_{O H}^{\prime}=-\left(p_{O H}\right) k_{1} \\
& w_{O}^{\prime}=-\left(p_{O}\right) k_{2} \\
& w_{H_{2} O}^{\prime}=-\left(p_{H_{2} O}\right)^{\frac{1}{2}} k_{3} \\
& w_{C O_{2}}^{\prime}=-\left(p_{C O_{2}}\right)^{\frac{1}{2}} k_{4} \\
& Y=\frac{1}{1+\frac{k_{8}}{k_{7} p_{2}}} \\
& w_{O_{2}}^{\prime}=\left(-\frac{1}{2}\right)\left[\frac{k_{5} p_{O_{2}} Y}{1+k_{6} p_{O_{2}}}+k_{7} p_{O_{2}}\left(1-Y^{\prime}\right)\right] \\
& w_{H}^{\prime}=\left(p_{O H}\right) k_{1} \frac{W_{H}}{W_{O H}}, \\
& w_{H_{2}}^{\prime}=\left(p_{H_{2} O}\right) k_{3} \frac{W_{H_{2}}}{W_{H_{2} O}}, \\
& w_{C O}^{\prime}=-\left(w_{O H}^{\prime} \frac{W_{C O}}{W_{O H}}+w_{O}^{\prime} \frac{W_{C O}}{W_{O}}+\frac{w_{H_{2} O}^{\prime}}{W_{H_{2} O}}+w_{C O_{2}}^{\prime} \frac{2 W_{C O}}{W_{C O_{2}}}+w_{O_{2}}^{\prime} \frac{2 W_{C O}}{W_{O_{2}}}\right)
\end{aligned}
$$

Here, $p_{i}$ denotes partial pressure of species $i$ in atmospheric pressure units.

\subsubsection{Porous Model}

The reaction rate parameters for porous carbon surfaces are listed in Table 2.4.

\begin{tabular}{lllll}
\hline$k$ & Reaction & $B_{k}$ & $n_{k}$ & $E_{k}$ \\
\hline 1 & $C_{s}+O H \rightarrow C O+H$ & 1.65 & 0.5 & 0 \\
2 & $C_{s}+O \rightarrow C O$ & 3.41 & 0.5 & 0 \\
3 & $C_{s}+H_{2} O \rightarrow C O+H_{2}$ & $2.00 \times 10^{8}$ & 0.0 & 64750 \\
4 & $C_{s}+\mathrm{CO}_{2} \rightarrow 2 \mathrm{CO}$ & $6.00 \times 10^{7}$ & 0.0 & 64300 \\
5 & $C_{s}+\frac{1}{2} \mathrm{O}_{2} \rightarrow \mathrm{CO}$ & $4.40 \times 10^{6}$ & 0.0 & 43000 \\
\hline
\end{tabular}

Table 2.4: The heterogeneous rate constants of porous carbon [25] 
Here, the mass production rate is calculated using the expression,

$$
w_{i}^{\prime}=\sum_{k=1}^{5} \nu_{i k} W_{i}\left(\rho Y_{k} / W_{k}\right) B_{k} T^{n_{k}} \exp \left(-E_{k} / R T\right),
$$

where subscript $i$ denotes the species of interest, while subscript $k$ denotes the particular reaction step in Table 2.4. Specifically, $W_{i}$ is the molecular weight of species $i, W_{k}$ and $Y_{k}$ are the molecular weight and mass fractions of the reactant species in reaction $k$, respectively, and $\nu_{i k}$ is the stoichiometric coefficient of species $i$ in the $k$ th reaction. The units of $w_{i}^{\prime}$ are $\mathrm{kg} / \mathrm{m}^{2} / \mathrm{s}$.

\subsubsection{Comparison of Heterogeneous Models}

Two sets of heterogeneous semi-global reaction models were used for the carbon surface reactions. The models include reactions with gaseous species $\mathrm{O}_{2}, \mathrm{CO}_{2}, \mathrm{H}_{2} \mathrm{O}, \mathrm{OH}$, and $\mathrm{O}$ atoms at the surface. These species react with the carbon surface, $\mathrm{C}_{s}$, and produce $\mathrm{CO}$ as well as traces of $\mathrm{H}_{2}$ and $\mathrm{H}$-atoms. The model compiled by Bradley et al. [24] is meant for pure graphite surfaces (assumed non-porous). The other model was developed by Chelliah et. al. [25] by tuning the Bradley et al. model to include 25 percent bulk porosity of the graphite surface. This was done by numerically adjusting the $\mathrm{C}_{s}+\mathrm{O}_{2}$ and $\mathrm{C}_{s}+\mathrm{CO}_{2}$ reactions to fit experimental results of a porous carbon surface.

Figure 2.6 shows the calculated ratio of the porous to nonporous models' reaction rates as a function of temperature for the tuned reactant species, $\mathrm{O}_{2}, \mathrm{CO}_{2}$, and $\mathrm{H}_{2} \mathrm{O}$. 


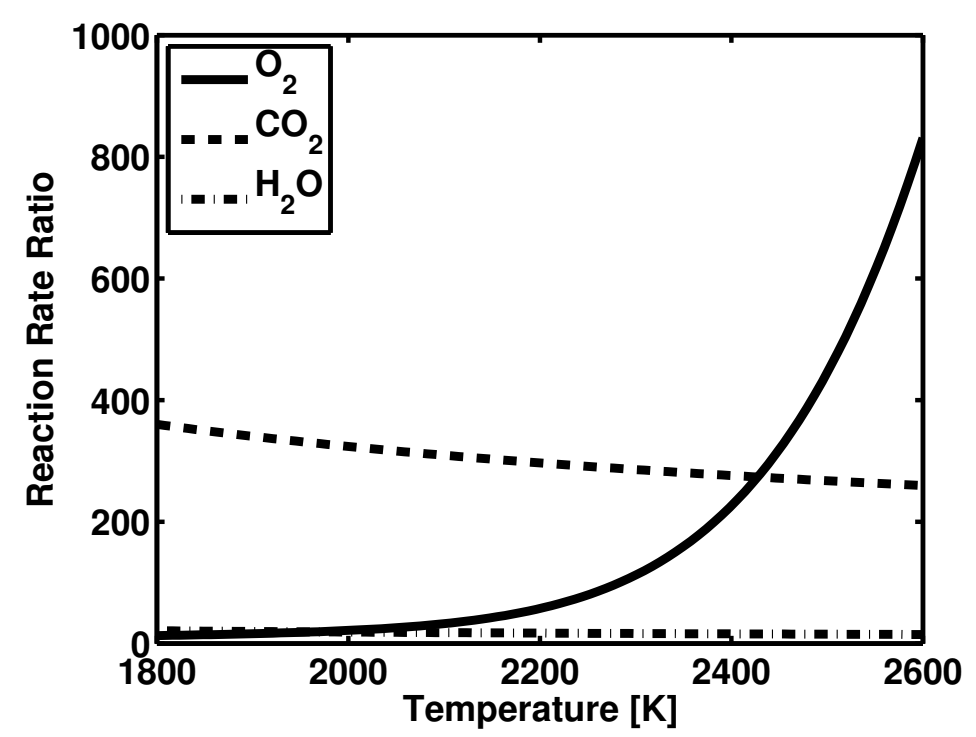

Figure 2.6: Comparisons of reaction rate ratio of porous reaction rates to nonporous reaction rates for $\mathrm{O}_{2}, \mathrm{CO}_{2}$, and water for the same composition and pressure.

These reaction rates were calculated using constant reference quantities for density, $\rho=$ $0.15 \mathrm{~kg} / \mathrm{m}^{3}$, and pressure, $p=1.0 \mathrm{~atm}$. According to these models, the porous carbon surface reaction rates for $\mathrm{O}_{2}$ and $\mathrm{CO}_{2}$ can be two orders of magnitude greater than the nonporous surfaces. At temperatures larger than $2200 \mathrm{~K}$, the $\mathrm{O}_{2}$ reaction rates for the porous model suddenly increases. This is due to the annealing phenomenon of carbon oxidation that is accounted for in the Bradley et al. model and causes the nonporous $\mathrm{O}_{2}$ reaction rates to relax at higher temperatures. Such annealing was not found to exist for porous reacting carbon surfaces in the Chelliah et al. work that developed the porous model.

\subsection{Model Verification}

The finite volume method code required several model verification tests. Each subroutine that calculated transport and thermodynamic properties was extensively tested and found to be consistent with CHEMKIN's transport subroutines. Additionally, several canonical combustion cases were simulated and gave confidence that the developed solver is consistent with the current state-of-the-art combustion codes. The sections that follow cover these 
verification tests in detail.

\subsubsection{Chemistry}

A direct comparison study was performed in order to verify the correct time integration of chemical source terms by the OpenFOAM solver. The ignition of a homogeneous mixture at constant pressure was simulated with initial mole fraction concentrations of 17 percent hydrogen, 17 percent oxygen, and 66 percent nitrogen. This homogeneous combustion case was verified against SENKIN's chemistry solver, and the results are shown in Figure 2.7.

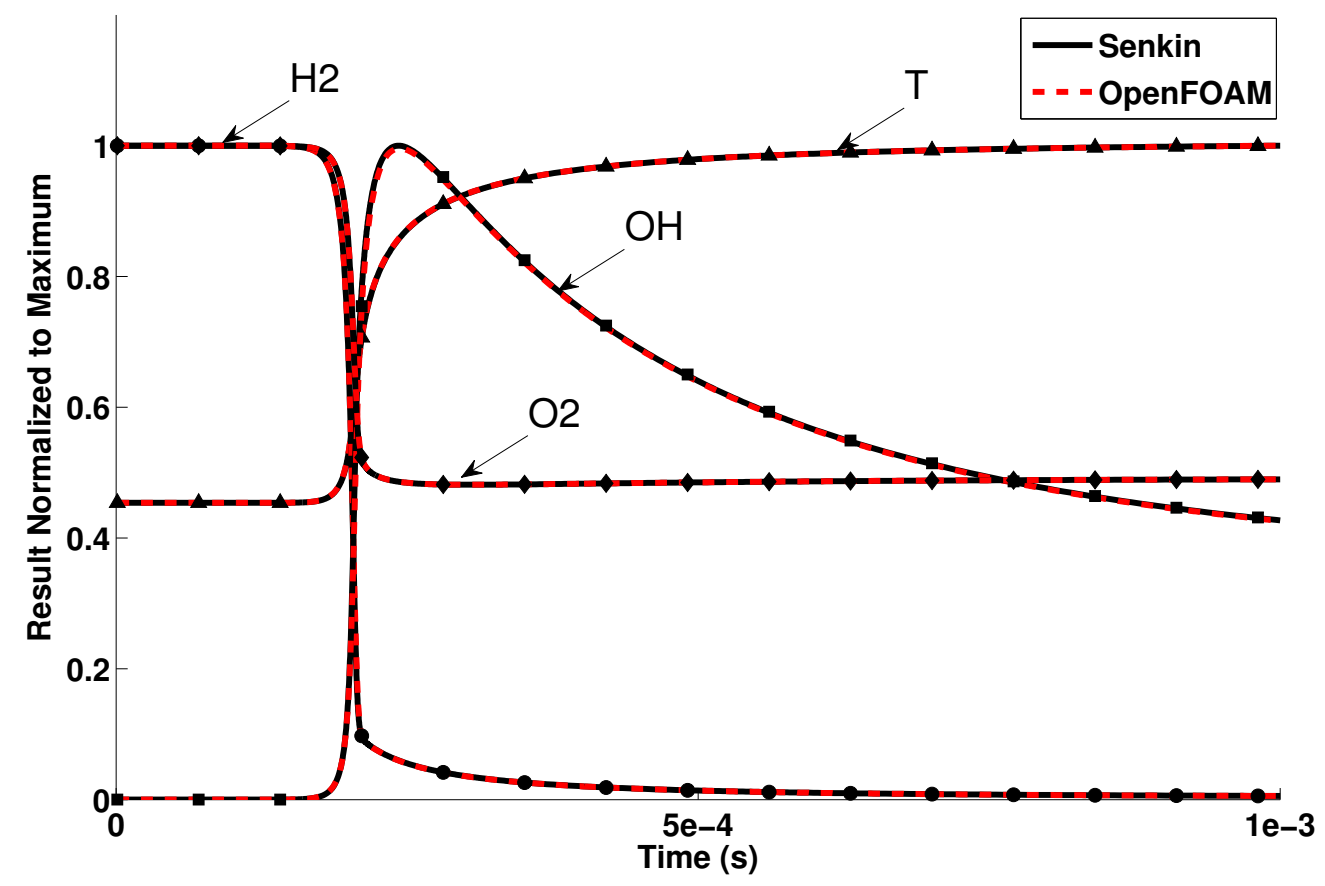

Figure 2.7: Homogeneous combusiton at constant temperature for both OpenFOAM and SENKIN codes. Parameters are normalized to thier maximum values, ie. $T / T_{\text {max }}$, $X_{H 2} /\left(X_{H 2}\right)_{\max }, X_{O 2} /\left(X_{O 2}\right)_{\max }, X_{O H} /\left(X_{O H}\right)_{\max }$

This was repeated for several different homogeneous mixture concentrations with the results showing the same consistency. 


\subsubsection{Premixed Flame}

A direct comparison study was performed between the OpenFOAM solver and Sandia's one-dimensional premixed code. A one-dimensional mesh was constructed for the OpenFOAM case, with grid spacing in the axial direction of $10 \mu \mathrm{m}$. A constant temperature profile of $980 \mathrm{~K}$ was specified in both the Sandia and OpenFOAM codes. The upstream boundary conditions were $2 \mathrm{~m} / \mathrm{s}$ for velocity, 0.004 for hydrogen molefraction, and 0.001 for oxygen mole fraction. Pressure was treated as a constant 1 atmosphere in the Sandia code. The OpenFOAM's PISO method found changes on the order of $10^{-} 3 \mathrm{~Pa}$ to pressure throughout the domain. It is important to note that Sandia's premixed code treats diffusion as upwind unless otherwise specified and OpenFOAM uses flux limiting schemes which combines both upwind and central differencing techniques. Therefore, the premixed flame was also simulated using a central differencing scheme in the Sandia code to better interpret the numerical effects of finite differencing. Figure 2.8 shows the mole fraction solutions for both the OpenFOAM and Sandia code results. 


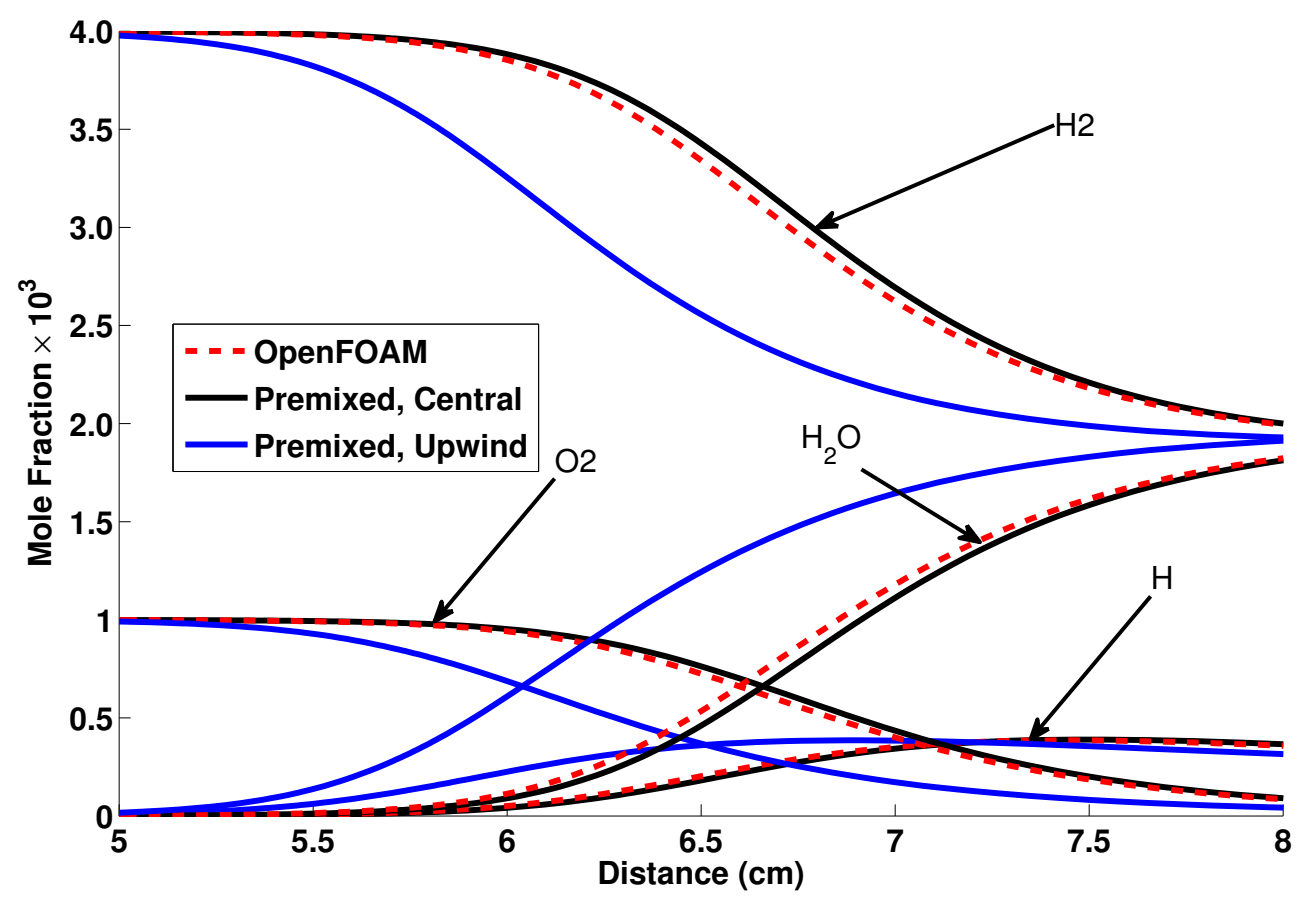

Figure 2.8: Spatial variation of premixed flame mole fraction concentrations for $\mathrm{H} 2, \mathrm{O} 2$, and $\mathrm{H} 2 \mathrm{O}$

The Sandia results shift according to the numerical scheme and OpenFOAM's results agree well with the more accuracte central differencing scheme solution.

\subsubsection{Counterflow Flame}

To verify that the OpenFOAM solver could sufficiently simulate counterflow flames, a direct comparison study was performed. The OpenFOAM domain considered was a $1.5 \mathrm{~cm}$ by $1.5 \mathrm{~cm}$ axisymmetric mesh and results were compared to Smooke's [36] quasi onedimensional code. The flames simulated were a diluted non-premixed hydrogen (mole fraction, $\left.X_{H_{2}}=0.16\right)$ vs. air $\left(X_{O_{2}}=0.21\right)$ flame. The OpenFOAM solutions were generated by prescribing uniform jet, plug-flow, velocity profiles at both hydrogen and air nozzle boundary conditions. The outflow boundary condition used a far-field condition for pressure and Neumann condition for all other quantities. The opposed stream flow velocities were gradually increased and convergence was confirmed at strain rates ranging from 200 
to $450 \mathrm{~s}^{-1}$ (quasi one-dimensional solutions predict flame extinction at $480 \mathrm{~s}^{-1}$ ). For consistency, both simulations used mixture-averaged diffusion for species transport, Burke et al.'s detailed kinetic model for hydrogen oxidation [58], and the same grid resolution of 15 $\mu \mathrm{m}$. Temperature profiles for two of these counterflow flames are shown in Figure 2.9 for the OpenFOAM and quasi one-dimensional converged solutions.

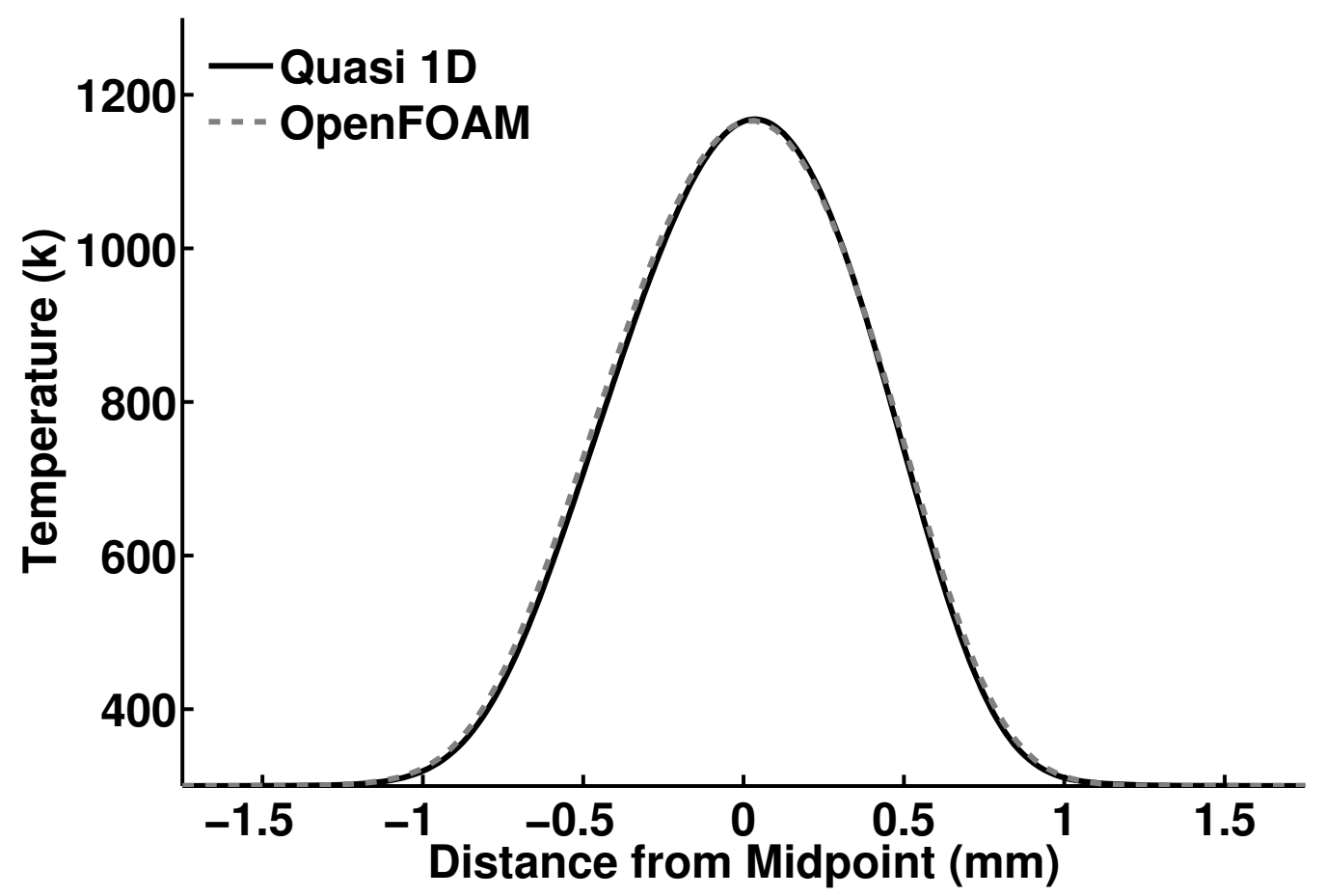

Figure 2.9: Counterflow flame temperature profiles for OpenFOAM and quasi onedimensional codes; for $a=420 \mathrm{~s}^{-1}$.

The flame temperature profiles for both OpenFOAM and quasi one-dimensional simulations are within $2 \mathrm{~K}$ agreement.

\subsubsection{Reacting Stagnation Plate Flow Configuration}

A verification test was performed using Smooke's quasi one-dimensional code [36] with a reacting carbon surface boundary condition. The results were compared to carbon mass loss rate data taken in 1996 by Makino et al. using the porous model [25]. The quasi one- 
dimensional solver matched strain-rate and free stream species concentration conditions. Figure 2.10a shows this validation test. Additionally, Figure 2.10b shows a comparison between OpenFOAM and the quasi one-dimensional solver with matched inflow velocities and free stream conditions and different surface temperatures (OpenFOAM inflow was plug-flow velocity profile). 


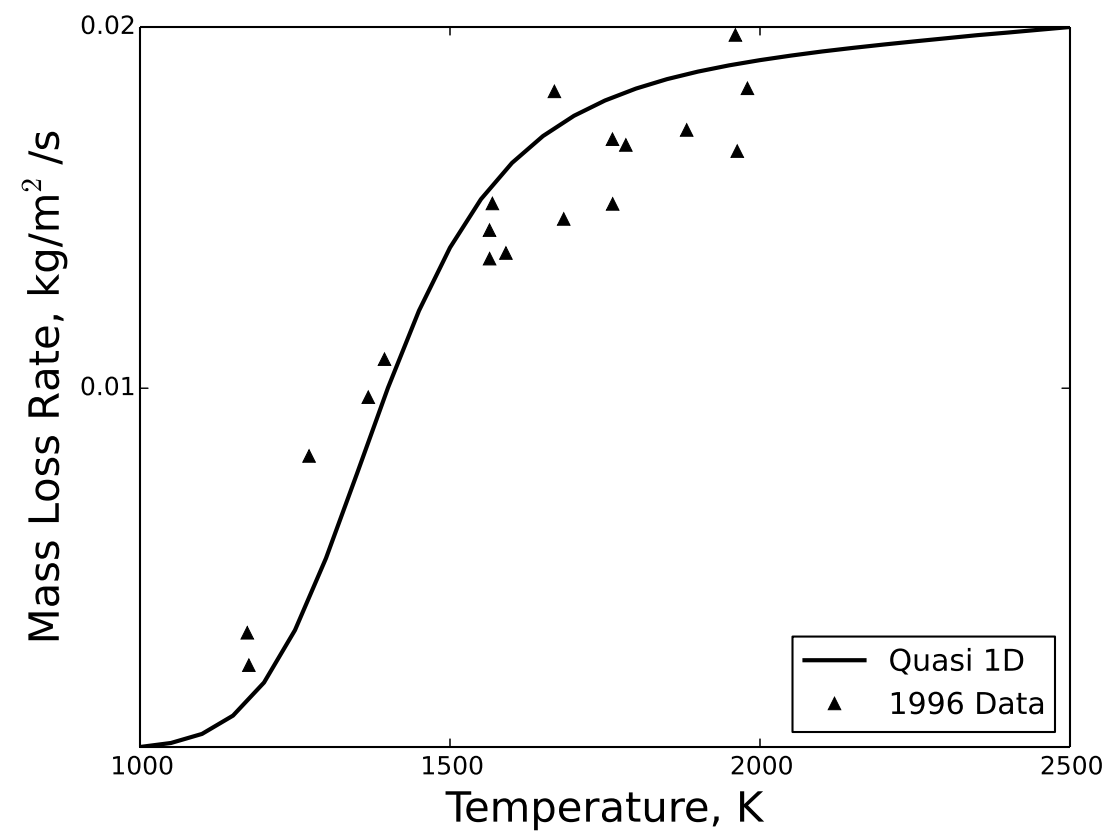

(a) Validation of quasi one-dimensional solver (data from Makino et al. [25])

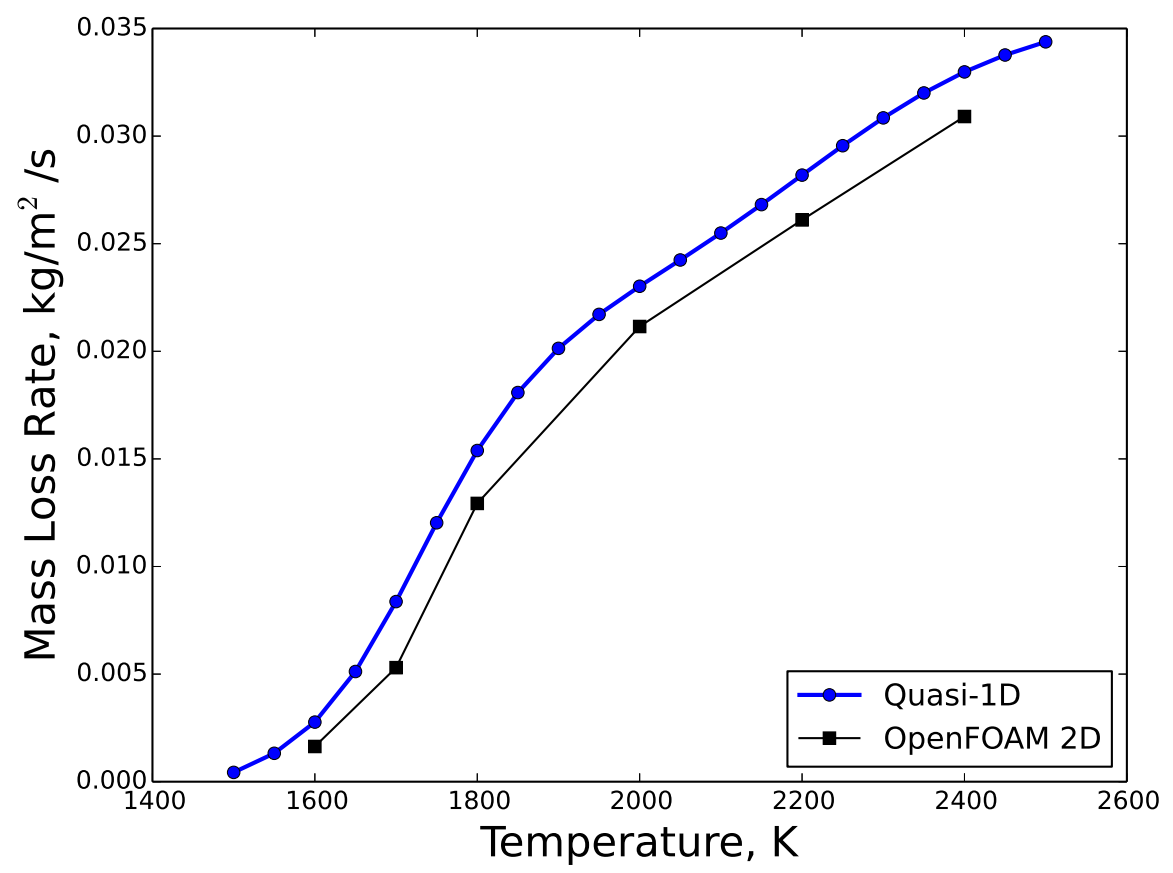

(b) Verification of OpenFOAM solver

Figure 2.10: Verification and validation tests of quasi one-dimensional and OpenFOAM FVM codes.

The data from Makino's 1996 mass loss rate experiments are in close agreement with the 
quasi one-dimensional simulations, serving as validation for the porous model and stagnation flow solver. The OpenFOAM and quasi one-dimensional solvers are also in strong agreement, and comparisons of the species profiles of these two codes are shown in 2.11a and $2.11 \mathrm{~b}$ for $\mathrm{CO}$ and $\mathrm{CO}_{2}$ respectively.

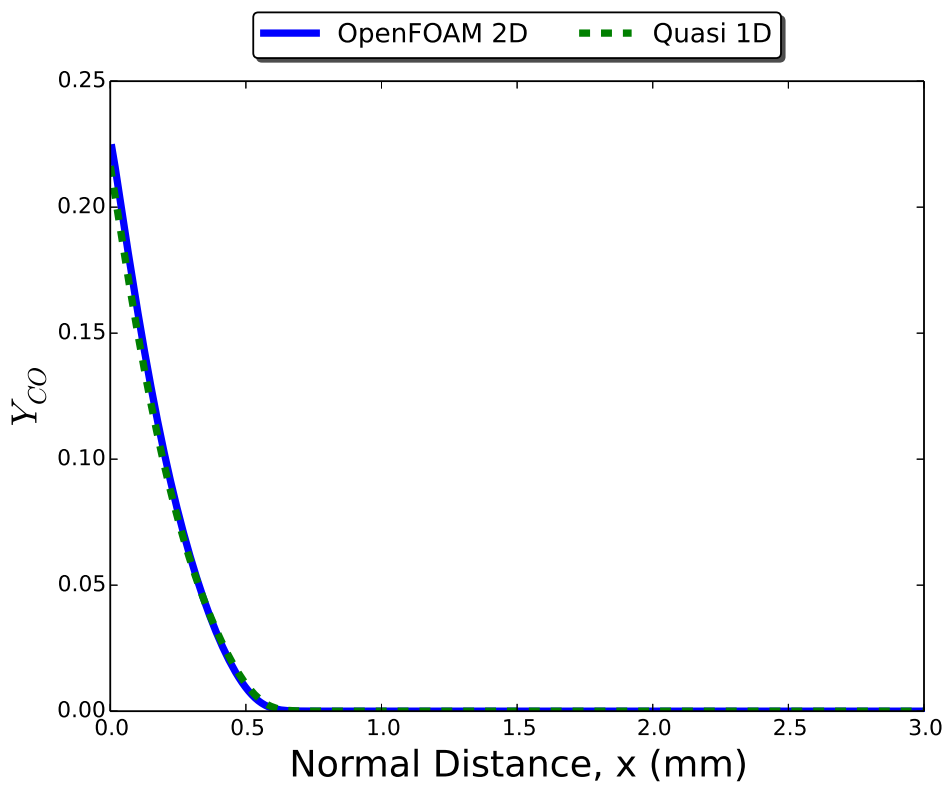

(a) $\mathrm{CO}$ mass fraction profile in normal direction

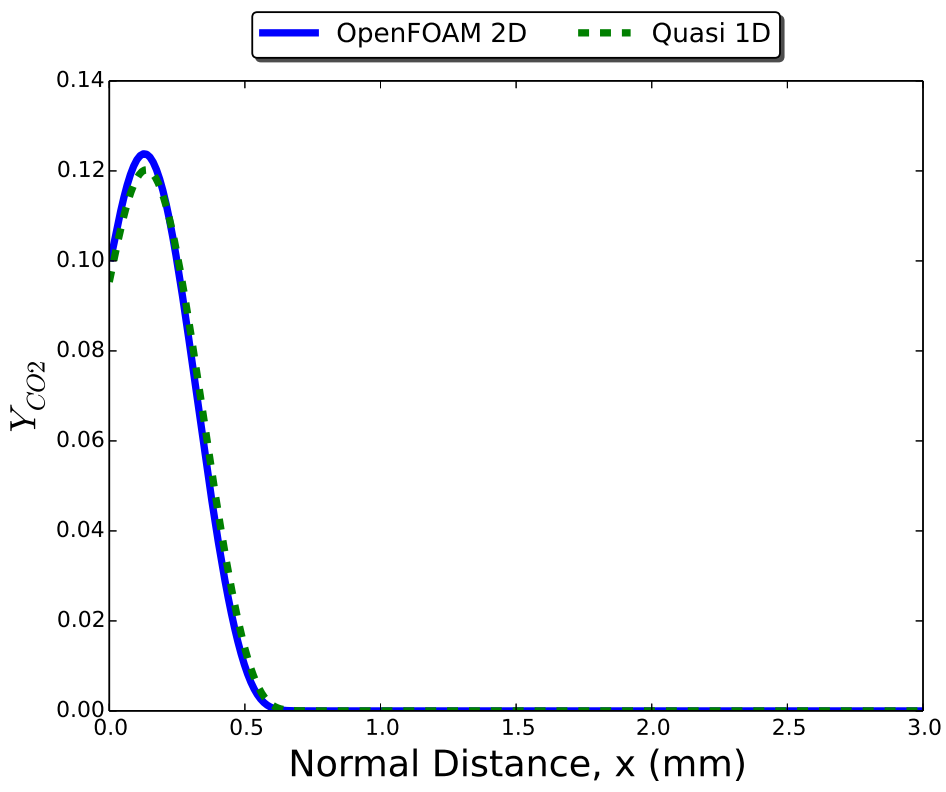

(b) $\mathrm{CO}_{2}$ mass fraction profile in normal direction

Figure 2.11: Reacting layer profile comparion 


\subsubsection{Grid Resolution Index}

Numerical error and grid dependence in this study were assessed in order to remove the possibility that the general trends highlighted are due to numerical sources. A systematic grid refinement study was performed to determine the grid independence for the OpenFOAM cases. The discretization scheme used here was second-order accurate, which dictates that the observed order of accuracy for a response quantity should be close to 2.0. Three consecutive grid refinements yielded observed order of accuracies of 1.84, 1.78, and 1.67 for strain rate, maximum temperature, and hydrogen atom concentrations for a reacting case, respectively. This confirms that the grid choices used are within the asymptotic range and grid independent. These observed orders of accuracy were greater than 1.5, which allowed a factor of safety of 1.5 in the calculation of a grid convergence index (GCI) using Roache's method and Richardson extrapolation (see Roy and Oberkamph [59]). This resulted in error estimates of less than $5 \%$ for $\Delta x=15 \mu \mathrm{m}$ for the peak temperature in the counterflow flame simulations that were used to verify the OpenFOAM solver. Figure 2.12 shows the strain rate curve for the cases simulated in Section 2.6.3.

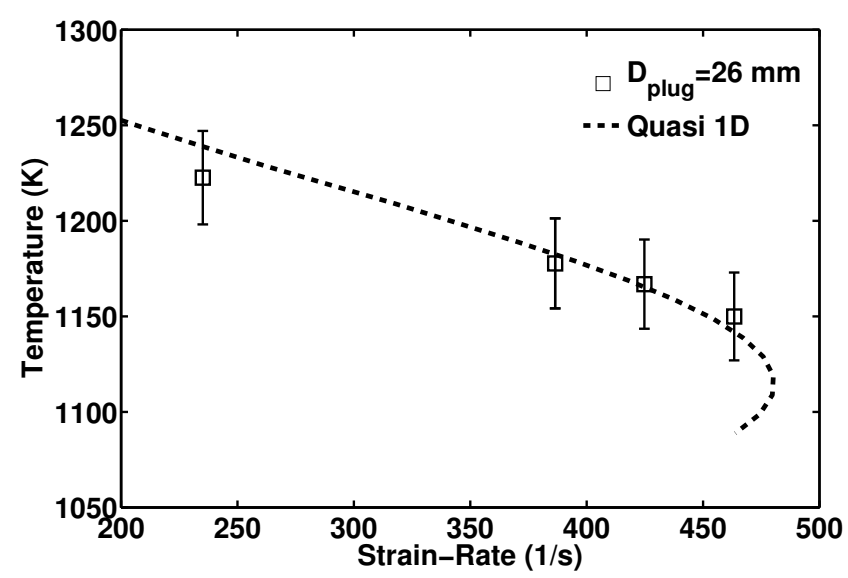

Figure 2.12: Decrease of maximum flame temperature with increasing strain rate for diluted non-premixed hydrogen vs. air, from both OpenFOAM and quasi one-dimensional simulations.

The cases were run with grid seperartion of $\Delta x=15 \mu \mathrm{m}$ which permits applying the $5 \%$ error to each OpenFOAM peak temperature. The quasi one-dimensional strain curve is 
within the numerical error of all the OpenFOAM solutions for plug flow inflow conditions. In addition to the OpenFOAM solver's numerical error, it was also necessary to analyze the quasi one-dimensional numerical effects on solutions. Such numerical effects are rarely discussed, as it is assumed that the quasi one-dimensional code results are mesh independent. This is due to the fact that both OppDiff [35] and Smooke's [36] codes use local grid refinements in regions of large gradients. Refinements are done without a noticeable computational cost and typically result in $5 \mu \mathrm{m}$ grid spacings (which is not computationally feasible for all CFD). However, it was found in this study that a quasi one-dimensional code's temperature solution can change by $10 \mathrm{~K}$ through a relaxation or stiffening of the aforementioned gradient refinement properties while retaining fine resolutions on the order of $10 \mu \mathrm{m}$.

In addition to the numerical sensitivity of the mesh refinement, the numerical schemes for calculating diffusion fluxes were investigated and resulted in an even larger effect on flame temperature profile. Figure 2.13 depicts the effect of changing the numerical scheme to calculate the species specific convective terms in the conservation equation from upwind to central differencing.

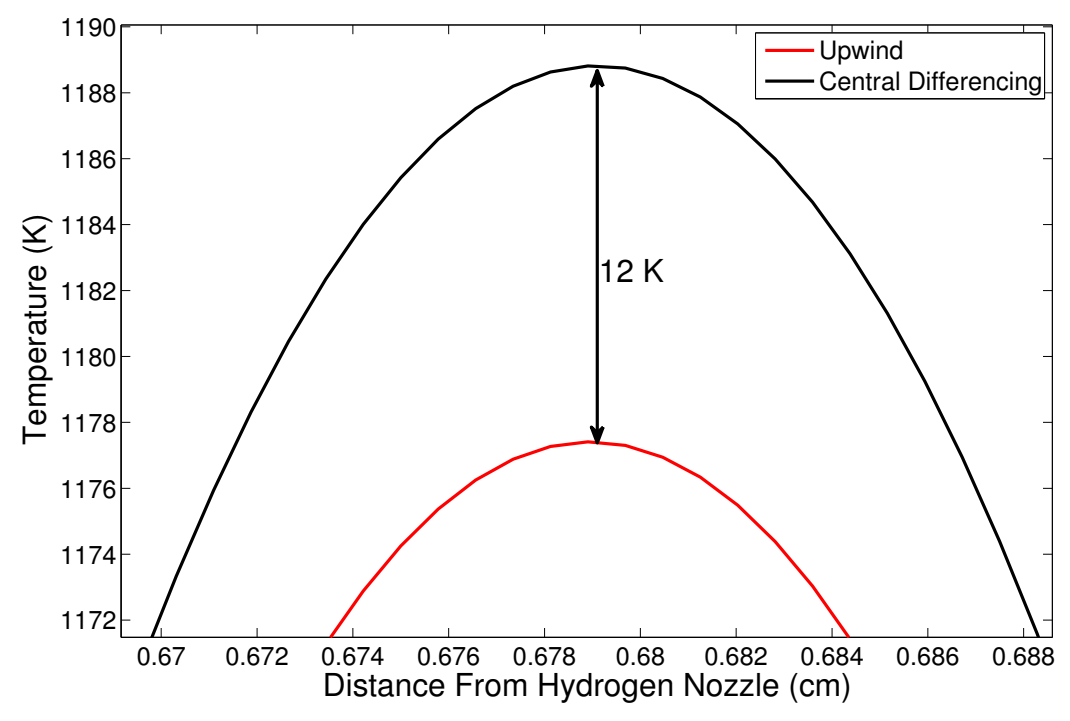

Figure 2.13: Decrease in peak temperature due to numerical scheme 
A change in diffusion flux calculations changes the temperature profile by $12 \mathrm{~K}$. These changes have little effect on momentum properties (e.g. strain rate), but they are important as this work considers one-to-one comparisons between OpenFOAM and the quasi onedimensional formulation. Therefore, this work did not consider a difference of less than 20 $\mathrm{K}$ (this difference combined with OpenFOAM's uncertainty) as physical, as the numerical effects cannot be removed as a possibility for the discrepancy. 


\section{Chapter 3}

\section{Flow Over a Reaction Carbon Plate}

\subsection{Background}

To date, Shulze et al. [30] is the only group that investigated the multidimensionality of the reacting layers of carbon surfaces in an air environment. Their group studied flow over a moving reacting carbon sphere with diameter $2 \mathrm{~mm}$ and concluded that flow parameters and surface temperatures affect the reacting layers over the sphere. This lack of research addressing the multidimensionality of coupled heterogeneous and homogeneous reactions for carbon surfaces is the main motivaiton of the work presented here. Specifically, there is no work that concerns this multidimensionality over a flat plate, which is one of the most common fluid dynamic geometries. To capture multidimensionality, carbon surface reaction models were implemented into a reacting flow solver using that uses the OpenFOAM computational package [60]. In order to analyze the effects of the coupled gas and surface kinetic rates and fluid residence time, the surface temperatures ranged from 1800 to $2600 \mathrm{~K}$ and free stream velocities ranged from 30 to $50 \mathrm{~m} / \mathrm{s}$. The simulated domain was $10 \mathrm{~mm}$ in length and $1 \mathrm{~mm}$ in height with a $5 \mathrm{~mm}$ long reacting carbon surface. 


\subsection{Computational Domain and Test Simulations}

An illustration of the two-dimensional computational domain is shown in Figure 3.1. The lengths of the computational domain are 10 and $1 \mathrm{~mm}$ in directions parallel and normal to the surface, respectively. Numeric labels are used to identify each separate boundary condition. The upstream (left) boundary, labeled as 1, is where the free-stream values for velocity, oxidizer composition, and temperature are specified as fixed value conditions. The top and downstream (right) boundaries, labeled as 2 and 3 respectively, specify pressure as a far-field boundary condition and all remaining variables are specified as zero-gradients. A portion of the lower boundary, labeled as 4 , is an $8 \mathrm{~mm}$ long flat plate that contains the reacting carbon surface. This boundary specifies viscous non-slip velocity and specified surface temperature, $T_{s}$. Within boundary 4 is the embedded reacting carbon surface of $5 \mathrm{~mm}$ length and is shown in red. The open section of the lower boundary, labeled as 5 , is a $1 \mathrm{~mm}$ long boundary that allows for the development of the flow field prior to the leading edge. The specific values assigned for all the physical boundary conditions are listed in Table 3.1. Not listed in this table are the value for free-stream velocity, $U_{\infty}$, and surface temperature, $T_{s}$, which are the variable input parameters in this investigation. The free-stream composition is specified as $Y_{\mathrm{O}_{2}}=0.21, Y_{\mathrm{H}_{2} \mathrm{O}}=0.02$ and $Y_{\mathrm{N}_{2}}=0.77$ corresponding to moist-air with relative humidity of $80 \%$. Excluding the reacting surface where concentrations are calculated as detailed in section 2.4 , the species boundary conditions are set to zero-gradient. 


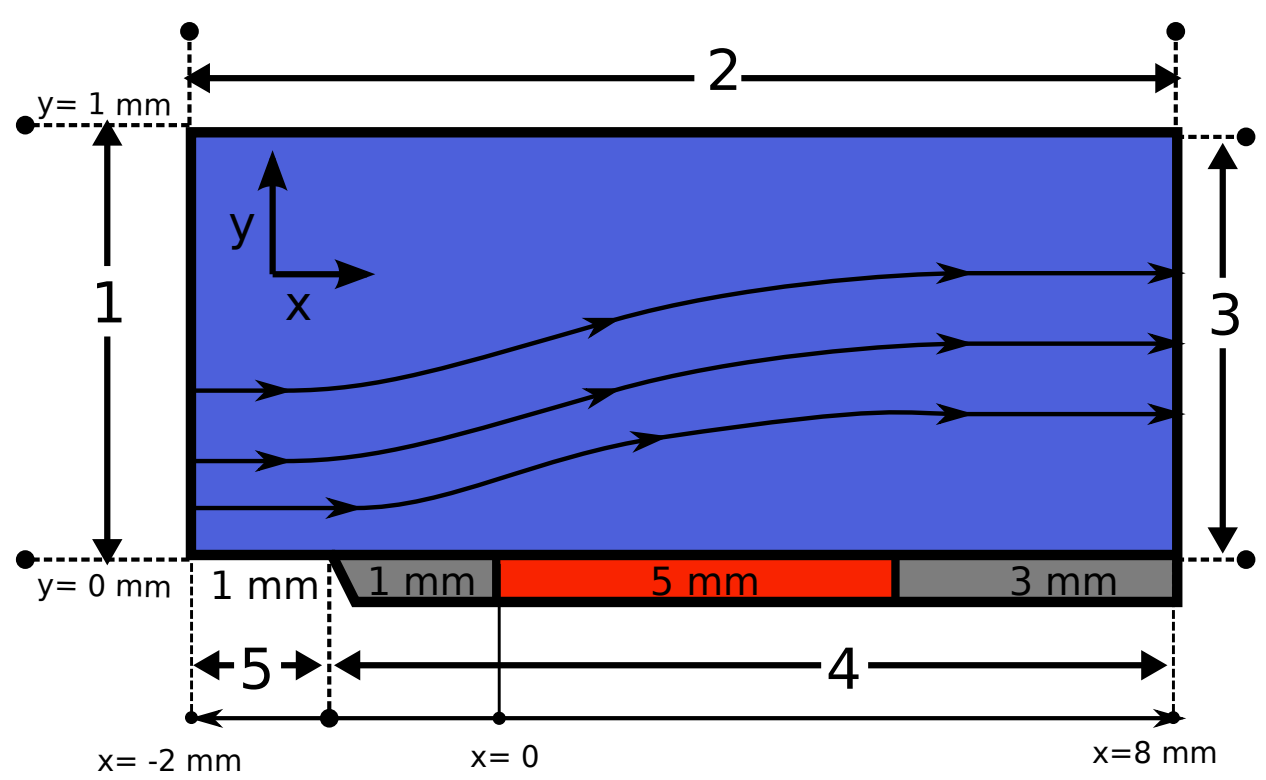

Figure 3.1: Two-dimensional computational domain used in present simulations; red area shows the reacting carbon sample is located.

Table 3.1: Numerical boundary conditions imposed on five surfaces identified in Fig. 3.1

\begin{tabular}{cccc}
\hline Boundary & $\begin{array}{c}\text { Pressure }^{\dagger} \\
(\mathrm{Pa})\end{array}$ & $\begin{array}{c}\text { Temperature } \\
(\mathrm{K})\end{array}$ & $\begin{array}{c}\text { Velocity } \\
(\mathrm{m} / \mathrm{s})\end{array}$ \\
\hline 1 & $\frac{\partial p}{\partial x}=0$ & $T=320$ & $\vec{v}=\left(U_{\infty}, 0\right)$ \\
2 & $p_{\infty}=0$ & $\frac{\partial T}{\partial y}=0$ & $\frac{\partial \vec{v}}{\partial y}=0$ \\
3 & $p_{\infty}=0$ & $\frac{\partial T}{\partial x}=0$ & $\frac{\partial \vec{v}}{\partial x}=0$ \\
4 & $\frac{\partial p}{\partial y}=0$ & $T=T_{s}$ & $\vec{v}=\left(0, v_{n}\right)$ \\
5 & $p_{\infty}=0$ & $\frac{\partial T}{\partial y}=0$ & $\frac{\partial \vec{v}}{\partial y}=0$ \\
\hline
\end{tabular}

(Note: $v_{n}$ is calculated from overall surface reaction rates; $v_{n}=0$ where reactions are turned off.)

${ }^{\dagger}$ Pressure is the reference hydrodynamic pressure.

The finite volume cells employed consist of an aspect ratio of 1 and size of $10 \mu \mathrm{m}$, resulting in 100, 000 cells for each simulation. Chemical residence time effects were inves- 
tigated by varying free-stream velocity up to $50 \mathrm{~m} / \mathrm{s}$ while keeping a fixed surface temperature of $1800 \mathrm{~K}$. This was done for both non-porous and porous surface reaction models. Temperature effects were studied by varying the surface temperature from 1800 to $2600 \mathrm{~K}$ for both non-porous and porous models with free-stream velocity of $40 \mathrm{~m} / \mathrm{s}$. Table 3.2 lists selected simulation results considered in the following discussions.

\begin{tabular}{lcc}
\hline Surface & $\begin{array}{c}\text { Velocity, } U_{\infty} \\
(\mathrm{m} / \mathrm{s})\end{array}$ & $\begin{array}{c}\text { Temperature, } T_{s} \\
(\mathrm{~K})\end{array}$ \\
\hline Non-porous & 40 & {$[1800,2000,2200,2400,2600]$} \\
Porous & 40 & {$[1800,2000,2200,2400,2600]$} \\
Non-porous & {$[30,40,50]$} & 1800 \\
Porous & {$[30,40,50]$} & 1800 \\
\hline
\end{tabular}

Table 3.2: Parametric values for free-stream velocity and surface temperature explored in the present study.

\subsection{Results and Discussions}

The converged steady-state solutions of reacting carbon surfaces for a range of free stream velocities and surface temperatures are presented here. For similar surface temperature and flow residence times, the effects of carbon surface porosity is explored by implementing the reaction models described in 2.5. As previously stated, the heterogeneous model for porous carbon provides consumption rates that orders of magnitudes greater than nonporous graphite. This important difference in model choice determines the contribution each reactant has on the reacting boundary layer profile, as well as the other parametric conditions discussed below. 


\subsubsection{Temperature Effects}

For a free-stream velocity of $40 \mathrm{~m} / \mathrm{s}$, Figs. $3.2 \mathrm{a}$ and $3.2 \mathrm{~b}$ show contour plots of $\mathrm{O}_{2}, \mathrm{CO}$, and $\mathrm{CO}_{2}$ mole fractions for the non-porous model at two extreme surface temperatures considered, $1800 \mathrm{~K}$ and $2600 \mathrm{~K}$, respectively. The higher surface temperature leads to increased carbon consumption at the surface resulting in significant $\mathrm{O}_{2}$ depletion near the surface. The $\mathrm{CO}$ formed at the surface is oxidized to form $\mathrm{CO}_{2}$ in the boundary layer, which is highest closest to the surface in the downstream, non-reacting surface region (for this high flow velocity). At locations nearest to the plate, the no-slip boundary condition causes the residence time to increase, which allows for the conversion of $\mathrm{CO}$ to $\mathrm{CO}_{2}$. The increase in temperature also increases the size of the reaction layer due to the increase $\mathrm{CO}$ flux from the surface and thermal expansion associated with heat release from $\mathrm{CO}$ oxidation in the gas-phase.

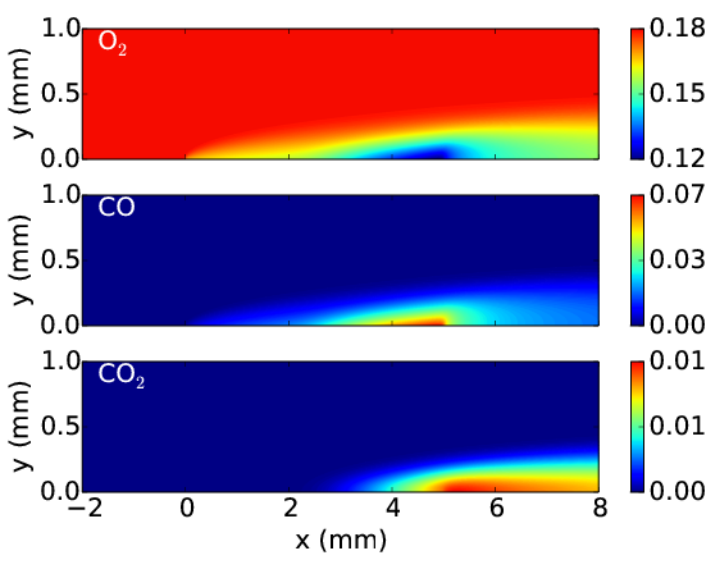

(a) $T_{s}=1800 \mathrm{~K}$
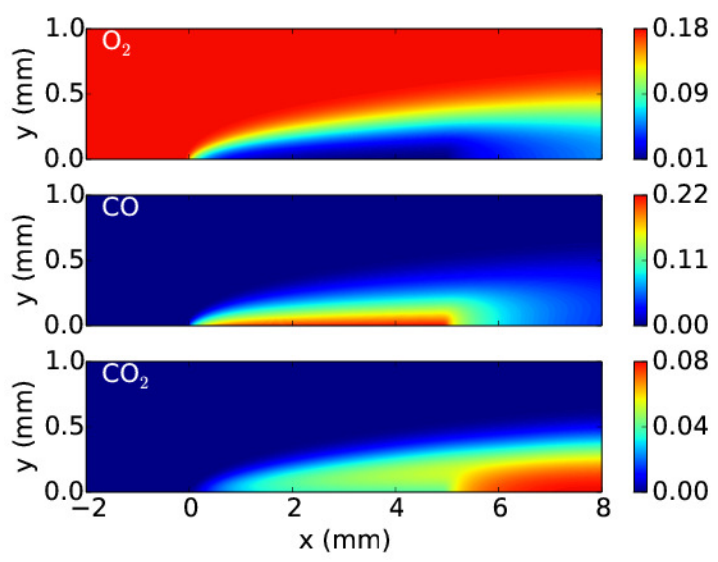

(b) $T_{s}=2600 \mathrm{~K}$

Figure 3.2: Two-dimensional contour plots of non-porous carbon surface reaction with freestream velocity of $40 \mathrm{~m} / \mathrm{s}$

For the porous carbon surface reaction model corresponding to about $25 \%$ porosity [25], a similar set of contour plots are shown in Figs. 3.3a and 3.3b for $\mathrm{O}_{2}, \mathrm{CO}$, and $\mathrm{CO}_{2}$ mole fractions at two surface temperatures of 1800 and $2600 \mathrm{~K}$. The higher reactivity of $\mathrm{O}_{2}$ of the porous model causes $\mathrm{O}_{2}$ mole fraction to be two orders of magnitude smaller than that of the nonporous case at $1800 \mathrm{~K}$. Similar to that of the nonporous model, for a velocity of 
$40 \mathrm{~m} / \mathrm{s}, \mathrm{CO}_{2}$ is highest in the region downstream of the non-reacting part of the flat plate where the blowing velocity is zero with increased flow residence time.

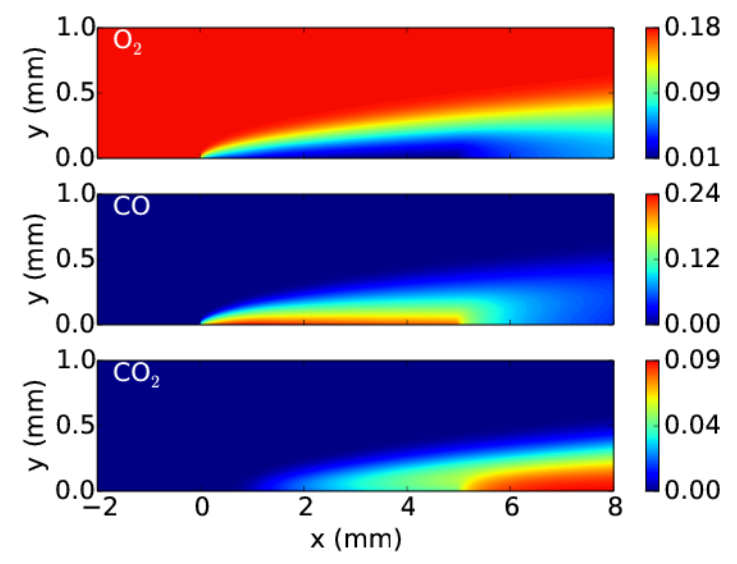

(a) $T_{s}=1800 \mathrm{~K}$
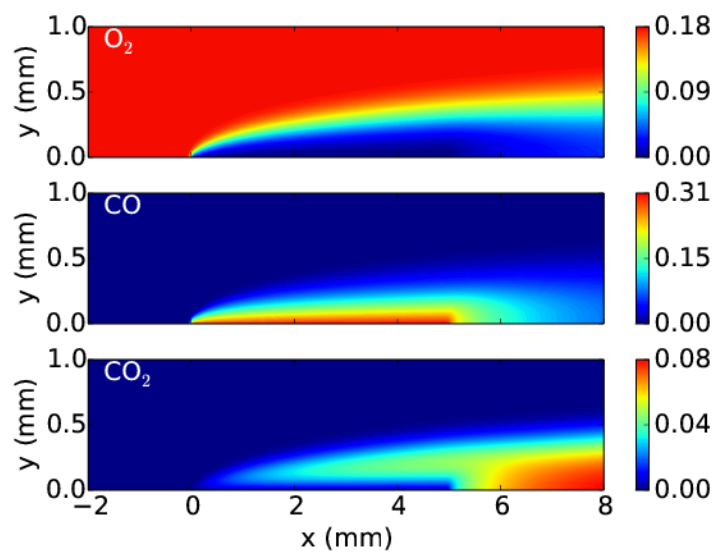

(b) $T_{s}=2600 \mathrm{~K}$

Figure 3.3: Two-dimensional contour plots of porous carbon surface reaction with freestream velocity of $40 \mathrm{~m} / \mathrm{s}$

The coupled effects of porosity and surface temperature controlling the two-dimensional contour plots shown above can be better understood by considering one-dimensional data normal to the surface. Figures 3.4a and 3.5a show a comparison of the $\mathrm{O}_{2}$ mole fraction along the surface for nonporous and porous surface reaction models, and for surface temperatures ranging from $1800 \mathrm{~K}$ to $2600 \mathrm{~K}$ with a fixed free-stream velocity of $40 \mathrm{~m} / \mathrm{s}$. Additionally, Figures $3.4 \mathrm{~b}$ and $3.5 \mathrm{~b}$ show the similar results for $\mathrm{CO}_{2}$ mole fraction variation. At the leading edge of the reacting sample, the porous carbon model shows a depletion of $\mathrm{O}_{2}$ compared to the nonporous model. Consequently, the mass loss rate towards the leading edge of the porous sample is much higher, leading to a non-uniform regression rate of the surface along the flow path. In both nonporous and porous cases the increase in temperature causes a sudden increase in $\mathrm{CO}_{2}$ with an eventual decrease in concentration at higher temperatures. This is due to the coupling of the surface and gas phase reactions, where at higher temperatures the surface reaction $\mathrm{C}_{s}+\mathrm{CO}_{2} \rightarrow 2 \mathrm{CO}$ with a larger activation energy of $64 \mathrm{kcal} / \mathrm{mole}$ becomes dominant. For both cases, $\mathrm{CO}_{2}$ decreased but the porous model has an order of magnitude less of $\mathrm{CO}_{2}$ along the surface due to the stronger reactivity. All 
of these profiles for species along the reacting surface change with temperature in both magnitude and shape making it necessary to include multidimensionality in carbon surface reactions where parallel flow is present.

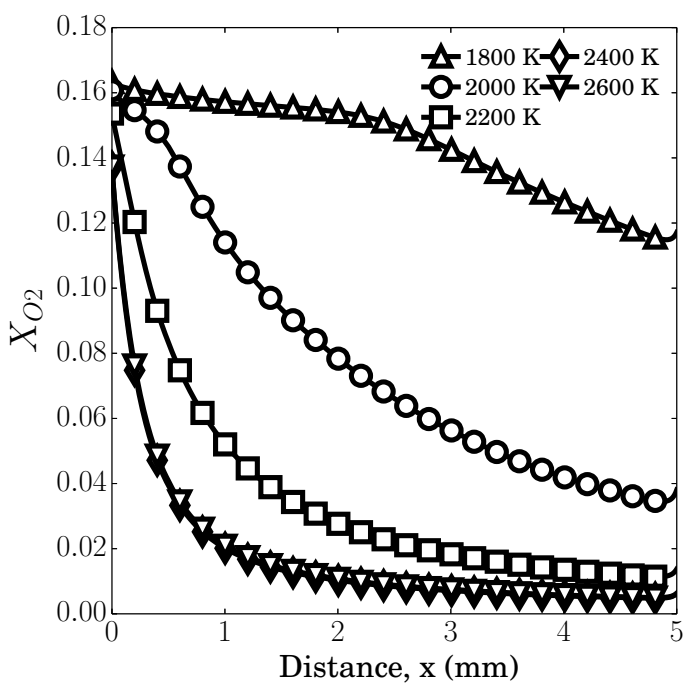

(a) $\mathrm{O}_{2}$ mole fraction

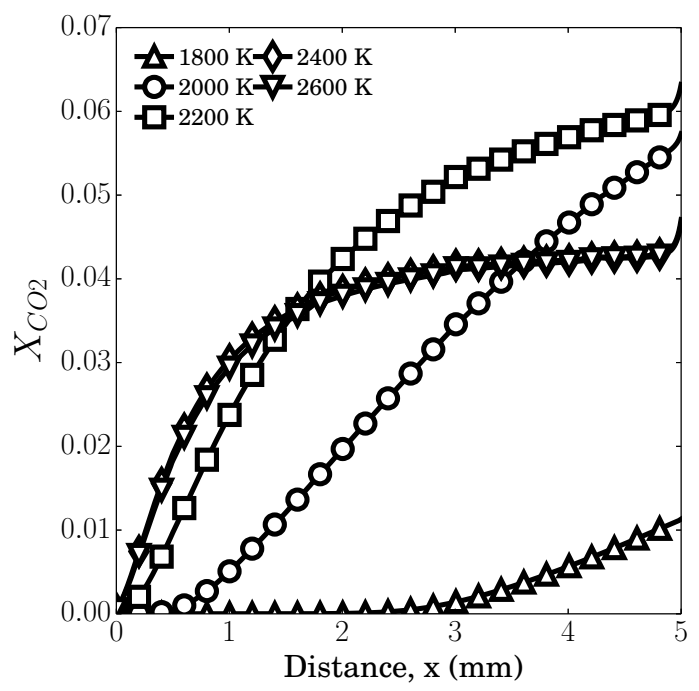

(b) $\mathrm{CO}_{2}$ mole fraction

Figure 3.4: Species concentrations along the reacting carbon plate for $\mathrm{CO}_{2}$ and $\mathrm{O}_{2}$ for temperatures ranging from 1800 to $2600 \mathrm{~K}$; line legends are the same for all figures

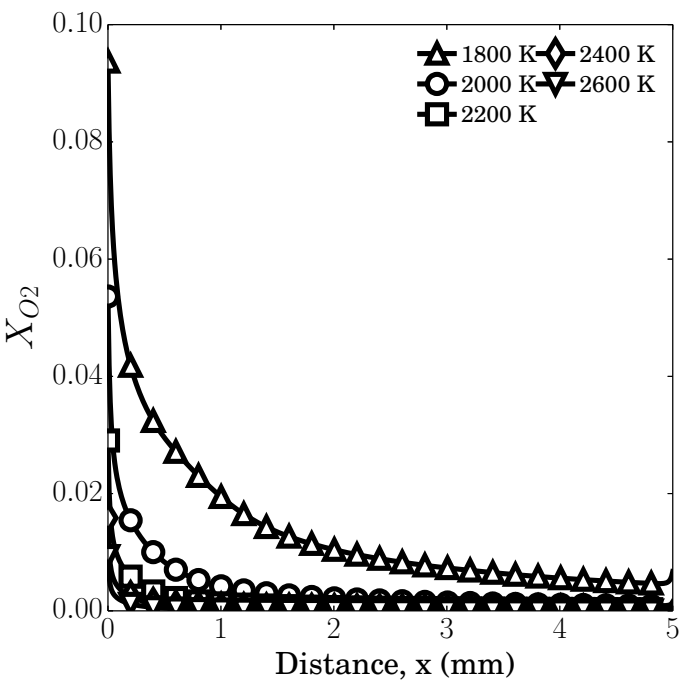

(a) $\mathrm{O}_{2}$ mole fraction

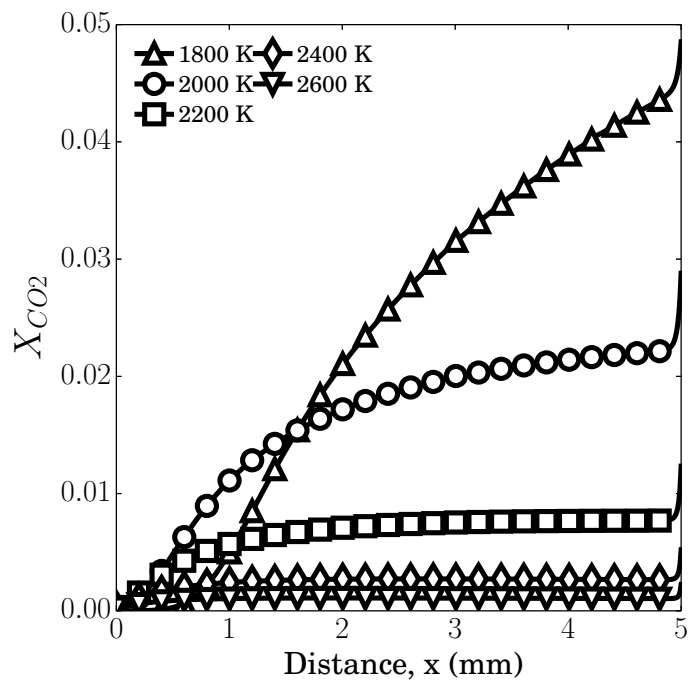

(b) $\mathrm{CO}_{2}$ mole fraction

Figure 3.5: Species concentrations along the reacting porous carbon plate for $\mathrm{CO}_{2}$ and $\mathrm{O}_{2}$ for temperatures ranging from 1800 to $2600 \mathrm{~K}$; line legends are the same for all figures 


\subsubsection{Residence Time Effects}

For a fixed surface temperature of $1800 \mathrm{~K}$ and for the nonporous model, Figs. 3.6a and 3.6b show contour plots of $\mathrm{O}_{2}, \mathrm{CO}$, and $\mathrm{CO}_{2}$ mole fractions with varying the free-stream velocity of 30 and $50 \mathrm{~m} / \mathrm{s}$, respectively. The influence of lower residence time associated with $50 \mathrm{~m} / \mathrm{s}$ flow on the gas-phase chemistry is demonstrated by the lack of conversion of $\mathrm{CO}$ to $\mathrm{CO}_{2}$. This suggests that a balance between diffusive and chemical source terms exists and that a numerical assessment can be utilized to understand local extinction based on overall $\mathrm{CO}$ to $\mathrm{CO}_{2}$ conversion rates. Additionally, an increase in velocity also reduces the $\mathrm{CO}_{2}$ mixing layer thickness and consequently increase the diffusive velocities normal to the reacting surface.

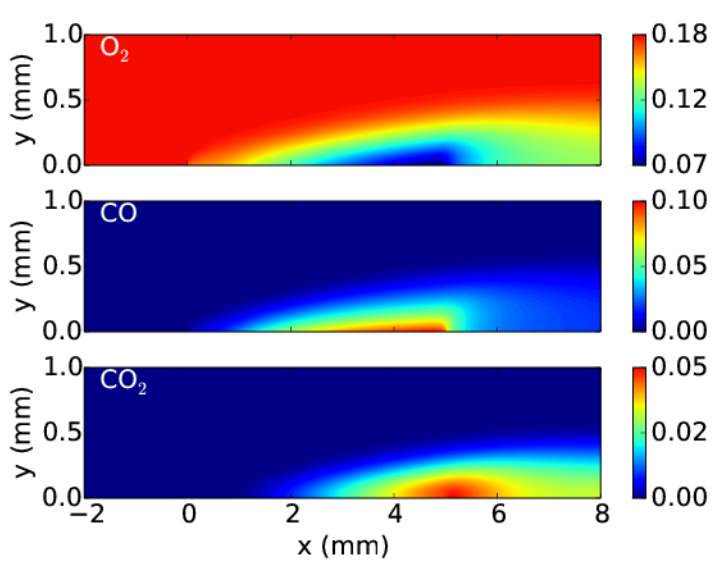

(a) Free stream velocity of $30 \mathrm{~m} / 2$

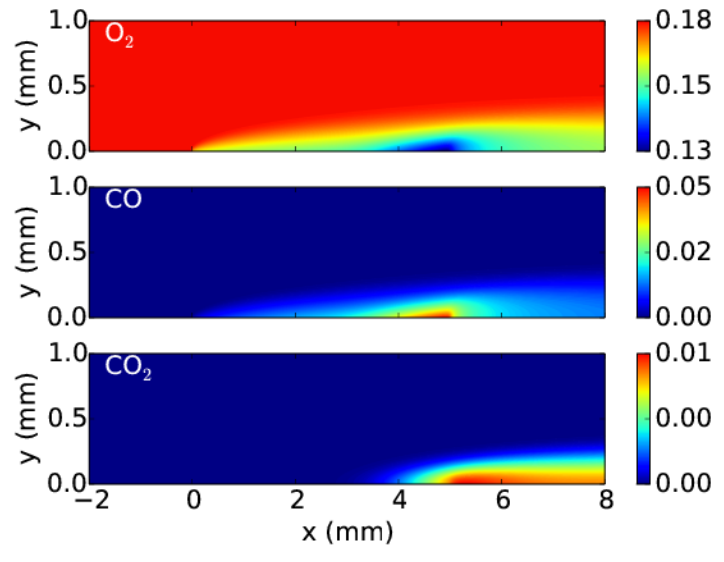

(b) Free stream velocity of $50 \mathrm{~m} / \mathrm{s}$

Figure 3.6: Contour plots of $\mathrm{O}_{2}, \mathrm{CO}$, and $\mathrm{CO}_{2}$ for nonporous surface reaction model, surface temperature of $1800 \mathrm{~K}$, and free-stream velocity of (a) $30 \mathrm{~m} / \mathrm{s}$ and (b) $50 \mathrm{~m} / \mathrm{s}$.

Once again, line plots of $\mathrm{CO}$ and $\mathrm{CO}_{2}$ along the reacting surface reveal the effects of porosity and varying free-stream velocities on both surface reaction rate producing $\mathrm{CO}$ and homogeneous oxidation of $\mathrm{CO}$, as shown in Figs. 3.7a and 3.7b. For the porous reaction model, velocity variation has a minimal effect on $\mathrm{CO}$ mole fraction indicating that the surface reactions are not controlled by transport at $1800 \mathrm{~K}$. In contrast, for the non-porous reaction model, a noticeable influence of flow velocity on $\mathrm{CO}$ mole fraction is seen and this can be attributed to the lower reactivity of the $\mathrm{O} 2$ reaction. Oxygen is depleted less in 
the nonporous reaction and other reactants, $\mathrm{OH}, \mathrm{O}$, and $\mathrm{CO} 2$, become lead contributors in the production of $\mathrm{CO}$. These other reactants are formed within the reacting layer which is shifted downstream with increase in free stream velocity. This $\mathrm{CO}$ concentration reliance on other reactants is quantified in detail in section 3.3.3.

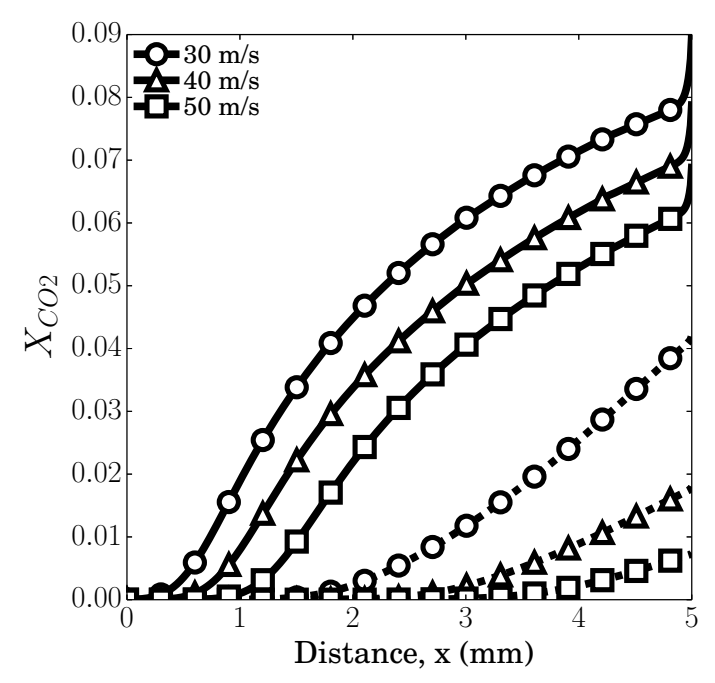

(a) $\mathrm{CO}_{2}$; nonporous - porous - -

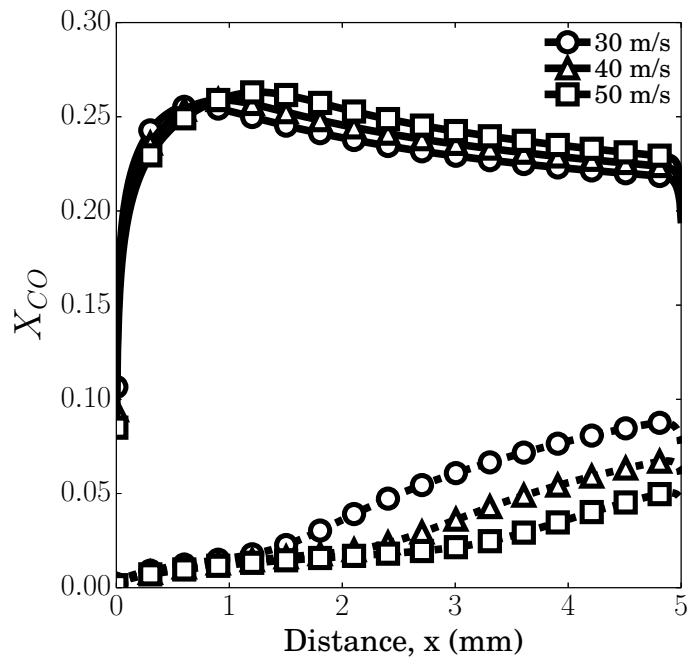

(b) $\mathrm{CO}$; nonporous - porous --

Figure 3.7: Species concentrations along the reacting carbon plate for $\mathrm{CO}_{2}$ and $\mathrm{CO}$ for free-stream velocities $30,40,50 \mathrm{~m} / \mathrm{s}$ with surface temperatuer $1800 \mathrm{~K}$

For the porous reaction model, velocity variation has a minimal effect on $\mathrm{CO}$ mole fraction indicating that the surface reactions are not controlled by transport at $1800 \mathrm{~K}$. In contrast, for the non-porous reaction model, a noticeable influence of flow velocity on $\mathrm{CO}$ mole fraction is seen and this can be attributed to the lower reactivity of the $\mathrm{O} 2$ reaction. Oxygen is depleted less in the nonporous reaction and other reactants, $\mathrm{OH}, \mathrm{O}$, and $\mathrm{CO} 2$, become lead contributors in the production of $\mathrm{CO}$. These other reactants are formed within the reacting layer which is shifted downstream with increase in free stream velocity. This CO concentration reliance on other reactants is quantified in depth in Section 3.3.3.

To further analyze the effects of flow residence time vs chemical time, the spatial locations where diffusive and chemical flux terms balance are analyzed here. From the steady state species conservation Eq. 2.4, one can readily extract the following terms, identified 
here as the ratio of chemical source term to diffusive flux term of species $i$,

$$
\nabla \cdot \rho \vec{v} Y_{i}-\nabla \cdot \rho D_{i} \nabla Y_{i}=w_{i}
$$

By dividing equation 3.1 by the $-\nabla \cdot \rho D_{i} \nabla Y_{i}$ term and rearranging an expression that quantifies the ratio of chemical source and species diffusion terms is found,

$$
\begin{aligned}
\frac{\nabla \cdot \rho \vec{v} Y_{i}}{-\nabla \cdot \rho D_{i} \nabla Y_{i}} & =\frac{w_{i}}{-\nabla \cdot \rho D_{i} \nabla Y_{i}}-1, \\
\frac{\nabla \cdot \rho \vec{v} Y_{i}}{-\nabla \cdot \rho D_{i} \nabla Y_{i}} & =R_{C / D, i}-1 .
\end{aligned}
$$

To avoid areas where $R_{C / D} \rightarrow \infty$ the ratio was redefined as

$$
R_{C / D, i}= \begin{cases}\frac{w_{i}}{-\nabla \cdot \rho D_{i} \nabla Y_{i}} & : Y_{i}>Y_{\min } \\ 0 & : Y<Y_{\min }\end{cases}
$$

Note: $R_{C / D, i}$ can be related to an appropriate Damköhler number.

Here, regions where the species of interest $i$ was less than $Y_{\min }=10^{-6}$ is not evaluated as the chemical reaction rate, $w_{i}$, is negligible. In the limit as $R_{C / D, i}$ approaches unity, the chemical source term balances with the diffusive flux term. At this point the convective term, from species conservation, must be negligible. The contour line shown in Fig. ?? identifies where $R_{C / D, C O_{2}}=1$. The regions where $R_{C / D, i} \leq 1$ can be identified as regions where gas-phase reactions cease to exist or where diffusion terms dominate. 


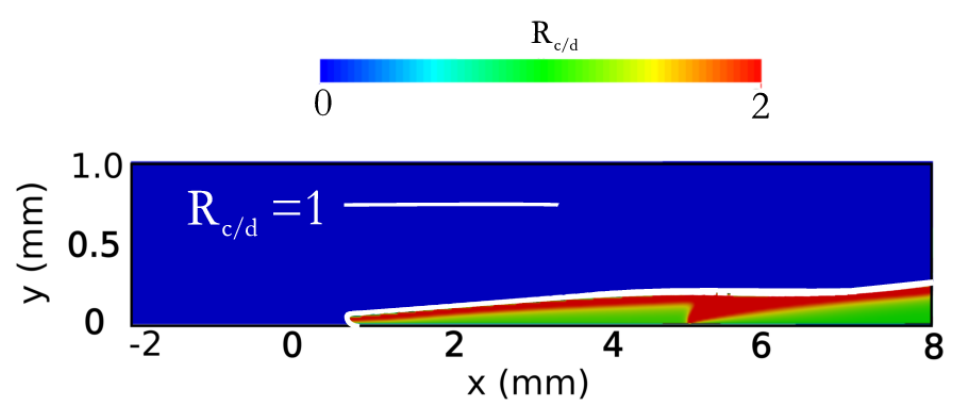

Figure 3.8: Contour plot of $R_{C / D}$ for $\mathrm{CO}_{2}$ using porous surface model, for a free-stream velocity of $50 \mathrm{~m} / \mathrm{s}$ with surface temperature of $1800 \mathrm{~K}$.

Note: For the purpose of illustrating $R_{C / D, i}=1$, the contour plot shown in Fig. ?? was limited to a maximum value of 2.0 because higher values were not important other than the fact that they are large.

Figures $3.9 \mathrm{a}$ and $3.9 \mathrm{~b}$ show a comparison of the $R_{C / D, C O_{2}}=1$ contour lines. As the free-stream velocity is varied from 30 to $50 \mathrm{~m} / \mathrm{s}$ the $R_{C / D, C O_{2}}=1$ contour lines shift in the downstream direction. The distance of this shift is dependent on the surface reaction model implemented. Specifically, for the nonporous reaction model, a shift in $R_{C / D, C O_{2}}=1$ lines is observed with increasing velocity, which is indicative of local extinction occurring. A shift is still apparent for the porous surface reaction model where higher CO fluxes from the surface occur and consequently yields higher $\mathrm{CO}_{2}$ production rates, however, the shift is less than that of the nonporous model. The present velocity range considered do not exhibit a local extinction phenomenon. However, further increase in free-stream velocity is expected to show a similar behavior as shown in Fig. 3.9a. 


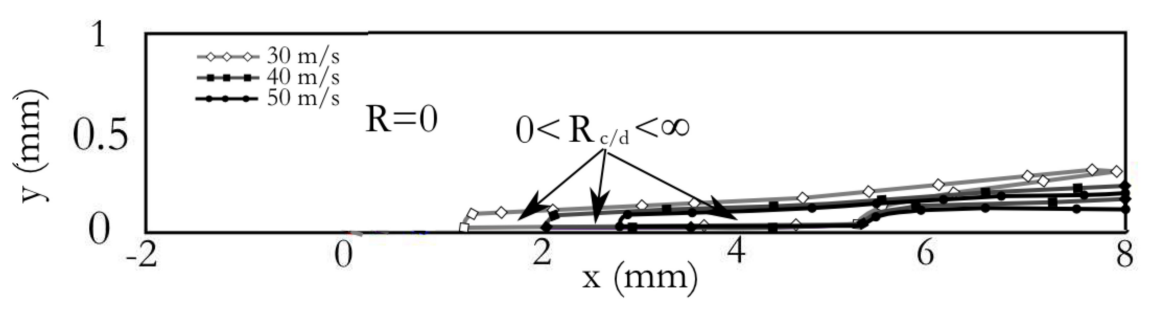

(a) Nonporous $\mathrm{Da}_{I I}=1$ contour lines for $\mathrm{CO}_{2}$

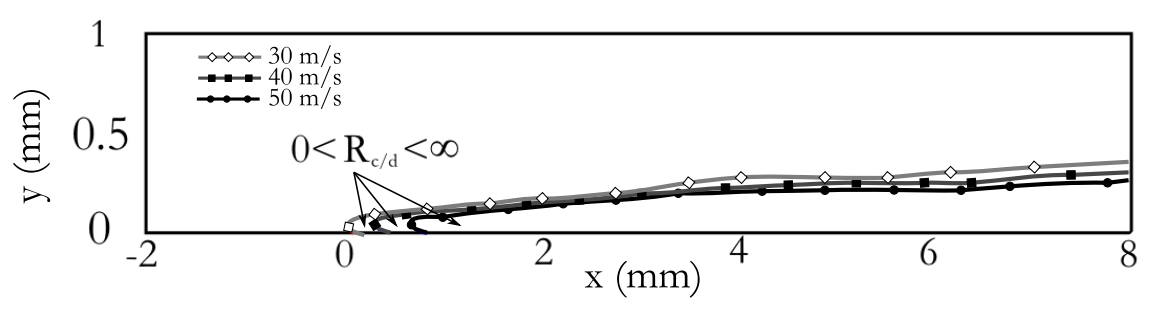

(b) Porous $\mathrm{Da}_{I I}=1$ contour lines for $\mathrm{CO}_{2}$

Figure 3.9: Two-dimensional contour lines for $\mathrm{CO}_{2} R_{C / D}$ number of unity for free-stream velocities 30,40 , and $50 \mathrm{~m} / \mathrm{s}$ with surface temperature $1800 \mathrm{~K}$.

Although the ratio $R_{C / D, i}$ gives detail to the overall response of a reacting surface to free-stream velocity, it does not give detailed information on convective, diffusive, and chemical source term variations. Figures $3.11 \mathrm{~b}$ and ?? show the values of the convective, diffusive, and mass production terms of species conservation equation of $\mathrm{CO}_{2}$ as a function of distance normal to the reacting surface, for the nonporous cases at 30 and $50 \mathrm{~m} / \mathrm{s}$, respectively. The data presented here were sampled at two stream wise locations along the reacting surface, namely at $x=4$ and $7 \mathrm{~mm}$. Here, Einstein notation was used to denote the convective, $\rho v_{j} \frac{\partial Y_{C O_{2}}}{\partial x_{j}}$, diffusive, $\frac{\partial \rho Y_{C O_{2}} V_{C O_{2}, j}}{\partial x_{j}}$, and mass production, $w_{C O_{2}}$, terms. 

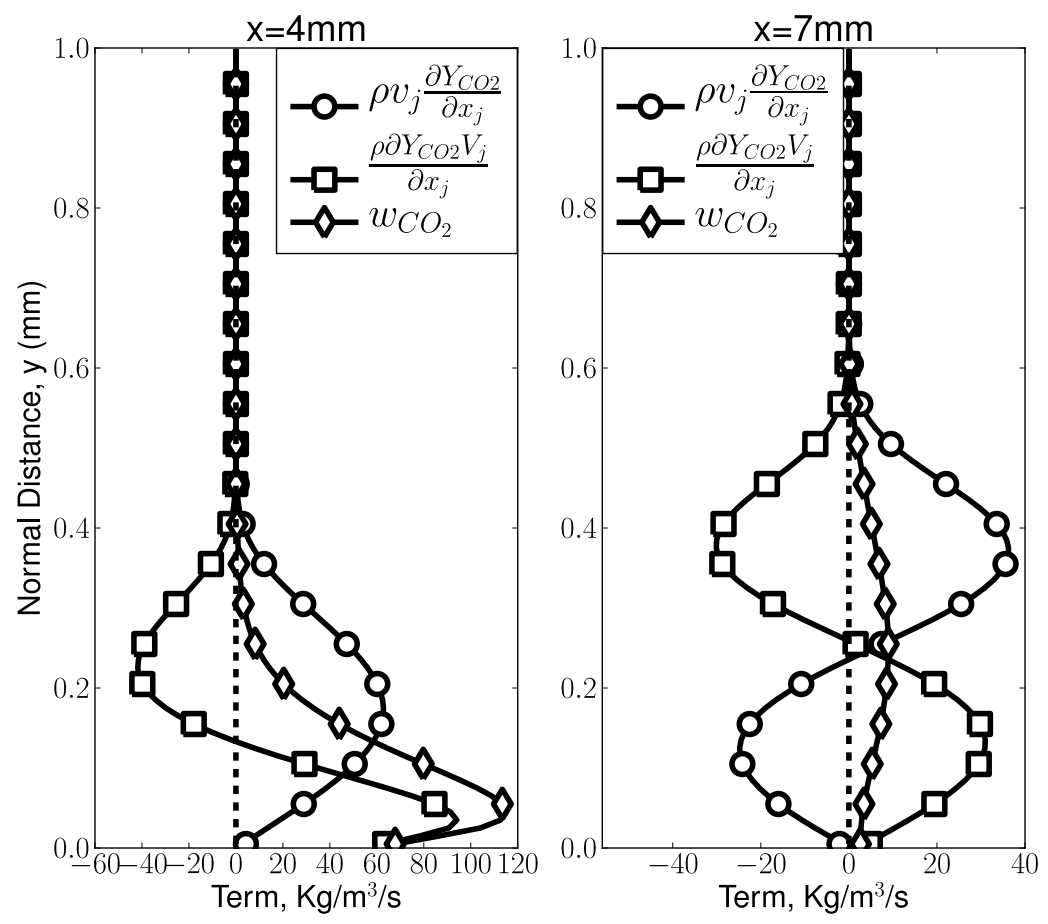

(a) Nonporous, $30 \mathrm{~m} / \mathrm{s}$
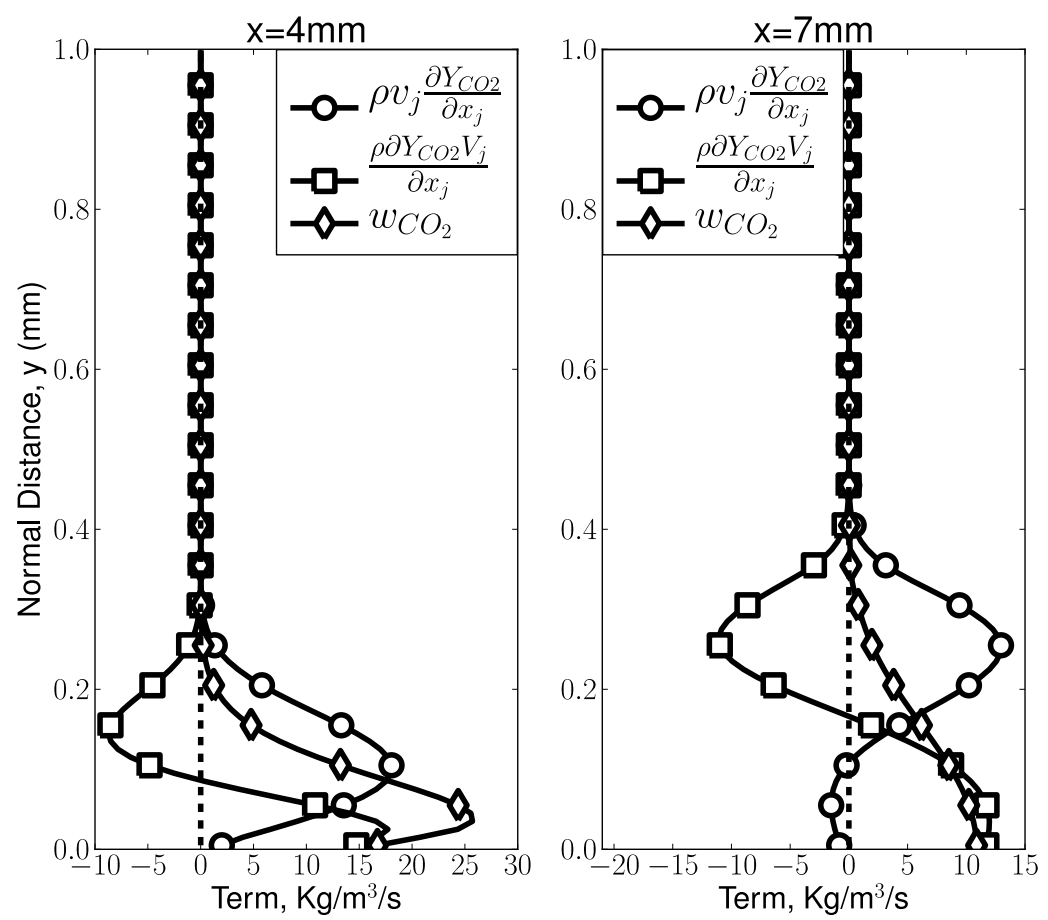

(b) Nonporous, $50 \mathrm{~m} / \mathrm{s}$

Figure 3.10: Terms of the $\mathrm{CO}_{2}$ conservation equation, $\frac{\rho v_{j} \partial Y_{C O 2}}{\partial x_{j}}+\frac{\partial \rho V_{j} Y_{i}}{\partial x_{j}}+w_{C O}=0$, for the nonporous model with 30 and $50 \mathrm{~m} / \mathrm{s}$ inflow velocity and surface temperature $T_{s}=1800$ $\mathrm{K}$ 

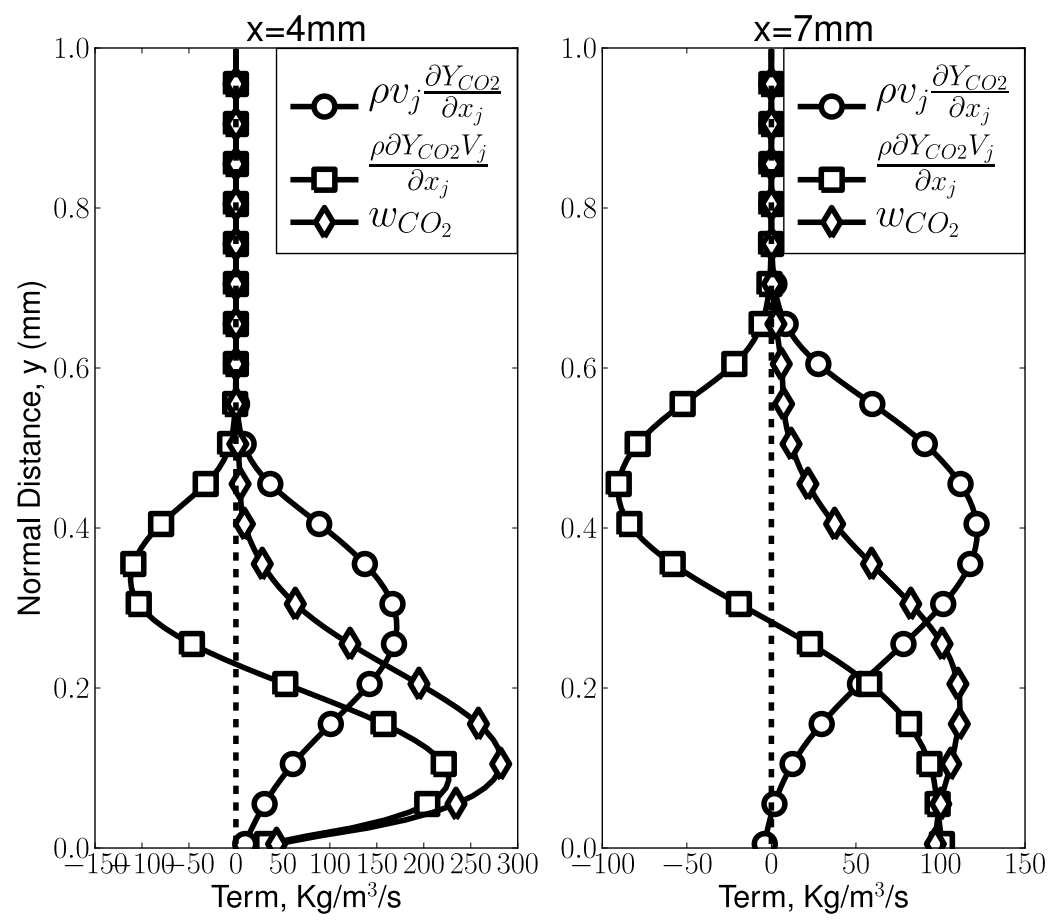

(a) Porous, $30 \mathrm{~m} / \mathrm{s}$
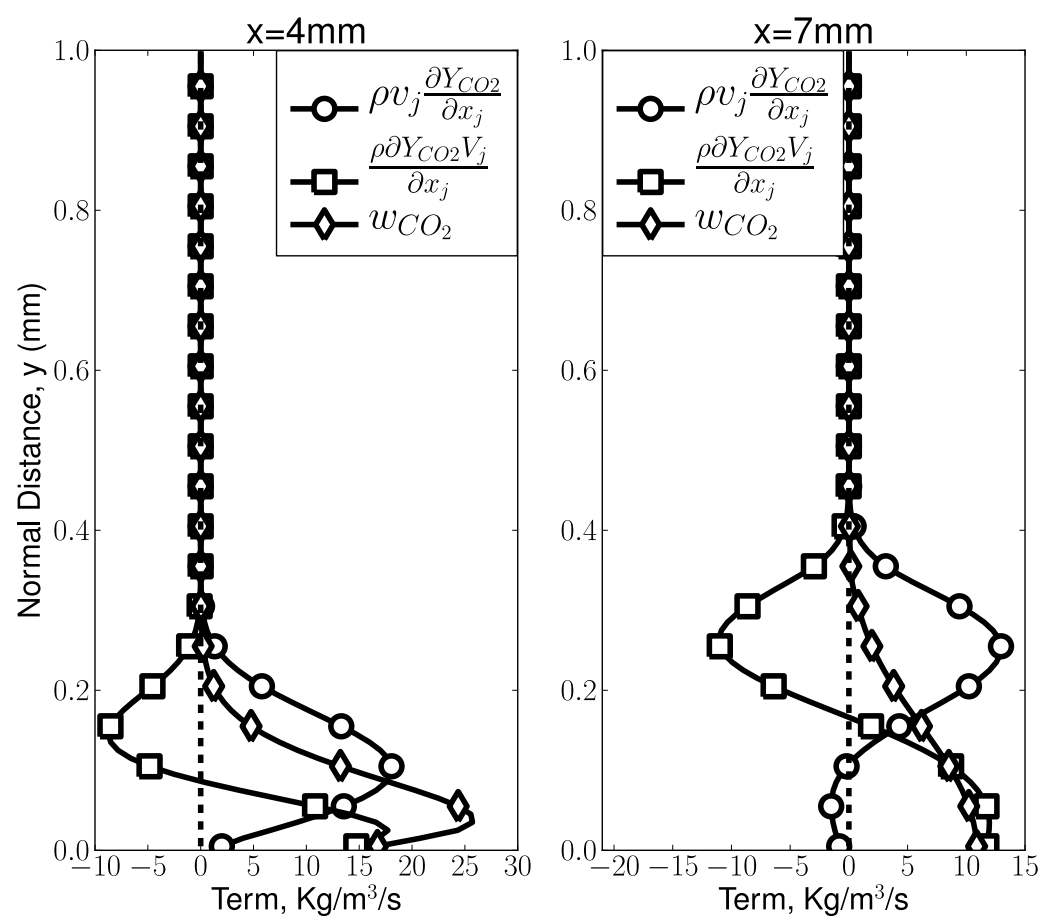

(b) Porous, $50 \mathrm{~m} / \mathrm{s}$

Figure 3.11: Terms of the $\mathrm{CO}_{2}$ conservation equation, $\frac{\rho v_{j} \partial Y_{C O 2}}{\partial x_{j}}+\frac{\partial \rho V_{j} Y_{i}}{\partial x_{j}}+w_{C O}=0$, for the porous model with 30 and $50 \mathrm{~m} / \mathrm{s}$ inflow velocity and surface temperature $T_{s}=1800 \mathrm{~K}$ 
Close to the plate the convective terms are small and the diffusive and mass production terms are balanced. As the distance from the plate increases, the reactivity diminishes and the convective and diffusive terms dominate. This is due to the decrease in temperature and increase in velocity within the momentum and thermal boundary layers. The nonreacting region has very small mass production rates, as $\mathrm{CO}$ is not produced at that location. The heights of these active layers decrease with increase in velocity; For example, the nonporous cases reduces from a height of $0.6 \mathrm{~mm}$ to $0.4 \mathrm{~mm}$, whereas the porous case reduce from 0.7 to $0.55 \mathrm{~mm}$ at $x=7 \mathrm{~mm}$. This is due to the thinning of the thermal boundary layer which decreases reactivity at distances normal to the plate. In addition, the decreased momentum boundary layer with increasing free-stream velocity causes the convective terms to dominate closer to the plate. This changes the overall balance in $\mathrm{CO}_{2}$ 's conservation equation and subsequently reduces $\mathrm{CO}_{2}$ 's peak values.

\subsubsection{Heterogeneous and Homogeneous Coupling}

There are 5 total heterogeneous, semi-global reactions that take place in both carbon surface models. In these models, CO, hydrogen molecules, and hydrogen atoms, are produced along the plate. These products are convected into the gaseous domain where they react to produce species that couple the heterogeneous and homogeneous reactions. This coupling combined with the fluid dynamics and surface temperature create various shapes and magnitudes of the reacting species within the reacting layer.

Each heterogeneous reaction contains a different reactant and contributes its own independent amount to the production of $\mathrm{CO}$. This overall production rate of $\mathrm{CO}$ is,

$$
w_{C O}^{\prime}=\sum_{k=1}^{5} \nu_{k} \frac{W_{C O}}{W_{k}} s_{k}^{\prime},
$$

where $W_{C O}$ is the molecular weight of $\mathrm{CO}, W_{k}$ is the molecular weight of the reactant species, $\nu_{k}$ is the stoichemetric coeffecient of $\mathrm{CO}$ in the heterogeneous reaction correspond- 
ing to reactant $k$, and $s_{k}^{\prime}$ is the surface mass rate of the reaction corresponding to reactant $k$. The coupling of heterogeneous and homogeneous reactions is extracted by the proportion each heterogeneous reactant contributes to the production of $\mathrm{CO}$. This proportion is evaluated as,

$$
\Phi_{k}=\frac{\nu_{k} \frac{W_{C O}}{W_{k}} s_{k}^{\prime}}{w_{C O}^{\prime}}
$$

where $\phi_{k}$ is the proportion of CO production as contributed by reactant $k$; all other symbols are the same as equation 3.3. This gives the contribution of a certain species to the production of $\mathrm{CO}$ in normalized units ranging from 0 to 1.

Figures $3.12 \mathrm{a}$ and $3.12 \mathrm{~b}$ depict the proportion $\mathrm{O}_{2}$ contributes to $\mathrm{CO}$ production along the surface for temperatures ranging from $1800 \mathrm{~K}$ to $2600 \mathrm{~K}$ for nonporous and porous models respectively.

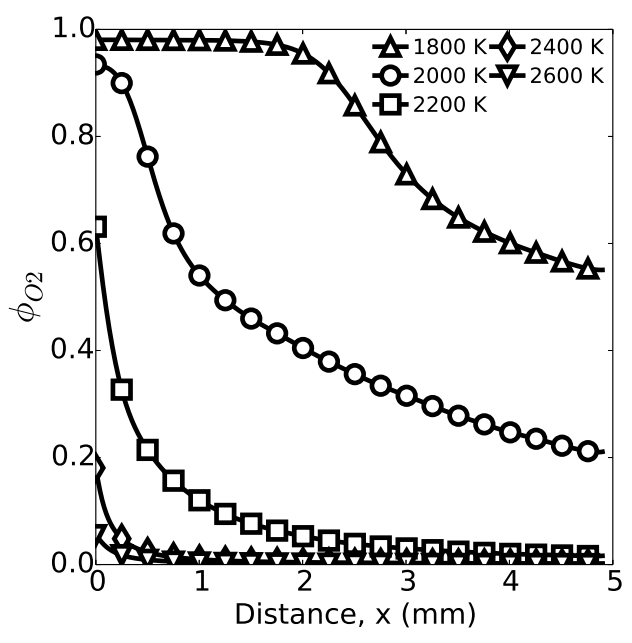

(a) Nonporous

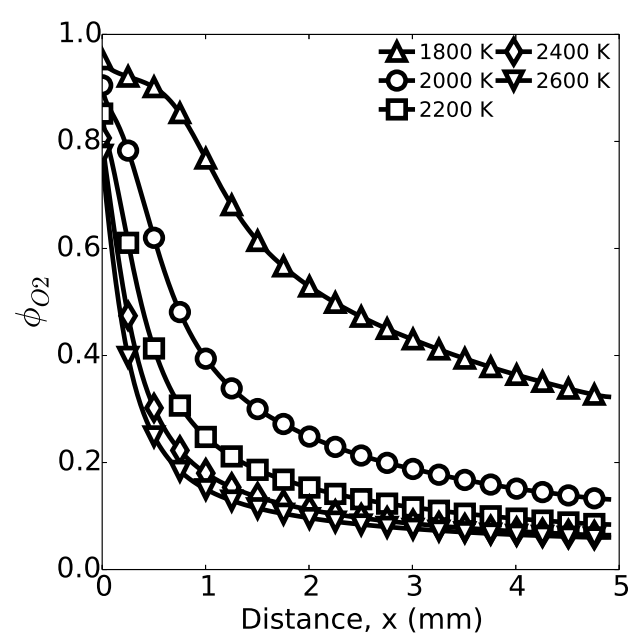

(b) Porous

Figure 3.12: Coupling of gaseous $\mathrm{O}_{2}$ and $\mathrm{CO}$ production, $\Phi_{O 2}$

$\mathrm{O}_{2}$ contributes more than 80 percent to $\mathrm{CO}$ production in the front end of the plate for both models at lower temperatures. As temperature increases, $\mathrm{O}_{2}$ becomes less of a role due to the increased reactivity in the gaseous domain, where $\mathrm{CO}_{2}, \mathrm{OH}$, and $\mathrm{O}$-atoms form within the thermal and reaction boundary layers. $\mathrm{O}_{2}$ is also consumed along the plate and is not reproduced by gaseous reactions which lowers its role in the axial direction. 
Comparitively, the role of $\mathrm{O}_{2}$ for high temperatures in the nonporous model is less than that of the porous model. This not not due to $\mathrm{O}_{2}$ 's depletion through consumption over the flat plate. Rather, it is due to the increase in roles of $\mathrm{OH}$ and $\mathrm{O}$-atom concentrations for $\mathrm{CO}$ production. Figures 3.13a and 3.13b show the production of $\mathrm{CO}$ due to $\mathrm{OH}$ and Figures 3.14a and 3.14b show the production of $\mathrm{CO}$ due to O-atoms.

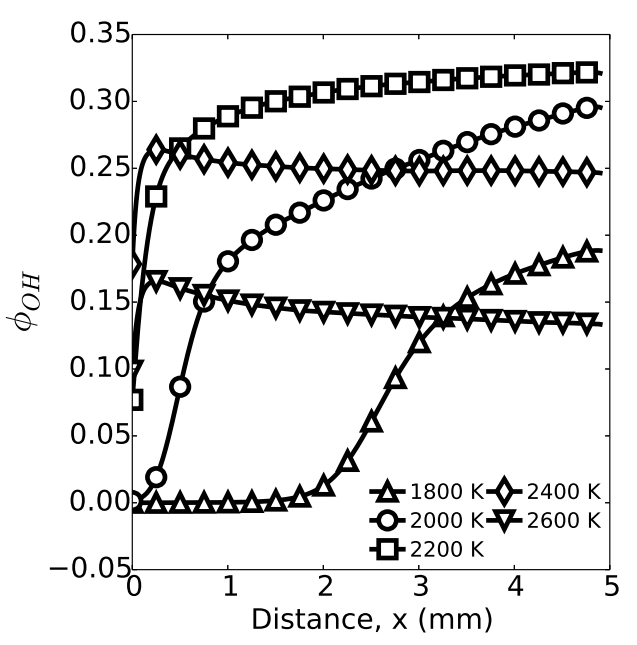

(a) Nonporous

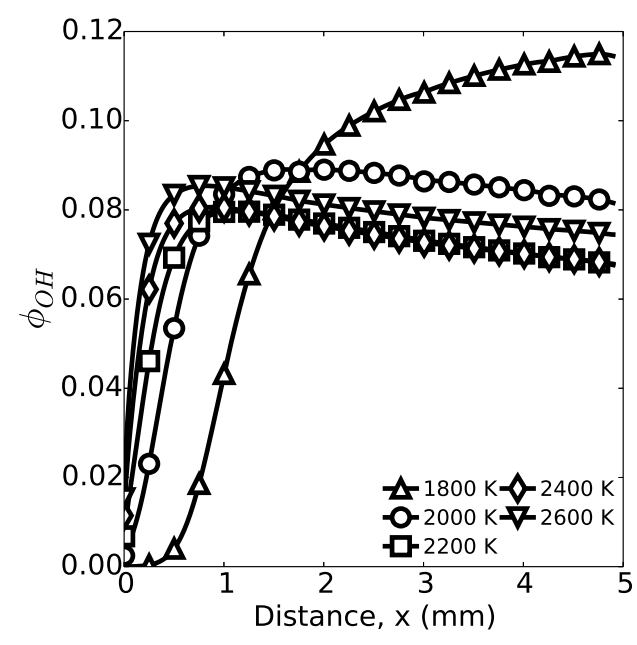

(b) Porous

Figure 3.13: Coupling of gaseous $\mathrm{OH}$ and $\mathrm{CO}$ production, $\Phi_{O H}$

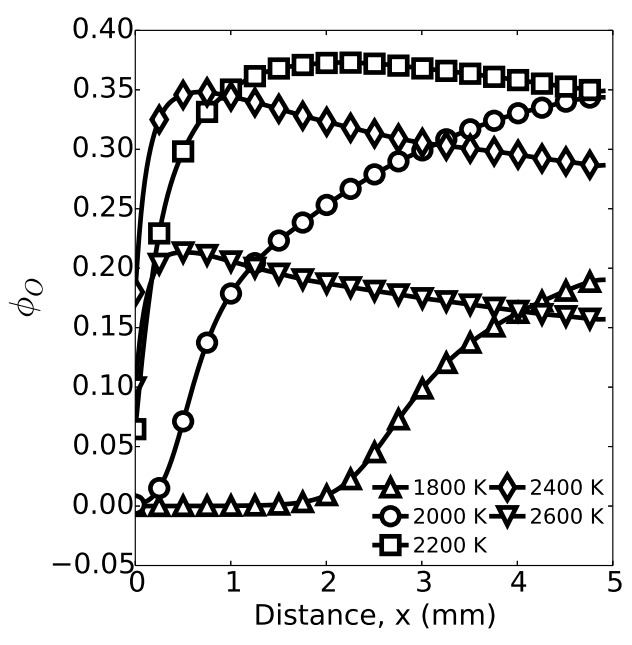

(a) Nonporous

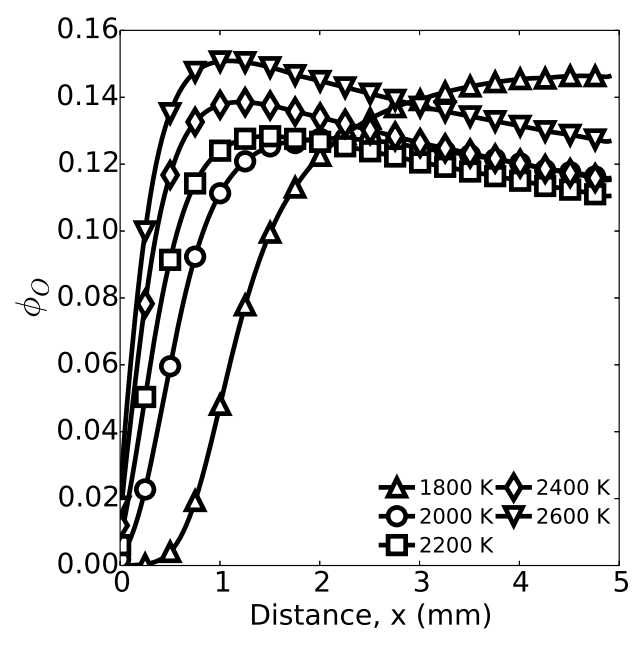

(b) Porous

Figure 3.14: Coupling of gaseous $\mathrm{O}$ and $\mathrm{CO}$ production, $\Phi_{O}$ 
Depending on temperature, $\mathrm{OH}$ and $\mathrm{O}$-atoms collectively produce 20 to 40 percent more of the $\mathrm{CO}$ than in the porous case. As previously mentioned, $\mathrm{O}_{2}$ 's reactivity for the porous model is two orders of magnitude greater than the nonporous model (see Figure 2.6). This causes $\mathrm{O}_{2}$ to be consumed less than in the porous case, which creates higher $\mathrm{O}_{2}$ concentrations in gaseous domain near the nonporous plate. The larger amount of $\mathrm{O}_{2}$ combined with high temperatures creates larger amounts of $\mathrm{OH}$ and $\mathrm{O}$, thereby increasing their role in $\mathrm{CO}$ production. Additionally, water reacts at the plate to form hydrogen molecules which accelerates the production of $\mathrm{OH}$ and $\mathrm{O}$ in the gaseous domain by the fast oxidation of hydrogen.

The reactivity of water also contributes to radical production by producting hydrogen molecules along the surface in addition to $\mathrm{CO}$. The hydrogen produced is oxidized in the gaseous domain which forms $\mathrm{OH}, \mathrm{O}$-atoms, and water. Figures 3.15a and 3.15b show the production of $\mathrm{CO}$ due to water for the nonporous and porous case respectively.

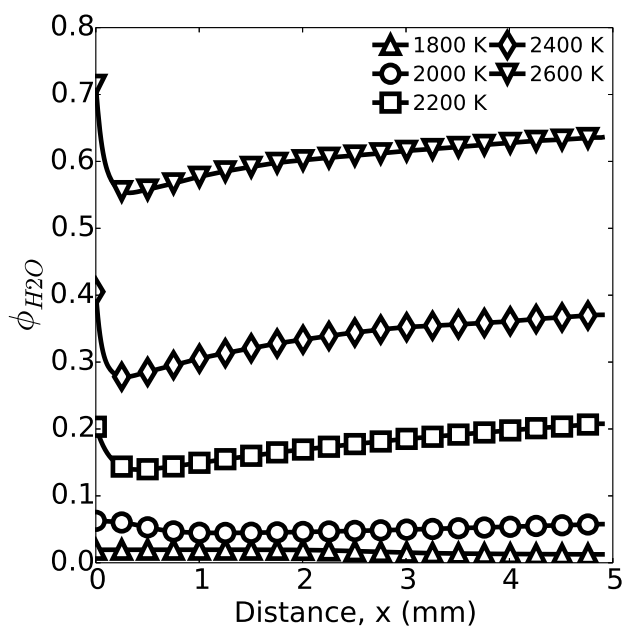

(a) Nonporous

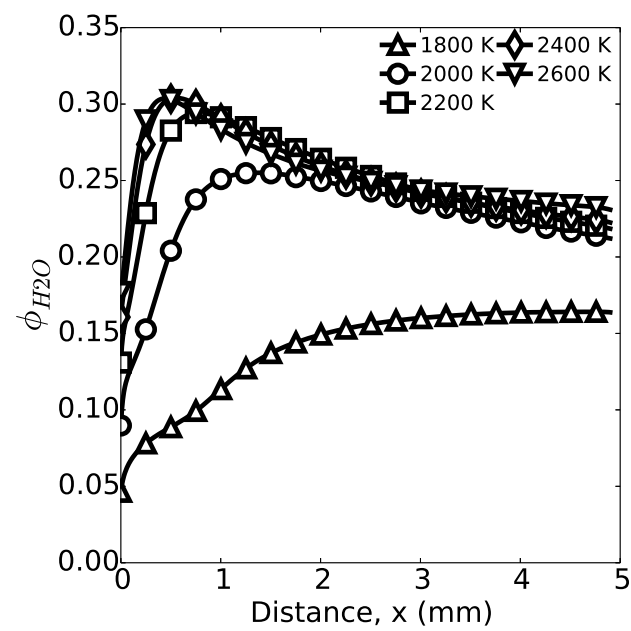

(b) Porous

Figure 3.15: Coupling of gaseous $\mathrm{O}$ and $\mathrm{CO}$ production, $\Phi_{\mathrm{H}_{2} \mathrm{O}}$

The contribution of water increases with inceased temperature for both cases. Water reaches above 60 percent of $\mathrm{CO}$ production in the nonporous case. A sudden increase in the nonporous case from 2400 to $2600 \mathrm{~K}$ is due to water exceeding the reactivity of $\mathrm{OH}$ and $\mathrm{O}$ - 
atoms. This is supported by the suddon reduction in $\mathrm{OH}$ and $\mathrm{O}$ contributions (see Figures $3.13 \mathrm{a}$ and $3.14 \mathrm{a}$ ) in the nonporous case. Water plays less of a role in the porous case due to $\mathrm{O}_{2}$ 's reactivity. When water reacts at the surface it creates both $\mathrm{CO}$ and hydrogen molecules. Gaseous hydrogen oxidationx assists in the formation of $\mathrm{OH}$ and $\mathrm{O}$ atoms along the surface.

Figures 3.16a and 3.16b show the production of $\mathrm{CO}$ due to $\mathrm{CO}_{2}$ for both nonporous and porous models, respectively.

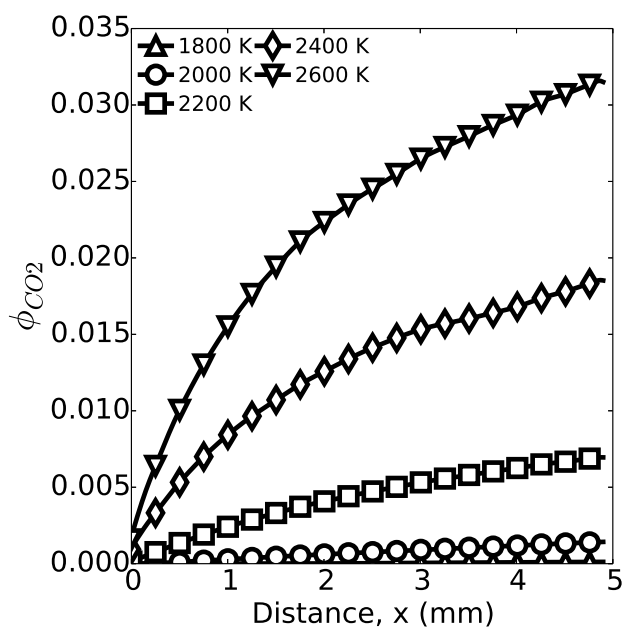

(a) Nonporous

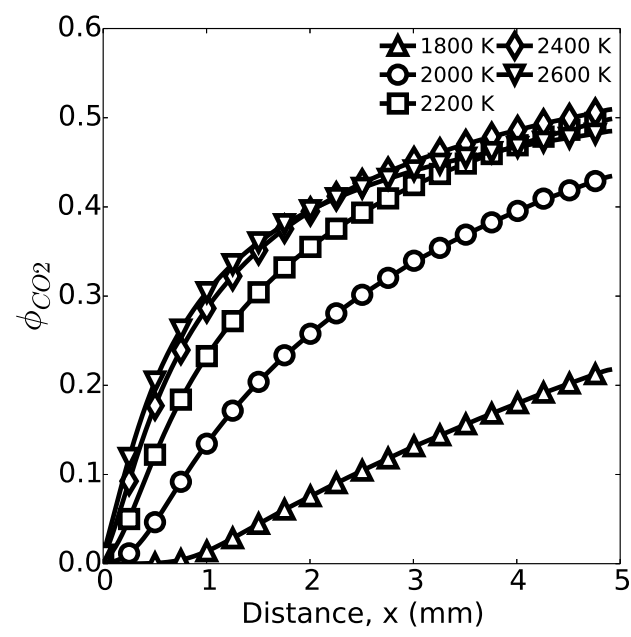

(b) Porous

Figure 3.16: Coupling of gaseous $\mathrm{CO}_{2}$ and $\mathrm{CO}$ production, $\Phi_{\mathrm{CO}_{2}}$

$\mathrm{CO}_{2}$ contributes two orders of magnitude less in the production of $\mathrm{CO}$ in the nonporous case than in the porous case. In the porous case, $\mathrm{CO}_{2}$ contributes 10 to 50 percent of $\mathrm{CO}$ production whereas the nonporous case contributes less than 1 percent. To test if the nonporous $\mathrm{CO}_{2}$ reaction was negligible the five temperature cases were simulated with the corresponding $\mathrm{CO}_{2}$ surface reaction removed. Figure 3.17 shows the mole concentration for $\mathrm{CO}$ along the plate for cases where the $\mathrm{CO}_{2}$ reaction was included (shown with solid line, -) and where the reaction was removed (shown with dashed line, --). 


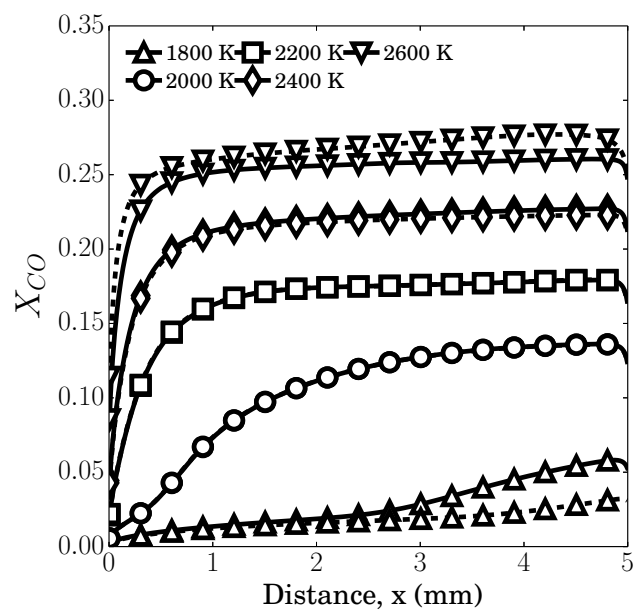

Figure 3.17: $\mathrm{CO}$ concentration along plate for $\mathrm{CO}_{2}$ reaction on (-) and $\mathrm{CO}_{2}$ reaction off (--)

The 1800 and $2600 \mathrm{~K}$ cases differ due to the exclusion of the $\mathrm{CO}_{2}$ reaction. The mid range temperatures, however, are close in both shape and magnitude. Figures $3.18 \mathrm{a} 3.18 \mathrm{~b}$ show concentrations for $\mathrm{CO}_{2}, \mathrm{OH}$, and $\mathrm{O}$-atoms in the normal direction to the reacting surface sampled at $\mathrm{x}=4 \mathrm{~mm}$ down the reacting plate for 1800 and $2600 \mathrm{~K}$ respectively. The reacting layers where the $\mathrm{CO}_{2}$ reaction was included are shown using a solid line, -, and the reacting layers where the reaciton was removed are shown with a dashed line, --.

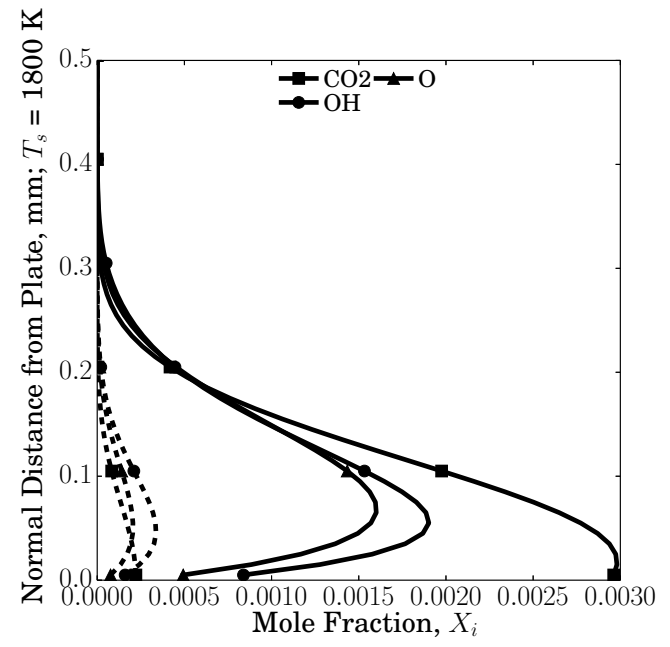

(a) Species concentrations for $\mathrm{CO} 2, \mathrm{OH}$, and $\mathrm{O}$-atoms normal from the plate sampled at $4 \mathrm{~mm}$ for $1800 \mathrm{~K}$

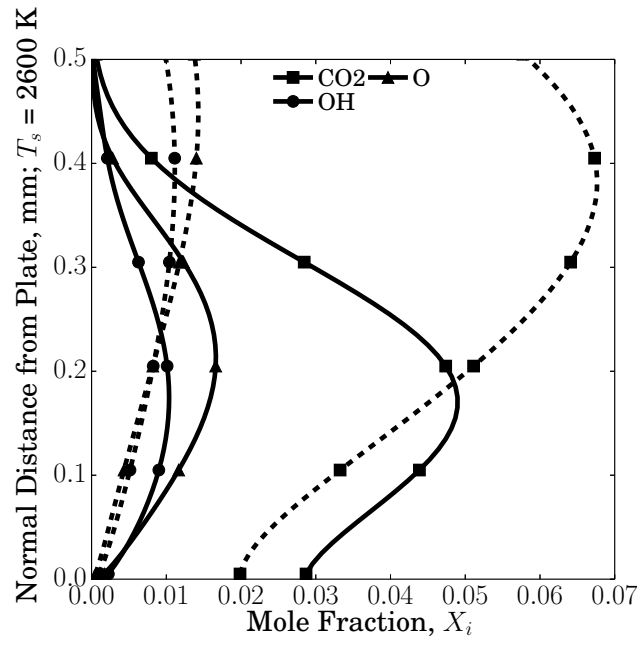

(b) Species concentrations for $\mathrm{CO} 2$, $\mathrm{OH}$, and $\mathrm{O}$-atoms normal from the plate sampled at $4 \mathrm{~mm}$ for $2600 \mathrm{~K}$

Figure 3.18: Reacting layer for cases with (-) and without (--) $\mathrm{CO}_{2}$ surface reaction. 
$\mathrm{CO}_{2}$ 's behavior at the reacting surface interface is determined by the inclusion of its mass consumption term. Without this term, the reaction structures differ in both magnitude and shape. This difference also determines the concentrations of $\mathrm{OH}$ and $\mathrm{O}$-atoms which are important reactants (see Figs. 3.13a and 3.14a) for CO production in the nonporous model. Despite $\mathrm{CO}_{2}$ small contribution to $\mathrm{CO}$ for nonporous cases, the surface reaction that consumes $\mathrm{CO}_{2}$ is still important. Therefore, it is necessary to include all reactions in these carbon surface heterogeneous models no matter how small of a role the reactant plays. 


\section{Chapter 4}

\section{Stagnant Flow Over a Reacting Carbon Surface}

\subsection{Background}

The planned stagnation flow experiments will use a striaght tube with fully developed parabolic flow to inject air normal to the carbon surface. The collimated laser beam that is used to heat the surface is routed concentrically through the same airflow tube. An illustration of this configuration is shown in Figure 4.1. The flow field that develops in this configuration is axisymmetric along the normal center-line of the flow field, which is easily described using cylindrical coordinates, $\mathrm{z}, \mathrm{r}$ and $\theta$. Any complexities added by the $\theta$-direction components, such as swirl, are unwanted in stagnation flow experiments and are neglected in the present work. This results in a two-dimensional domain that is dependent only on $\mathrm{r}$ and $\mathrm{z}$ directions. 


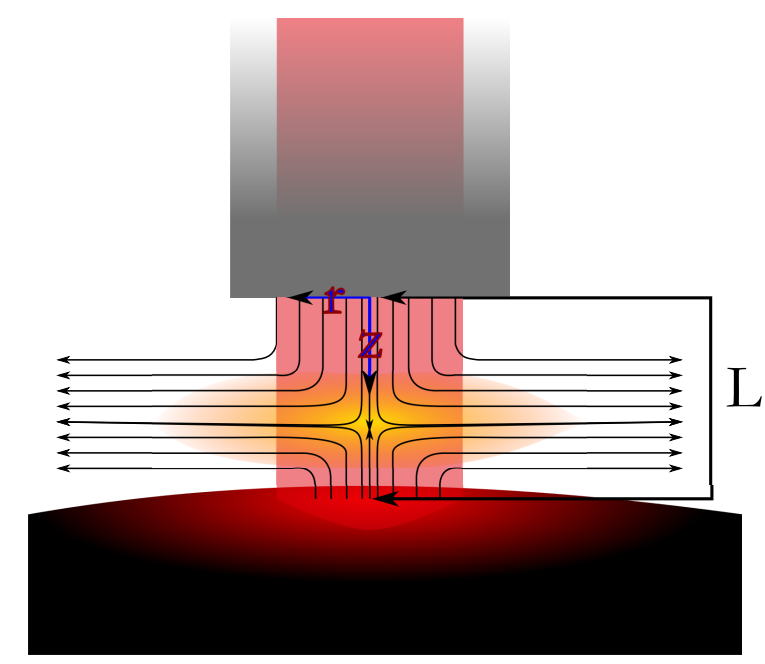

Figure 4.1: Reacting carbon surface stagnation flow experimental configuration, showing concentric laser (in red), and radial coordinates, $r$ and $z$.

Under certain conditions, the fluid dynamics of an axisymmetric impinging flow is reduced even further under a self-similarity approximation using a potential function, $U(z)$. This self-similarity function reduces the multidimensional reacting-Navier Stokes equations along the axis of symmetry to a set of ordinary differential equations that are computationally inexpensive to solve. Section 4.2 gives an overview of the quasi one-dimensional formulation and the limitations of its use.

The AFIT experiments will use a hyper-spectral imaging camera to explore the steadystate two-dimensional reacting layers in the stagnation flow configurations. The camera is limited to resolutions of $0.5-1.0 \mathrm{~mm}$ for species concentration measurements, a major weakeness of this instrument. Thus, with the objective of maximizing the reaction layer thickness along the axis of symmetry, the quasi one-dimensional formuation was used to guide the choice of separation distance, in-flow velocity, and laser effluence. The computational efficiency of the one-dimensional simulations allowed for large parameter sweeps and the results reported in Section 4.4.1 were used to guide the computational configurations.

Although the quasi one-dimensional formulation offers advantages in computation time and parameter manipulation, it lacks the ability of exploring the multidimensionality of the 
reacting surfaces. Two-dimensional axisymmetric simulations were used with the objective of complementing and better understanding the underlying physics of the two-dimensional reacting structure. The results of the two-dimensional simulaitons are discussed in Section 4.4.2. Supplemental information on reacting carbon surface flame detachment is presented in Section 4.4.1.

\subsection{The Quasi One-Dimensional Formulation}

The quasi one-dimensional formulation has been used for numerically estimating reacting stagnation flow problems for a variety of applications [36, 53, 61-63], including carbon surface reactions $[17,18,20,26,27]$. The formualtion is advantageous as it results in a system of ODEs that are solved to describe the physics along the reacting flow along the axis of symmetry. Three essential components are used in order to reduce these multidimensional stagnation flow configurations into the quasi one-dimensional formulation. The first of these components is the assumption that the radial velocity, $v_{r}$, is described using a self-similar function,

$$
v_{r}=r U(z)
$$

Here the self-similar function, $U(z)$, is a function purely of the axial position, $z$. The second component is the assumption that any radial gradients other than the radial gradient for pressure is negligible. This results in the continuity equation becoming purely a function of axial direction,

$$
\frac{\partial \rho v}{\partial z}+\frac{1}{r} \frac{\partial r \rho v_{r}}{\partial r}=\frac{\partial \rho v}{\partial z}+\frac{1}{r} \frac{\partial \rho U r^{2}}{\partial r}=\frac{\partial \rho v}{\partial z}+2 \rho U=0
$$


With these assumptions the radial component of the momentum equation becomes a function purely of axial direction as well

$$
\rho U^{2}+\rho v \frac{d U}{d z}-\frac{d}{d z}\left(\mu \frac{d U}{d z}\right)=-\frac{1}{r} \frac{\partial p}{\partial r}=\Lambda
$$

The third and final component is that $\frac{1}{r} \frac{\partial p}{\partial r}$ is constant (identified as the radial pressure curvature eigenvalue, $\Lambda$ ) along the axis of symmetry and is a consequence of the first and second components. Additionally the conservation of enthalpy and species are

$$
\rho v \frac{d h_{s}}{d z}-\frac{d}{d z}\left(\alpha \frac{d h_{s}}{d z}\right)+\frac{d}{d z}\left(\sum_{i=1}^{N} \rho h_{i}^{s} V_{x, i}\right)+\sum_{i=1}^{N} h_{i}^{c} w_{i}=0
$$

and

$$
\rho v \frac{d Y_{i}}{d z}-\frac{d}{d z}\left(\rho D_{i} \frac{d Y_{i}}{d z}\right)=w_{i}
$$

respectively. Equations 4.1-4.4 are ordinary differential equations that are easily discretized and solved. Computations for such a model usually take on the order of minutes to solve, which is an advantageous approach in the multidimensional modeling of reacting flows, which can take anywhere from days to weeks to converge with the use of multiple processors. This allows for computationally cost effective parameter sweeps to extract key trends that will exist experimentally, such as how in-flow velocity and surface temperature affect $\mathrm{CO}_{2}$ reaction layer thickness.

The three components of the quasi one-dimensional formulation must be satisfied in order to utilize its computational advantages. In summary the three components are:

1. The velocity field is self-similar, $v_{r}=r U(z)$.

2. Radial gradients, other than $\frac{1}{r} \frac{\partial p}{\partial r}$, are negligible.

3. The radial curvature of pressure is constant along the axis of symmetry, $\frac{1}{r} \frac{\partial p}{\partial r}=\Lambda$.

The first component is sensitive to the geometric conditions, such as convergent nozzles 
or straight tubes, that produces the incoming flow field. Consider Figure 4.2 where several axial velocity profiles in the radial direction are shown.

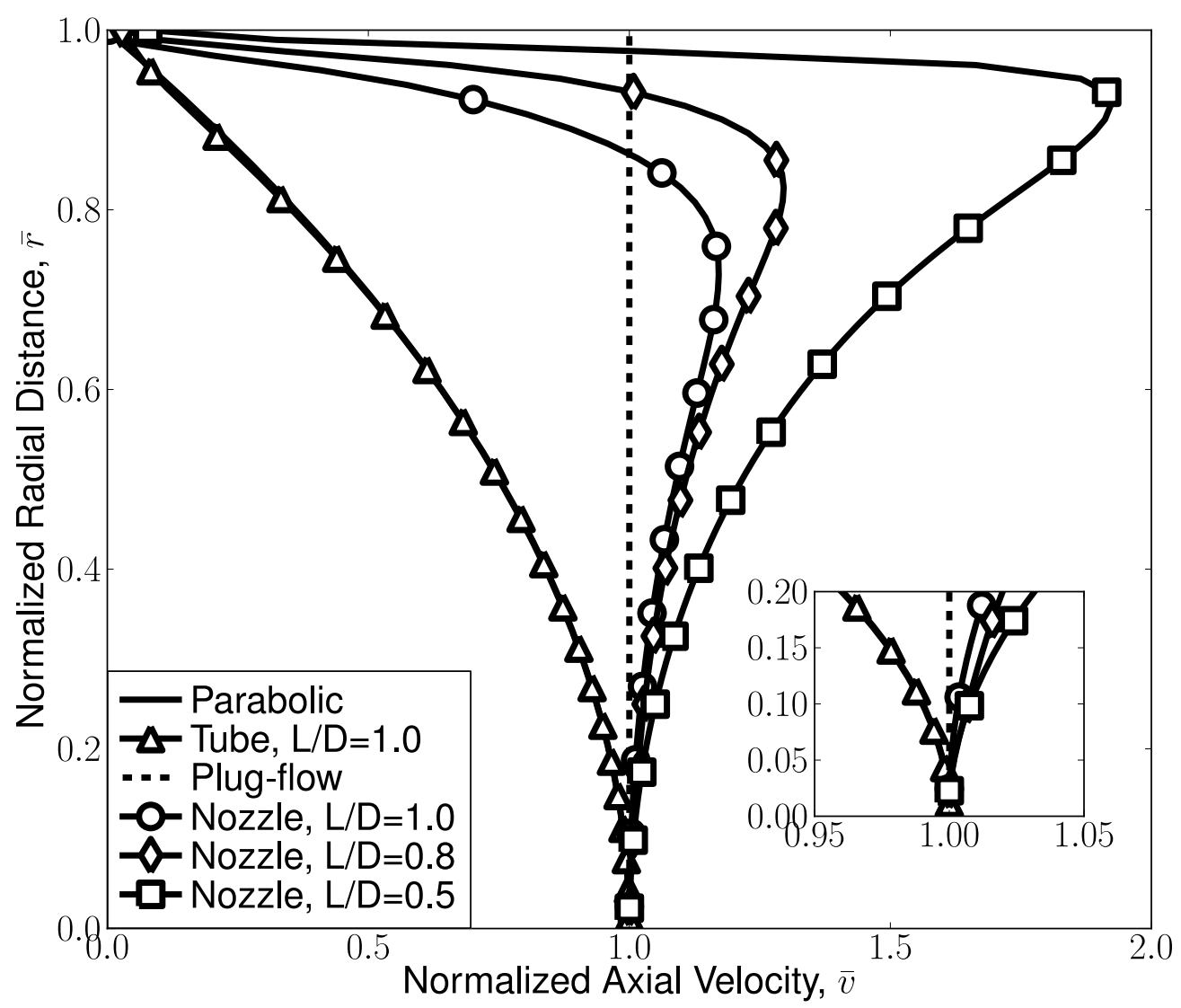

Figure 4.2: Axial velocity profiles with normalized velocity, $\bar{v}=v / v_{a x}$ ( $v_{a x}$ is axial velocity at the axis of symmetry) and normalized radial distances, $\bar{r}=r / R$ ( $R$ is the radius).

These velocity profiles are from several simulations of both straight-tube and convergent nozzles geometries with exit jets impinging normal to a flat surface. In the convergent nozzle case, by keeping exit diameter, D, constant and reducing the separation distance, $\mathrm{L}$, radial curvature of the axial velocity is increased. This radial curvature directly affects the axial direction velocity gradient, $\frac{\partial v}{\partial z}$, which is assumed to be zero in the quasi onedimensional formulation. Typically the zero velocity gradient is imposed by assuming that the self similar function is zero at the boundary $\left(\frac{\partial v}{\partial z}=0 \rightarrow U(z)=0\right.$ via Equation 
4.1) which cannot be satisfied by experiments using these geometries. For tube flow axial velocity profiles (also shown in Figure 4.2), radial curvature exists due to the parabolic nature of the fully-developed tube flow.

Rolon et al. [64] was the first to show that experimental stagnation flow configurations that do not use flow straighteners suffer from this non-uniformity in the axial velocity. Chelliah et al. [65] were the first to produce a method to correct for this non-uniformity by simply experimentally measuring $U(z)$ and imposing its value as a boundary condition within the quasi one-dimensional formulation. Furthermore, works by Dimotakis et al. $[66,67]$ and Sarnacki et al. [68] further highlighted the necessity of correcting $U(z)$ 's boundary conditions; Sarnacki et. al's work showed improvement in agreement between numerical modeling and experiments by practicing this method.

The second and third components of the quasi one-dimensional formulation have been addressed exclusively through numerical simulations. The reason is that $\Lambda$ and radial gradients are hard to measure experimentally. The first work to computationally explore these components is Frouzakis et al. (1998) [63]. In their axisymmetric simulations, $\Lambda$ was shown to deviate from a constant value even within the cold region of the reacting flow, which violates the third component. Their work also acknowledged possible radial gradients in their solutions, but only through a qualitative assessment. Frouzakis et al. went on to study counterflow flames that are produced by straight tubes, but did not return to further assess quasi one-dimensional formulation [69,70]. More recently, Mittal et al. [71] used axisymmetric simulations to show that including radial gradients (component two) affects solutions more than changes in $\Lambda$ (component three). Additionally, Bouvet et al. [72] came to the conclusion that the use of the quasi one-dimensional formulation fails at large separation distances and that subtle changes in velocity and $\Lambda$ within the thermal layer made the formulation inadequate. Despite these studies, no results have been produced supporting the use of the self-similarity function to describe flow fields from impinging parabolic flows. 
The most recent studies have investigated the cooling ability of tube jets on flat surfaces. Several groups, such as Duda et al. [73], Cornaro et al. [74], and Buchlin [75], experimentally investigated impinging tube jets through the use of visualization techniques (such as smoke streams or particle induced velocimetry). These studies focused on edge and wall vorticities formed by different geometries (flat surfaces with different concavities), by different separatation distances $(1.0<\mathrm{L} / \mathrm{D}<20.0)$, and by different Reynolds numbers. The intent was to further the understanding of impinging jet fluid dynamics in order to improve the cooling of surfaces. Specifically these groups found that the Nusselt number ${ }^{1}$ is a function radial distance along the surface. No mention of the quasi one-dimensional theory or any other theoretical approximation of the impinging fluid dynamics was mentioned in these studies.

A few groups have computationally explored impinging parabolic jets, with similar objectives of the experimental efforts. Sinha et al. [76] used numerical investigations to examine the effect that the separation distance has on the cooling of heated surfaces, finding that heat transfer is a function of L/D. Ramezanpour et al. [77] investigated the heat transfer of a an impinging jet normal to a heated plate at different inclinations, finding that the change in inclination changed the Nusselt number along the surface, as well as the stagnation point. Additional notable works are the Nakazawa et al. [78] study that investigated flame synthesis of carbon nanotubes with impinging jets and the Huang et al. study [79], which investigated the use of nano particles to assist heat transfer. Out of all these studies, no mention of the quasi one-dimensional theory was made.

The exclusion of the quasi one-dimensional formulation in these studies does not mean analytical solutions are missing for the impinging tube flows. Sholtz et al. [80] developed a theoretical solution for impinging parabolic tube flows for various separation distances. The solution agreed well with experiments with large separation distances. The study also showed that the Reynolds number has an effect on radial pressure distributions along the

\footnotetext{
${ }^{1}$ Nusselt number is a ratio of convective heat transport (removed by fluid) to conductive heat transport
} 
surface when $\mathrm{L} / \mathrm{D}<1.0$, but no effect was seen at larger separation distances, $\mathrm{L} / \mathrm{D}>1.0$. This indicates a possible free-floating regime for tube jet scenarios. More recently, Phares et al. [81] used eigenfunction expansion to solve the stream-function PDE for rotational, impinging jets with arbitrary non-uniform velocity profiles. A numerical method was used to iterate for the vorticity present near the surface. The method was not successful for close separation distance, but showed strong agreement for separation distances of $L / D>8.0$.

To date, Frouzakis et al. [63] performed the only reacting flow study that considered parabolic axial velocity profiles in two dimensional stagnation point flows. The study showed that results from two dimensional simulations with parabolic velocity boundary conditions deviated more from the quasi one-dimensional formulations than the two dimensional plug flow simulations, which confirmed that the plug flow velocity profile is ideal. The boundary condition was also prescribed as parabolic flow at the exit of the nozzle, which did not allow the separation distance to affect the tube's outflow velocity profiles. No recommendations were given on how to mitigate the variations found in this study.

The lack of information on the quasi one-dimensional formulation's applicability to tube jet flows raises the following research questions:

1. Does the quasi one-dimensional formulation apply to parabolic tube flows? and, if so,

2. What is the ideal experimental configuration to use the quasi one-dimensional formulation?

These research questions together create a whole new research objective that was realized in this work. 


\subsection{Solution Domain and Procedure}

Figure 4.3 shows an illustration of the two-dimensional computational domains used for the stagnation flow configuration with tube nozzle radius, $\mathrm{R}$, and separation distance is length, L. Streamlines are included to illustrate the expected direction of flow. Figure 4.3a is the tube flow configuration with radius $\mathrm{R}$ and Figure $4.3 \mathrm{~b}$ is a simplified version of the tube flow configuration which does not include the flow within the tube. In Figure 4.3a the simulated surface length is scaled by factor a, where $a>2$. Likewise, the outflow domain height is scaled by factor $\mathrm{b}$, with $b>1.0$. The scaling factor $\mathrm{b}$ was changed in order to remove the possibility that flow entrainment would alter the solutions and is discussed in later sections.

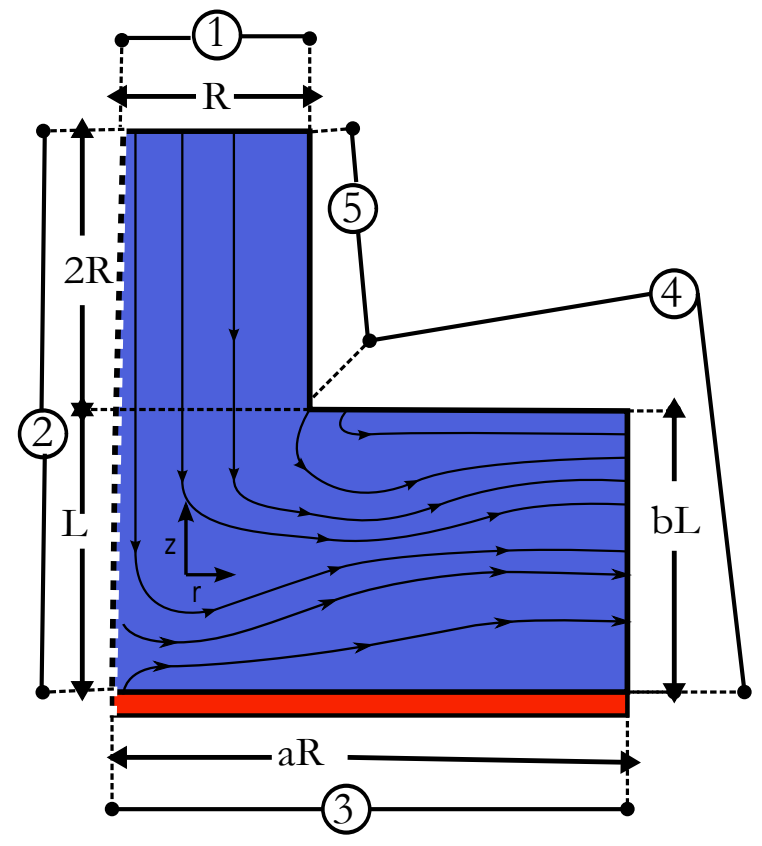

(a) Tube Flow

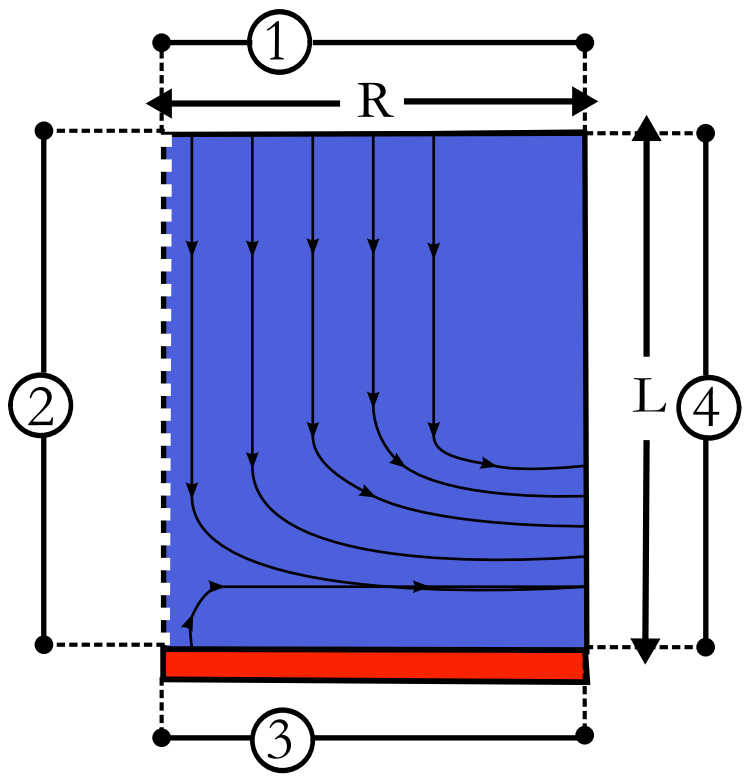

(b) Nozzle Flow

Figure 4.3: Two-dimensional axisymmetric domains.

Instead of the straight, non-slip boundary 5 (the wall), a wall function was used in addition to these two main configurations to simulate a convergent nozzle flow. This configuration was used to generate the results shown in Figure 4.2 and additional differences between nozzle and tube flows will be reported in the later sections. 
Table 4.1: List of boundary conditions for Figure 4.7's computational domains.

\begin{tabular}{|l|c|c|c|c|}
\hline Boundary & $T$ & $p$ & $\vec{v}$ & $Y_{i}$ \\
\hline 1 & $300 \mathrm{~K}$ & $\frac{\partial p}{\partial z}=0$ & $\vec{v}=(0, v(r), 0)$ & $\begin{array}{c}Y_{O 2}=0.21, \\
Y_{H 2 O}=0.02, \\
Y_{N 2}=0.77, \text { and } Y_{i}=0 \\
\text { for } i \neq \mathrm{O}_{2}, \mathrm{H}_{2} \mathrm{O}, \mathrm{N}_{2}\end{array}$ \\
\hline 2 & Axis of & Axis of & Axis of & Axis of Symmetry \\
\hline 3 & Symmetry & Symmetry & Symmetry & $\begin{array}{c}Y_{i} \text { calculated from } \\
\text { surface reactions }\end{array}$ \\
\hline 4 & $T_{s}$ is fixed & $\frac{\partial p}{\partial z}=0$ & $\vec{v}=\left(0, v_{n}(r), 0\right)$ & $\frac{\partial Y_{i}}{\partial r}=0$ \\
\hline $5^{\dagger}$ & $\frac{\partial T}{\partial r}=0$ & $p=0$ & $\frac{\partial \vec{v}}{\partial r}=0$ & $\frac{\partial Y_{i}}{\partial r}=0$ \\
\hline
\end{tabular}

${ }^{\dagger}$ Boundary 5 is not included in the simplified mesh as it does not simulate the walls

Table 4.1 lists the boundary conditions used for the domains illustrated in Figure 4.3. Several parts of this table need further clarification. The surface temperature, $T_{s}$ can be specified as either as a constant, where it will usually be $>1600 \mathrm{~K}$ to promote reactivity, or a radial varying function, $T_{s}=T_{s}(r)$, to match the energy laser profile. Additionally, a thermal balance at an interface may be used to determine $T_{s}$; the effects of thermal balance are reported in Chapter 5. This chapter focuses on an prescribed values of $T_{s}$.

The axial velocity, $v_{z}$, was also varied in addition to surface temperatures. For uniform, plug-flow, velocity fields, $v_{s}$ was imposed as a constant value, $V_{p l u g}$. This constant value varied depending on case, but was usually confined to $V_{p l u g}<10 \mathrm{~m} / \mathrm{s}$, as higher velocities caused small reaction layer thicknesses. This constant velocity boundary condition was 
only used in the simplified case, as constant velocities are not a physical outcome in tube flows. Instead, the tube flow case used a parabolic velocity profile. By matching the mass flow rate of the tube to the mean, or plug, flow profile, the velocity profile of axial velocity is written as

$$
v_{z}(r)=2\left(\frac{V_{p l u g}}{R^{2}} r^{2}-V_{p l u g}\right) .
$$

Equation 4.5 is for ideal tube-flows that are not affected by downstream gradients; in this case, the presence of the stagnation surface would cause the parabolic exit profile to deviate from the fundamental solution that results in Equation 4.5. To account for changes due to the stagnation surface, the parabolic velocity profile was imposed upstream of the tube exit. The distance of this boundary condition was tested and tube lengths greater than $1.5 R$ were found to converge to the same exit velocity profile. The tube length was kept constant at $2 R$ for all results reported here.

The last boundary condition needing clarification is the velocity and species concentrations along the reacting surface, boundary 3 . The normal velocity, in this case $v_{z}=v_{n}$, is updated using the reaction rates from the heterogeneous reaction models,

$$
v_{n}=\frac{\sum_{i=1}^{N} w_{i}^{\prime}}{\rho} .
$$

Equation 4.6 varies along the radial direction due to changes in species concentrations along the reacting surface. This blowing velocity opposes the impinging tube flow and causes the establishment of a stagnation plane a small distance above the reacting surface. The species concentrations are updated using the conservation of species at a reacting interface,

$$
\frac{\partial}{\partial t}\left(\rho^{\prime} Y_{i}\right)+\rho Y_{i} v_{n}-\rho D_{i} \frac{\partial Y_{i}}{\partial n}-w_{i}^{\prime}=0
$$

Chapter 2 covers the details of these derived interface conditions, but it is important to note that these boundary conditions allow for multidimensional variations in both fluid 
momentum and species boundary conditions along the surface.

\subsubsection{Optimal Experimental Configuration: Satisfying Quasi One-Dimensional Theory}

The quasi one-dimensional theory offers the ability to conduct parameter studies at computational speeds much faster than multidimensional solvers. In support of this dissertation, a study that focused on the optimal geometric configuration for using the quasi one-dimensional theory through an analysis of separation distances and nozzle radii resulted in a publication that has been accepted for publication in Combustion Science and Technology (special volume for Forman William's $80^{\text {th }}$ Birthday). The paper is attached in Appendix A. In the study, an error metric was used to show that the accuracy of the quasi one-dimensional theory is dependent on separation distance and nozzle exit radius. At short separation distance $(L<4 \mathrm{~mm})$, radial momentum gradients are strong and result in off-ideal behavior of fluid self-similarity. At large separation distances $(L>13 \mathrm{~mm})$, the self-similarity assumption breaks-down as the free-floating limit [68] is approached. The study resulted in the following conclusions:

1. There is an optimal separation distance in small diameter counterflow nozzles.

2. This separation distance corresponds to $L=13 \mathrm{~mm}$ for $13 \mathrm{~mm}$ diameter nozzles. Distances larger than this approached the free-floating regime, whereas distances shorter than this deviated from quasi one-dimensional theory due to radial gradients.

3. An increase in nozzle radius decreased the error metric.

4. The quasi one-dimensional theory is sensitive to all geometric configurations.

Currently no such studies exist detailing the optimal configuration for tube jet flows, which resulted in the work presented in this section. 
Initially, this study assumed a tube radius of $R=10 \mathrm{~mm}$ with a parabolic tube flow boundary condition with $V_{\text {plug }}=0.5 \mathrm{~m} / \mathrm{s}$. As previously stated, a tube length of distance of $2 R, 20 \mathrm{~mm}$, was included to allow separation distance to affect exit velocity profiles. Several reacting flow cases were run at a constant surface temperature of $2000 \mathrm{~K}$ using the porous carbon surface reaction model. The separation distances studied were $L=$ $[4,6,8,10,15,20] \mathrm{mm}$.

Figure 4.4a shows the axial velocity, $v_{z}$, along the axis of symmetry for the $L=8 \mathrm{~mm}$ and $L=15 \mathrm{~mm}$ cases. Accompanying these profiles are the quasi one-dimensional results for the same separation distances and boundary conditions (shown as a dashed, - -, line). The quasi one-dimensional velocity agrees better with the shorter, $L=8 \mathrm{~mm}$, separation distance. Figure 4.4b shows the self similarity function, $U(z)$, for $L=8$ and $L=15 \mathrm{~mm}$ separation distances. Similar to axial velocity, $L=8 \mathrm{~mm}$ is in better agreement with the quasi one-dimensional theory's self-similarity function.

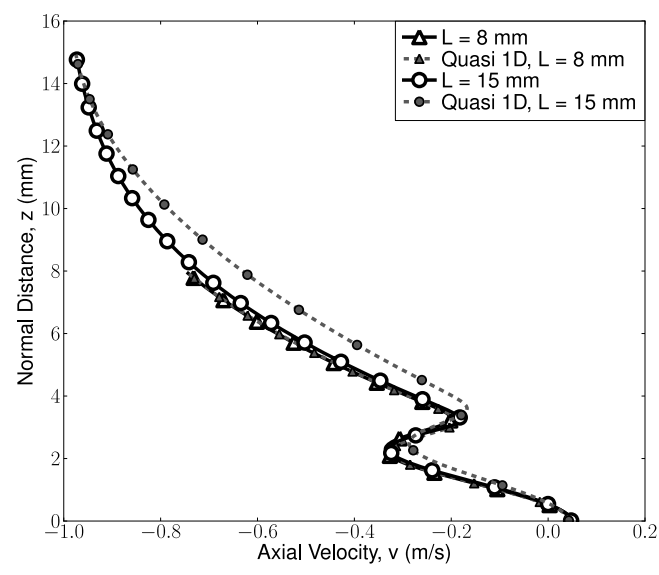

(a) Axial velocity, $v_{z}$, for two separation distances, $L=15 \mathrm{~mm}$ and $L=8 \mathrm{~mm}$

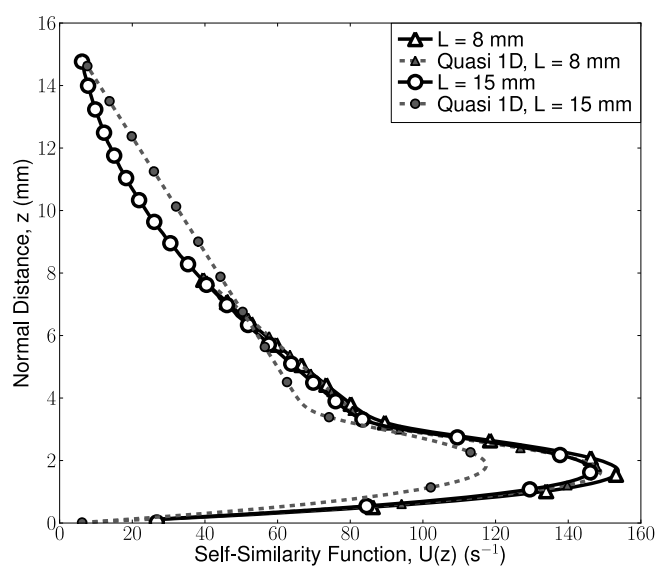

(b) Self similarity function, $U(z)$, for two separation distances, $L=1.5 \mathrm{~cm}$ and $L=$ $0.8 \mathrm{~cm}$

Figure 4.4: Axial velocity, $v_{z}$, and self-similarity function, $U(z)$ for two separation distances.

Figures 4.4a and 4.4b are fine for qualitatively assessing optimal separation distances, however, such observations would not give a concrete assessment of an optimal config- 
uration tube flow configuration. To achieve a quantitative analysis of optimal separation distances several metrics were constructed. The $\mathrm{L}_{2}$-norm,

$$
L_{2} \equiv \sqrt{\frac{1}{N} \sum_{i=1}^{N}\left(\phi_{2 D, i}-\phi_{1 D, i}\right)^{2}}
$$

was used as an error metric for one-to-one comparison between self-similarity, $\phi_{i}=U_{i}$, and pressure radial curvature, $\phi_{i}=\Lambda_{i}=\frac{1}{r} \frac{\partial p}{\partial r}$. Here, the $2 D$ and $1 D$ subscripts denote the two-dimensional and quasi one-dimensional results, respectively, and the $i$ subscript denotes the value at a discrete point for $N$ number of points (discretizations were the same, so point $i$ is at the same location in both simulations).

In addition to the $L_{2}$-norm, it was important to quantify the off-ideal tube exit velocity profile; that is, how the center line varies from the ideal parabolic flow formed in a tube. Such quantification is important because experimentalists will only have volume flow rates and will not be measuring the out-flow velocity profile. The metric $\left(v_{o}-v_{p}\right) / v_{p}$ gives the percent error in the deviation from ideal parabolic flow. Here, $v_{o}$ is the axis of symmetry axial velocity value from the two-dimensional solution and $v_{p}$ is the axis of symmetry velocity for an ideal parabolic tube flow, $v_{p}=2 V_{p l u g}$. Likewise, the lack of experimental velocity measurements leaves the value of $U(z)$ at the tube exit as an unknown. Currently no method exists to approximate $U(z)$ at the boundary without experimental velocity data. In order to use the quasi one-dimensional theory without velocity measurements one would need both $v_{o}=v_{p}$ and $U_{o}=0$. Therefore, both $\left(v_{o}-v_{p}\right) / v_{p}$ and $U(z)$ must be minimized in choosing an optimal separation distance.

The last quantity that was optimized was the height of the $\mathrm{CO}_{2}$ reaction layer thickness, $h_{\mathrm{CO} 2}$. From communications with the researchers at AFIT, it was determined that the experimental resolution is on the order of $0.5 \mathrm{~mm}$. This required the choice of the optimal separation distance not result in a reaction layer too thin to measure using the experimental resolution. The $\mathrm{CO}_{2}$ reaction layer height was determined as the z-direction distance 
where where $Y_{\mathrm{CO}_{2}}=0.0001$ and greater than the normal location of $\mathrm{CO}_{2}$ 's maximum concentration.

Figure 4.5 shows how these quantities are affected by separation distance. For illustration purposes, both the $L_{2}$-norm of $U(z)$ and $\Lambda$ were normalized to the smallest separation distance, $L=4 \mathrm{~mm}$, and the tube exit self-similarity boundary condition, $U_{o}$, was normalized by $100 \mathrm{~s}^{-1}$. The $\mathrm{CO}_{2}$ reaction layer height, $\mathrm{h}_{C O 2}$, is reported in centimeters. The $L_{2}$ norm of $\Lambda, U_{o}$, and $\frac{v_{o}-v_{p}}{v_{p}}$ monotonically decrease with increase in separation distance. The $\mathrm{CO}_{2}$ reaction layer height does not change significantly in terms of experimental resolution, meaning that any of the lengths will yield the same reaction layer thickness. The self-similarity function, $U(z)$, is minimal at $L=8 \mathrm{~mm}$.

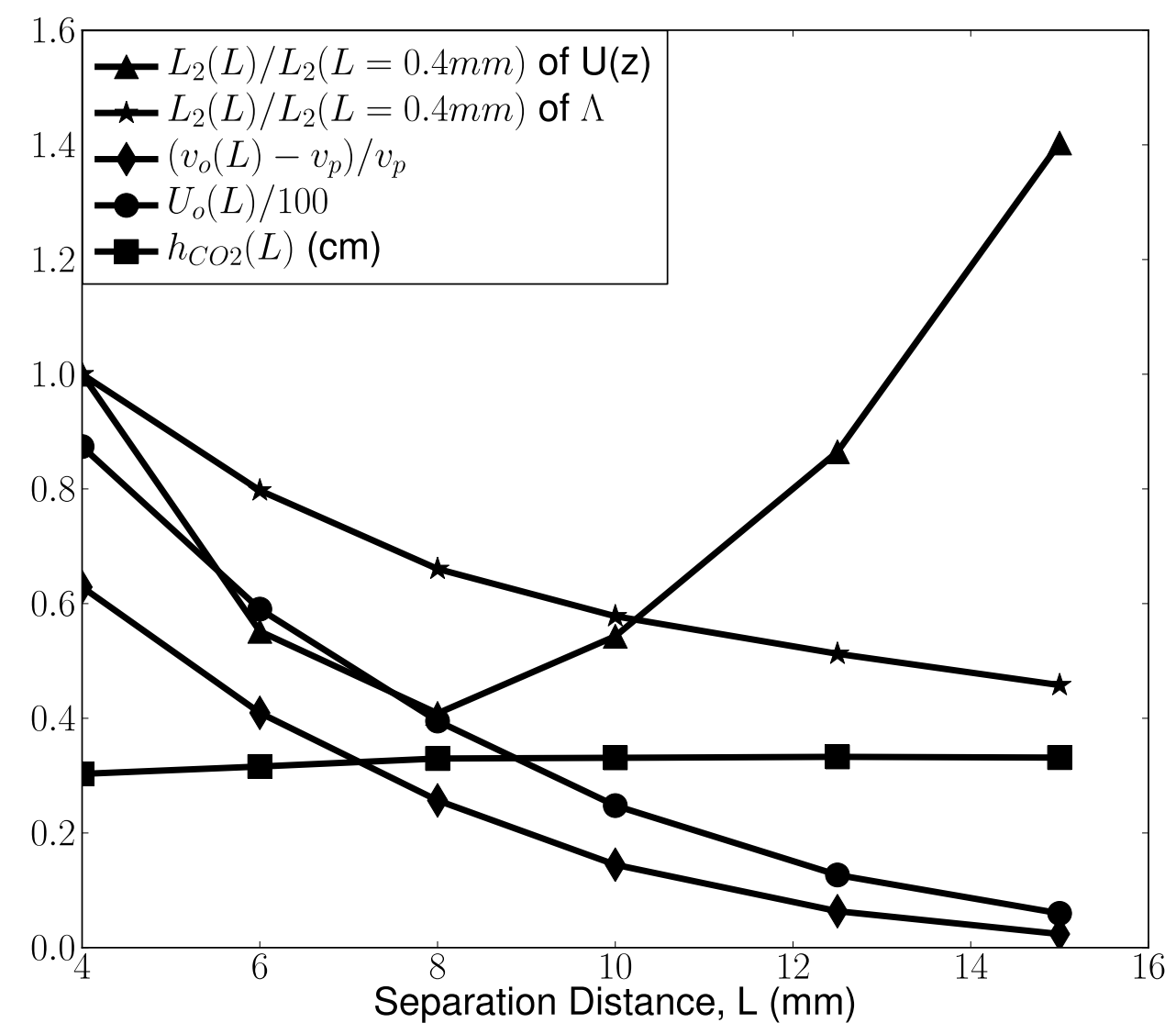

Figure 4.5: Response of various stagnation flow variables as a function of length 
For this specific geometric configuration, the optimal separation distance from this study's assessment of the by self-similarity error metrics is $8 \mathrm{~mm}$. At this separation distance the axial velocity boundary condition differed by 20 percent from ideal parabolic flow and $U_{o}=40 \mathrm{~s}^{-1}$. At larger separation distances, the axial velocity and self similarity boundary conditions did reach their ideal values; however, the self-similarity error rose. This is indicative of approaching the free-floating regime where self-similarity is inapplicable (see Appendix A).

One aspect of these axisymmetric flow configurations that was not explored in previous work was the accuracy of the quasi one-dimensional formulation as a function of tube-jet velocity. Figure 4.6 shows the same error metrics as Figure 4.5 and how they change with increase in tube-jet velocity. The error metrics for the quasi one-dimensional formulation all increased with velocity. Additionally, off-ideal exit boundary conditions, $U_{o} \neq 0$ and $\left|v_{p}-v_{o}\right|$, increased with velocity. 


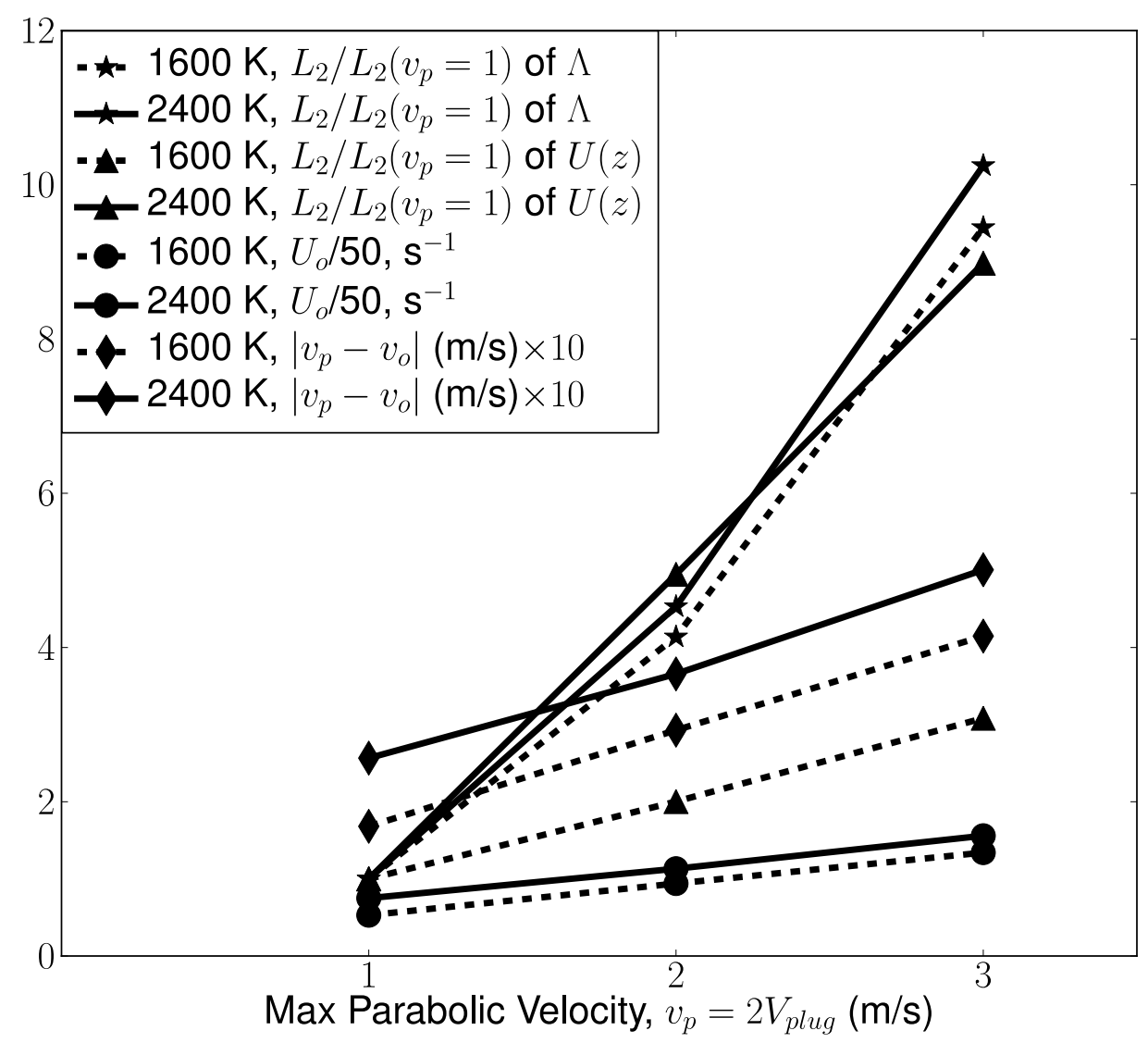

Figure 4.6: Response of various stagnation flow variables as a function of max parabolic velocity.

The effects of separation distance and increased velocity are related. At low separation distances and high flow velocities, the radial curvatures along the axis of symmetry must be large. Additionally, the influence of the stagnation plane, particularly the high pressure, alters the impinging fluid flow. This alteration not only affects boundary conditions to the point where they become off-ideal, but decreases the applicability of flow self-similarity. For lower temperatures (1600 K shown with dashed, --, line) the increase was less. This decrease in error metric is due to the decrease in thermal boundary layer thickness. The larger this thermal layer is, the more it will affect the exit velocity profile non-uniformity. 


\subsection{Results and Discussion}

\subsubsection{Carbon Dioxide Flame Detachment}

With the establishment that the formulation is applicable within certain configuration constraints from the error metric analysis, a quasi one-dimensional code was implemented for further analysis. This allowed for the exploration of how certain response quantities, such as $\mathrm{CO}_{2}$ reaction layer and carbon mass loss rate, changed with flow parameters and surface temperatures. It should be noted that, even though deviations between two dimensional and quasi one-dimensional results increased with increase in velocity, the general trends of $\mathrm{CO}_{2}$ reaction layer thickness were still comparable between the two-dimensional and one-dimensional results. Therefore, the quasi-one dimensional results where velocity was changed must be examined with the caveat that the predictions are general expectations and may or may not support the actual two-dimensional experiment due to the deviations with increased error metrics with increased velocity (see Figure 4.6).

Figure $4.7 \mathrm{a}$ shows the effect of inflow velocity on $\mathrm{CO}_{2}$ flame concentrations over a nonporous reacting surface. These results were generated using the quasi one-dimensional code with fixed surface temperature of $2200 \mathrm{~K}$. There is no sudden extinction of the $\mathrm{CO}_{2}$ flame structure. The $\mathrm{CO}_{2}$ concentration's peak value falls steadily with increase in velocity. The internal graph in Figure 4.7a shows the change in mass loss rate due to this change in velocity, which responds to the $\mathrm{CO}_{2}$ flame presence [26,27]. At velocities greater than 80 $\mathrm{m} / \mathrm{s}$ the mass loss rate ceases to change and $\mathrm{CO}_{2}$ presence is minimal. Such a regime is an interesting region to explore; however, the reaction layers in these high speeds are too small and can not be experimentally worthwhile to investigate with the hyper spectral imaging camera $\left(\mathrm{CO}_{2}\right.$ reacting layer thickness $\left.<0.2 \mathrm{~mm}\right)$. 


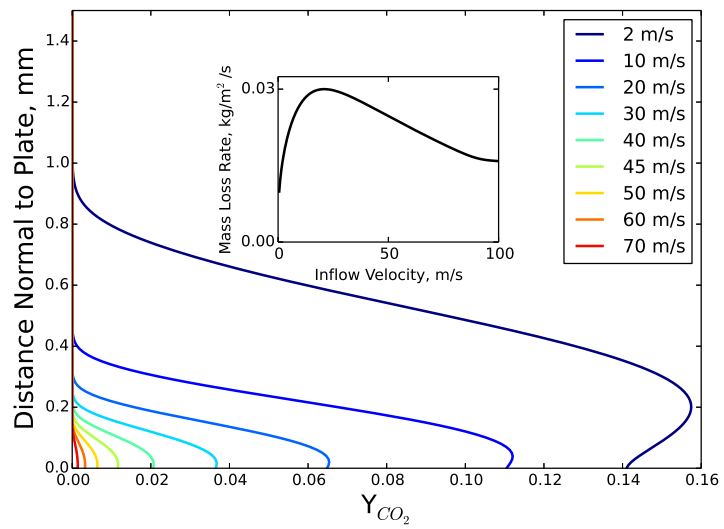

(a) Velocity effects

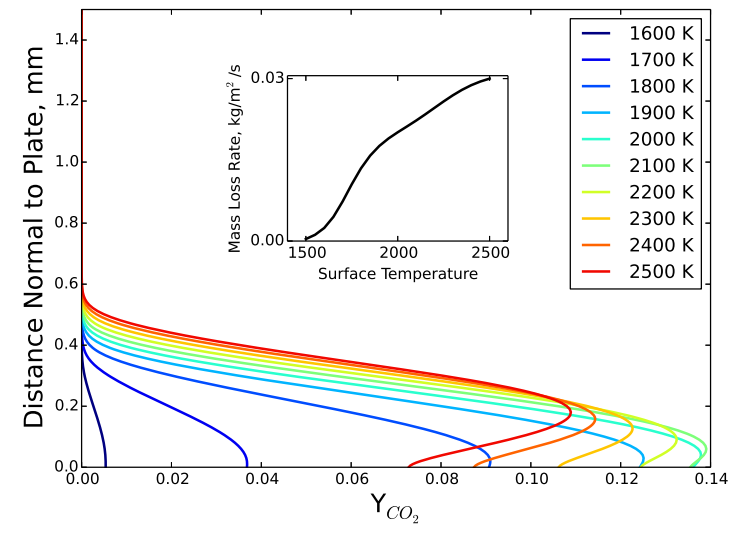

(b) Temperature effects

Figure 4.7: Change in mass loss rate and reaction layer profile of $\mathrm{CO}_{2}$ due to surface velocity, (a), and temperature, (b), effects. Velocity response reported here is for $T_{s}=2200$ $\mathrm{K}$ and nonporous carbon. The temperature response here is for $v_{o}=1 \mathrm{~m} / \mathrm{s}$.

Contrary to flame extinction, $\mathrm{CO}_{2}$ flame detachment-attachment [17-19] is a more accesible regime to experimentally explore using a hyper spectral imaging camera. Figure 4.7b shows $\mathrm{CO}_{2}$ concentrations for a fixed in-flow velocity of $2 \mathrm{~m} / \mathrm{s}$ and varying surface temperature. The $\mathrm{CO}_{2}$ profile is lifted off as surface temperature is increased, which is known as flame detachment. The contrary, flame attachment, is caused by either decreasing temperature (Figure 4.7b) or increasing velocity (illustrated in Figure 4.7a in the transition from $2 \mathrm{~m} / \mathrm{s}$ to $10 \mathrm{~m} / \mathrm{s}$ ). This attachment phenomenon with reaction layers on the order of 3 $\mathrm{mm}$, which is measurable using the given resolution of the hyperspectral imaging camera.

To test that the $\mathrm{CO}_{2}$ flame attachment-detachment could be predicted using the OpenFOAM solver, a uniform, plug-flow $4 \mathrm{~m} / \mathrm{s}$ velocity was imposed and surface temperature was varied. Figures $4.8 \mathrm{a}$ and $4.8 \mathrm{~b}$ show two-dimensional OpenFOAM $\mathrm{CO}_{2}$ mole fraction results with flow directed in the vertical direction (streamlines are shown in white) for a reacting carbon surface with temperatures 1800 and $2000 \mathrm{~K}$ respectively. 


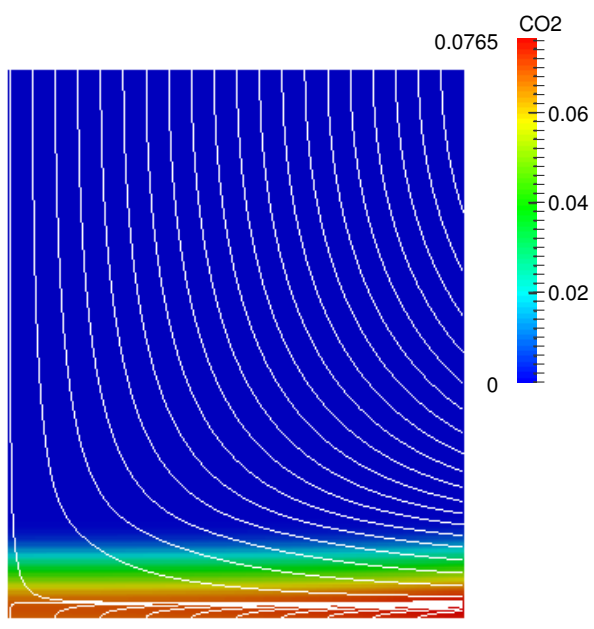

(a) $T_{s}=1800 \mathrm{~K}$

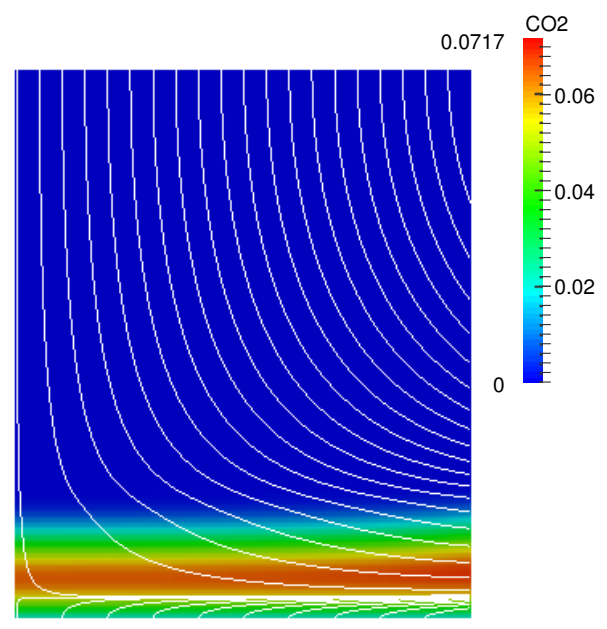

(b) $T_{s}=2000 \mathrm{~K}$

Figure 4.8: Two dimensional stagnation results for porous carbon model with plug-flow velocity of $3 \mathrm{~m} / \mathrm{s}$. Contour colors show $\mathrm{CO}_{2}$ mole fraction profiles and white lines are stream-lines.

The higher temperature causes the $\mathrm{CO}_{2}$ reacting layer to detatch and higher concentrations of $\mathrm{CO}_{2}$ are found in the radial directions. The results section will report how the reacting structures and carbon mass loss rates are affected by inflow velocity profiles caused by the experimental geometric configuration.

\section{Response to Experimental Conditions}

Conversations with the AFIT experimental collaborators made it apparent that large parameter sweeps were a necessity in order to examine flow condition effects. This section briefly touches on these studies, namely the effects of surface temperature coupled with humidity and in-flow velocity. Information gathered by these parametric studies gave insight to the most sensitive parameters in the stagnation flow configuration and helped construct the conditions for the experiments and two-dimensional simulations.

The results discussed here address $\mathrm{CO}_{2}$ flame thickness, which is defined as the normal distance height (z-direction) where $Y_{\mathrm{CO} 2}$ fell below 0.001 after its local maximum. Figure 
4.9a shows the thickness response to far-field velocity, $v_{\infty}=v_{o}$, and surface temperature $T_{s}$. At low velocities and high temperatures the reaction layer height is the largest.

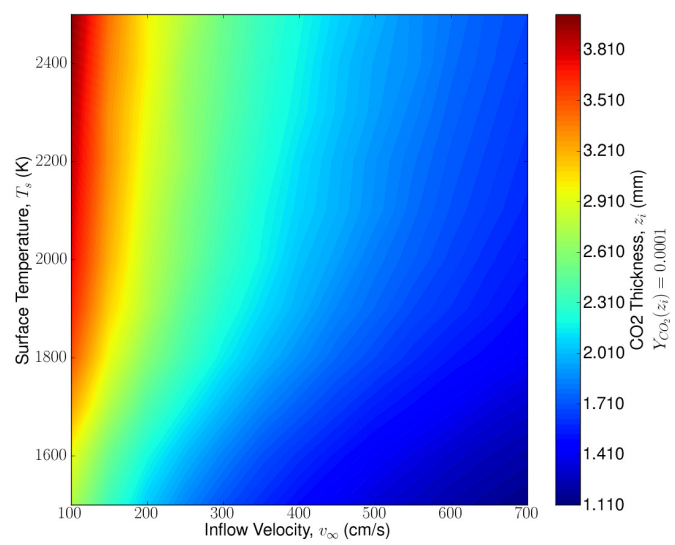

(a) Variation of surface temperature and velocity, $v_{o}$, and their effect on $\mathrm{CO}_{2}$ reaction layer thickness.

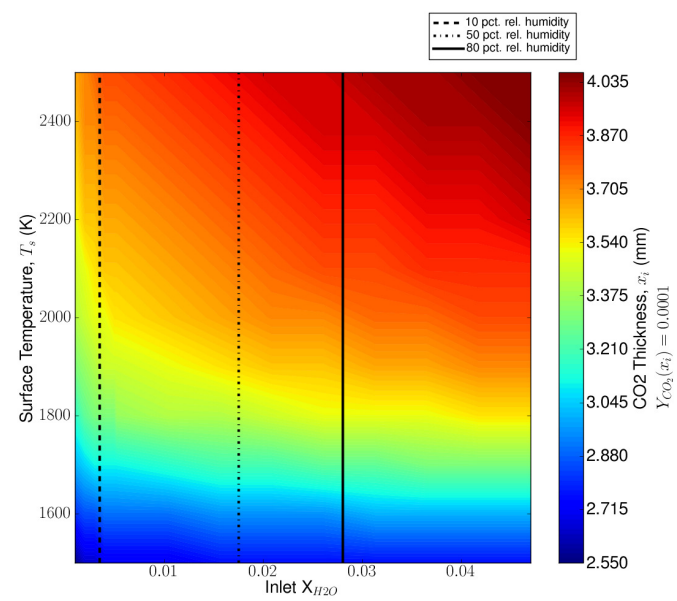

(b) Variation of surface temperature and relative humidity and their effect on $\mathrm{CO}_{2}$ reaction layer thickness.

Figure 4.9: Response of $\mathrm{CO}_{2}$ reaction layer height to two-dimensional parameter sweeps.

Additionally, Figure $4.9 \mathrm{~b}$ shows the response of reaction layer height to relative humidity effects. Experiments will take place in New Mexico, where the dry climate typically ${ }^{2}$ has relative humidity values on the order of $10 \%$. The conversion of $\mathrm{CO}$ to $\mathrm{CO}_{2}$ is rate controlled by the $\mathrm{H}_{2} \mathrm{O}$ presence in the gaseous phase; $\mathrm{CO}+\mathrm{OH}$ and $\mathrm{CO}+\mathrm{H}$ reactions are driving mechanisms for $\mathrm{CO}$ oxidation. These humidity results are reassuring, as low humidity (10 $\%$ relative humidity shown with dashed, --, line) produces reaction layer thicknesses that are still experimentally measurable.

\subsubsection{Two-Dimensional Effects}

With the guidance of the quasi one-dimensional formulation, an array of two-dimensional simulations were performed in order to predict multidimensional effects that could be observed experimentally. To study temperature effects, the the parabolic velocity profile was held constant, with $V_{\text {plug }}=0.5 \rightarrow v_{p}=1 \mathrm{~m} / \mathrm{s}$, for a range of surface temperatures,

\footnotetext{
${ }^{2}$ This is from private communication with AFIT
} 
$T_{s}=[1600,1800,2000,2200,2400] \mathrm{K}$. The nonporous and porous models were used to analyze the effect that the different surface reaction rates have on the reaction structures.

Figures 4.10 and 4.11 show the mole fraction results for $\mathrm{CO}, \mathrm{CO}_{2}, \mathrm{O}_{2}, \mathrm{H}_{2} \mathrm{O}, \mathrm{O}$, and $\mathrm{OH}$ for the simulations using the nonporous reaction model at $T_{s}=1600$ and $2400 \mathrm{~K}$, respectively. $T_{s}=[1800,2000,2200] \mathrm{K}$ were omitted for brevity. The increase in temperature and subsequent increase in surface reactivity causes a stronger presence of carbon monoxide in the $T_{S}=2400 \mathrm{~K}$ case. $\mathrm{CO}_{2}$ and $\mathrm{OH}$ are attached in the $T_{s}=1600 \mathrm{~K}$ case and are detached in the higher temperature, $T_{s}=2400 \mathrm{~K}$, case. All species structures exhibit a widening in reaction layer thickness in the radial direction. This is due to the decrease in axial momentum velocity in the radial direction from the parabolic-like axial velocity profile at the tube exit. At the higher temperature, the maximum $\mathrm{CO}_{2}$ concentration decrease due to larger consumption rates at the surface.

Figure 4.12 and 4.13 show the mole fraction results for $\mathrm{CO}, \mathrm{CO}_{2}, \mathrm{O}_{2}, \mathrm{H}_{2} \mathrm{O}, \mathrm{O}$, and $\mathrm{OH}$ for the simulations using the porous reaction model at $T_{s}=1600$ and $2400 \mathrm{~K}$, respectively. These cases behave similarly to the nonporous case because the reaction structures widen due to decrease in axial velocity in the radial direction. $\mathrm{O}$ and $\mathrm{OH}$ species concentrations are in attached mode at the lower temperature, which is the main notable difference between the two nonporous and porous models. The porous model reaction rates are much higher (see Chapter 2, Section 2.5.3, and the results from Chapter 3) which causes a full order of magnitude higher maximum concentration of carbon monoxide in comparison to the nonporous model at $T_{s}=1600 \mathrm{~K}$. 

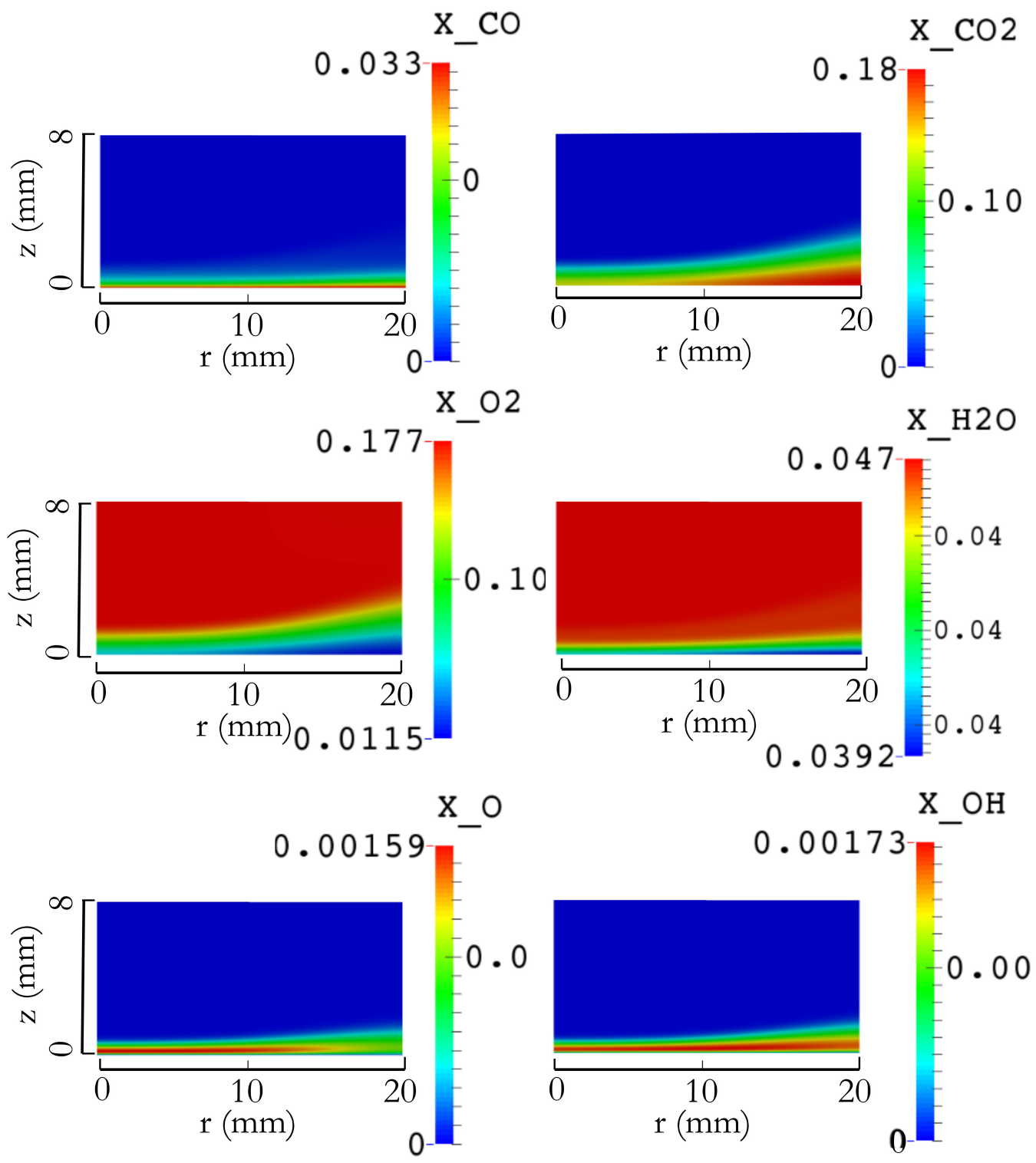

Figure 4.10: Mole fraction results for $\mathrm{CO}, \mathrm{CO}_{2}, \mathrm{O}_{2}, \mathrm{H}_{2} \mathrm{O}$, O, and $\mathrm{OH}$ for the simulations using the nonporous reaction model at $T_{s}=1600 \mathrm{~K}$. . The tube region included in the simulation is not shown in these images as no reactions occurred within the tube walls. 

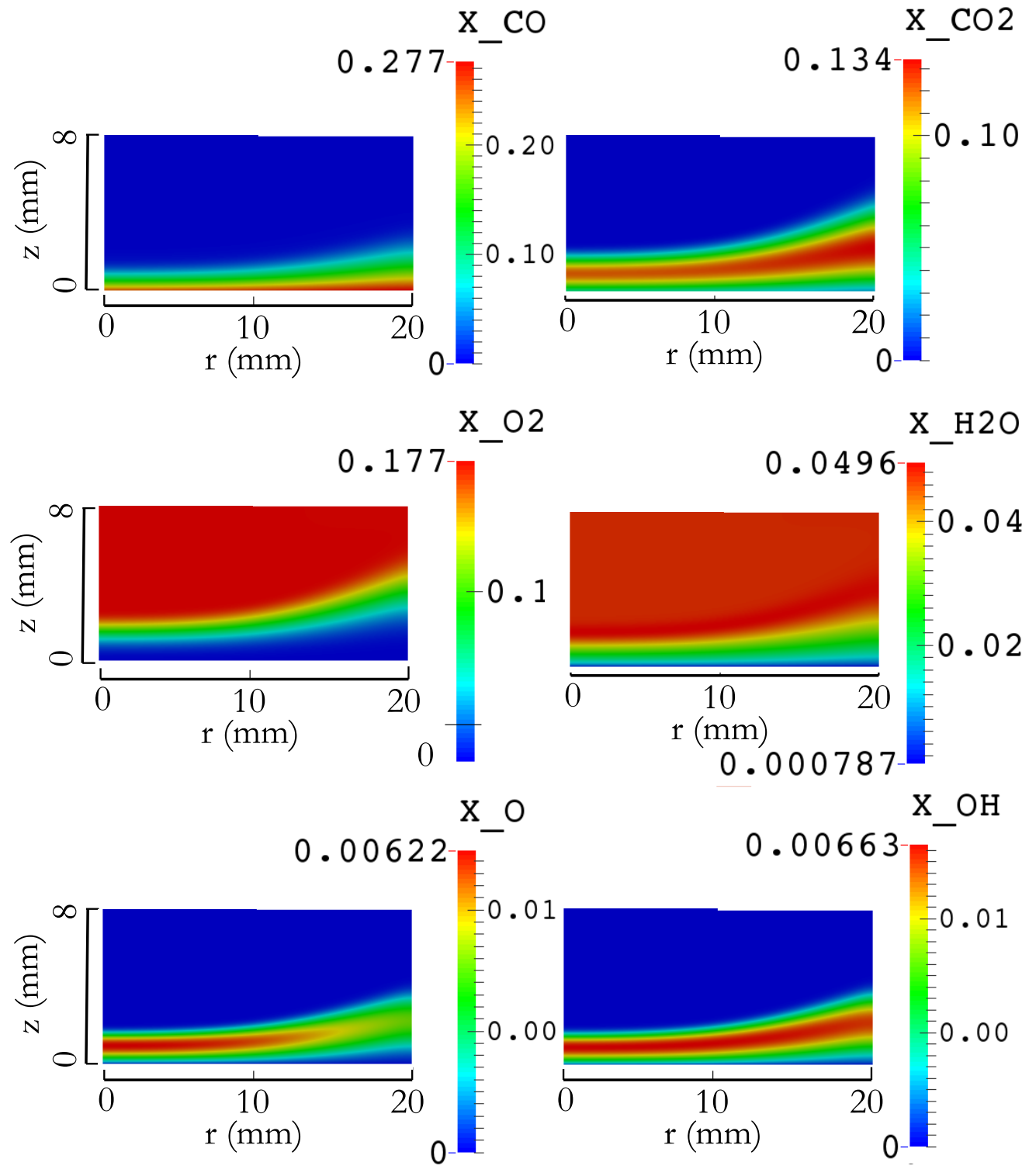

Figure 4.11: Mole fraction results for $\mathrm{CO}, \mathrm{CO}_{2}, \mathrm{O}_{2}, \mathrm{H}_{2} \mathrm{O}, \mathrm{O}$, and $\mathrm{OH}$ for the simulations using the nonporous reaction model at $T_{s}=2400 \mathrm{~K}$. . The tube region included in the simulation is not shown in these images as no reactions occurred within the tube walls. 

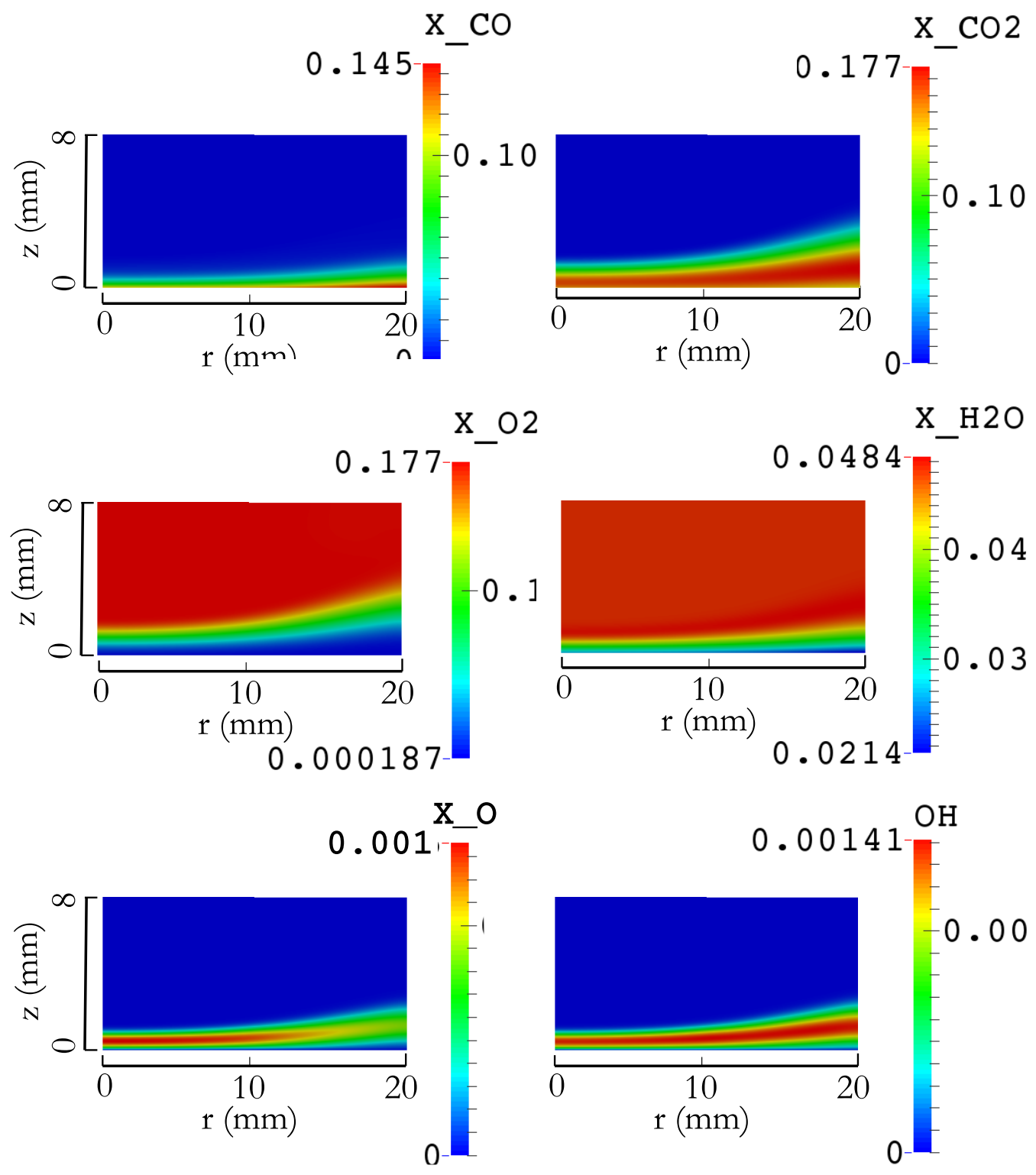

Figure 4.12: Mole fraction results for $\mathrm{CO}, \mathrm{CO}_{2}, \mathrm{O}_{2}, \mathrm{H}_{2} \mathrm{O}, \mathrm{O}$, and $\mathrm{OH}$ for the simulations using the porous reaction model at $T_{s}=1600 \mathrm{~K}$. . The tube region included in the simulation is not shown in these images as no reactions occurred within the tube walls. 

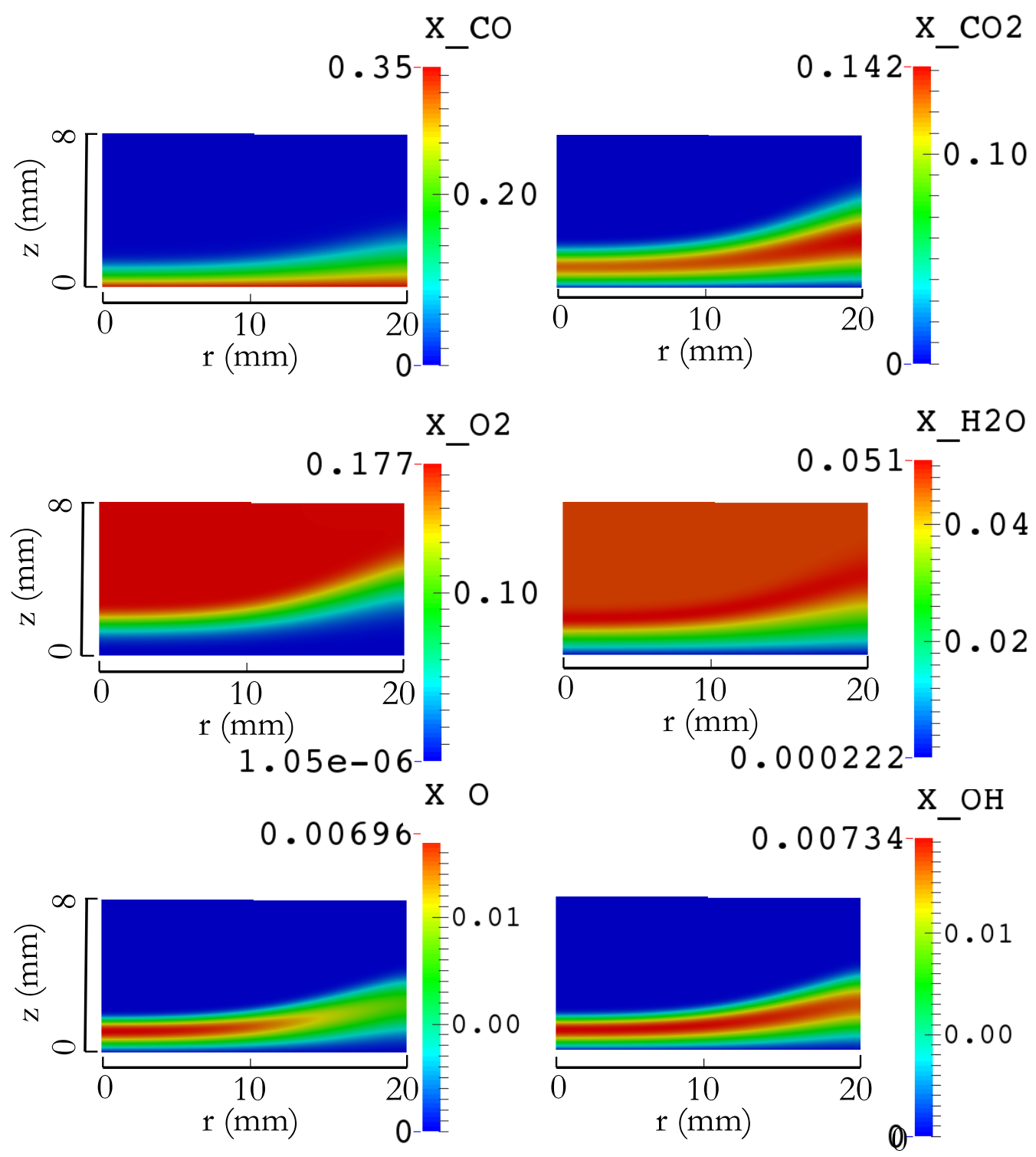

Figure 4.13: Mole fraction results for $\mathrm{CO}, \mathrm{CO}_{2}, \mathrm{O}_{2}, \mathrm{H}_{2} \mathrm{O}, \mathrm{O}$, and $\mathrm{OH}$ for the simulations using the porous reaction model at $T_{s}=2400 \mathrm{~K}$. . The tube region included in the simulation is not shown in these images as no reactions occurred within the tube walls. 
Figures 4.14 and 4.15 show the concentration profiles as a function of normal distance for surface temperatures $T_{s}=[1600,2400] \mathrm{K}$, for nonporous and porous models, respectively. The profiles are extracted at two locations, $r=0$ and $r=10 \mathrm{~mm}$.

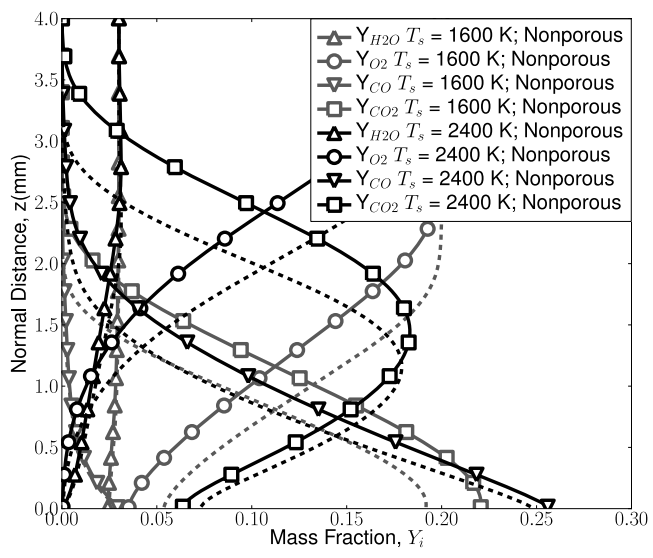

(a) Species: $\mathrm{H}_{2} \mathrm{O}, \mathrm{CO}_{2}, \mathrm{CO}$, and $\mathrm{O}_{2}$

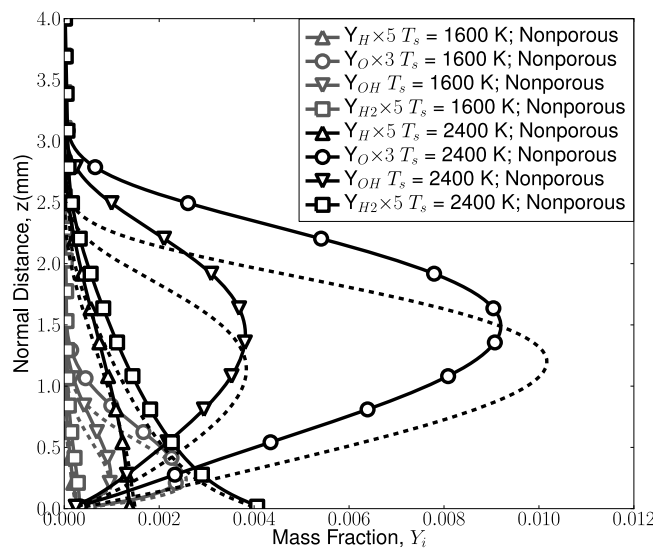

(b) Species: $\mathrm{H}, \mathrm{OH}, \mathrm{O}, \mathrm{H}_{2}$

Figure 4.14: Species concentration results as function of normal distance, $\mathrm{z}(\mathrm{mm})$, for $T_{s}=[1600,2400] \mathrm{K}$ two locations, $r=0$ (dashed lines) and $r=10 \mathrm{~mm}$ (solid lines), using the nonporous carbon surface model.

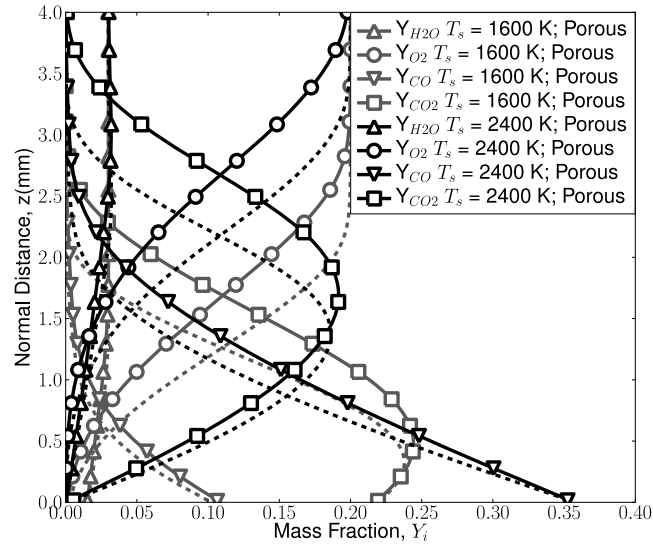

(a) Species: $\mathrm{H}_{2} \mathrm{O}, \mathrm{CO}_{2}, \mathrm{CO}$, and $\mathrm{O}_{2}$

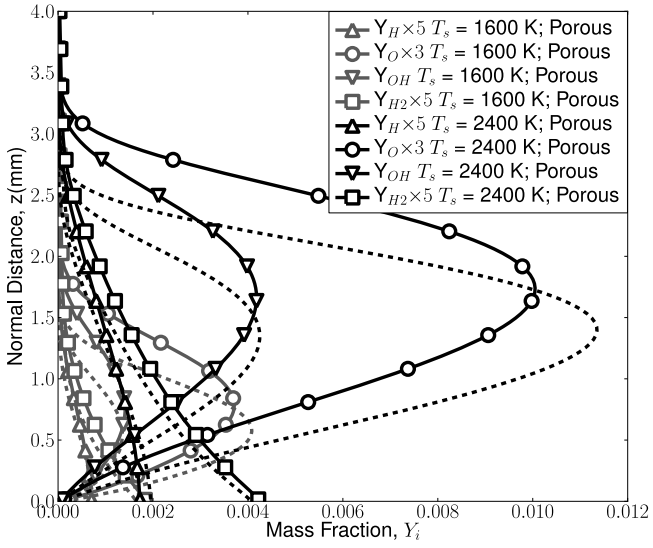

(b) Species: $\mathrm{H}, \mathrm{OH}, \mathrm{O}, \mathrm{H}_{2}$

Figure 4.15: Species concentration results as function of normal distance, $\mathrm{z}(\mathrm{mm})$, for $T_{s}=[1600,2400] \mathrm{K}$ two locations, $r=0$ (dashed lines) and $r=10 \mathrm{~mm}$ (solid lines), using the porous carbon surface model.

For all models, the reaction layers thicken in the radial direction. This widening is 
also a function of temperature. The $T_{s}=1600 \mathrm{~K}$ case for both nonporous and porous surface reaction models and the $T_{s}=2400 \mathrm{~K}$ case for the nonporous model have reaction layer concentrations that increase by less than $5 \% \mathrm{~mm}$. However, the nonporous $T_{s}=$ $1600 \mathrm{~K}$ case does present an increase in species concentration in the radial direction; in the case of $\mathrm{CO}_{2}$ the increase is on the order of $20 \%$. A low temperature experiment with nonporous carbon surfaces will produce measurable increases in concentrations through proper calibration. Thus, it is conceivable that such an experimental trend can be observed through proper calibration.

The $T_{s}=1600 \mathrm{~K}$ solutions have attached reacting structures for both models. The higher temperature cases, $T_{s}=2400 \mathrm{~K}$, have detached structures for both surface models. The detachment by increasing temperature from $T_{s}=1600 \mathrm{~K}$ to $T_{s}=2400 \mathrm{~K}$ yields reacting layers that differ in concentration, for example $\mathrm{OH}$ concentration is nearly doubled in both cases. Additionally, the reaction layer height increases. For example, the $\mathrm{CO}_{2}$ reaction layers increase by $1 \mathrm{~mm}$ as well as change in structure (monotonic at low temperatures, non-monotonic at high temperatures). These trends are experimentally measurable with an image resolution of $<1 \mathrm{~mm}$ that is capable of capturing changes of $10 \%$ in concentration.

The radial widening of the reaction layers in the porous $T_{s}=2400 \mathrm{~K}$ case is on the order of $1 \mathrm{~mm}$, which is experimentally detectable. Unlike the other cases, the concentrations do not increase in the radial direction in this high temperature porous case. This is due to both the widening (more area for reactants to cover) and increase in surface and gas-phase reactivity that consumes the species. Table 4.2 summarizes these results and the experimental considerations that are necessary to measure these temperature surface porosity dependencies. 
Table 4.2: Table of experimental considerations for porous and nonporous surfaces with $T_{s}=[1600,2400] \mathrm{K}$ surface temperatures.

\begin{tabular}{|c|c|c|}
\hline Case & Simulation Observation & Experimental Considerations \\
\hline \multirow[t]{3}{*}{$T_{s}=1600 \mathrm{~K} ;$ Nonporous } & Widening of reaction layer & Need $<0.5 \mathrm{~mm}$ resolution \\
\hline & Increase in concentration & Calibrate to see $20 \%$ change \\
\hline & Attached reaction profiles & $\begin{array}{l}\text { Six data points at resolution of } 0.5 \\
\text { mm. Calibrate to see } 10 \% \text { change be- } \\
\text { tween points }\end{array}$ \\
\hline \multirow[t]{3}{*}{$T_{s}=2400 \mathrm{~K} ;$ Nonporous } & Widening of reaction layer & Need $<0.5 \mathrm{~mm}$ resolution \\
\hline & Increase in concentration & $\begin{array}{l}\text { Small changes in simulation, on order } \\
\text { of } 5 \%\end{array}$ \\
\hline & Detached reaction profiles & $\begin{array}{l}\text { Seven data points at resolution of } 0.5 \\
\text { mm. Calibrate to see } 10 \% \text { change be- } \\
\text { tween points }\end{array}$ \\
\hline \multirow[t]{3}{*}{$T_{s}=1600 \mathrm{~K} ;$ Porous } & Widening of reaction layer & Need $<0.5 \mathrm{~mm}$ resolution \\
\hline & Increase in concentration & $\begin{array}{l}\text { Small changes in simulation, on order } \\
\text { of } 5 \%\end{array}$ \\
\hline & Attached reaction profiles & $\begin{array}{l}\text { Five data points at resolution of } \\
0.5 \mathrm{~mm}\end{array}$ \\
\hline \multirow[t]{3}{*}{$T_{s}=2400 \mathrm{~K} ;$ Porous } & Widening of reaction layer & Need $<1 \mathrm{~mm}$ resolution \\
\hline & Increase in concentration & $\begin{array}{l}\text { Small changes in simulation, on order } \\
\text { of } 5 \%\end{array}$ \\
\hline & Detached reaction profiles & $\begin{array}{l}\text { Eight data points with } 0.5 \mathrm{~mm} \text { resolu- } \\
\text { tion }\end{array}$ \\
\hline
\end{tabular}


Figures 4.16 and 4.17 show the surface mass fractions for the species consumed through the heterogeneous reactions along the radial direction. The surface concentrations are dependent not only on temperature but on the surface reaction model. $\mathrm{OH}$ and $\mathrm{O}$ concentrations increase and then fall as temperature is increased in the nonporous mode, but in the porous model the behavior is opposite, fall and then rising with temperature. This transition happens at $T_{s}=2000 \mathrm{~K}$ for both models. $\mathrm{H}_{2} \mathrm{O}$ and $\mathrm{O}_{2}$ fall in concentration with increase in temperature for both models. The stronger reactivity in the porous model causes a more rapid reduction of $\mathrm{O}_{2}$ and $\mathrm{H}_{2} \mathrm{O}$ with increase in temperature.

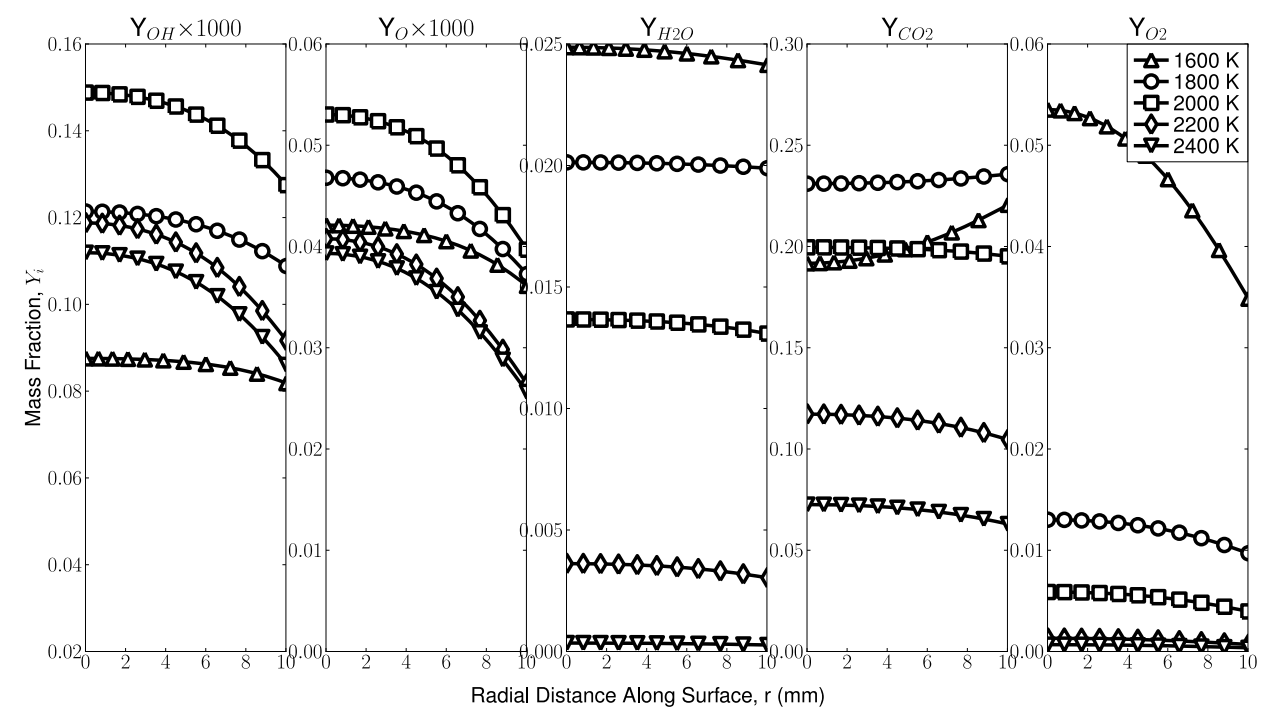

Figure 4.16: Nonporous model consumed surface species mass fractions for $T_{s}=$ $[1600,1800,2000,2200,2400] \mathrm{K}$ and $v_{p}=1 \mathrm{~m} / \mathrm{s}$. 


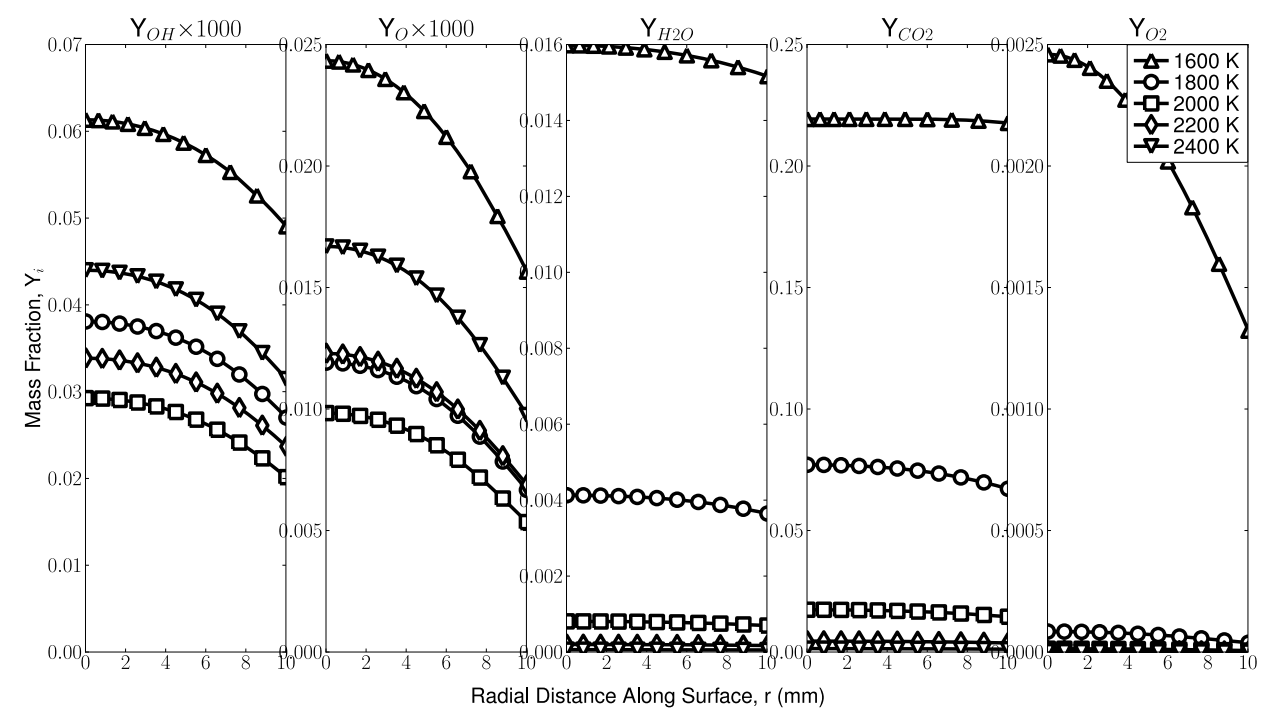

Figure 4.17: Porous model consumed surface species mass fractions for $T_{s}=$ $[1600,1800,2000,2200,2400] \mathrm{K}$ and $v_{p}=1 \mathrm{~m} / \mathrm{s}$.

The amount of $\mathrm{O}_{2}$ variation along the surface is not as prevalent as found in the flat plate results presented in Chapter 3. This is due to how air is delivered to the surface. In the flat plate case, $\mathrm{O}_{2}$ is consumed at the front end of the plate and is not replenished in the down stream section. In the stagnation flow case, oxygen is continuously delivered to the plate (flow is normal). The diffusion and convection directions are both parallel to each other leading to less variation along the plate. In the flat plate scenario, the diffusion and convection directions are orthogonal to each other and diffusion is the dominant transport mechanism for delivering $\mathrm{O} 2$ to the flat plate surface.

The balance between the terms of the $\mathrm{CO}_{2}$ species equation is examined in Chapter 3 with emphasis on residence time. Here the same terms are examined but with emphasis on how they respond to surface temperature. In this section the terms are examined in the normal direction from the plate at surface temperatures $T_{s}=[1600,1800,2000] \mathrm{K}$ and at radial locations $r=0$ (axis of symmetry) and $10 \mathrm{~mm}$; the terms are shown in Figure 4.18. Einstein notation is used to denote the convective, $\rho v_{j} \partial Y_{C O_{2}} / \partial x_{j}$, diffusive, $\partial \rho Y_{\mathrm{CO}_{2}} V_{C O_{2}, j} / \partial x_{j}$, and mass production, $w_{C O_{2}}$, terms. Specifically these conditions are 
examined in order to analyze the transition from attached to detached $\mathrm{CO}_{2}$ flame.

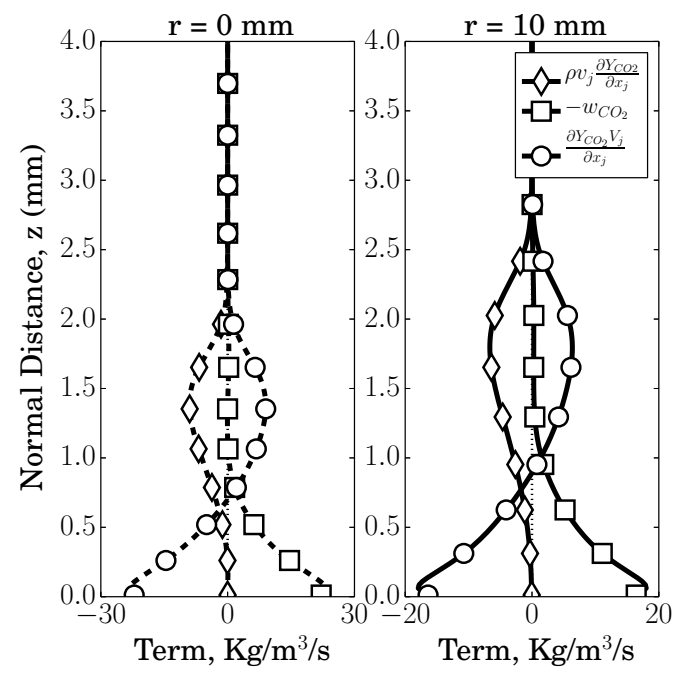

(a) $T_{s}=1600 \mathrm{~K}$

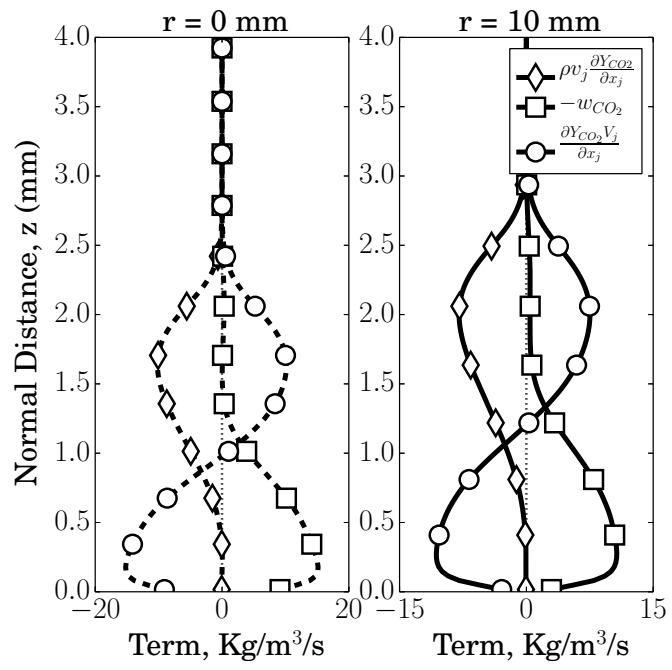

(b) $T_{s}=1800 \mathrm{~K}$

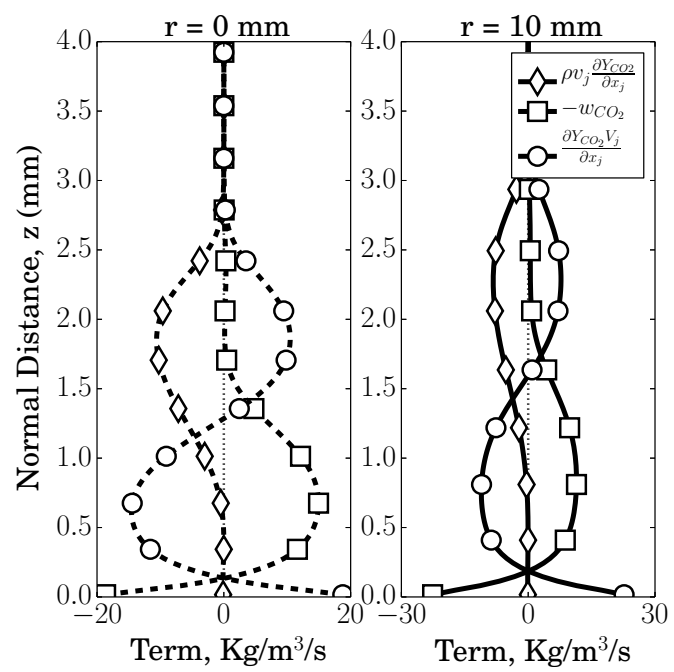

(c) $T_{s}=2000 \mathrm{~K}$

Figure 4.18: Terms of the $\mathrm{CO}_{2}$ species conservation equation, convective (shown with $\left.-\diamond-\right)$, chemical source ( shown with - $\square$-), and diffusion (shown with -o-). Data is sample normal to the plate at locations $\mathrm{r}=0 \mathrm{~mm}$ (shown with dashed line, --) and $\mathrm{r}=10 \mathrm{~mm}$ (shown with solid line, -)

For the $1600 \mathrm{~K}$ case at the surface, Figure 4.18a, the diffusion term is balanced (same in magnitude opposite in sign) with the chemical source term. As temperature is increased to $1800 \mathrm{~K}$, Figure $4.18 \mathrm{~b}$, the terms stay balanced with a reduction in magnitude. Somewhere between $1800 \mathrm{~K}$ and $2000 \mathrm{~K}$ the terms cross each other at the zero line (shown as a dotted 
line) and the signs of the diffusion and chemical terms become opposite of the $1600 \mathrm{~K}$ case. At this crossing the $\mathrm{CO}_{2}$ flame noticeably transitions from an attached to a detached (see 4.4.1).

At the radial location $r=10 \mathrm{~mm}$ the diffusion and chemical source terms are closer to zero than at the $r=0 \mathrm{~mm}$ location for the $T_{s}=1600$ and $1800 \mathrm{~K}$ case. At $T_{s}=2000 \mathrm{~K}$ the terms have changed sign and are farther apart. To explore if a solution exists where the $\mathrm{CO}_{2}$ flame transitions from attached to detached, another case was run with surface temperature $T_{s}=1850 \mathrm{~K}$. Figure 4.19 shows the $\mathrm{CO}_{2}$ species conservation terms, Figure $4.19 \mathrm{a}$, and $\mathrm{CO}_{2}$ reaction layer profile, Figure $4.19 \mathrm{~b}$, for $T_{s}=1850 \mathrm{~K}$. The chemical source and diffusion terms change in sign from the axis of symmetry, $r=0 \mathrm{~mm}$, to the radial location of $r=$ $10 \mathrm{~mm}$, see Figure 4.19a. This causes a transition from attached to detached flame along the surface; the transition is noticeable by carefully considering the monotony at $r=0 \mathrm{~mm}$ and the non-monotony at $r=10 \mathrm{~mm}$ of the $\mathrm{CO}_{2}$ reaction profile in Figure $4.19 \mathrm{~b}$. This confirms that a multidimensional flame attachment-detachment does exist. 


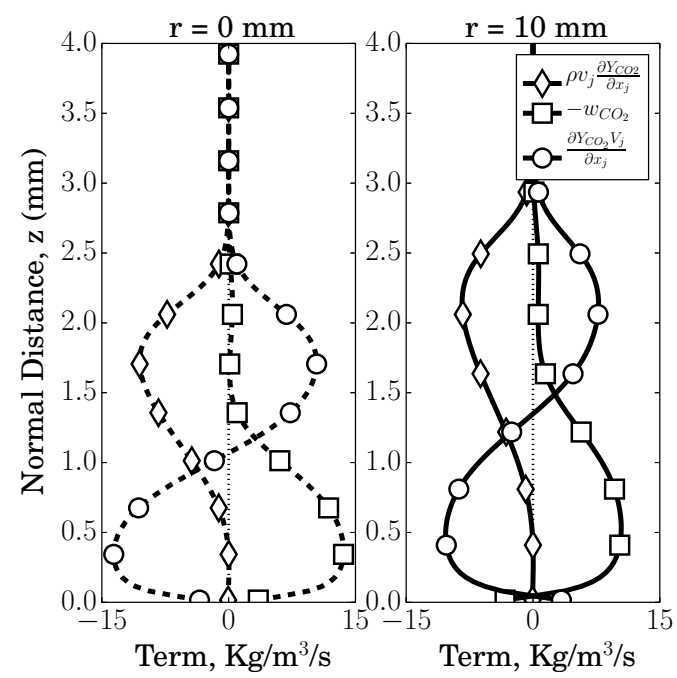

(a) $\mathrm{CO}_{2}$ species conservation equation terms for $T_{s}=1850 \mathrm{~K}$

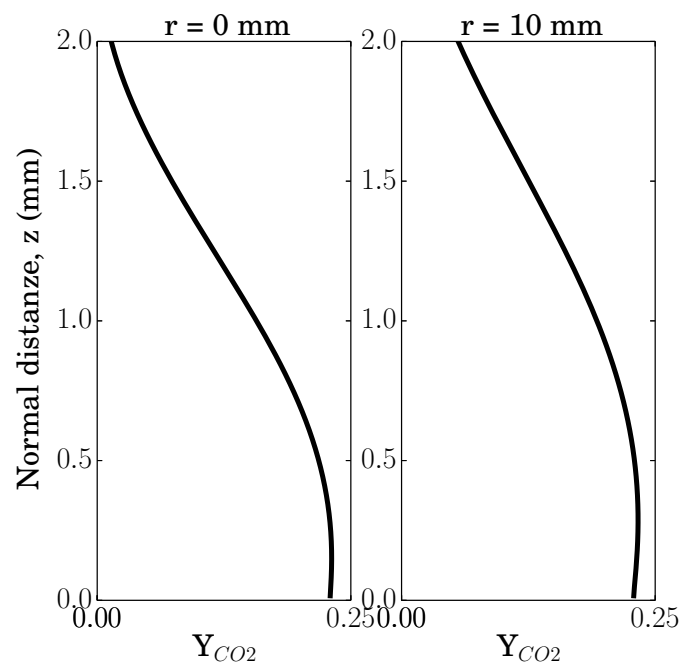

(b) $\mathrm{CO}_{2}$ reaction layer at two different radial locations, $r=10$ and $r=10 \mathrm{~mm}$.

Figure 4.19: Terms of the $\mathrm{CO}_{2}$ species conservation equation, convective (shown with $-\diamond-$ ), chemical source ( shown with - $\square$-), and diffusion (shown with-o-) for $T_{s}=1850 \mathrm{~K}$. Data is sampled normal to the plate at locations $\mathrm{r}=0 \mathrm{~mm}$ (shown with dashed line, --) and $\mathrm{r}=10$ $\mathrm{mm}$ (shown with solid line, -). Also shown is $\mathrm{CO}_{2}$ reaction layer at the two separate radial locations, $r=0$ and $10 \mathrm{~mm}$. At $r=0 \mathrm{~mm}$ the flame is attached and at the $r=10 \mathrm{~mm}$ the flame is detached.

Figure 4.20 shows the mass loss rate as a function of radial distance for $T_{s}=[1600$, $1800,2000,2200,2400] \mathrm{K}$ for both nonporous and porous cases. As the carbon surface reacts, solid carbon is converted into carbon monoxide which causes solid mass to be lost and consequently the surface recesses. For both nonporous and porous cases, the mass loss rate increases with increase in temperature and is multidimensional. The mass loss rate for the nonporous case has distinct profiles for each temperature, with values no less than 10 percent percent of each other. The porous model, however, behaves asymptotically with increase in temperature. The mass loss rate for the porous model cases are within three percent of each other for temperatures of [2000, 2200, 2400] K. This trend of porous carbon mass loss rate behaving asymptotically at higher temperatures and nonporous carbon surfaces consistently changes can be experimentally validated through video measurement of the surface recession rate. This implies that as $T_{s} \rightarrow 2400 \mathrm{~K}$, that surface reactions are 
not rate controlling and the mass loss rate is primarily controlled by diffusion.

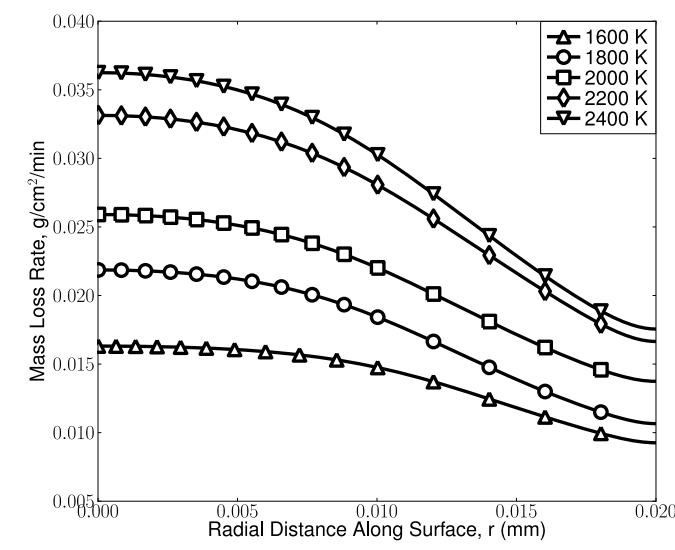

(a) Nonporous mass loss rate

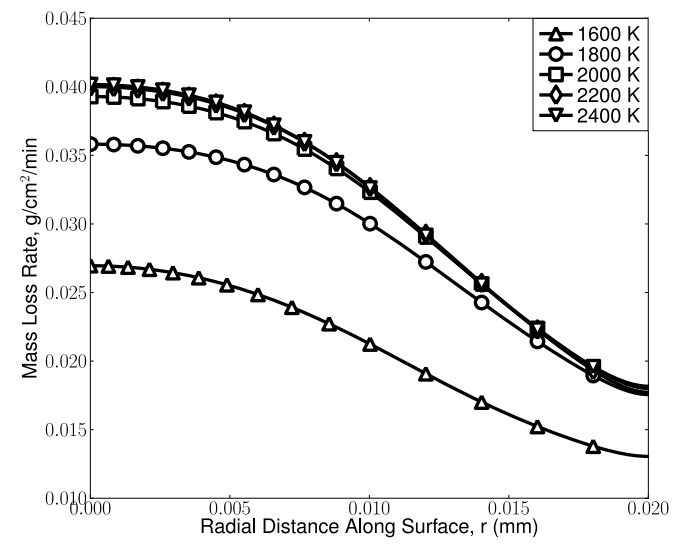

(b) Porous mass loss rate

Figure 4.20: Mass loss rate for $T_{s}=[1600,1800,2000,2200,2400] \mathrm{K}$ for both porous and nonporous models.

\subsubsection{Heterogeneous and Homogeneous Coupling}

As introduced in Chapter 3, the homogeneous coupling for the stagnation configuration is extracted by

$$
\Phi_{k}=\frac{\nu_{k} \frac{W_{C O}}{W_{k}} s_{k}^{\prime}}{w_{C O}^{\prime}} .
$$

Equation 4.9 gives a proportion that a gaseous species, $k$, contributes to the production of carbon monoxide. Figures $4.21 \mathrm{a}$ and $4.21 \mathrm{~b}$ show $\Phi_{k}$ for each reacting species, $\mathrm{k}$, at surface temperatures $T_{s}=[1600,1800,2000,2200,2400] \mathrm{K}$ for the porous and nonporous case respectively. The general behavior of these proportions coincides with the flat plate results as discussed in Chapter 3. In particular, the $\mathrm{CO}_{2}$ reaction dominates the production of $\mathrm{CO}$ in the porous model, whereas, in the nonporous model, minor species, $\mathrm{O}$ and $\mathrm{OH}$, contribute on the order of 40 to 50 percent of $\mathrm{CO}$ production and $\mathrm{CO}_{2}$ is less than one percent of overall CO production. This trend is similar to the homogeneous-heterogeneous coupling found for the flat plate configuration in Chapter 3.

Interestingly, $\mathrm{O}_{2}$ in both cases does not contribute as much to the production of $\mathrm{CO}$ as 
in the flat plate case. The reason for this is that the residence time affects the location of $\mathrm{CO}_{2}$ production less in this scenario. In the flat plate configuration, the direction of the flow produces $\mathrm{CO}_{2}$ in the downstream direction of the plate which caused $\mathrm{O}_{2}$ to contribute more than 60 percent of $\mathrm{CO}$ in the leading edge of the plate. In this stagnation flow scenario, flow is normal to the plate and the $\mathrm{CO}_{2}$ flame is well established over the reacting carbon surface. The direction of the flow and the nature of the $\mathrm{CO}_{2}$ flame front causes $\mathrm{O}_{2}$ to be consumed within the reacting layer more so than in the flat plate scenario.

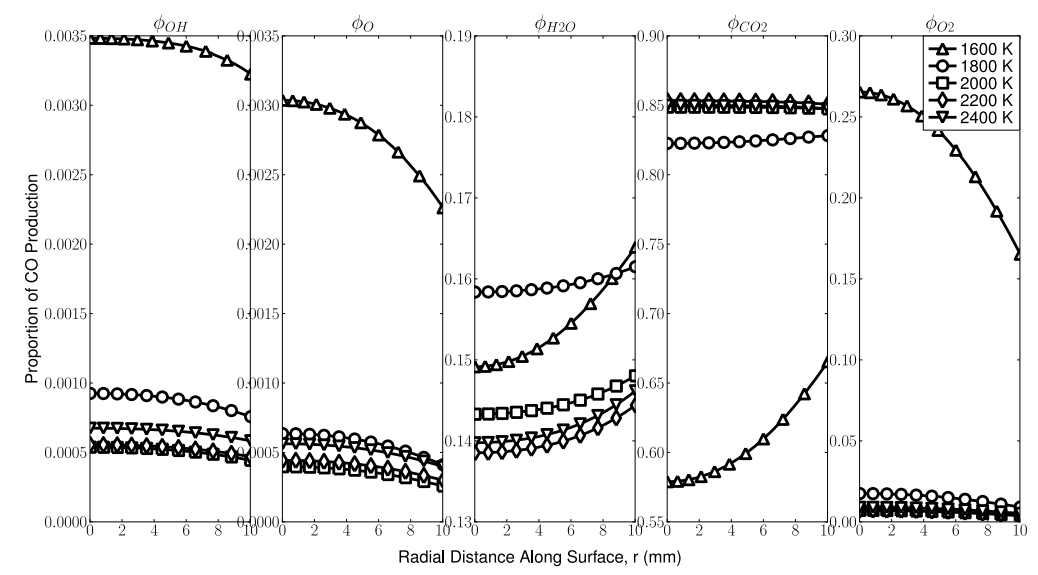

(a) Porous $\phi$

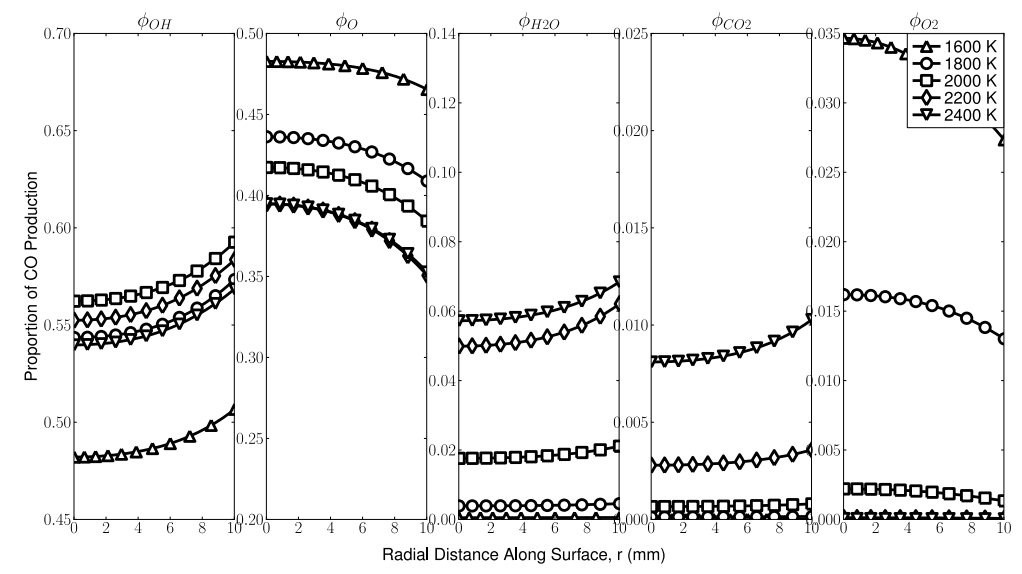

(b) Nonporous $\phi$

Figure 4.21: Homogeneous-heterogeneous coupling metric, $\phi$, for each reactant species for both porous, (a), and nonporous (b) surface reaction models.

Like the flat plate calculations, the $\mathrm{CO}_{2}+\mathrm{C}_{s}$ reaction was neglected in the nonporous 
model because it contributed less that one percent of $\mathrm{CO}$ production. Figure 4.22 shows the mass loss rate as function of radial distance for surface temperatures $T_{s}=[1600,1800$, 2000, 2200, 2400] K, the solid lines are results with the reaction included and the dashed lines, - - , are results with the reaction neglected. A similar response to the neglect of $\mathrm{CO}_{2}$ in the flat plate configuration exists for this stagnation flow configuration. At lower temperatures, the mass loss rate where $\mathrm{C}_{s}+\mathrm{CO}_{2}$ reaction was included or not yields a 40 percent error. With increase in temperature the neglected and included cases have close agreement because finite rate effects are not rate controlling. It is important to note that the surface temperature of $2600 \mathrm{~K}$ was not considered here, like in flat plate results discussed in Chapter 3. Thus, no comment can be made on whether or not there are significant differences for $T_{s}>2400 \mathrm{~K}$ for the stagnation flow configuration like that of the flat plate results.

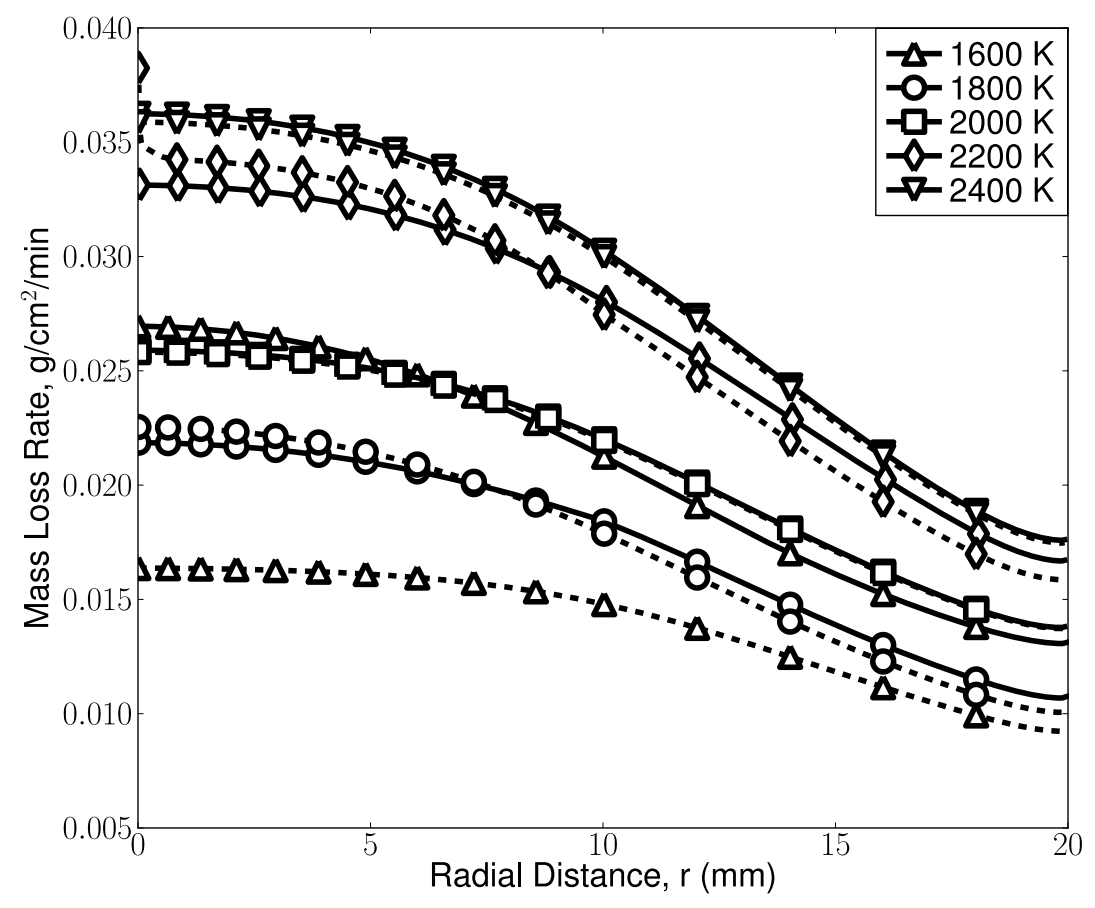

Figure 4.22: Nonporous mass loss rate with $\mathrm{C}_{s}+\mathrm{CO}_{2}$ surface reaction included (shown with solid line) and with the reaction neglected (shown with dashed, --, line) 


\section{Chapter 5}

\section{Advanced Interface Conditions and}

\section{Recommended Future Work}

This chapter presents preliminary work that considered the thermal and pressure interface conditions that were not considered in Chapters 3 and 4. Additionally, recommendations for future work to appropriately capture these boundary conditions is presented.

\subsection{The Pressure Boundary Condition}

Recall the pressure boundary condition for the reacting surface derived in Chapter 2,

$$
\frac{\partial p}{\partial n}=\vec{n} \cdot \nabla \cdot \mathbb{T}-\rho \vec{v} \cdot \nabla v_{n}
$$

The pressure gradient was updated as an external subroutine within the OpenFOAM solver. This section briefly presents the differences between using the boundary condition 5.1 and using a zero gradient pressure assumption in a stagnation flow configuration. Figures 5.1, 5.2, and 5.3 show the differences between solutions where a finite pressure gradient at the interface was calculated $\left(\frac{d p}{d n} \neq 0\right)$ and zero pressure gradient $\left(\frac{d p}{d n}\right)$ were imposed for the predicted values of pressure, $\mathrm{p}$, radial velocity, $v_{r}$, and axial velocity, $v_{z}$, respectively. The 
flow geometry considered was the same as the simplified mesh used in the stagnation flow configuration in Chapter 4. The imposed velocity boundary condition for the test of this boundary condition was plug-flow, $V_{p l u g}=1 \mathrm{~m} / \mathrm{s}$.

The differences observed on the order of less than 0.1 percent for velocity. The differences are highest near the surface for both velocity solutions. This is due to the highly viscous momentum boundary layer at the surface. The pressure differences are also small, but the location of maximum difference is approximately $1 \mathrm{~mm}$ normal distance from the stagnation plate.

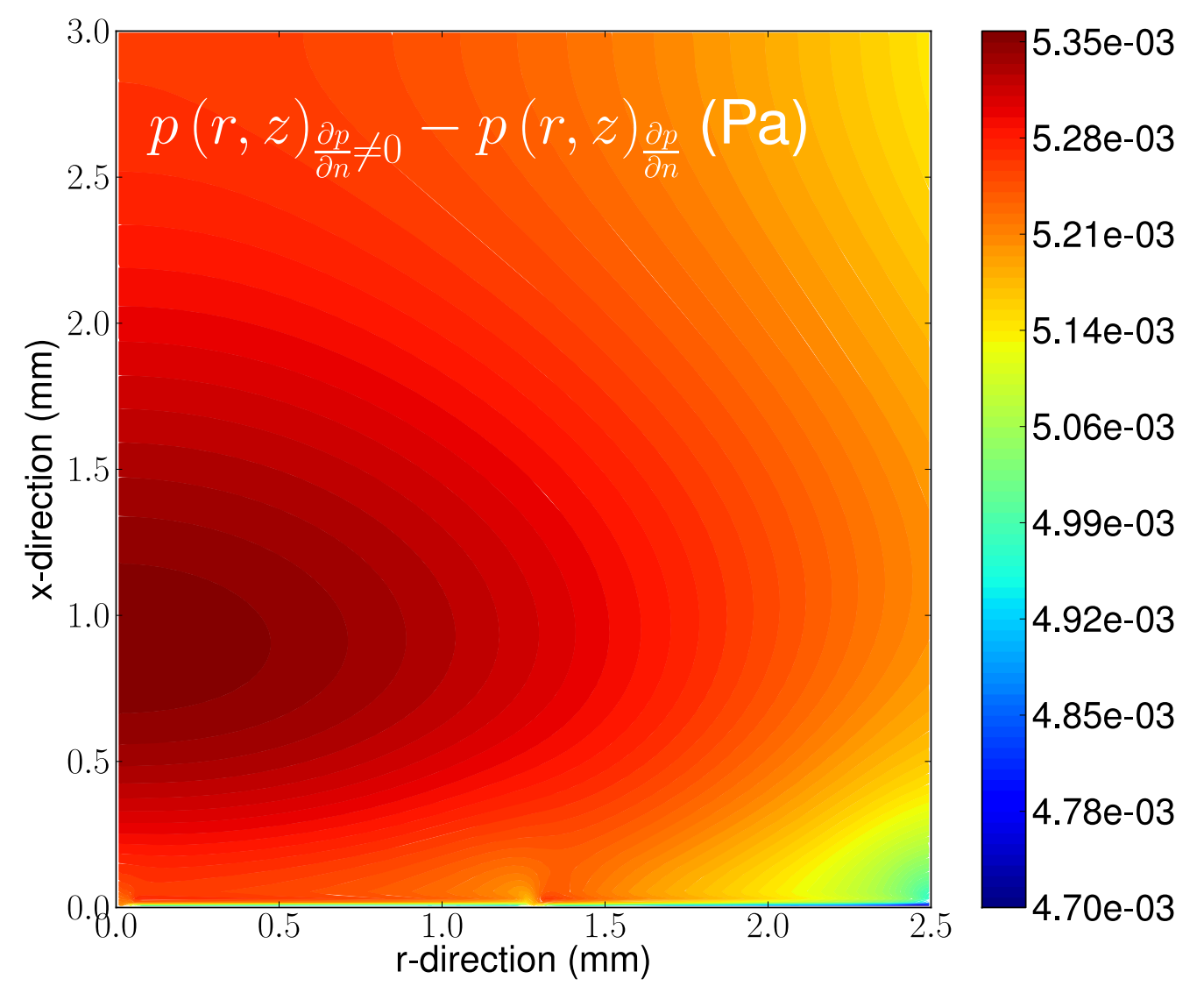

Figure 5.1: Difference in pressure fields, $p(\mathrm{~Pa})$. 


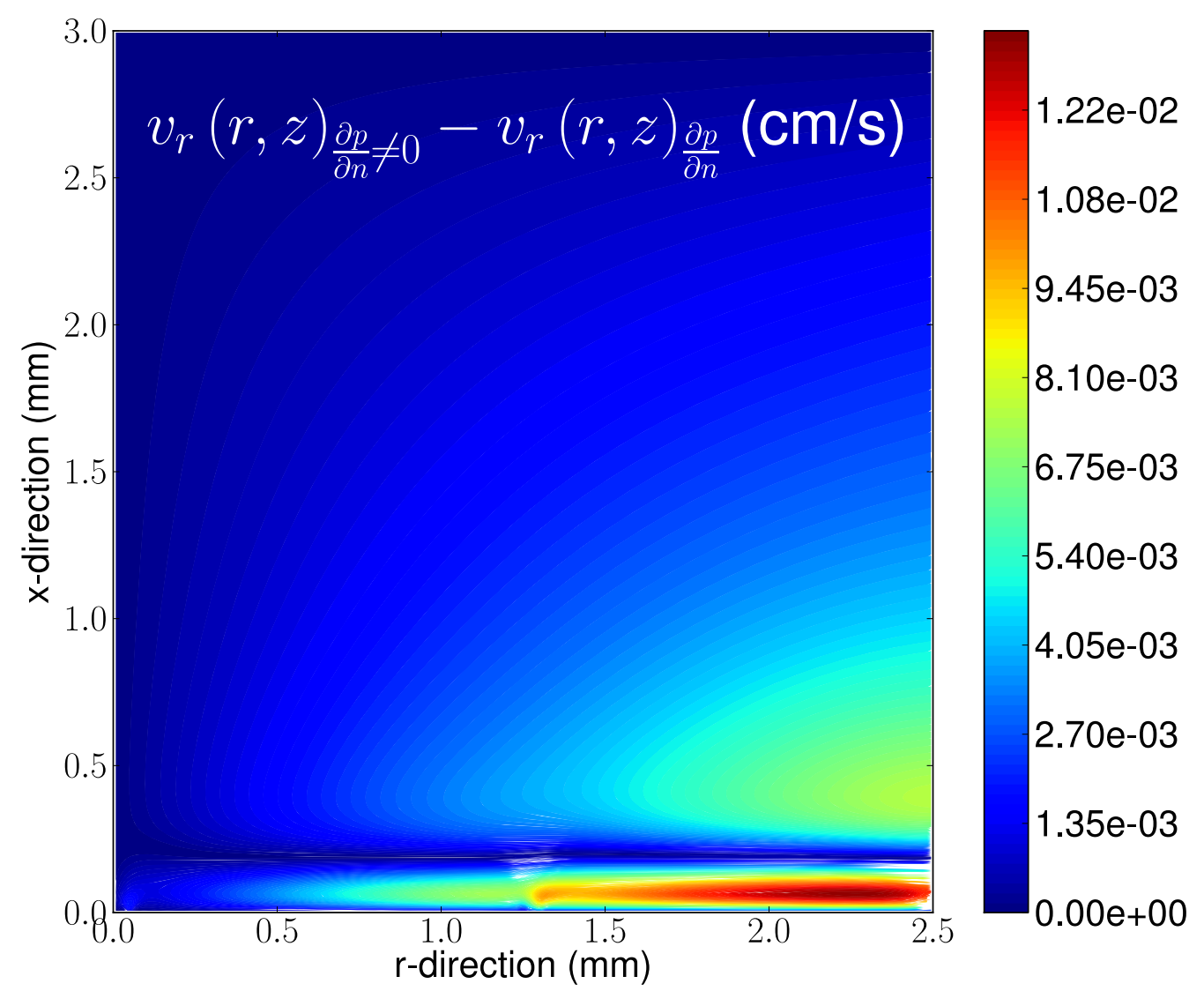

Figure 5.2: Difference in radial velocity field, $v_{r}(\mathrm{~cm} / \mathrm{s})$ 


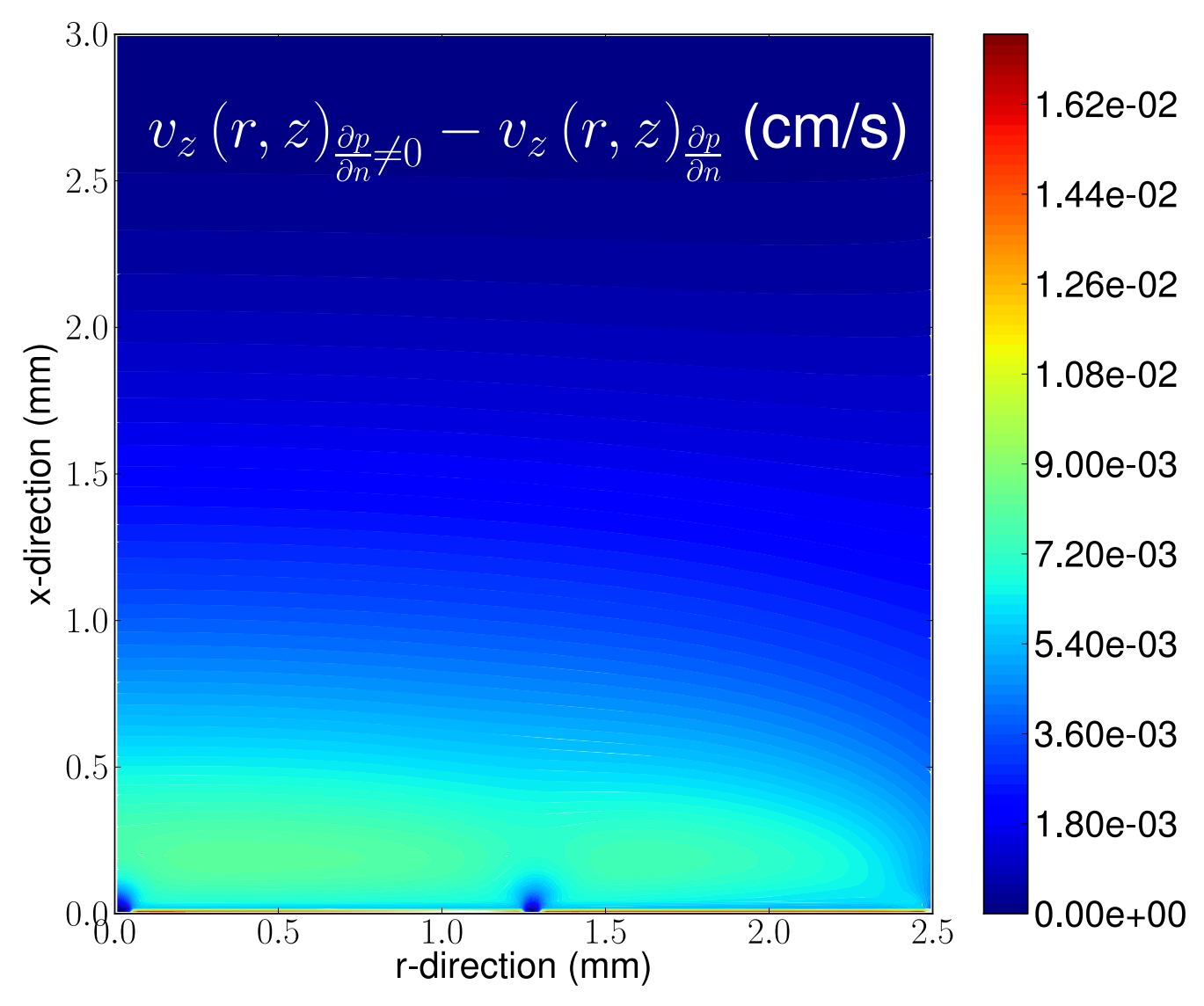

Figure 5.3: Difference in axial velocity field, $v_{z}(\mathrm{~cm} / \mathrm{s})$

Figure 5.4: Difference between stagnation flow simulations with pressure boundary condition calculated from Equation 5.1 and pressure gradient set to $\frac{\partial p}{\partial n}=0$

These results are interesting, the differences found in this elementary study are negligible. For example, changes of 0.1 percent in velocity would not affect the reacting structure. However, this study was limited due to computational resources and time. The study was not done for a flat plate, where the pressure terms are important, especially in terms of transitioning to turbulence [82]. It is therefore recommended that future work should thoroughly investigate this pressure boundary condition and resolve if it is necessary to include the viscous terms of Equation 5.1, or if it is sufficient to use the simpler to implementation, ie. widely used, zero gradient boundary condition. 


\subsection{Thermal Balance at an Interface}

In most of the parametric investigations reported in Chapters 3 and 4 , the externally controlled temperature was assumed to be constant. However, if the surface temperature is to be determined based on laser flux, then a detailed approach that includes the heat transfer into the solid carbon sample is necessary. An interface condition which included all the terms necessary to capture the heat transfer in these reacting scenarios was derived in Chapter 2. Here initial efforts that addressed these components is discussed.

Recall the thermal interface boundary condition,

$$
\frac{\partial}{\partial t}\left(\rho^{\prime} h^{s}\right)+h^{s} \rho v_{n}-\frac{\lambda_{g}}{c_{p, g}} \frac{\partial h^{s}}{\partial n}+\sum_{i=1}^{N} \rho_{g} h_{i}^{s} Y_{i} V_{i}+q_{R}-\mathcal{L}-\sum_{i=1}^{N} w_{i}^{\prime} h_{i}^{c}=h_{s} \sum_{i=1}^{N} w_{i}^{\prime}-\frac{\lambda_{s}}{c_{p, s}} \frac{\partial h_{s}^{s}}{\partial n} .
$$

All quantities, except for laser effluence $(\mathcal{L})$ and radiation $\left(q_{R}\right)$, on the left hand of Equation 5.2 side can all be readily extracted from the reacting flow simulations. The continuous laser effluence ranges from 10 to $1000 \mathrm{~W} / \mathrm{cm}^{2}$ in experiments. Through a quasi one-dimensional parameter sweep that assumed no heat conduction into the plate (consistent with Ref. [11]) and emissivity of 0.7 [83] that laser effluence values on the order of 100 $\mathrm{W} / \mathrm{cm}^{2}$ would generate surface temperatures within the ranges of Chapter 3 and 4 's results, $1600<T_{s}<2600 \mathrm{~K}$.

To test the effect of this thermal boundary condition, the thermal interface boundary condition was implemented with the laser effluence imposed as source term $\mathcal{L}$. Both constant $\mathcal{L}$ and variable $\mathcal{L}=\mathcal{L}(r)$ were considered. The variable $\mathcal{L}(r)$ used the typical laser Gaussian profile with thickness the same size of the tube diameter, $20 \mathrm{~mm}$, and $100 \mathrm{~W} / \mathrm{cm}^{2}$ average effluence. The conduction into the carbon surface was neglected and radiation was assumed to follow Stefan-Boltzmann form. Emissivity was the value of graphite carbon, $\epsilon=0.7$, from Brewster [83]. It should be noted that the author acknowledges that this radiation assumption is highly simplified and it is expanded on after the results are presented 
in this section.

Figure ?? shows the two dimensional contours for both temperature and $\mathrm{CO}_{2}$ for the stagnation flow configuration of Chapter 4 . The thermal boundary condition implemented used a Gaussian laser effluence source term, $\mathcal{L}(r)$. A parabolic velocity profile with $v_{p}=1$ $\mathrm{m} / \mathrm{s}$ was also used. The surface temperature reduces in the radial direction due to the Gaussian profile for laser effluence. This reduces the surface temperature and overall reactivity. In this non-reactive area of the surface, $\mathrm{CO}_{2}$ is produced without consumption and therefore increases in concentration. Qualitatively, these results present multidimensional features that could be orders of magnitude larger than those reported in Chapter 4.
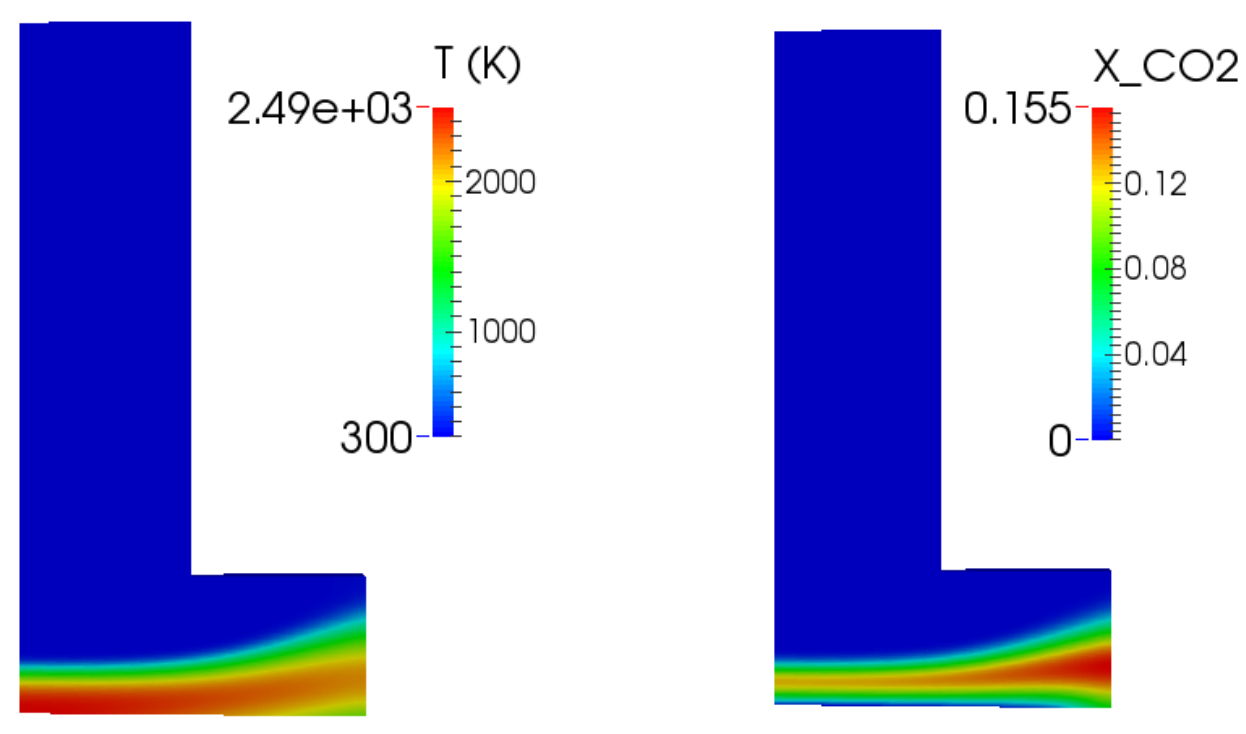

Figure 5.5: Results with Equation 5.2 used to determine surface temperature.

By normalizing each term in Equation 5.2 by $\mathcal{L}$, an idea of the relative importance and sensitivity of each term is obtained by averaging this normalization for the entire surface. The normalized values are reported in Table 5.1. The radiation term is clearly the dominant term, as it is 110 percent of the laser effluence; its value is negative because radiation represents loss of energy. Second to the radiation term is the heat source due to reactions, 
$\sum_{i=1}^{N} w_{i}^{\prime} h_{i}^{c}$. The creation of the species is exothermic and its average is 7.6 percent of laser effluence. The other terms all fall less than 3 percent of the laser effluence term.

Table 5.1: Terms of thermal interface condition for stagnation flow configuration with Gaussian laser effluence profile, $\mathcal{L}(r)$, (average effluence is $100 \mathrm{~W} / \mathrm{cm}^{2}$ ) and parabolic velocity profile with $v_{p}=1 \mathrm{~m} / \mathrm{s}$

\begin{tabular}{|l|c|}
\hline Term & Term Normalized by $\mathcal{L}$ \\
\hline$h^{s} \rho v_{n}$ & 0.016 \\
\hline$-\frac{\lambda_{g}}{c_{p, g}} \frac{\partial h^{s}}{\partial n}$ & 0.024 \\
\hline$\sum_{i=1}^{N} \rho_{g} h_{i}^{s} Y_{i} V_{i}$ & 0.011 \\
\hline$q_{R}$ & -1.08 \\
\hline$\sum_{i=1}^{N} w_{i}^{\prime} h_{i}^{c}$ & 0.076 \\
\hline$h_{s} \sum_{i=1}^{N} w_{i}^{\prime}$ & 0.011 \\
\hline
\end{tabular}

It became clear from the these initial test cases that a thorough thermal analysis of this system is needed. The investigation would be very time expensive which is why it was not pursued much further in this work. The following are recommendations for the future work that will investigate the thermal boundary condition:

1. A full conjugate heat transfer model that couples the heat transfer into the carbon surface and the reacting gas phase is necessary.

To date, Thaker and Yang [53] is the only work that this author is aware of that attempted to handle the heat transfer into the carbon surface for a multidimensional reacting flow problem. All other works generally neglect the heat transfer by assuming the gas conductivity as large $\lambda_{s} \gg \lambda_{g}$. However, if any insight is gained from 
Chapters 3 and 4, it is that such negligence is not an appropriate option until a proper test is performed for these reacting interfaces. There is a large amount of uncertainty with what the actual thermal gradient is into the carbon surface. One could construct an analytic approach to handle the heat transfer term; however, the far field boundary conditions and edge effects would need to be appropriately handled.

2. A thorough investigation of graphite radiation terms is imperative.

In this study, radiation is on the same order of the laser effluence, and exceeds the other terms in the conservation equation by an order of magnitude. By altering the emissivity from 0.5 to 0.9 , it was shown that this correspondence to laser effluence was not changed. In fact, preliminary work could achieve reductions of the normalized radiation term from 1.08 (reported case) to 0.97 .

3. An investigation of thermal interface terms is needed, especially one that takes into account more detailed surface energy considerations.

After personal correspondence with the University of Virginia's Nanoscale Lab, specifically Mr. Nam Le, the author became aware of carbon surface energies, see Ref. [84]. Energized carbon surfaces vibrate differently than carbon within a solid substrate. Therefore, it is the author's belief that using regular solid carbon enthalpy polynomial fits to calculate the surface enthalpy is insufficient.

One realization made from the thermal interface condition investigation is that a radial variation in heating does not affect the axis of symmetry mass loss rate. Figure 5.6 shows the mass loss rate for three cases with different thermal interface conditions. The first case is the previously mentioned Gaussian laser effluence source, the second is the constant laser effluence source, and the third is a fixed surface temperature, $T_{s}$, that matches the axis of symmetry surface temperature, $T_{a x}$, calculated from the first case. All cases were performed with the same parabolic velocity, $v_{p}=1 \mathrm{~m} / \mathrm{s}$. 


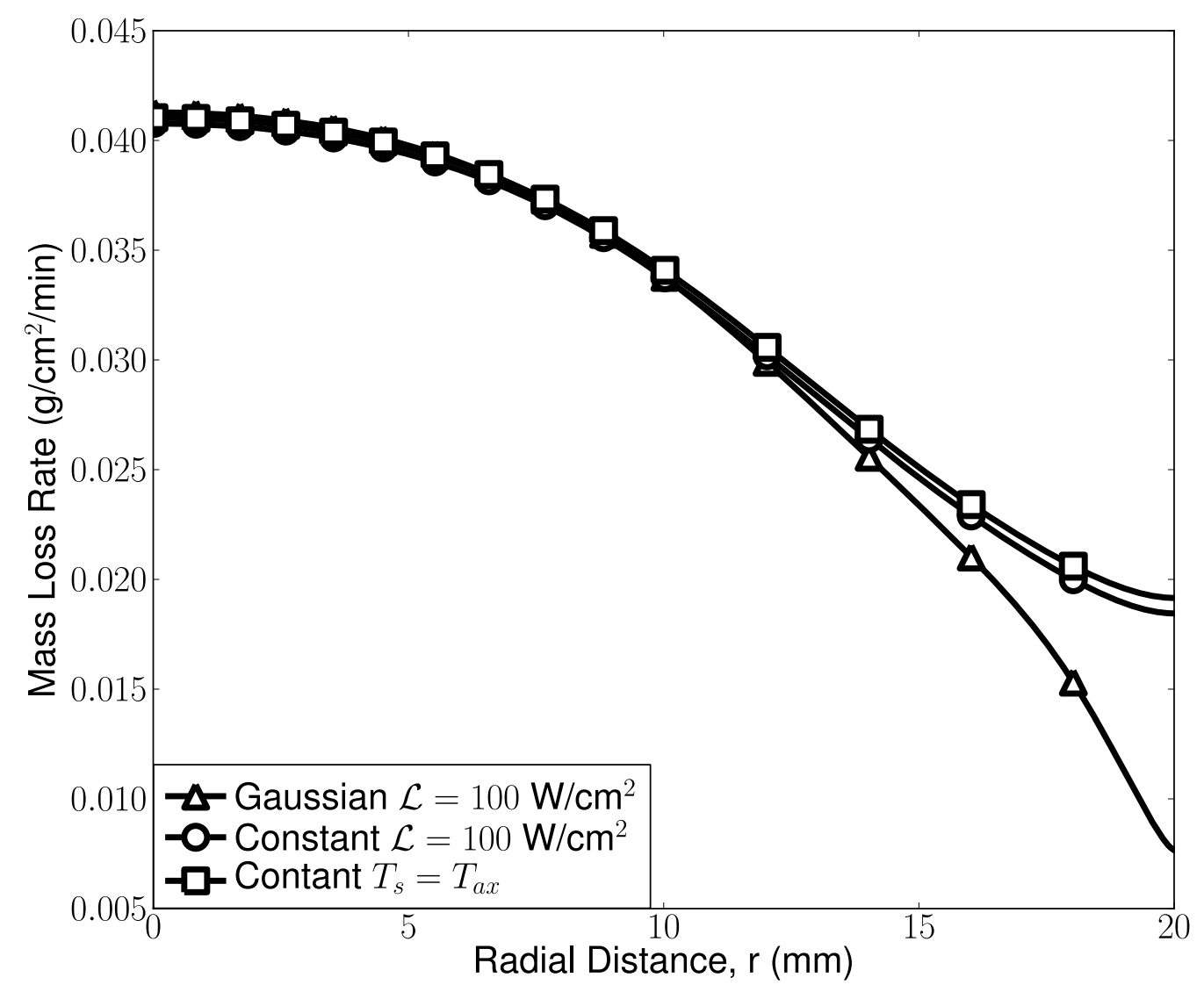

Figure 5.6: Mass loss rate for three different thermal interface conditions: (1) Gaussian $\mathcal{L}(r),(2)$ constant $\mathcal{L}$, and (3) constant surface temperature, $T_{s}$, fixed to the calculated axis of symmetry temperature from (1).

Near the axis of symmetry $(<10 \mathrm{~mm})$ the mass loss rate is the same. This means that radial effects, such as increase in $\mathrm{CO}_{2}$ concentration in the Gaussian heating case, that are not as prevalent in the constant heating cases reported in Chapter 4, do not affect the axis of symmetry mass loss rate. This also means that accurate surface temperature measurements would generate the same mass loss rate, and possibly the same solutions, as a simulation that set the thermal boundary to the experimentally determined fixed temperature profile. 


\subsection{Transferable Method and Complicated Geometries}

The Combustion and Energy Lab at the University of Virginia plans to use the solver developed in this work to better understand catalytic surface reactions with applications to using endothermic cooling in hypersonic vehicles. Chapter 2 derived the surface conservation conditions for a general case. This means that the developed solver is not limited to simulating carbon surfaces. The catalytic research will be able to use this solver to simulate the catalytic surfaces. Additionally, the realizations made in this dissertation create a framework for the possible development and validation of catalytic surface reaction models.

The solver created for this application is transferable to geometries beyond orthogonal surfaces. The simplicity of the reacting interface boundary conditions is that they are only concerned with directions normal to the surface. These normal directions are readily accessible in OpenFOAM, and the solver was developed with the idea that three-dimensional and curved/sharp reacting surfaces may one day need to be simulated.

As a proof of concept exercise, an axisymmetric reacting sphere was simulated. Inflow velocity was $10 \mathrm{~m} / \mathrm{s}$ and carbon surface temperature was a constant $2200 \mathrm{~K}$. Figure 5.7 shows the $\mathrm{CO}_{2}$ contours with white lines representing the fluid stream lines. The recirculation zone in the downstream location of the reacting sphere has the highest concentration of $\mathrm{CO}_{2}$ because of the long residence time in that location. 


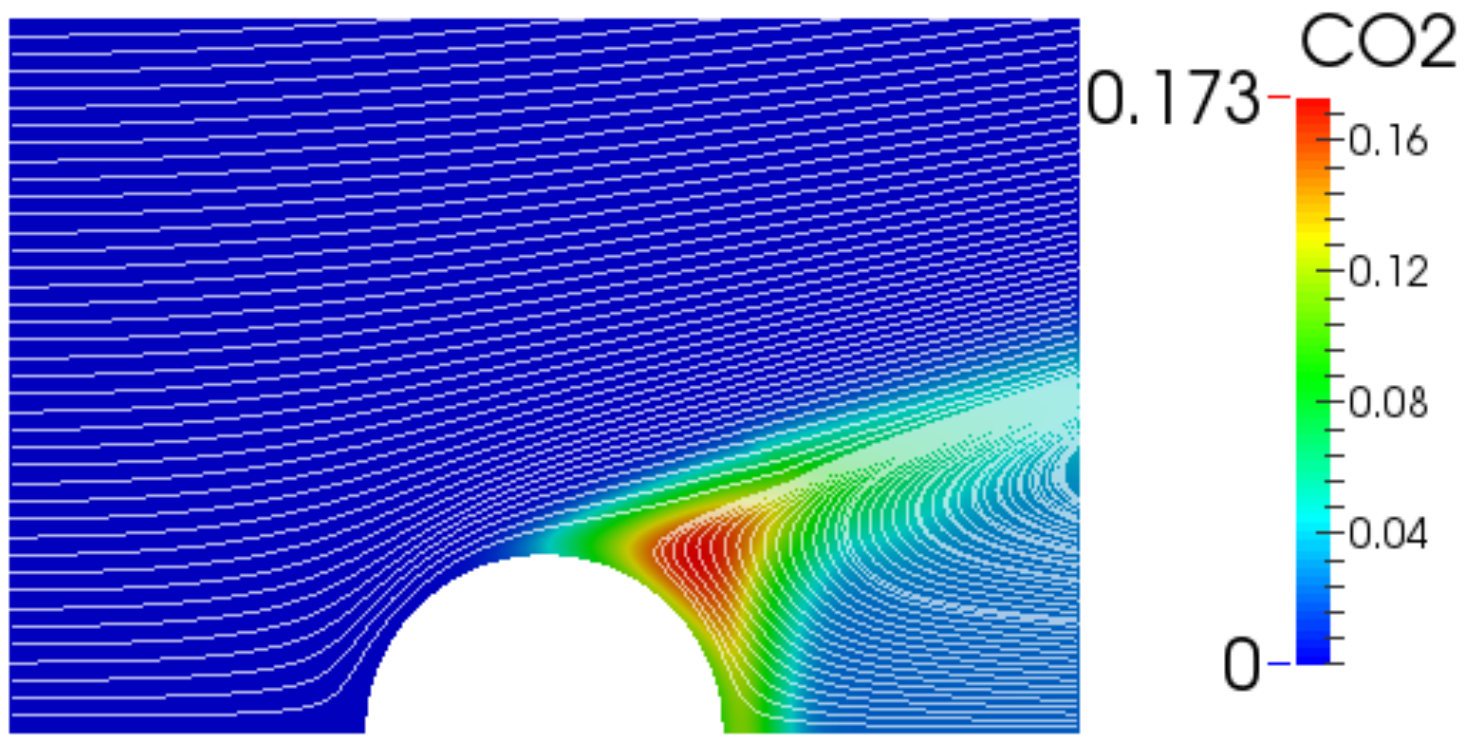

Figure 5.7: $\mathrm{CO}_{2}$ contour for a reacting carbon sphere in air heated sphere. White lines show stream lines with flow direction in the towards the right. 


\section{Chapter 6}

\section{Summary}

This work focused on investigating the multidimensional coupling of homogeneous and heterogeneous reactions in two fundamental fluid dynamic configurations: flow over a flat plate, and stagnation flow. Both fundamental carbon surface oxidation phenomena as well as assisting experiments at the Air Force Institute of Technology (AFIT) through simulations were explored. The objective of the cooperation with AFIT was to guide experimental configurations that can be constructed to investigate how a high energy laser interacts with surfaces. The project is initially using carbon surfaces of varying porosity due to the well established existing literature on carbon surface heterogeneous reaction rates. No existing framework was available to perform this investigation. This resulted in the construction of a multidimensional reacting flow solver with finite-rate chemistry and heterogeneous reacting interfaces. The solver was constructed using the OpenFOAM computational package.

The main contributions of this work are as follows;

1. The neglect of heterogeneous reactions based on a metric used to quantify homogeneousheterogeneous coupling significantly changes results.

- This conclusion is independent of the characteristic of the flow fields because neglecting $\mathrm{CO}_{2}+\mathrm{C}_{s}$ reaction caused differences in both flat plate and stagnation flow configurations. The current philosophy in the simulation of reacting carbon surfaces 
is to neglect surface reactions when species concentrations or their contributions are small in comparison to other reactants (for example, $\mathrm{O}, \mathrm{OH}$ ). This philosophy is directly contradicted by this study and it is the authors conclusion that it is important to include highly reactive species, no matter how negligible they seem. After all, the computational cost of implementing heterogeneous semi-global reactions is not high.

2. Reacting structures are multidimensional and depend on the choice of heterogeneous model, surface temperature, and flow parameters.

- The multidimensional trends of the two flow configurations differed. The flat plate solutions exhibit strong variations in $\mathrm{O}_{2}$ concentrations because it is consumed in the leading edge and not replenished in the downstream area. Additionally, $\mathrm{CO}_{2}$ creation was a function of flow velocity which was further examined by comparing the chemical source and diffusion terms of the $\mathrm{CO}_{2}$ conservation equation. The stagnation flow results did not exhibit as strong of a variation of $\mathrm{O}_{2}$ as the flat plate results along the stagnation surface. In both flow configurations, $\mathrm{CO}_{2}, \mathrm{OH}$, and $\mathrm{O}$ reaction layers depended on flow and surface temperature parameters. In the stagnation flow configuration, the attachment-detachment of $\mathrm{CO}_{2}$ flames is related to the relative magnitudes and balance of chemical source and diffusion terms of the species conservation equation. The structure of the species conservation terms are multidimensional and an analysis of these terms resulted in a solution with radial transition from attached to detached $\mathrm{CO}_{2}$ flame.

3. There is an optimal separation distance for using the computationally efficient quasi one-dimensional solver in impinging tube-jet, specifically parabolic, flow configurations.

- The L2-norm error metric was used to find the optimal separation distance, velocity, and surface temperature to use the quasi one-dimensional formulation in impinging tube-jet flow configurations. Accompanying research, attached as Appendix A, 
evaluated an error metric analysis for counterflow flames and found optimal separation distances and diameters for combustion experiments. Despite existing literature highlighting these issues, this research is the first of its kind to expose where the quasi one-dimensional formulation fails as well as make recommendations on the optimal configurations to mitigate these errors.

4. Simulations showed multidimensional trends that experiments can be verify such trends. This would serve as a form of validation for heterogeneous carbon surface reaction models in multidimensional scenarios.

-Unfortunately buoyancy and other issues caused the initial experimental flat plate investigations to be incomparable to the computations efforts. It was then recommended to move to a stagnation point flow configuration in order to better control the fluid dynamics and make use of the quasi one-dimensional formulation for parametric investigations. These parametric investigations can then be, and were, stream-lined into two-dimensional computations. These computations exposed several trends that could be seen experimentally that should set porous and nonporous surfaces apart. For example, low temperature nonporous carbon surface reactions have a 20 percent change in concentration in the radial direction, where as changes for all other cases were less than five percent. If this trend, and others revealed in this work, can be shown experimentally, then the comparison will serve as a form of validation for carbon surface reaction models.

In addition to these contributions, advanced interface conditions for pressure and enthalpy were developed and recommendations are made for future work in Chapter 5. Considerable work needs to be done with regard to the multidimensional modeling of coupled homogeneous and heterogeneous reactions. This work provides the foundation and framework to proceed and further the world's understanding of carbon surface reactions. 


\section{Appendices}


Appendix A

\section{Counterflow Paper}




\title{
On the Axisymmetric Counterflow Flame Simulations: Is there an Optimal Nozzle Diameter and Separation Distance to Apply Quasi One-Dimensional Theory?
}

\author{
R. F. Johnson, A. C. VanDine, G. L. Esposito, and H. K. Chelliah \\ Department of Mechanical and Aerospace Engineering \\ University of Virginia, Charlottesville VA 22904-4746, USA
}

\begin{abstract}
Two-dimensional axisymmetric numerical analysis of counterflow flames was employed to better understand the applicability of the quasi one-dimensional theory of Seshadri and Williams to flames produced by small diameter convergent nozzles. The computational domain considered included the convergent sections of two opposed nozzles as well as the surrounding inert annular co-flows. For computational efficiency, the fuel-oxidizer system of diluted hydrogen versus air in non-premixed flame mode, with a detailed chemical kinetic model and mixture-averaged transport property description was considered. With increase of nozzle diameter from $6.5 \mathrm{~mm}$ to larger values (with plug flow velocity profiles at the nozzle exit), the influence of the radial terms on eigenvalue and scalar variables has been compared. Error metric on nozzle diameter effects is presented with comparison to typical experimental measurement uncertainties. The analysis also showed that for nozzle separation distances below the free-floating limit, the self-similar function in quasi one-dimensional formulation can be preserved by specifying radial velocity boundary conditions, as long as the radial gradients are negligible.
\end{abstract}

Keywords: Counterflow flames; quasi one-dimensional theory; Nozzle separation distance; Nozzle diameter

\section{Introduction}

The theory that reduces the two-dimensional axisymmetric counterflow or opposed-jet flame configuration to a quasi one-dimensional (1D) formulation is well established and widely used throughout the combustion community (Seshadri \& Williams (1978); Kee, Miller, Evans \& Dixon-Lewis (1989); 
Smooke, Crump, Seshadri \& Giovangigli (1991); Kee, Coltrin \& Glarborg (2003)). The key assumptions in the derivation of quasi 1D theory are (i) description of the radial flow velocity via a self-similarity function, $v_{r}=r U(z)$, and (ii) zero radial gradients of all scalar variables along the axis of symmetry. A direct consequence of approximations (i) and (ii) is that the radial pressure-gradient, $\Lambda=(1 / r)(\partial p / \partial r)$, is treated as a constant eigenvalue along the axis of symmetry. The validity of this theory has been well established by Seshadri and co-workers (Puri \& Seshadri (1986)) in experiments using large diameter nozzles with screens (typically of the order of $25 \mathrm{~mm}$ or greater in diameter) to produce a uniform axial velocity profile at the nozzle exits (see Fig. 1a), with a nozzle separation distance of about $14 \mathrm{~mm}$.

The above ideal large diameter nozzle counterflow configuration cannot always be realized in experiments. For example, in conducting studies at extreme pressure or strain rate conditions, two-phase flows, or when performing advanced laser based diagnostics, small diameter nozzles are beneficial and have been explored by many researchers (Wu \& Law (1984);Mittal, Pitsch \& Egolfopoulos (2012)). The inconsistencies that could arise from applying quasi 1D theory of Seshadri \& Williams (1978) to small diameter nozzles has been a topic of great interest. Specifically, it is important to quantify the uncertainties introduced in applying quasi 1D theory to counterflow flames if such data is to be used in chemical kinetic model optimization or validation investigations.

As first shown by Rolon, Veynante, Martin \& Durst (1991), experiments with converging nozzles with large area ratios (without screens) suffer from non-uniform axial velocities as the nozzle separation distance is reduced from an ideal separation distance that yielded a plug flow profile (see Fig. 1b). A consequence of such non-ideal flow separation distance is that the assumed zero axial velocity gradient in the axial direction at the nozzle exit, $\partial v_{z} / \partial z=0$ (or $U(z)=0$ via continuity equation $\partial\left(\rho v_{z}\right) / \partial z=-2 \rho U(z)$ with constant density in the cold invicid flow region), is not satisfied in such experiments. A method for correcting this inconsistent finite $U(z)$ in quasi 1D simulations with non-ideal nozzle separation distances was first proposed by Chelliah, Law, Ueda, Smooke \& Williams (1991). Subsequent work by Dimotakis and co-workers (Bergthorson, Sone, Mattner, Dimotakis, Goodwin \& Meiron (2005));Bergthorson, Salusbury \& Dimotakis (2011)) and by Sarnacki, Esposito, Krauss \& Chelliah (2012) further highlighted the importance of proper boundary conditions with nozzle separation distance. In particular, for premixed flames stabilized over a stagnation plate with heat 
losses, Bergthorson et al. (2005); Bergthorson et al. (2011) have shown that the reacting viscous boundary layer is invariant with the nozzle separation distance once the nozzle separation distance is in the free-jet regime. It is important to note that the quasi 1D theory cannot be applied to freejet regime irrespective of the nozzle diameter. In contrast to stagnation flame-plate configuration, counterflow or opposed-jet laminar flames cannot be stabilized at large nozzle separation distances without some anchoring mechanism (see Fig. 1b). The nozzle separation distance at which they are barely anchored is identified as the freely-floating regime, $L=L_{F F}$ (Pellett, Northam \& Wilson (1991); Sarnacki et al. (2012)). Moreover, Sarnacki et al. (2012) experimentally demonstrated that as this ideal nozzle separation distance that yields a plug-flow profile is approached, the flame extinction strain rate defined by the Seshadri and Williams theory (identified here as $a_{\text {theory }}$ ) agrees with the local extinction strain rate (identified as $a_{\text {local }}$ ), within the experimental uncertainties.

The first two-dimensional numerical investigation on small diameter nozzles in counterflow flames was performed by Frouzakis, Lee, Tomboulides \& Boulouchos (1998) using the axisymmetric configuration. The main focus of their investigation was the effect of nozzle exit velocity profiles (i.e. plug vs. parabolic flow) on predicted non-premixed flame structure, for a specific nozzle diameter $(D=10 \mathrm{~mm})$ and separation distance $(L=10 \mathrm{~mm})$. In their axisymmetric simulations, the pressure eigenvalue, $\Lambda$, was shown to deviate from the constant value in quasi 1D formulation, especially in the cold region where local flow strain rate is quantified. It was reported that this deviation reduced as the diameter of the nozzle was doubled, without providing any quantitative information. The study's main conclusion was that quasi 1D formulation is adequate as long as a uniform velocity or plug flow condition is satisfied at the nozzle exit, without any explicit recommendation or guidance on preferred $L$ or $D$ to be used in experiments. In a more recent axisymmetric numerical investigation on twin-premixed counterflow flames using $14 \mathrm{~mm}$ nozzles, Mittal et al. (2012) have shown that the inclusion of radial heat conduction term in quasi $1 \mathrm{D}$ simulations has much greater effect than the eigenvalue departure from a constant value. The main goal of the investigation was to determine the flame stretch effects on premixed burning velocity. Bouvet, Davidenko, Chauveau, Pillier \& Yoon (2013) also performed axisymmetric numerical investigations on premixed flame-plate configuration using a $7 \mathrm{~mm}$ nozzle, including experimental measurement of the velocity field. By considering a non-reacting case with an equivalent 
$L>L_{F F}$ (i.e. a separation distance greater than the free-floating regime where quasi 1D theory does not apply), they came to the conclusion that quasi 1D theory fails to represent the stagnation flow-plate configuration. In the reacting case, with an effective nozzle separation distance less than the free-floating regime $\left(L<L_{F F}\right)$ due to thermal expansion of the reacting mixing layer, the subtle differences of the axial velocity predicted using axisymmetric and quasi 1D formulations also highlighted the inadequacy of the quasi 1D theory.

None of the aforementioned studies systematically addressed the effects of nozzle diameter on the neglected radial gradients in quasi 1D formulation. As mentioned earlier, Frouzakis et al. (1998) suggested that doubling the nozzle diameter could reduce the eigenvalue non-uniformity in the cold region. Furthermore, they stated that the average value of the eigenvalue in axisymmetric simulation is close to the constant eigenvalue assumed in quasi 1D simulations, hence the quasi 1D formulation is acceptable for the small diameter nozzle considered. In experiments, Sarnacki et al. (2012) attempted to address the nozzle diameter effect on counterflow flame extinction limits, however, the two nozzles used $(7 \mathrm{~mm}$ and $15 \mathrm{~mm})$ were not identical in area contraction ratio leading to two different boundary layers at the nozzle exits and they failed to draw any meaningful conclusions. Here, by using a consistent nozzle geometry generated by minimization of Görtler vorticity formation (see Bergthorson et al. (2005)), the effects of nozzle diameter vs separation distance on the quasi 1D formulation is analyzed numerically.

In the axisymmetric numerical analysis presented here, the OpenFOAM computational package was used to integrate the governing equations with a Finite Volume Method solver, that includes the detailed transport and chemical kinetic models (Jasak, Jemcov \& Tukovic (2007)). To lower computational cost, diluted hydrogen vs air counterflow non-premixed flame with a modest extinction strain rate of around $400 \mathrm{~s}^{-1}$ was considered. Such strain rates are close to the extinction limits of methane-air nonpremixed flames and other mildly diluted hydrocarbon-air extinction limits, hence these conditions are of great relevance. In addition, in both axisymmetric and quasi 1D simulations, the Soret effects were neglected. Several computational geometries corresponding to a Seshadri type and small diameter nozzle type configurations (see Fig. 1) were considered to highlight the differences between quasi 1D and full counterflow geometry simulations on flame structure as well as extinction limit predictions. 


\section{Governing Equations and Numerical Ap- proach}

The reacting Navier-Stokes equations that describe the conservation of mass, momentum, energy and species of axisymmetric counterflow field are well known (Williams (1985); Kee et al. (2003)), and for brevity, only the essential features are presented here. A key difference between the 2D axisymmetric (OpenFOAM) and the quasi 1D implementation is that the equations are written in conservative and non-conservative form, respectively (see Appendix A for a complete mathematical formulation). The two conservation equations of importance for the present discussion are the steady state radial momentum and the energy conservation equations - in 2D axisymmetric implementation:

$$
\begin{gathered}
\rho v \frac{\partial v_{r}}{\partial z}+\rho v_{r} \frac{\partial v_{r}}{\partial r}=-\frac{\partial p}{\partial r}+\frac{\partial}{\partial z}\left(\mu \frac{\partial v_{r}}{\partial z}\right)+\underbrace{\left\{\frac{\partial}{\partial r}\left(\frac{\mu}{r} \frac{\partial v_{r}}{\partial r}\right)\right\}}_{\text {Term } 1} \\
\frac{\partial}{\partial z}\left(\rho v h_{s}\right)-\frac{\partial}{\partial z}\left(\rho \alpha \frac{\partial h_{s}}{\partial z}\right)+\frac{\partial}{\partial z}\left(\sum_{i=1}^{N} \rho h_{i}^{s} \vec{V}_{z, i}\right) \\
=-\sum_{i=1}^{N} h_{i}^{0} w_{i}+\underbrace{\left\{-\frac{\partial}{\partial r}\left(\rho v_{r} h_{s}\right)+\frac{\partial}{\partial r}\left(\rho \alpha \frac{\partial h_{s}}{\partial r}\right)-\frac{\partial}{\partial r}\left(\sum_{i=1}^{N} \rho h_{i}^{s} \vec{V}_{r, i}\right)\right.}_{\text {Term } 2})
\end{gathered}
$$

and in quasi $1 \mathrm{D}$ theory implementation with $v_{r}=r U(z)$ :

$$
\begin{gathered}
\rho v \frac{\partial U}{\partial z}+\rho U^{2}=-\frac{1}{r} \frac{\partial p}{\partial r}+\frac{\partial}{\partial z}\left(\mu \frac{\partial U}{\partial z}\right) \\
\rho v \frac{\partial h_{s}}{\partial z}-\frac{\partial}{\partial z}\left(\alpha \frac{\partial h_{s}}{\partial z}\right)+\frac{\partial}{\partial z}\left(\sum_{i=1}^{N} \rho h_{i}^{s} V_{z, i}\right)=-\sum_{i=1}^{N} h_{i}^{0} w_{i} .
\end{gathered}
$$

In Eqs. 1 and 2, the Term1 and Term2 are the contributions due to radial effects, for example if the flame should experience any radial gradients due to small nozzle diameters.

In the OpenFOAM solver, the 2D axisymmetric conservation equations (Eqs. A.1-A.4, 2) were integrated using the Finite Volume Method in a 
segregated manner. The finite volume terms were calculated using secondorder accurate Total Variation Diminishing (TVD) Van-Leer schemes (Hirsch (2007)). For low-speed reacting flows, an iterative process was implemented to solve for the pressure field as there is no efficient explicit equation for pressure at low Mach numbers. This solver used the the Pressure Implicit Splitting of Operators (PISO) method (Issa (1986)) which iterates between the solved velocity field and a guessed pressure field until a specified numerical tolerance is met. For the hydrogen-air system considered, the chemical source terms were described using a detailed chemical kinetic model consisting of 11 species in 29 reactions, which was extracted from the JetSurf2.0 model (Wang, Dames, Sirjean, Sheen, Tangko, Violi, Lai, Egolfopoulos, Davidson, Hanson, Bowman, Law, Tsang, Cernansky, Miller \& Lindstedt (2011)). All transport and thermodynamic quantities were calculated using the same approach as the Sandia Transport Package (Kee, Miller \& Warnatz (1986)) and thermodynamic database (Kee, Rupley \& Miller (1993)), respectively, and were integrated into the OpemFOAM solver. To verify the implementation of the OpenFOAM solver, several canonical reacting flow configurations were considered and are described in the Appendix.

In the quasi 1D implementation, the conservation equations were integrated using Smooke's counterflow code (Smooke et al. (1991)) as well as the Sandia Oppdif code (Lutz, Kee, Grcar \& Rupley (1997)). The same chemical kinetic model parameters as well as the transport coefficients were implemented for comparisons with the 2D axisymmetric (OpenFOAM) solution.

\subsection{Computational Domain}

For a Seshadri type burner having large diameter nozzles $(D=26 \mathrm{~mm})$, an axisymmetric computational domain having a wedge shaped mesh (see Fig. 2a) with uniform velocity profiles at fuel and air exit planes was considered (i.e. without nitrogen co-flow), as shown in Fig. 2b. For smaller diameter nozzles, a computational domain with similar wedge shape mesh but with full details of the converging nozzles with annular inert nitrogen co-flow was considered, as shown in Fig. 2c. As mentioned earlier, the fuel and air nozzle shapes considered were similar to those used in experiments by Sarnacki et al. (2012) which were designed to minimize vorticity generation in the convergent section. In order to analyze the small diameter effects, two grids were generated with inner nozzle diameters of 6.5 and $13 \mathrm{~mm}$. Together 
with Seshadri type configuration described above, the nozzle diameter effects were analyzed for $D=6.5,13$, and $26 \mathrm{~mm}$. To address the nozzle separation distance effects, several computational grids were generated for $D=6.5 \mathrm{~mm}$ nozzles $(L=4$ and $8 \mathrm{~mm})$ and for $D=13 \mathrm{~mm}$ nozzles $(L=5,9,13,16$ and 19.5 $\mathrm{mm})$.

Various structured grid refinement strategies were explored depending on the complexity of the computational domain. For example, for the Seshadri type burner computational domain (Fig. 2b), a uniform grid spacing of 15 $\mu \mathrm{m}$ was used for all the cases with excellent agreement with the quasi 1D solutions. For more complex small nozzle diameter computational domains, both uniform and stretched grids were considered. In the unstretched regions, a uniform grid spacing of $30 \mu \mathrm{m}$ was maintained with a maximum aspect ratio of 2.0. To establish grid independence, in the reacting layer the grid was refined for several cases to achieve a $15 \mu \mathrm{m}$ grid spacing and the steadystate solutions confirmed that the results were in the asymptotic range with only $3 \mathrm{~K}$ change in temperature between 30 and $15 \mu \mathrm{m}$ solutions.

\subsection{Boundary Conditions}

Figure $2 \mathrm{~b}$ shows the computational domain simulated for the Seshadri type burner, while Fig. 2c shows the domain for a small diameter nozzle that included the entirety of the fuel, air, and co-flow nozzles, mixing region, and outflow region. In the $2 \mathrm{D}$ axisymmetric simulations, the inflow planes were different depending on the computational geometry considered. The boundary conditions imposed at the inflow planes consisted of fixed velocity plug flow, Neumann condition for pressure, fixed ambient temperature $\left(T_{0}=300 \mathrm{~K}\right)$, and fixed species mole fractions (diluted hydrogen $\left(X_{\mathrm{H}_{2}}=0.16\right)$ vs. air $\left.\left(X_{\mathrm{O}_{2}}=0.21\right)\right)$. The inclusion of the full nozzle geometry in the small diameter simulations allowed the plug flow upstream velocity to develop into the expected nonuniform velocity profiles at the nozzle exits. The plug-flow velocities prescribed were varied to explore strain effects on the flame. However, the precise local strain rate specification was difficult due to variation in nozzle exit velocity profile depending on the nozzle separation distance and thermal expansion. In the small diameter nozzle case, the inert nitrogen co-flow momentum was always matched with the momentum of the inner jet. This assured that the shear layer formed between the inner and the outer streams did not influence the flow divergence. Outflow boundary conditions of the computational domain were similar for all cases and were prescribed 
by fixing far-field pressure nodes and Neumann conditions for all other variables. Nozzle walls and edges were described by no-slip, viscous boundary conditions by setting normal and tangental velocity components to zero, and all other variables were described by Neumann conditions at the wall face. Chemical reactions were initiated by imposing a Gaussian temperature profile over a non-reacting diffusion solution and residuals were monitored to assure convergence for all solutions.

In the quasi 1D flame solutions, the pressure was held constant at the average value determined from axisymmetric simulations and the temperature and species boundary conditions imposed were the same as those described above in the 2D axisymmetric simulations. In addition, the velocity boundary conditions $\left(v_{z}\right.$ and $\left.U\right)$ in the quasi 1D formulation were matched with the OpenFOAM solutions (identified as OF) as described below: for large diameter nozzle (Seshadri type burner),

$$
z= \pm \frac{L}{2}, v_{z}=\left(v_{z}\right)_{O F}, U=0
$$

and for small diameter nozzles,

$$
\begin{aligned}
& L=L_{F F}: z= \pm \frac{L}{2}, v_{z}=\left(v_{z}\right)_{O F}, U=0 \\
& L<L_{F F}: z= \pm \frac{L}{2}, v_{z}=\left(v_{z}\right)_{O F}, U=U_{O F} \neq 0, \\
& L>L_{F F}: \text { quasi one }- \text { dimensional theory cannot be applied. }
\end{aligned}
$$

\section{Results}

\subsection{Large diameter Seshadri type burner simulations}

Based on the non-premixed flames established between opposed large diameter nozzles $(D=26 \mathrm{~mm}$ ) with plug-flow velocity profiles, the key terms that support the validity of the quasi 1D theory of Seshadri \& Williams (1978) are presented first. Figures 3 and 4 show a comparison of the velocity field (i.e. axial velocity, $v \equiv v_{z}$, and self-similarity function, $\left.U \equiv v_{r} / r\right)$ and the temperature as a function of the distance along the axis of symmetry predicted using 2D axisymmetric simulations (solid lines) and quasi 1D simulations (dashed lines). For the diluted hydrogen and air flame with a moderately high local strain rate of $a=375 \mathrm{~s}^{-1}$ (note: the local extinction strain rate 
is about $480 \mathrm{~s}^{-1}$ ), the velocity and temperature agreements are well within the numerical uncertainties (see section A.4 in the Appendix). Certainly, the differences shown between two implementations are much smaller than the current experimental measurement techniques of flow strain rates and temperature (Sarnacki et al. (2012); Figura \& Gomez (2012)).

A key observation from the above results are that (a) the self-similarity function, $U(z)$, agrees well in both cold hydrodynamic region as well as inside the reacting mixing layer region and (b) the excellent agreement implies that the radial terms included in the axisymmetric simulation are negligible. One can further evaluate the contribution of the radial terms in 2D axisymmetric formulation in comparison to the dominant axial terms. For example in the radial momentum equation, at steady state, the only term that is neglected in the quasi 1D formulation is the Term1 identified in Eq. (1). Any finite contribution of the Term1 can be thought of as the residue in Eq. (4) and will manifest as a variation of $\Lambda$ in axisymmetric simulations. This residue is identified here as $R_{\Lambda}$. Figure 5 shows a comparison of the eigenvalue, $\Lambda$, calculated from quasi $1 \mathrm{D}$ and $2 \mathrm{D}$ axisymmetric simulations, together with all other remaining terms contributing to the radial momentum equation, Eq. (1). For the present large diameter nozzle simulations, the departure of the eigenvalue from a constant value determined from the quasi 1D is very small $\left(\Lambda=-(7.6 \pm 0.2) \times 10^{-4} \mathrm{~Pa} / \mathrm{m}^{2}\right)$, especially in the cold hydrodynamic region where the flow strain rate is typically calculated. In contrast, previous work with small diameter nozzles have reported comparatively larger departure of the eigenvalue from quasi 1D theory (Frouzakis et al. (1998); Mittal et al. (2012); Bouvet et al. (2013)).

Any radial variations in counterflow flames can also influence the solution of energy and species conservation equations, i.e. scalar properties $T$ and $Y_{i}$. In Eq. (2), Term 2 identifies the radial gradient dependencies in energy conservation equation that give rise to differences between the axisymmetric and the quasi $1 \mathrm{D}$ solutions. For the present discussion, this residual term is identified as $R_{h}$. Figure 6 shows a comparison of all the terms in Eq. (2) evaluated using 2D axisymmetric and quasi 1D solutions, including the residue term $R_{h}$. A very small value of $R_{h}$ implies that for the present large diameter Seshadri type burner with plug-flow velocity profiles, the radial term in energy equation is negligible and the quasi one-dimensional theory accurately represents the $2 \mathrm{D}$ axisymmetric flow field. 


\subsection{Nozzle diameter effects}

While the concept of minimizing radial effects by increasing the nozzle diameter is not new, the main purpose of the present paper is to develop an error metric to quantify the effect of radial contributions in small diameter counterflow flames, as a function of both the nozzle diameter and the nozzle separation distance. To our knowledge, such a systematic investigation has not been reported in the literature, which is now possible due to advanced computational capabilities available. For two nozzle diameters of $D=13$ and $6.5 \mathrm{~mm}$ with a nozzle separation distance of $L=4 \mathrm{~mm}$, Figs. 7, 8, 9, 10 show comparisons of the velocity field and temperature, terms contributing to the radial momentum equation and energy conservation equation, respectively, predicted using the 2D axisymmetric simulations and quasi 1D simulations. Since $L<L_{F F}$ with finite $U$ values at the nozzle exit planes, in quasi 1D simulations, both $v_{z, \pm L / 2}$ and $U_{ \pm L / 2}$ values from $2 \mathrm{D}$ simulations are imposed. Alternatively, if these boundary conditions are available from experiments, for example from PIV measurements (Sarnacki et al. (2012)), they can be imposed in quasi 1D simulations. While the local strain rates are different for the two nozzle diameters considered $\left(a=268 \mathrm{~s}^{-1}\right.$ for $D=13 \mathrm{~mm}$ and $a=354$ $\mathrm{s}^{-1}$ for $D=6.5 \mathrm{~mm}$ ), a small but noticeable difference of the flame structure between the 2D axisymmetric and quasi $1 \mathrm{D}$ simulations is observed. In particular, the residual term $R_{h}$ in energy equation (see Fig. 11) shows an increased departure as the nozzle diameter is reduced from $13 \mathrm{~mm}$ to $6.5 \mathrm{~mm}$. A quantitative comparison of diameter effects on $\Lambda, R_{h}$, and $U$ is presented in section 3.4.

For the smaller diameter nozzles $(D=6.5 \mathrm{~mm})$, the temperature comparisons shown in Fig. 8 indicate $46 \mathrm{~K}$ difference in peak flame temperature between the 2D axisymmetric and quasi 1D simulations. This difference is certainly greater than any numerical effects discussed in the Appendix A.3 (of the order of $10 \mathrm{~K}$ ). In contrast, for the larger diameter nozzle $(D=13 \mathrm{~mm})$, difference in predicted peak temperature is $20 \mathrm{~K}$. Since accurate estimation of flame temperature is critical for chemical kinetic model validation, it is important to understand the origin of these temperature variations. The first clue is that for the $D=6.5 \mathrm{~mm}$ nozzle, the deviation of self-similar function $U$ between the two simulations is significant irrespective of the specification of the exact axial velocity and $U$ values at the boundaries (see Fig. 7). Examination of the terms contributing to the radial momentum equation (Fig. 9 ) shows that while most terms are of the same order, the eigenvalue spread 
is significantly large for the $D=6.5 \mathrm{~mm}$ case in comparison to the $D=13 \mathrm{~mm}$ nozzles. Secondly, consistent with the analysis by Mittal et al. (2012), the residue of the energy equation is dominated by the radial heat conduction term and increases with decreasing the nozzle diameter (see Fig. 11).

\subsection{Nozzle separation distance effects}

Unlike in the Seshadri type burner with screens, in small diameter nozzle experiments it is impossible to determine a priori the ideal nozzle separation distance where $U=0$ at the boundaries. This is because of the variability of the mixing layer thickness, $L_{\text {mixing }}$, which depends on the thermal expansion associated with the overall heat release and the imposed flow strain rates (or the Reynolds number). Thus, in quasi 1D simulation of small diameter counterflow experiments with an arbitrarily selected nozzle separation distance, the finite $U$ values at the boundaries must be either measured from experiments, or calculated as in present 2D simulations. The fundamental assumption in such quasi $1 \mathrm{D}$ calculations with imposed finite $U$ values is that the radial gradients are negligible irrespective of the nozzle diameter. To explore the applicability of this assumption, 2D axisymmetric calculations were carried out for several different nozzle separation distances using both nozzle diameters considered above. For brevity, only the smaller diameter nozzles results are presented here. Figures 12, 13, 14, 15 show a comparison between the 2D axisymmetric results and the quasi $1 \mathrm{D}$ simulations for $L=4$ and $8 \mathrm{~mm}$. Qualitatively, smaller separation distance simulations with imposed finite $U$ values seems to yield results in closer agreement with the $2 \mathrm{D}$ axisymmetric simulations.

\subsection{Metric for quantification of radial effects}

Instead of making qualitative comparisons between 2D axisymmetric and quasi 1D simulations, a quantitative estimate of the neglected radial terms can be evaluated by suitably defined error metric, for example the $L_{2}$-norm given by,

$$
L_{2}\left(\phi_{i}\right)=\sqrt{\frac{\sum_{j=1}^{N_{c}}\left(\phi_{i}\right)_{j}^{2}}{N_{c}}},
$$

where $\phi_{i}^{2}=\left(\Lambda_{2 D}-\Lambda_{1 D}\right)^{2}, R_{h}^{2}$, or $\left(U_{2 D}-U_{1 D}\right)^{2}$ are summed over computational cells, $N_{c}$, from $j=1, \ldots, N_{c}$ along the axis of symmetry. Figures 16 and 17 
show comparisons of such a metric as a function of the nozzle diameter and nozzle separation distance, respectively.

The large diameter Seshadri type burners clearly yield the lowest $L_{2}$ norm as seen from Fig. 16. However, it may be useful to correlate the error metric to typical measurement uncertainties and explore the existence of an optimal nozzle diameter and separation distance or a range in applying quasi $1 \mathrm{D}$ theory. To address this question, the eigenvalue variance shown in Fig. 9 is first correlated with the local strain rate variations. For example, in the case of diluted hydrogen and air flame considered, for $D=13 \mathrm{~mm}$ case, the predicted eigenvalue variation between $-2.86 \times 10^{-4}$ to $-3.4 \times 10^{-4} \mathrm{~Pa} / \mathrm{m}^{2}$ corresponds to a local strain rate variation of 267 to $275 \mathrm{~s}^{-1}$ and to a peak flame temperature variation of 1228 to $1240 \mathrm{~K}$. These variations are well within the current measurement uncertainties (Sarnacki et al. (2012); Figura \& Gomez (2012)). Even for the smaller diameter nozzle considered, the eigenvalue variation between $-5.75 \times 10^{-4}$ to $-7.0 \times 10^{-4} \mathrm{~Pa} / \mathrm{m}^{2}$ yields very modest local strain rate variations between 328 to $339 \mathrm{~s}^{-1}$ and peak flame temperature variations of 1167 to $1185 \mathrm{~K}$, again well within the experimental measurement uncertainties, but greater than the numerical uncertainties.

Figure 17 shows the variation of error metrics for the large diameter nozzle as a function of nozzle separation distance. For $L<<L_{F F}$, with the inclusion of finite radial velocity gradient effects (i.e. finite $U$ - see Eq. 7 ) in the quasi 1D calculations, increase in $L$ yields a lower error. This reduction in error can be related to lower radial gradients as the nozzles are pulled apart. However, as the nozzle separation distance approaches or exceeds the free-floating limit (i.e. $L>_{F F}$ ), where quasi 1D theory is not applicable (see Eq. 7), the error metric is seen to increase.

While this investigation considered only a limited range of flame strain rate variations (slightly away from flame extinction limit), based on above discussion, it may be reasonable to conclude that as long as finite $U$ values are imposed at the boundaries, small diameter nozzle experiments with nozzle diameters of the order of $12 \mathrm{~mm}$ or greater can be used with quasi 1D simulations. Furthermore, for $13 \mathrm{~mm}$ diameter nozzles $1<L / D<L_{F F} / D$ is seen to yield lowest departure between 2D and quasi 1D solutions. Whether this finding can be generalized to any small diameter nozzles is not clear. One essential requirement in applying the quasi one-dimensional theory is that the nozzle separation distance must lie between the free-floating distance and the mixing layer thickness. Violation of the latter will lead to heat losses at the nozzles. 


\section{Conclusions}

Two-dimensional axisymmetric counterflow reacting flow simulations were performed to investigate the applicability of quasi one-dimensional theory of Seshadri and Williams to small diameter, large area-ratio converging nozzles. Analysis was also performed with large diameter nozzles with plug flow boundary conditions (Seshadri type burners) to demonstrate the negligible effects of the radial terms in conservation equations. When applied to smaller diameter converging nozzles, the influence of non-zero contributions of radial terms on radial momentum and energy conservation equations were explicitly evaluated. Based on a normalized error metric, and the diluted hydrogen vs air non-premixed flames considered, it was suggested that nozzle diameters greater than $12 \mathrm{~mm}$ will yield errors less than numerical uncertainties and certainly well below current experimental uncertainties. With regard to nozzle separation distance, analysis was performed for distances greater than the mixing layer distance and lower than the free-floating regime distance. It was shown that, as long as radial effects are small, specification of finite values for the self-similar function at the boundaries in quasi one-dimensional simulations can predict the flow strain rates and flame temperature within numerical or experimental uncertainties.

While the verification of the two-dimensional OpenFOAM numerical solver was performed by considering chemical source term integrations, premixed flame structure solutions, and non-premixed flame extinction limit predictions using simpler Sehsadri type burner configuration, further work is needed to establish the nozzle based extinction limit predictions. Once completed, the solver developed with parallel computing capabilities can provide a valuable tool for future multi-dimensional laminar flame investigations with detailed chemical kinetic models.

\section{Acknowledgements}

Harsha Chelliah gratefully acknowledges stimulating discussions with Professor Forman Williams which led to the work described in this paper. The authors also would like to acknowledge the Air-Force High-Energy Laser Joint Technology Office (HEL-JTO) for funding, under the HEL JTO MRI Program (AFOSR-BAA-2010-2). Ryan Johnson was supported by the Depart-

ment of Defense (DoD) through the National Defense Science \& Engineering 
Graduate Fellowship (NDSEG) Program and the Virginia Space Grant Consortium (VSGC) Graduate Fellowship Program.

\section{References}

Bergthorson, J. M., Salusbury, S. D. \& Dimotakis, P. E. (2011), 'Experiments and modelling of premixed laminar stagnation flame hydrodynamics', Journal of Fluid Mechanics 681, 340-369.

Bergthorson, J. M., Sone, K., Mattner, T. W., Dimotakis, P. E., Goodwin, D. G. \& Meiron, D. I. (2005), 'Impinging laminar jets at moderate reynolds numbers and separation distances', Physical Review E $\mathbf{7 2}$.

Bouvet, N., Davidenko, D., Chauveau, C., Pillier, L. \& Yoon, Y. (2013), 'On the simulation of laminar strained flames in stagnation flows: 1D and 2D approaches versus experiments', Combustion and Flame pp. 1-15.

Chelliah, H. K., Law, C. K., Ueda, T., Smooke, M. D. \& Williams, F. A. (1991), 'An experimental and theoretical investigation of flow-field, dilution and pressure effects on the extinction condition of methane/oxygen/nitrogen diffusion flames', Proc. Combust. Inst. 23, 503-511.

Cuoci, A., Frassoldati, A., Faravelli, T. \& Ranzi, E. (2013), 'A computational tool for the detailed kinetic modeling of laminar flames: Application to $\mathrm{c}_{2} \mathrm{~h}_{4} / \mathrm{ch}_{4}$ coflow flames', Combustion and Flame 160, 870-886.

Figura, L. \& Gomez, A. (2012), 'Laminar counterflow steady diffusion flames under high pressure conditions', Combustion and Flame 159(1), 142 150 .

Frouzakis, C. E., Lee, J., Tomboulides, A. G. \& Boulouchos, K. (1998), 'Two-dimensional direct numerical simulation of opposed-jet hydrogenair diffusion flame', Proc. Combust. Inst. 27, 571-577.

Hirsch, C. (2007), Numerical Computation of Internal and External Flows, second edition edn, Butterworth-Heinemann, Oxford. 
Issa, R. I. (1986), 'The computation of compressible and incompressible recirculating flows by a non-iterative implicit scheme', Journal of Computational Physics 62, 66-82.

Jasak, H., Jemcov, A. \& Tukovic, Z. (2007), 'Openfoam: A c++ library for complex physics simulations', International Workshop on Coupled Methods in Numerical Dynamics IUC.

Kee, R. J., Coltrin, M. E. \& Glarborg, P. (2003), Chemically Reacting Flow, John Wiley and Sons, Hoboken, NJ.

Kee, R. J., Grcar, J. F., Smooke, M. D., Miller, J. A. \& Meeks, E. (1998), Premix: A fortran program for modeling steady laminar one-dimensional premixed flames, Technical Report SAND 1998, Sandia Report.

Kee, R. J., Miller, J. A., Evans, G. H. \& Dixon-Lewis, G. (1989), 'A computational model of the structure and extinction of strained, opposed flow, premixed methane-air flames', Proc. Combust. Inst. 22(1), 1479 1494.

Kee, R. J., Miller, J. A. \& Warnatz, J. (1986), A fortran computer code package for the evaluation of gas-phase multicomponent transport properties, Technical Report SAND86, Sandia Report.

Kee, R. J., Rupley, F. M. \& Miller, J. A. (1993), Chemkin-ii: A fortran chemical kinetics package for the analysis of gas phase chemical kinetics, Technical Report SAND89-8009B, Sandia Report.

Lutz, A. E., Kee, R. J., Grcar, J. F. \& Rupley, F. M. (1997), Oppdif: A fortran program for computing opposed-flow diffusion flames, Technical Report SAND 1997, Sandia Report.

Lutz, A., Kee, R. J. \& Miller, J. A. (1987), Senkin: A fortran program for predicting homogeneous gas phase chemical kinetics with sensitivity analysis, Technical Report SAND87, Sandia National Laboratories Report.

Mittal, V., Pitsch, H. \& Egolfopoulos, F. (2012), 'Assessment of counterflow to measure laminar burning velocities using direct numerical simulations', Combustion Theory and Modelling 16(3), 419-433. 
Oevermann, M., Gerber, S. \& Behrendt, F. (2009), 'Euler-lagrange/dem simulation of wood gasification in a bubbling fluidized bed reactor', Particuology 4, 307-316.

Pellett, G. L., Northam, G. B. \& Wilson, L. G. (1991), 'Counterflow diffusion flames of hydrogen, and hydrogen plus methane, ethylene, propane, and silane vs. air', AIAA Aerospace Sciences Meeting, Washington, D.C. AIAA 1991-0370.

Puri, I. K. \& Seshadri, K. (1986), 'Extinction of diffusion flames burning diluted methane and diluted propane in diluted air', Combustion and Flame 65, 137-150.

Reinelt, D., Laurs, A. \& Adomeit, G. (1998), 'Ignition and Combustion of a Packed Bed in a Stagnation Point Flow Part II: Heterogeneous and Homogeneous Reactions', 2180(97), 373-379.

Rolon, J. C., Veynante, D., Martin, J. P. \& Durst, E. (1991), 'Counter jet stagnation flows', Experiments and Fluids 11, 313-324.

Roy, C. J. \& Oberkampf, W. L. (2010), Verification and Validation in Scientific Computing, Cambridge University Press, Cambridge, UK.

Sarnacki, B. G., Esposito, G., Krauss, R. H. \& Chelliah, H. K. (2012), 'Extinction limits and associated uncertainties of nonpremixed counterflow flames of methane, ethylene, propylene and n-butane in air', Combustion and Flame 159, 1026-1043.

Seshadri, K. \& Williams, F. A. (1978), 'Laminar flow between parallel plates with injection of a reactant at high reynolds number', Int. J. Heat and Mass Transfer 21, 251-253.

Smooke, M. D., Crump, J., Seshadri, K. \& Giovangigli, V. (1991), 'Comparison between experimental measurements and numerical calculations of the structure of counterflow, diluted, methane-air, premixed flames', Proc. Combust. Inst. 23(1), $463-470$.

Wang, H., Dames, E., Sirjean, B., Sheen, D. A., Tangko, R., Violi, A., Lai, J. Y. W., Egolfopoulos, F. N., Davidson, D. F., Hanson, R. K., Bowman, C. T., Law, C. K., Tsang, W., Cernansky, N. P., Miller, D. L. \& Lindstedt, R. P. (2011), A high-temperature chemical kinetic model 
of n-alkane (up to n-dodecane), cyclohexane, and methyl-, ethyl-, npropyl and n-butyl-cyclohexane oxidation at high temperatures (jetsurf 2.0), Technical report, Combustion Kinetics Laboratory, University of Southern California.

URL: http://melchior.usc.edu/JetSurF/JetSurF2.0/Index.html

Williams, F. A. (1985), Combustion Theory, Westview Press.

Wu, C. K. \& Law, C. K. (1984), 'On the determination of laminar flame speeds from stretched flames', Proc. Combust. Inst. 20, 1941-1949. 


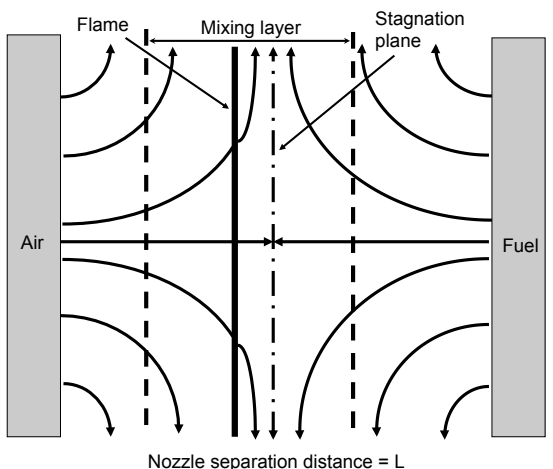

(a)

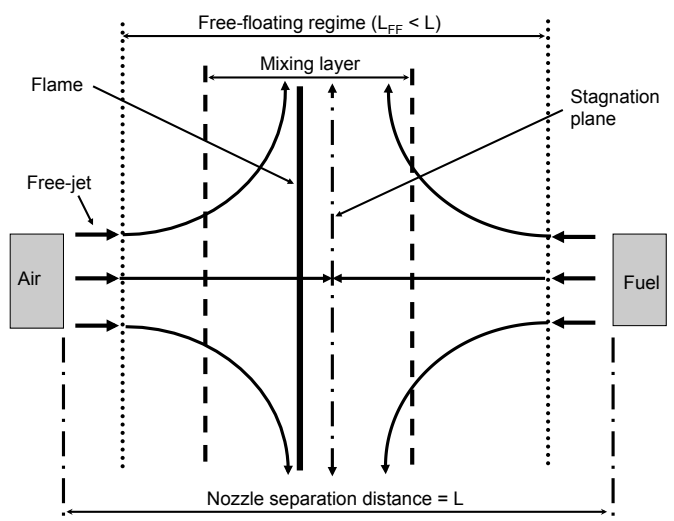

(b)

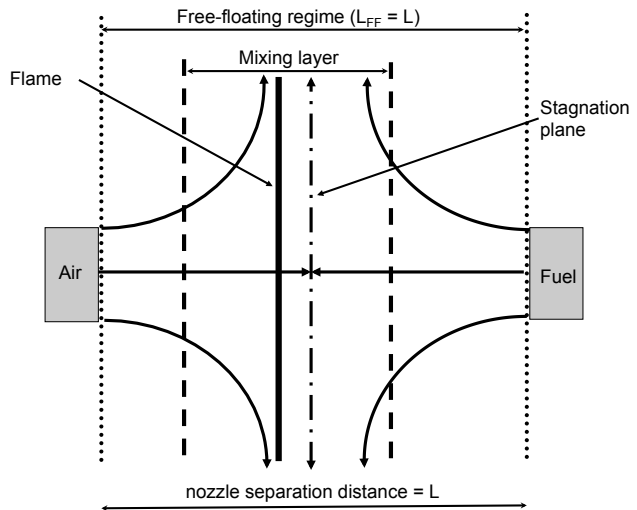

(c)

Figure 1: Schematic of counterflow diameter and separation distance effects - (a) large nozzle setup (Seshadri type burner) with plug-flow velocity profile, (b) small diameter nozzles with non-ideal separation distances ( $L>L_{F F}$ with plug flow and free-jet or $L<L_{F F}$ with finite $U$ values), and (c) small nozzles with ideal separation distanc $\left.\& \&=L_{F F}\right)$. 


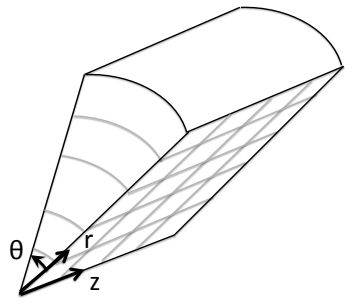

(a)

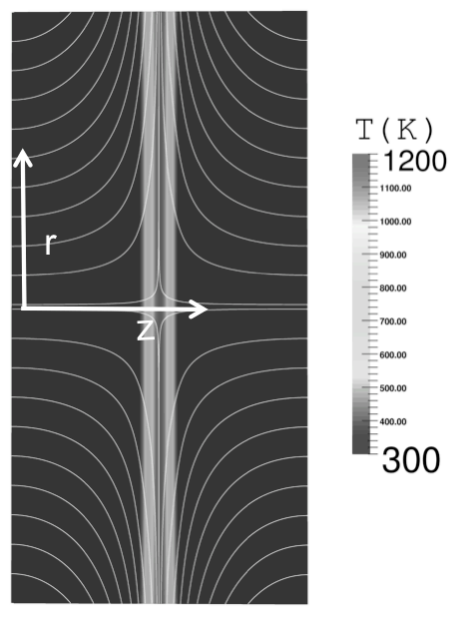

(b)

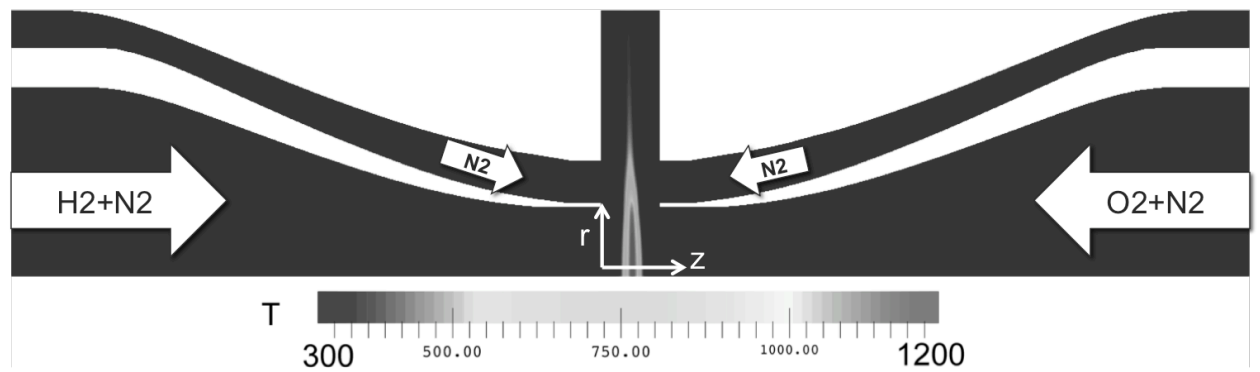

(c)

Figure 2: Computational domains implemented showing (a) 2D wedge-shape mesh with $r$ and $z$ coordinates, (b) typical solution from large diameter Seshadri type plug-flow burners, and (c) solution from a small diameter nozzle type burners. Please see the online version to view these figures in color. 


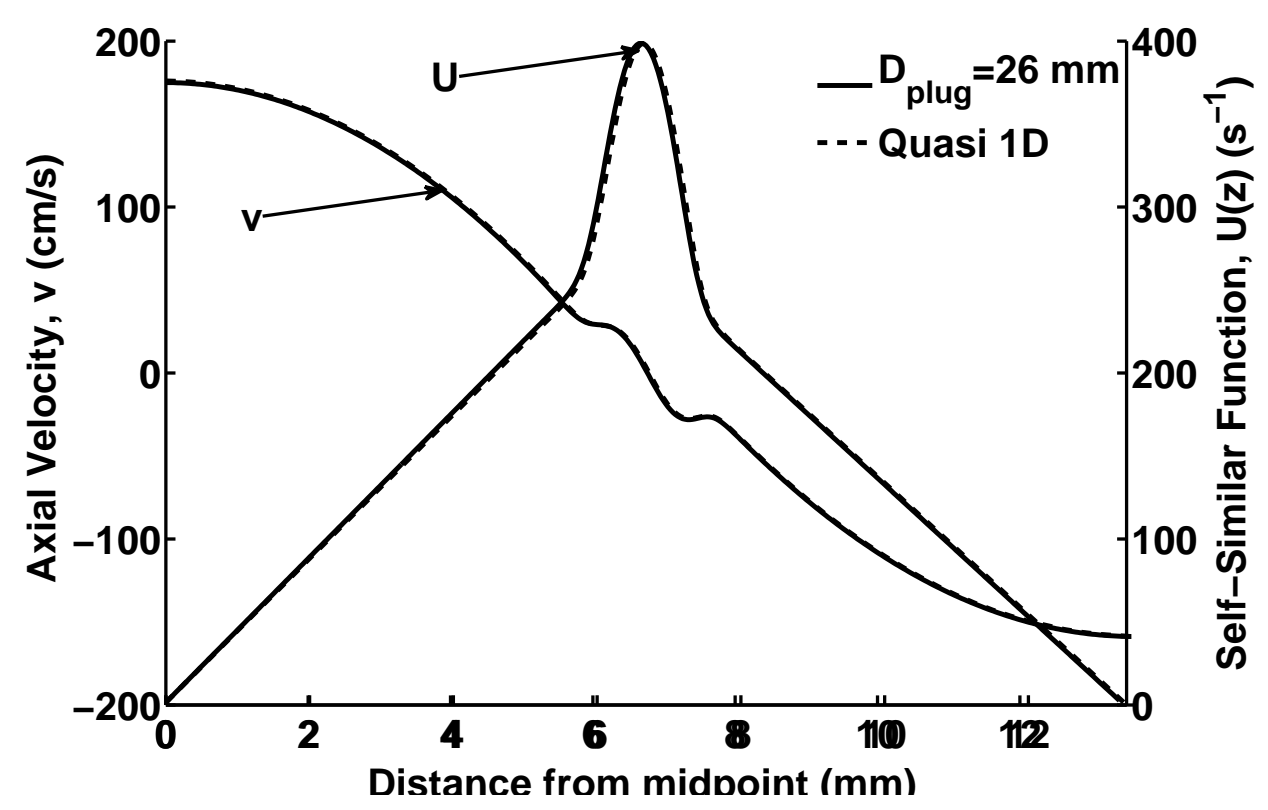

Figure 3: Comparison of axial velocity and self-similarity function $(U(z))$ using axisymmetric (OpenFOAM - solid line) and quasi one-dimensional (dashed line) simulations, for a plug flow case with a diameter of $26 \mathrm{~mm}$ (Seshadri type burner) and local strain rate of $a=375 \mathrm{~s}^{-1}$. 


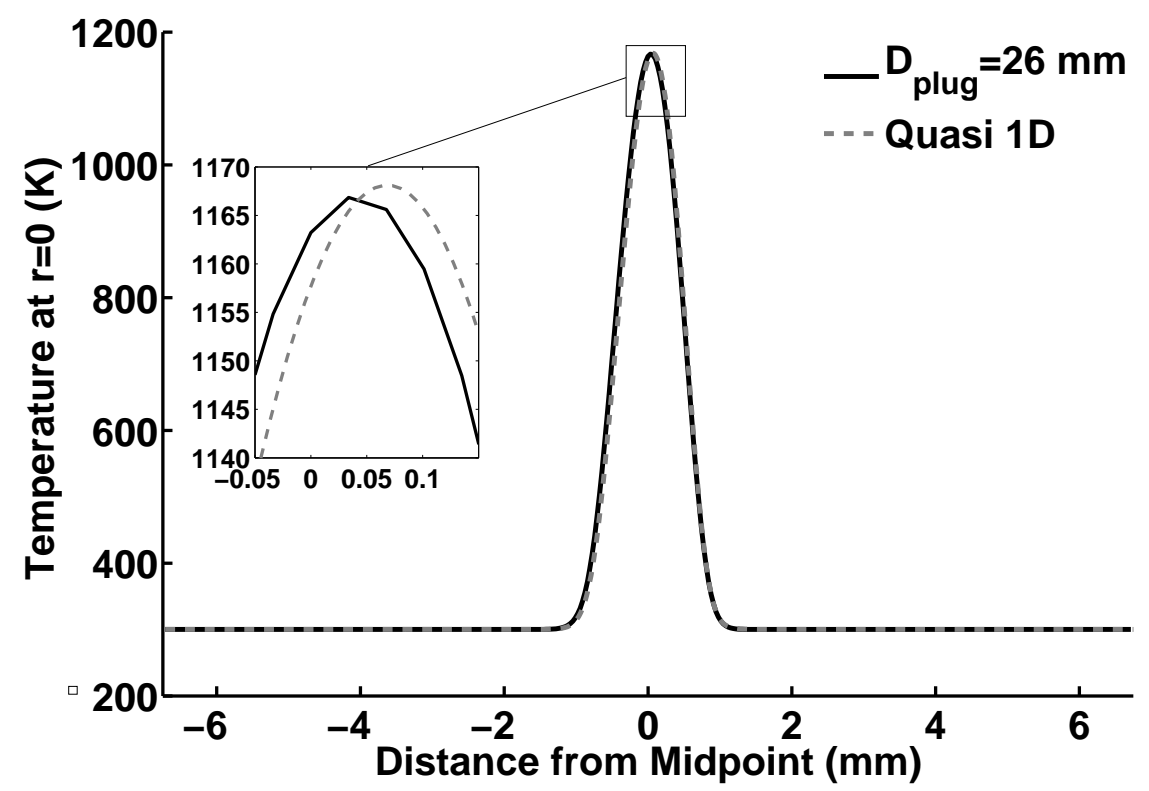

Figure 4: Comparison of flame temperature using axisymmetric (OpenFOAM - solid line) and quasi one-dimensional (dashed line) simulations, for a plug flow case with a diameter of $26 \mathrm{~mm}$ (Seshadri type burner) and strain rate of $a=375 \mathrm{~s}^{-1}$. 


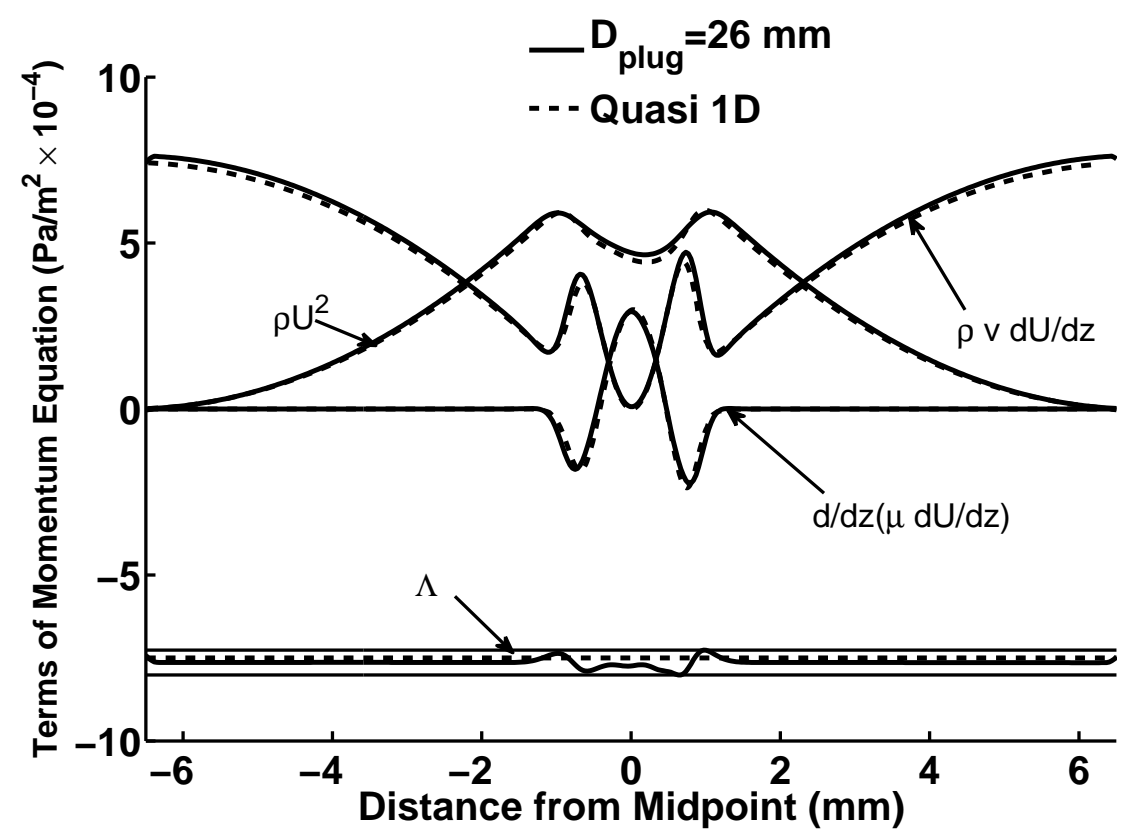

Figure 5: Comparison of the terms in radial momentum equation (including eigenvalue, $\Lambda)$ ) using axisymmetric (OpenFOAM - solid line) and quasi onedimensional (dashed line) simulations, for a plug flow with a diameter of 26 $\mathrm{mm}$ (Seshadri type burner) and strain rate of $a=375 \mathrm{~s}^{-1}$. 


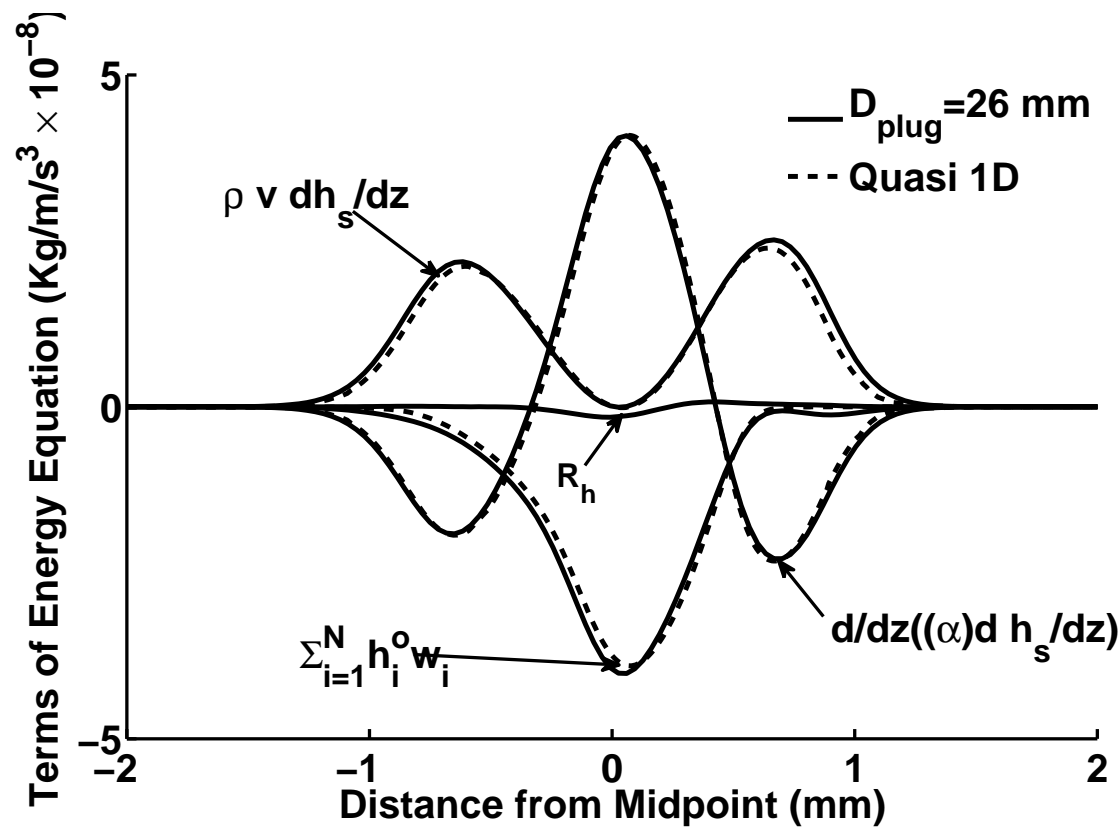

Figure 6: Comparison of the terms in the quasi one-dimensional energy equation using axisymmetric (OpenFOAM - solid line) and quasi one-dimensional (dashed line) simulations, for a plug flow with a diameter of $26 \mathrm{~mm}$ (Seshadri type burner) and strain rate of $a=375 \mathrm{~s}^{-1}$. The residue of axisymmetric solution is identified as $R_{h}$ which is mainly due to the radial heat conduction term. 


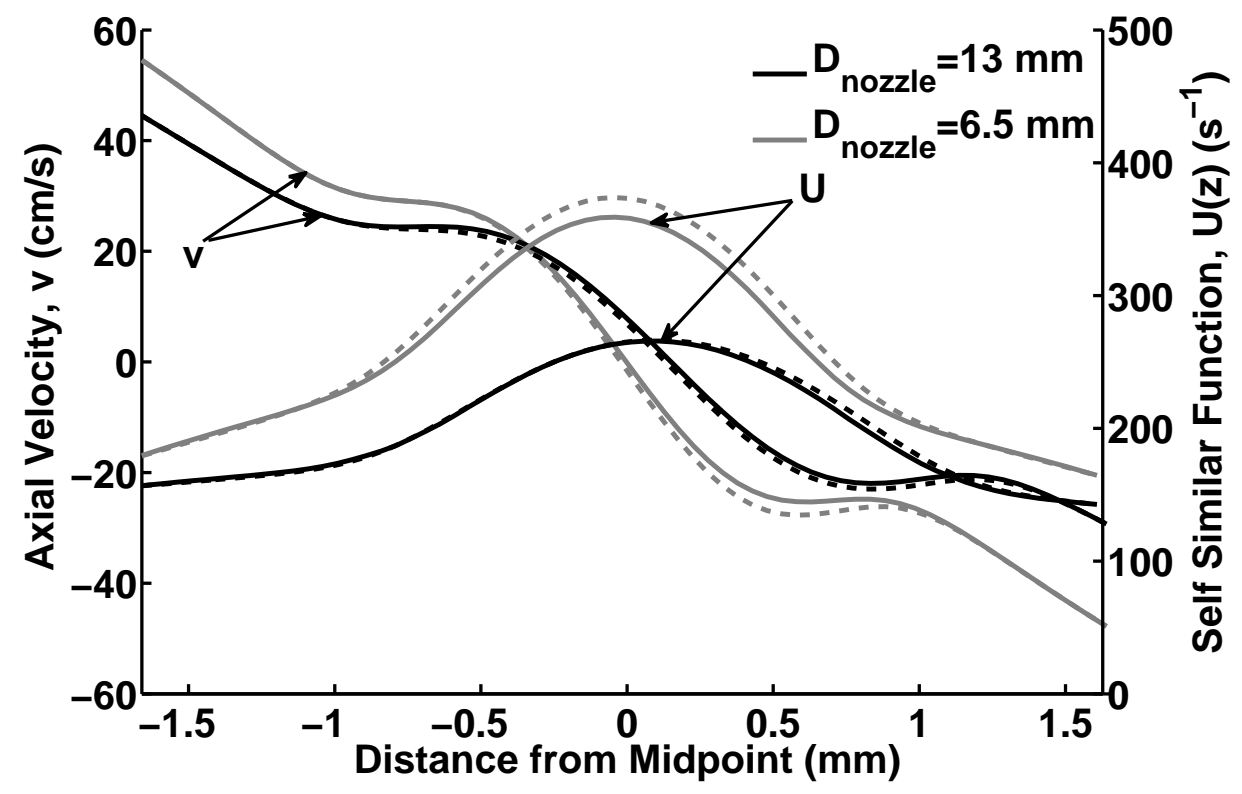

Figure 7: Comparison of axial velocity and self-similarity function $(U(z))$ using axisymmetric (OpenFOAM -solid line) and quasi one-dimensional (dashed line) simulations, for (i) nozzle flow with diameter of $13 \mathrm{~mm}$ and strain rate of $a=268 \mathrm{~s}^{-1}$, and (ii) nozzle flow with diameter of $6.5 \mathrm{~mm}$ and strain rate of $a=354 \mathrm{~s}^{-1}$. 


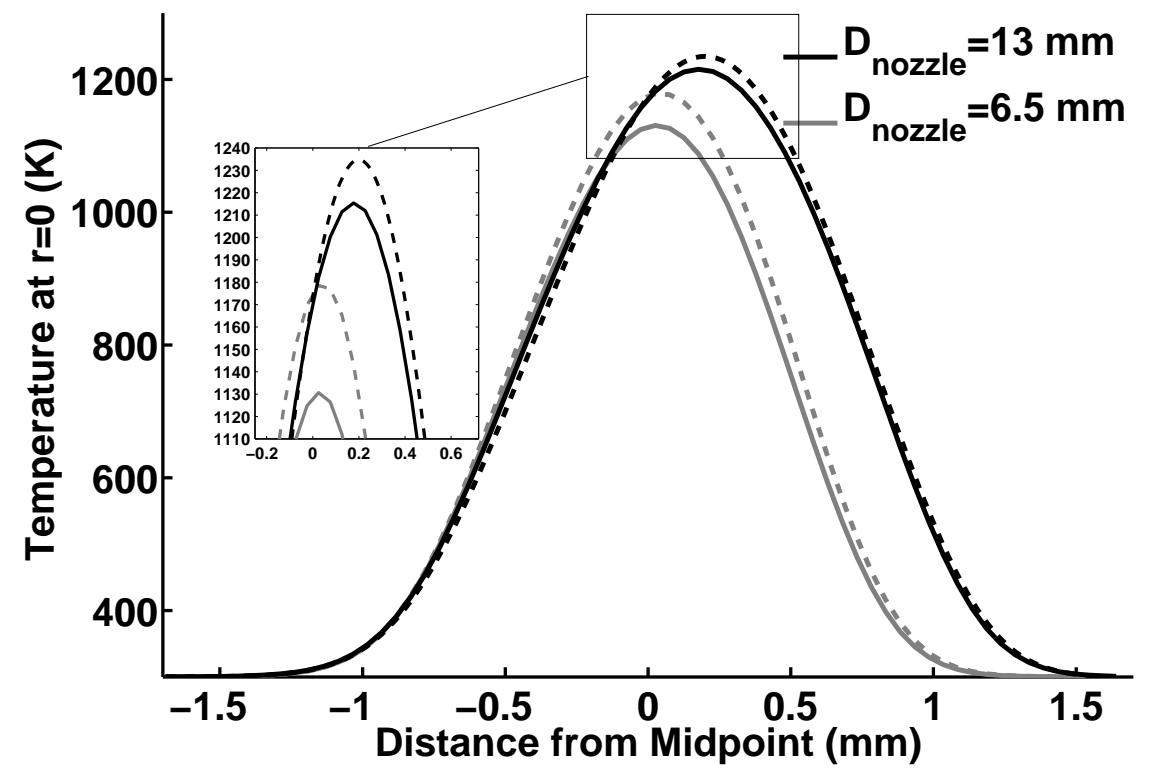

Figure 8: Comparison of flame temperature using axisymmetric (OpenFOAM - solid line) and quasi one-dimensional (dashed line) simulations, for (i) nozzle flow with diameter of $13 \mathrm{~mm}$ and strain rate of $a=268 \mathrm{~s}^{-1}$ and (ii) nozzle flow with diameter of $6.5 \mathrm{~mm}$ and strain rate of $a=354 \mathrm{~s}^{-1}$. 


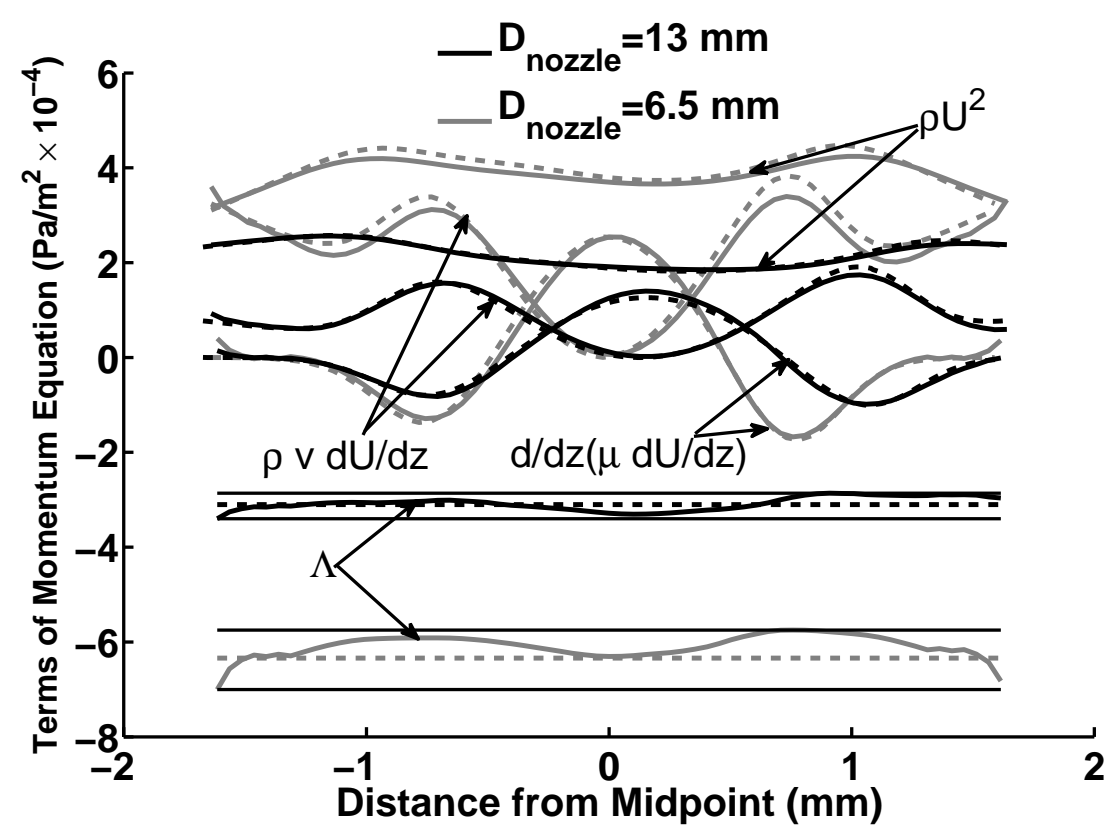

Figure 9: Comparison of the terms in radial momentum equation (including eigenvalue, $\Lambda$ )) using axisymmetric (OpenFOAM - solid line) and quasi onedimensional (dashed line) simulations, for (i) nozzle flow with diameter of 13 $\mathrm{mm}$ and strain rate of $a=268 \mathrm{~s}^{-1}$ and (ii) nozzle flow with diameter of 6.5 $\mathrm{mm}$ and strain rate of $a=354 \mathrm{~s}^{-1}$. 


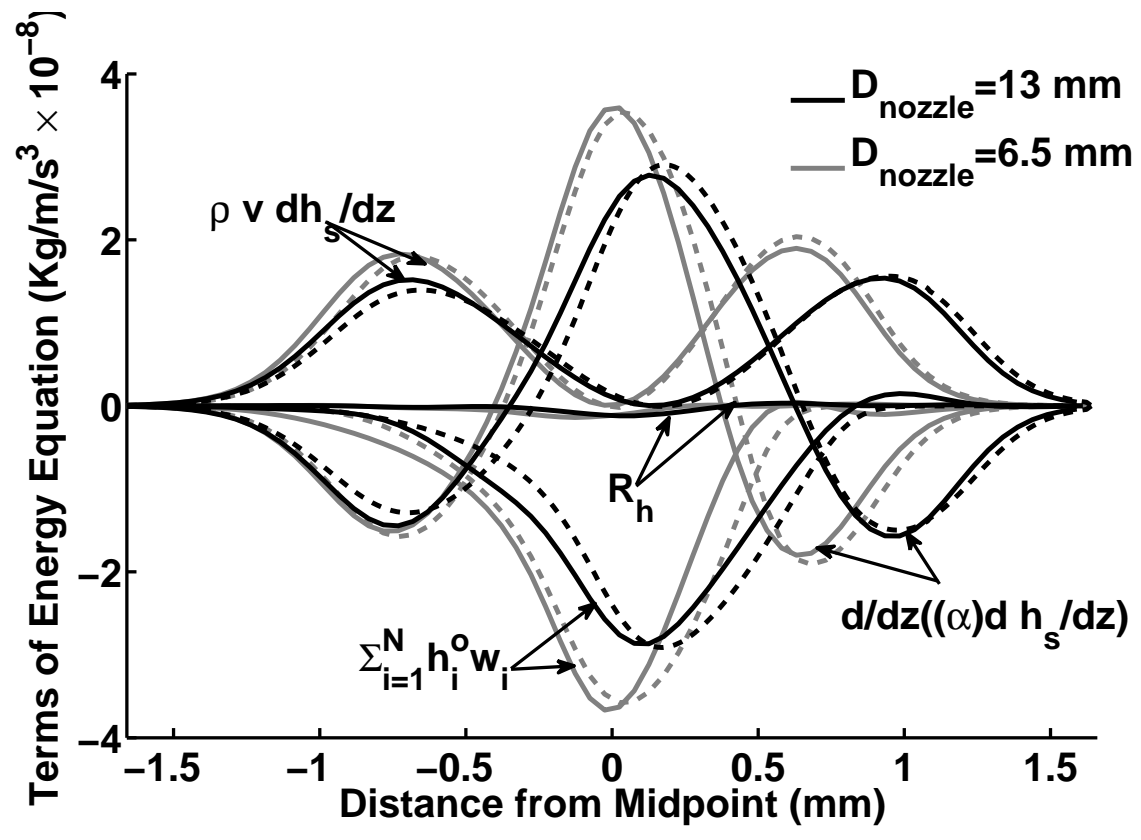

Figure 10: Comparison of the terms in the quasi one-dimensional energy equation using axisymmetric (OpenFOAM - solid line) and quasi onedimensional (dashed line) simulations, for (i) nozzle flow with diameter of 13 $\mathrm{mm}$ and strain rate of $a=268 \mathrm{~s}^{-1}$ and (ii) nozzle flow with diameter of 13 $\mathrm{mm}$ and strain rate of $a=354 \mathrm{~s}^{-1}$. The residue of axisymmetric solution is identified as $R_{h}$ which is mainly due to the radial heat conduction term. 


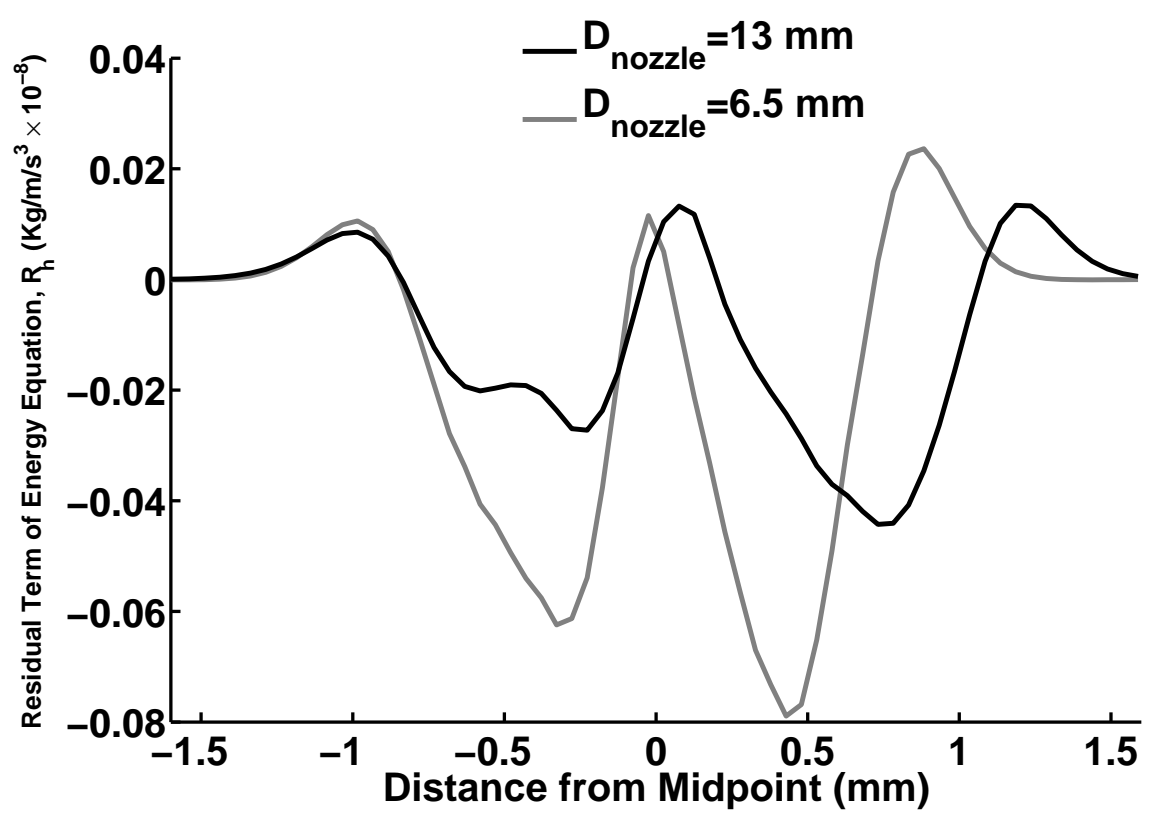

Figure 11: Comparison of the residue term only in the energy equation $\left(R_{h}\right)$, for (i) nozzle flow with diameter of $13 \mathrm{~mm}$ and strain rate of $a=268 \mathrm{~s}^{-1}$ and (ii) nozzle flow with diameter of $13 \mathrm{~mm}$ and strain rate of $a=354 \mathrm{~s}^{-1}$. 


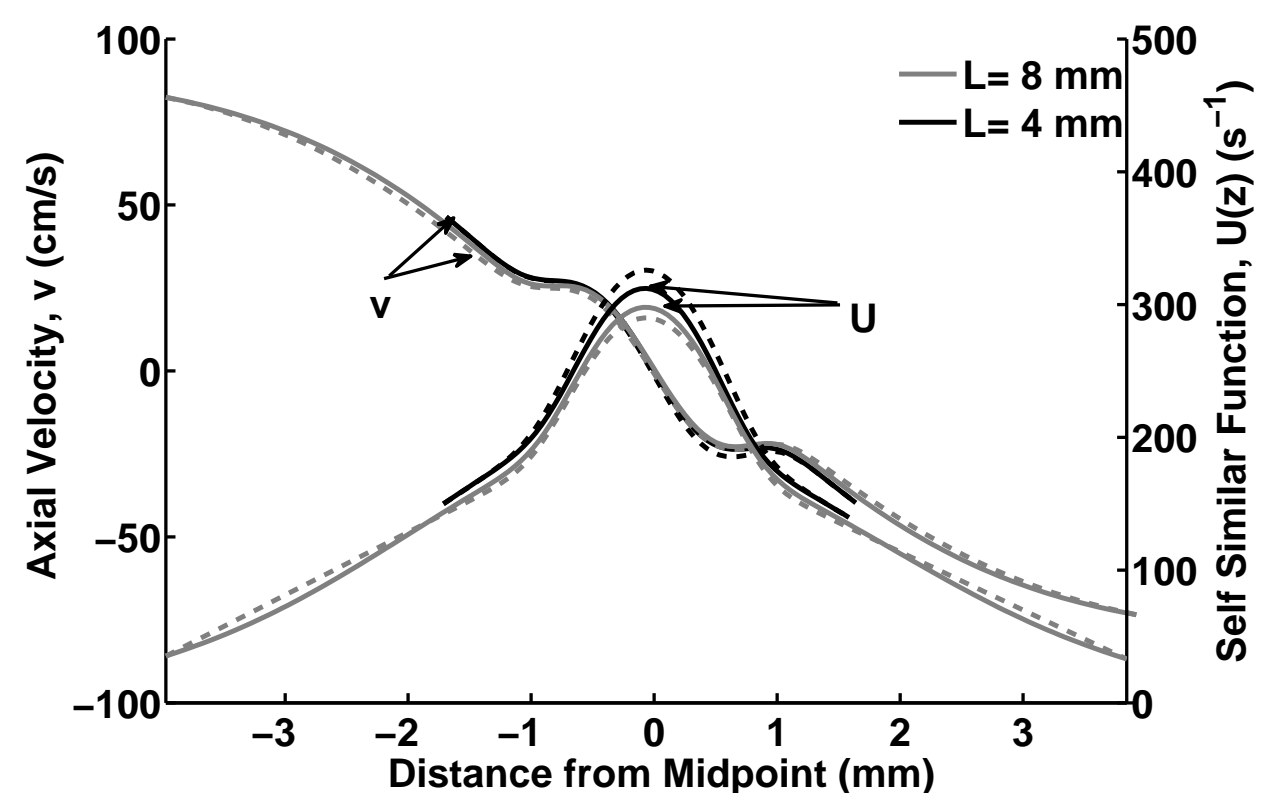

Figure 12: Comparison of axial velocity and self-similarity function $(U(z))$ using axisymmetric (OpenFOAM - solid line) and quasi one-dimensional (dashed line) simulations, for two separation distances using $6.5 \mathrm{~mm} \mathrm{noz-}$ zles (i) $L=8 \mathrm{~mm}$ and strain rate of $a=290 \mathrm{~s}^{-1}$ and (ii) $L=4 \mathrm{~mm}$ and strain rate of $a=354 \mathrm{~s}^{-1}$. 


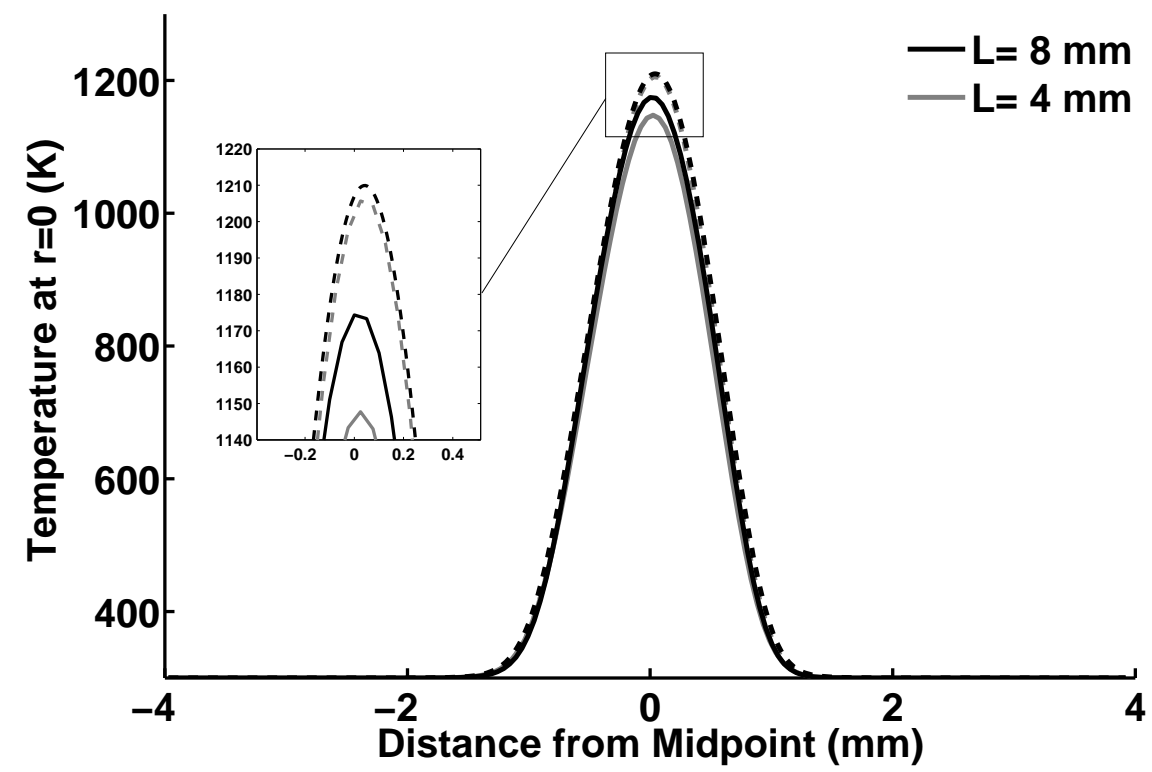

Figure 13: Comparison of temperature using axisymmetric (OpenFOAM solid line) and quasi one-dimensional (dashed line) simulations, for two separation distances using $6.5 \mathrm{~mm}$ nozzles (i) $L=8 \mathrm{~mm}$ and strain rate of $a=290$ $\mathrm{s}^{-1}$ and (ii) $L=4 \mathrm{~mm}$ and strain rate of $a=354 \mathrm{~s}^{-1}$. 


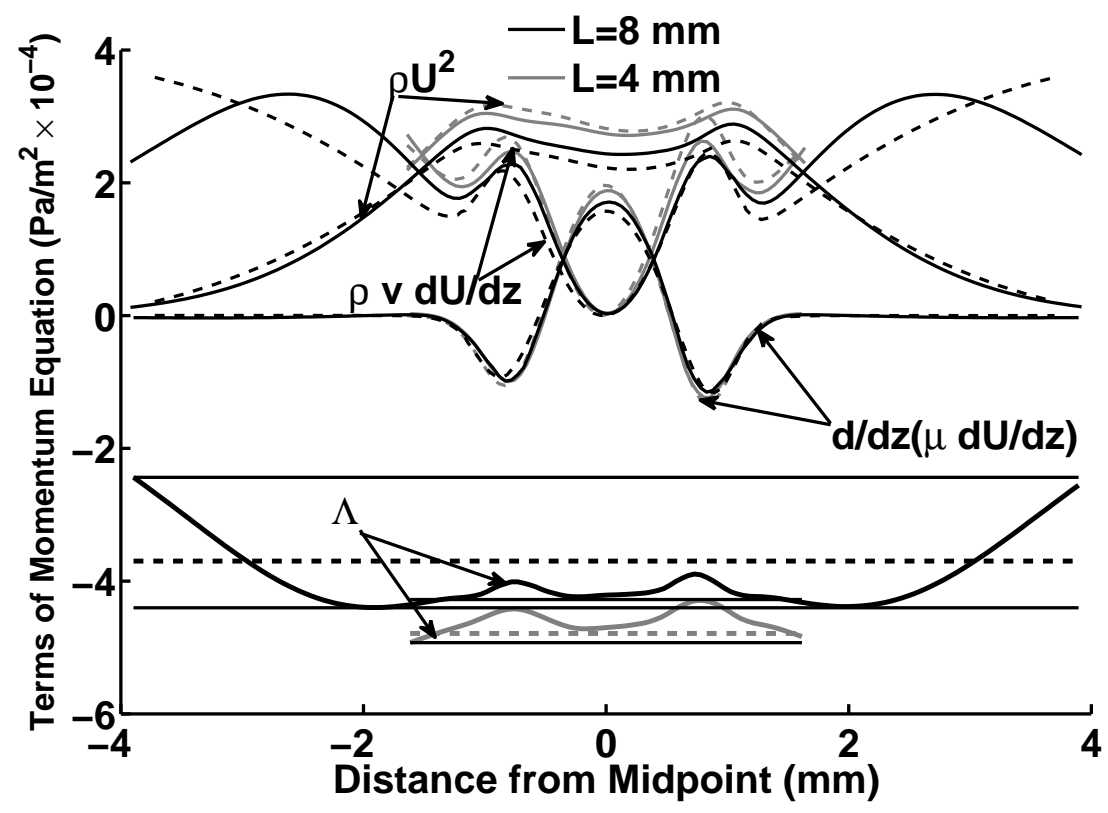

Figure 14: Comparison of the terms in radial momentum equation (including eigenvalue, $\Lambda$ ) using axisymmetric (OpenFOAM -solid line) and quasi onedimensional (dashed line) simulations, for two separation distances using 6.5 mm nozzles (i) $L=8 \mathrm{~mm}$ and strain rate of $a=290 \mathrm{~s}^{-1}$ and (ii) $L=4 \mathrm{~mm}$ and strain rate of $a=354 \mathrm{~s}^{-1}$. 


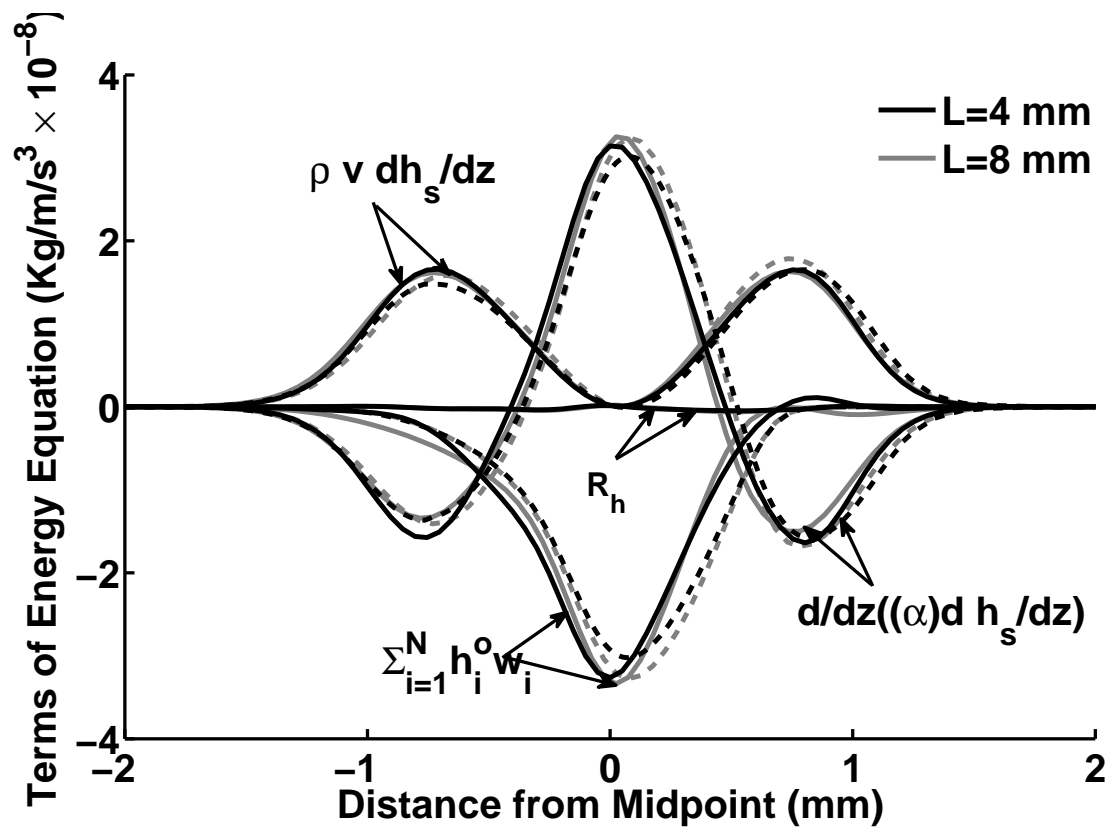

Figure 15: Comparison of the terms in quasi one-dimensional energy equation using axisymmetric (OpenFOAM - solid line) and quasi one-dimensional (dashed line) simulations, for two separation distances using $6.5 \mathrm{~mm}$ nozzles (i) $L=8 \mathrm{~mm}$ and strain rate of $a=290 \mathrm{~s}^{-1}$ and (ii) $L=4 \mathrm{~mm}$ and strain rate of $a=354 \mathrm{~s}^{-1}$. The residue of axisymmetric solution is identified as $R_{h}$ which is mainly due to the radial heat conduction term. 


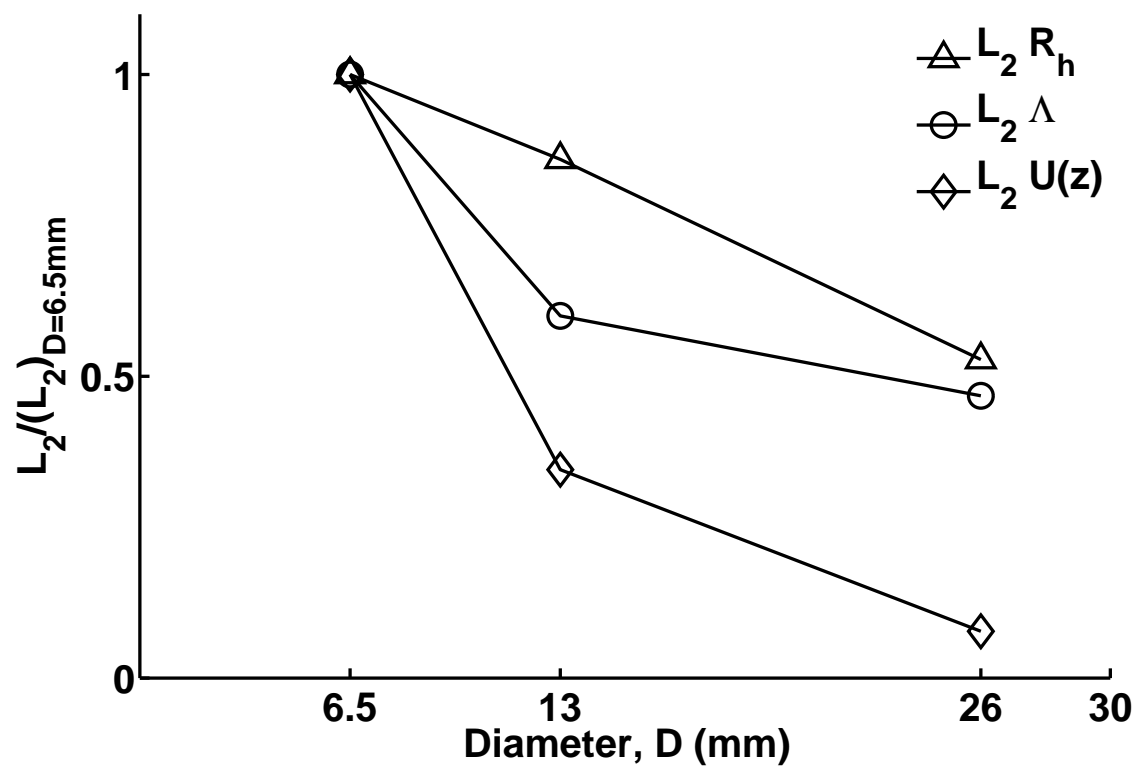

Figure 16: Comparison of the $L_{2}$-norm evaluated based on $\Lambda, R_{h}$, and $U$ as a function of the nozzle diameter, for nozzle separation distance of $L=4 \mathrm{~mm}$. Note: $L_{2}$-norm values are normalized by those at $D=6.5 \mathrm{~mm}$. 


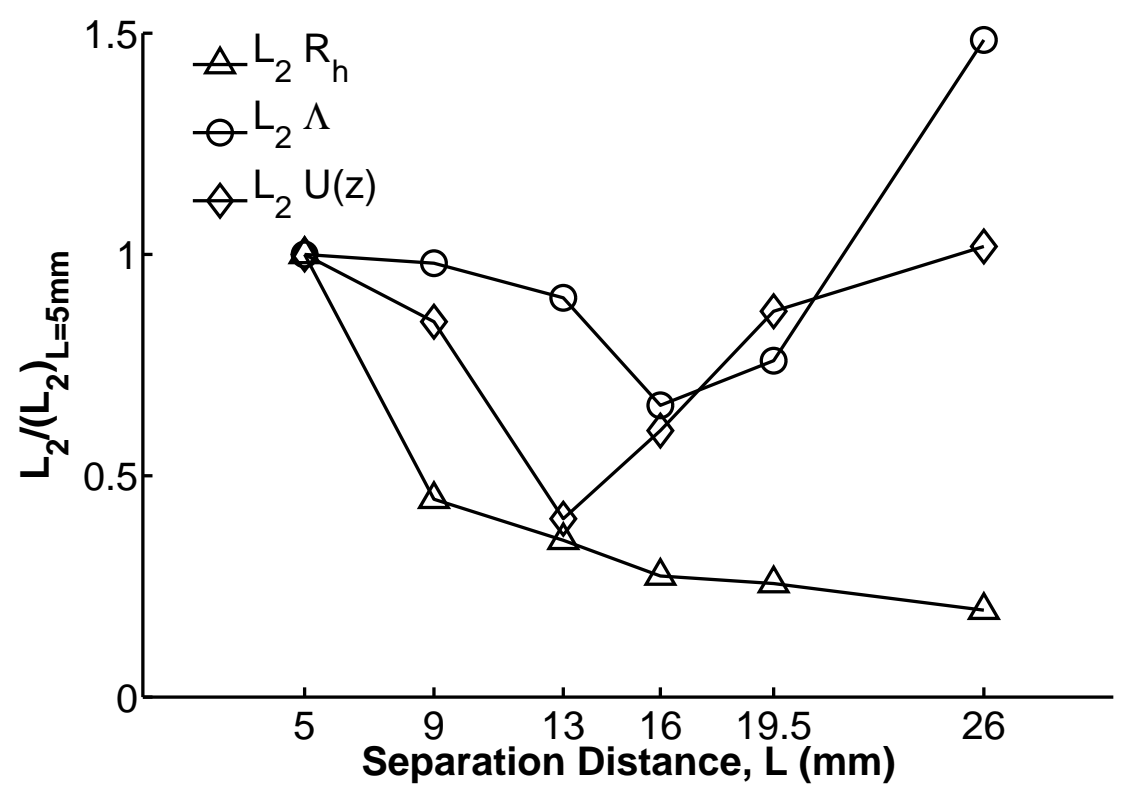

Figure 17: Comparison of the $L_{2}$-norm evaluated based on $\Lambda, R_{h}$, and $U$ as a function of the nozzle separation distance, for nozzle diameter of $D=13 \mathrm{~mm}$. Note: $L_{2}$-norm values are normalized by those at $L=5 \mathrm{~mm}$. 


\section{A Appendix: Verification of OpenFAOM Nu- merical Solver}

The OpenFOAM computational package was used to integrate the following reacting Navier-Stokes Equations,

$$
\begin{aligned}
\frac{\partial \rho}{\partial t}+\nabla \cdot \rho \vec{v} & =0 \\
\frac{\partial \rho \vec{v}}{\partial t}+\nabla \cdot(\rho \vec{v} \vec{v}) & =-\nabla p+\nabla \cdot \mathbb{T}, \\
\frac{\partial \rho h_{s}}{\partial t}+\nabla \cdot \rho \vec{v} h_{s}-\nabla \cdot \rho \alpha \nabla h_{s}+\nabla \cdot \sum_{i=1}^{N} \rho h_{i}^{s} \vec{V}_{i} & =-\sum_{i=1}^{N} h_{i}^{0} w_{i}, \\
\frac{\partial \rho Y_{i}}{\partial t}+\nabla \cdot \rho \vec{v} Y_{i}-\nabla \cdot \rho D_{i} \nabla Y_{i} & =w_{i}, \quad i=1, \ldots, N
\end{aligned}
$$

where $\rho$ is the density, $\vec{v}$ the velocity vector, $p$ the pressure, $\mathbb{T}$ the deviatoric stress tensor, $h_{s}$ the sensible enthalpy, and $\alpha$ the thermal diffusivity of the mixture. For species $i, Y_{i}$ is the mass fraction, $D_{i}$ the mixture average diffusion coefficient, $\vec{V}_{i}$ the mixture average diffusion velocity, $h_{i}^{0}$ the chemical

enthalpy, $h_{i}^{s}$ the sensible enthalpy, and $w_{i}$ the mass production rate. The conservation equations were solved in a segregated manner using secondorder accurate total variation diminishing (TVD) Van-Leer schemes. The finite volume equations were integrated in time using the first-order implicit, Eulerian method. Each equation is solved by iterating until the $L_{2}$-norm of the residual to all equations were less than $10^{-9}$. Simulations were run until steady state was reached. For the purpose of this work, steady state conditions were reached when the norms of all residuals were stationary.

There is no computationally efficient equation that can be used to solve for the pressure field of low Mach number reacting flows. To calculate pressure, the work presented here used the pressure implicit splitting of operators (PISO) method (Issa (1986)), which iterates between the solved velocity field and a guessed pressure distribution until a specified numerical tolerance is met. This pressure solving technique is computationally efficient and has precedence in the combustion community (see Refs. Reinelt, Laurs \& Adomeit (1998); Oevermann, Gerber \& Behrendt (2009); Cuoci, Frassoldati, Faravelli \& Ranzi (2013)).

The sections that follow cover in detail the verification of the OpenFOAM solver via comparison of the $2 \mathrm{D}$ solutions with a set of canonical combustion 
cases simulations.

\section{A.1 Chemistry source term integration}

A direct comparison study was performed in order to verify the correct time integration of chemical source terms by the OpenFOAM solver. The ignition of a homogeneous mixture at constant pressure and enthalpy was simulated with initial mole fraction of $X_{H_{2}}=0.17, X_{O_{2}}=0.17$, and $X_{N_{2}}=0.66$. This homogeneous combustion case was verified against Sandia SENKIN solver (Lutz, Kee \& Miller (1987)) and the results are shown in Figure 18. This was repeated for several different homogeneous mixture concentrations with the results showing the same consistency.

\section{A.2 Premixed one-dimensional reacting flow solution}

A direct comparison study was also performed between the OpenFOAM solver and Sandia one-dimensional premixed flame code (Kee, Grcar, Smooke, Miller \& Meeks (1998)) by considering the spatially varying chemical reactor with a specified constant temperature. The inflow conditions considered were velocity of $2 \mathrm{~m} / \mathrm{s}$, species mole fractions of $X_{H_{2}}=0.004, X_{O_{2}}=0.001$, and $X_{N_{2}}=0.995$, with a constant temperature of $980 \mathrm{~K}$. Pressure was treated as constant at 1 atmosphere in the Sandia code, while the OpenFOAM's PISO method with a far-field pressure node introduced a small pressure departure of the order of $10^{-} 3 \mathrm{~Pa}$ throughout the computational domain. For the above highly diluted case, a one-dimensional mesh was constructed for the OpenFOAM case, with grid spacing in the axial direction of $100 \mu \mathrm{m}$.

Although the species concentrations are small, for the highly reactive hydrogen-oxygen mixture with large species gradients are sensitive to the order of discretization of governing equations. Specifically, for a well resolved case, implementation of central differencing scheme in Sandia onedimensional premix code is found to yield better agreement with the secondorder accurate FVM in OpenFOAM, as shown in Fig. 19. As seen in this figure, the default upwind discretization yields markedly different species profile. 


\section{A.3 Counterflow flame structure and extinction limits}

To verify that the OpenFOAM solver could sufficiently simulate counterflow flame structure and extinction limits, a direct comparison study was performed in comparison to the solution of quasi 1D governing equations using Smooke's code (Smooke et al. (1991)) and Sandia Oppdif Code (Lutz et al. (1997)). It is worth noting that the default discretization of species and energy equations of Sandia and Smooke's codes is upwind for advection terms and central-differencing for diffusion terms. For the same inflow boundary conditions of a diluted hydrogen-air non premixed flame, these two codes resulted in $4 \mathrm{~K}$ difference in the peak flame temperature for a moderately strained flame with a similar grid resolution, which we were unable to explain. However, implementation of a central difference scheme for the advection terms in Smooke's code resulted in $12 \mathrm{~K}$ peak temperature difference. Thus, we believe that the observed peak temperature deviation of 10-15 K between OpenFoam and quasi 1D solvers is likely to be due to numerics. Such temperature differences are expected to influence global flame property comparisons.

Eventhough it is not the main focus of the present paper, the ability to not only predict the flame structure but also predict the extinction limits using the OpenFOAM solver was explored using the Seshadri type flow configuration with a plug-flow velocity profile at the inlets, as shown in Fig. 2a. In this study, the computation domain considered was a smaller 13.5 $\mathrm{mm}$ by $13.5 \mathrm{~mm}$ axisymmetric domain. The non-premixed inflow of fuel and oxidizer streams consisted of diluted hydrogen $\left(X_{H_{2}}=0.16, X_{N_{2}}=0.84\right)$ vs. air $\left(X_{O_{2}}=0.21, X_{N_{2}}=0.79\right)$. The temperature of both inflow streams were assumed to be at $300 \mathrm{~K}$. The outflow boundary condition used a far-field condition for pressure and Neumann condition for all other quantities. The opposed stream flow velocities were gradually increased and convergence was confirmed at strain rates ranging from 200 to $450 \mathrm{~s}^{-1}$ (quasi $1 \mathrm{D}$ solutions predict flame extinction at about $480 \mathrm{~s}^{-1}$ ). For consistency, both simulations used mixture averaged diffusion for species transport, a detailed hydrogen kinetic model extracted from JetSurf2.0 (Wang et al. (2011)), and the same uniform grid resolution of $15 \mu \mathrm{m}$. A comparison of the predicted flame structure for a local strain rate of $365 \mathrm{~s}^{-1}$ is shown in Fig. 20, with comparison to the Smooke's quasi 1D code solution. For the cas shown, the temperature difference is within $2 \mathrm{~K}$. Figure 21 shows a comparison of the predicted peak flame temperature vs. local strain rate, for the same axisymmetric case 
with plug-flow boundary conditions (Seshadri type burner) using OpenFAOM solver and Smooke's quasi one-dimensional code (Smooke et al. (1991)). Numerical error effects in OpenFOAM and the origin of uncertainties shown in Fig. 21 are discussed next.

\section{A.4 Grid resolution effects}

Numerical error and grid dependence effects were analyzed in order to remove the possibility that the general trends highlighted are due to numerical sources. A systematic grid refinement study was examined to determine the grid independence for the OpenFOAM cases. Since the discretization scheme used here was second order accurate, the observed order of accuracy for a response quantity should be close to 2.0. For a reacting case, three consecutive grid refinements yielded observed order of accuracies of 1.84, 1.78, and 1.67 for strain rate, maximum temperature, and $\mathrm{H}$-atom concentrations, respectively. This confirms that the grid choices used in this paper are within the asymptotic range and grid independent. These observed order of accuracies were greater than 1.5 which allowed a factor of safety of 1.5 in the calculation of a grid convergence index (GCI) using Roache's method and Richardson extrapolation (see Roy \& Oberkampf (2010)). This resulted in error estimates of less than $2 \%$ for $\Delta x=15 \mu \mathrm{m}$, for the peak temperature in the counterflow flame simulations that were used to verify the OpenFOAM solver. When the $2 \%$ error applied to temperature obtained from OpenFOAM solver, Fig. 21 shows a predicted flame temperature uncertainty vs. local flow strain rate. 


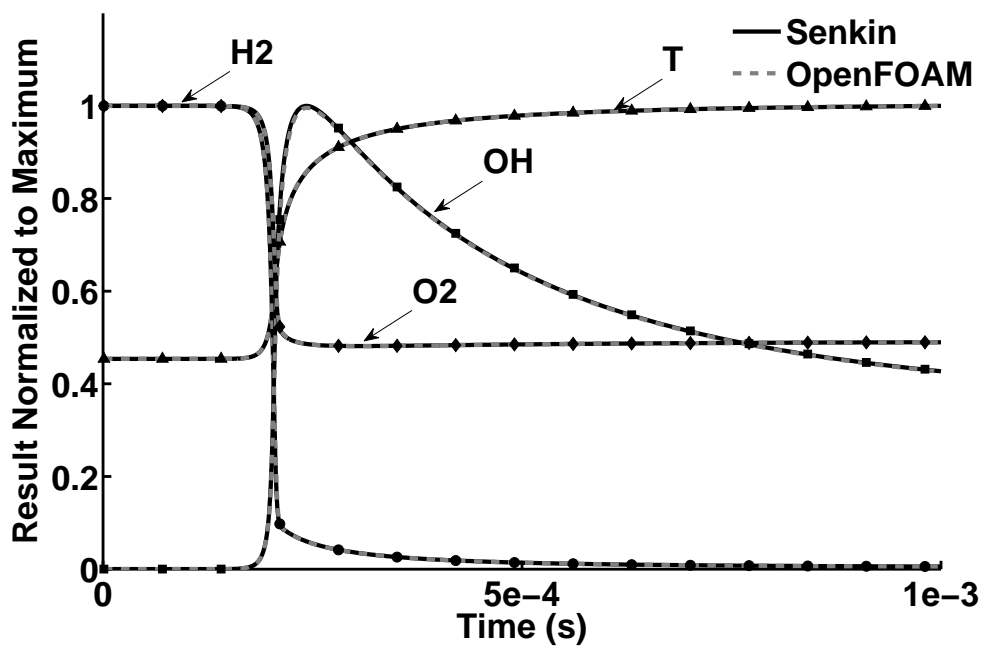

Figure 18: Comparison of temperature and species evolution of a homogeneous mixture at constant pressure and constant enthalpy conditions using OpenFOAM and SENKIN codes. Results are normalized to their maximum values, ie. $T / T_{\max }, X_{H 2} /\left(X_{H 2}\right)_{\max }, X_{O 2} /\left(X_{O 2}\right)_{\max }, X_{O H} /\left(X_{O H}\right)_{\max }$

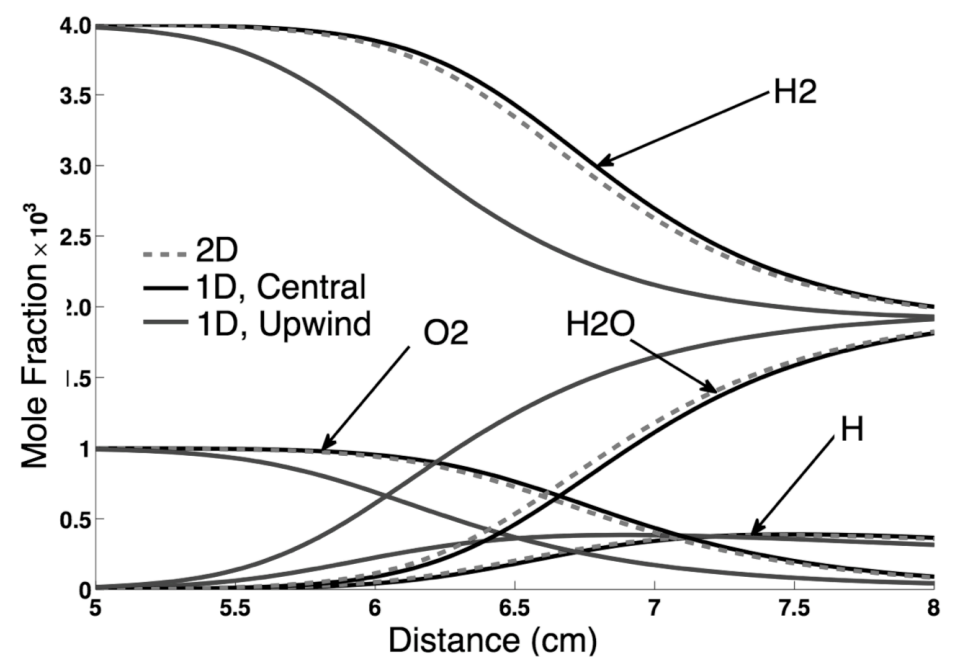

Figure 19: Variation of species mole fractions in a one-dimensional reactor at constant $T=980 \mathrm{~K}$ and $p=1 \mathrm{~atm}$, with inflow conditions of $v=2 \mathrm{~m} / \mathrm{s}, X_{H_{2}}=$ 0.004, $X_{\mathrm{O}_{2}}=0.001$, and $X_{N_{2}}=0.995$. 


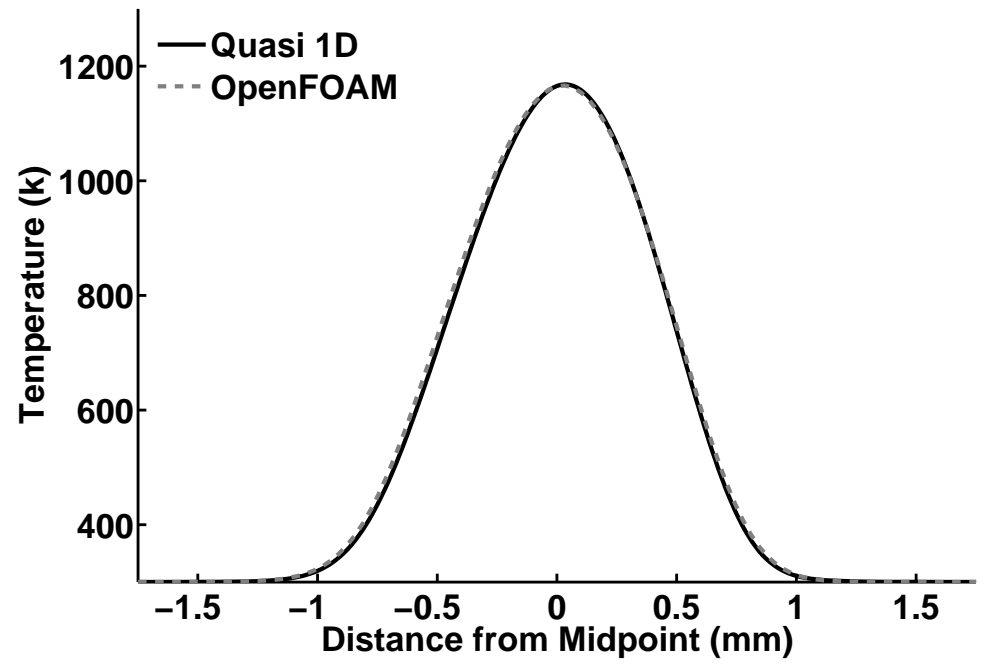

Figure 20: Comparison of the flame temperature profiles predicted using OpenFOAM and quasi one-dimensional codes, for strain rates of $a=375 \mathrm{~s}^{-1}$.

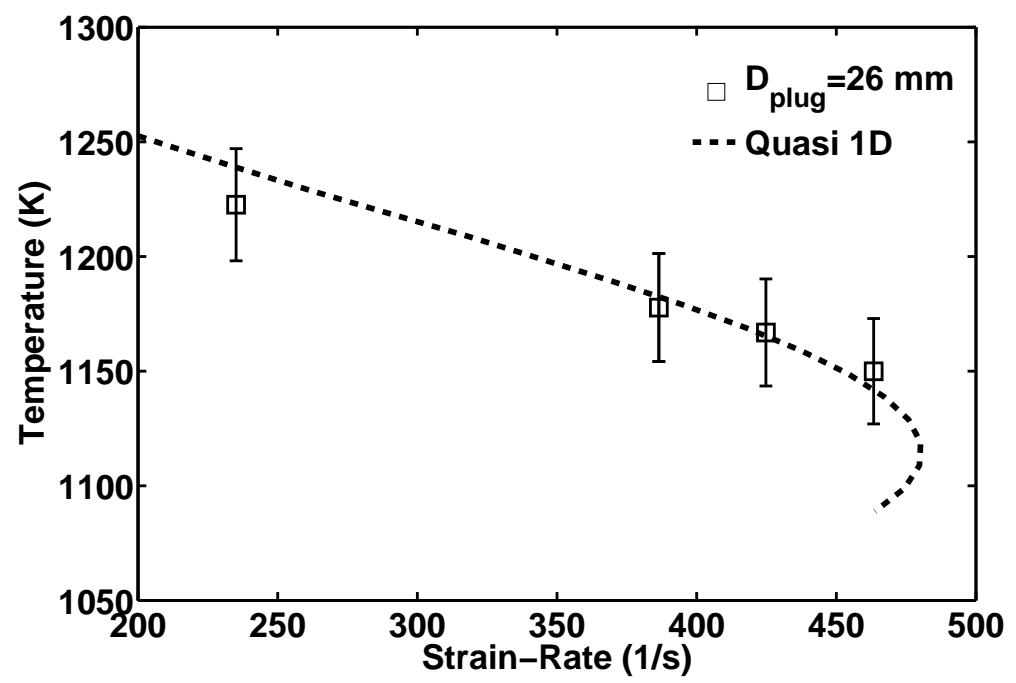

Figure 21: Prediction of maximum flame temperature variation with increasing flow strain rate of a diluted non-premixed hydrogen vs. air flame, using both OpenFOAM and quasi one-dimensional simulations. 


\section{Bibliography}

[1] David K Barton, Daniel Kleppner, Frederick K Lamb, and Maury Tigner. Report of the American Physical Society Study Group on Boost-Phase Intercept Systems for National Missile Defense : Scientific and Technical Issues. 1(October 2004), 2005.

[2] B. Freese. Coal: A Human History. Perseus Pub., 2003.

[3] Nicholas Paduano. Monthly Energy Review: April 2014. page 211, 2014.

[4] R.W. Emerson and B. Atkinson. The Essential Writings of Ralph Waldo Emerson. Modern Library classics. Random House Publishing Group, 2009.

[5] E. T. Turkdogan and J. V. Vinters. Effect of Carbon Monoxide On the Rate of Oxidation of Charcoal, Graphite, and Coke in Carbon Dioxide. Carbon, 8(39-53), 1970.

[6] E T Turkdogan, V. Koump, J V Vinters, and T. F. Perzak. Rate of Oxidation of Graphite in Carbon Dioxide. Carbon, 6:467-484, 1968.

[7] L. G. Overholser and J. P. Blakely. Oxidation of Water of Graphite by Low Concentrations of Carbon Dioxide in Helium. Carbon, 2:385-394, 1965.

[8] J E Antill and K A Peakall. Attack of Graphite by an Oxdising Gas at Low Partial Pressures and High Temperatures. Journal of Nuclear Materials, 1(1):31-38, 1960.

[9] Roald Rindal. An Analysis of the Coupled Chemically Reacting Boundary Layer and Charring Ablator: Part I-VI. NASA Contractor Report, CR-1065(June), 1968.

[10] S.M. Scala and L.M. Gilbert. The Sublimation of Graphite at Hypersonic Speeds. Space Sciences Laboratory, Technical Information Series, Aerophysics Section, (R64SD55), 1964.

[11] Piyush Thakre and Vigor Yang. Chemical Erosion of Carbon-Carbon/Graphite Nozzles in Solid-Propellant Rocket Motors. Journal of Propulsion and Power, 24(4):822833, July 2008.

[12] Piyush Thakre and Vigor Yang. Mitigation of Graphite Nozzle Erosion by BoundaryLayer Control in Solid Propellant Rocket Motors. Journal of Propulsion and Power, 25(5):1079-1085, September 2009. 
[13] Daniele Bianchi, Francesco Nasuti, and Emanuele Martelli. Coupled Analysis of Flow and Surface Ablation in Carbon-Carbon Rocket Nozzles. Journal of Spacecraft and Rockets, 46(3):492-500, May 2009.

[14] Daniele Bianchi, Francesco Nasuti, Marcello Onofri, and Emanuele Martelli. Thermochemical Erosion Analysis for Chraphite/Carbon-Carbon Rocket Nozzles. Journal of Propulsion and Power, 27(1):197-205, January 2011.

[15] J. L. Kassebaum and H. K. Chelliah. Oxidation of Isolated Porous Carbon Particles: Comprehensive Numerical Model. Combustion Theory and Modelling, 13(1):143166, January 2009.

[16] Balaji Venkatachari. Computational Tools for Re-entry Aerothermodynamics; Part II. Surface Ablation. AIAA, (2008-1218):1-12, 2008.

[17] Hugo S Caram and Neal R Amundson. Diffusion and Reaction in a Stagnant Boundary Layer about a Carbon Particle. 16(2):171-181, 1977.

[18] D. Reinelt, A. Laurs, and G. Adomeit. Ignition and Combustion of a Packed Bed in a Stagnation Point Flow Part II: Heterogeneous and Homogeneous Reactions. 2180(97):373-379, 1998.

[19] CW Lau and Stephen Niksa. Models for the Combustion of Individual Particles of Various Coal Types. Preprints of Papers-American Chemicaz Society, pages 13411400, 1993.

[20] Atsushi Makino, Masahiro Hojo, and Masahito Shintomi. Combustion Rate of Solid Carbon in the Axisymmetric Stagnation Flowfield Established over a Sphere and/or a Flat Plate. Journal of Combustion, 2013(4):1-22, 2013.

[21] R. E. Mitchell, J. B. Waters, R. G. Squires, and N. M. Laurendeau. Evidence for Formation of $\mathrm{CO} 2$ in the Vicinity of Burning Pulverized Carbon Particles. Combustion and Flame, 74:91-106, 1988.

[22] Reginald E. Mitchell, Robert J. Kee, Peter Glarborg, and Michael E. Coltrin. The effect of co conversion in the boundary layers surrounding pulverized-coal char particles. Symposium (International) on Combustion, 23(1):1169 - 1176, 1991. TwentyThird Symposium (International) on Combustion.

[23] R. H. Essenhigh. Fundamentals of Coal Combustion. Wiley, 1981.

[24] D. Bradley and Graham Dixon-Lewis. The Oxidation of Graphite Powder in Flame Reaction Zones. Twentieth Symposium (International) on Combustion/The Combustion Institute, 1984, 20(1):931-940, 1985.

[25] A Makino, H.K. Chelliah, C. K. Law, I. Kato, and N. Araki. Modeling of graphite oxidation in a stagnation-point flow field using detailed homogeneous and semiglobal heterogeneous mechanisms with comparisons to experiments. Combustion and Flame, 104(4):469-480, March 1996. 
[26] Atsushi Makino. Mass Transfer Related to Heterogeneous Combustion of Solid Carbon in the Forward Stagnation Region - Part 1 - Combustion Rate and Flame Structure. Mass Transfer in Chemical Engineering Processes, 1994.

[27] Atsushi Makino. Mass Transfer Related to Heterogeneous Combustion of Solid Carbon in the Forward Stagnation Region - Part 2 - Combustion Rate in Special Environments. Mass Transfer in Chemical Engineering Processes, 1994.

[28] A Makino. Combustion rates of graphite rods in the forward stagnation field with high-temperature airflow. Combustion and Flame, 132(4):743-753, March 2003.

[29] Atsushi Makino and Norie Umehara. Combustion Rates of Graphite Rods in the Forward Stagnation Field of the High-temperature, Humid Airflow. Proceedings of the Combustion Institute, 31(2):1873-1880, January 2007.

[30] S. Schulze, M. Kestel, P. a. Nikrityuk, and D. Safronov. From Detailed Description of Chemical Reacting Carbon Particles to Subgrid Models for CFD. Oil \& Gas Science and Technology - Revue d'IFP Energies nouvelles, 68(6):1007-1026, April 2013.

[31] H. Schlichting, K. Gersten, and K. Gersten. Boundary-Layer Theory. Physic and astronomy. MacGraw-Hill, 2000.

[32] H L Reed, W S Saric, and D Arnal. Linear stability theory applied to boundary layers. Annual Review of Fluid Mechanics, 28(1):389-428, 1996.

[33] H Tsuji. Counter flow flames. Elventh Symposium (Int.) on Combustion, 1:979, 1957.

[34] K. Seshadri and F. A. Williams. Laminar Flow Between Parallel Plates With Injection of a Reactant at High Reynolds Number, 1978.

[35] Robert J Kee, James A Miller, Gregory H Evans, and Graham Dixon-Lewis. A Computational Model of the Structure and Extinction of Strained, Opposed Flow, Premixed Methane-Air Flames. Symposium (International) on Combustion, 22(1):1479_ 1494, 1989.

[36] M. D. Smooke, J. Crump, K. Seshadri, and V. Giovangigli. Comparison between experimental measurements and numerical calculations of the structure of counterflow, diluted, methane-air, premixed flames, 1990.

[37] R. I. Acosta, K. C. Gross, and G. P. Perram. Mid-infrared imaging fourier transform spectrometry for high power fiber laser irradiated fiberglass composites, 2012.

[38] Henry E. Revercomb, H. Buijs, Hugh B. Howell, D. D. LaPorte, William L. Smith, and L. A. Sromovsky. Radiometric calibration of ir fourier transform spectrometers: solution to a problem with the high-resolution interferometer sounder. Appl. Opt., 27(15):3210-3218, Aug 1988.

[39] R. I. Issa. The computation of compressible and incompressible recirculating flows by a non-iterative implicit scheme. Journal of Computational Physics, 62:66-82, 1986. 
[40] Michael Oevermann, Stephan Gerber, and Frank Behrendt. Euler-Lagrange/DEM Simulation of Wood Gasification in a Bubbling Fluidized Bed Reactor. Particuology, 7(4):307-316, August 2009.

[41] Alberto Cuoci, Alessio Frassoldati, Tiziano Faravelli, and Eliseo Ranzi. A computational tool for the detailed kinetic modeling of laminar flames: Application to C2H4/CH4 coflow flames. Combustion and Flame, 160(5):870-886, May 2013.

[42] A G Tomboulides. Numerical Simulation of Low Mach Number Reactive Flows. Journal of Scientific Computing, 12(2):139-167, 1997.

[43] H. Courant, R., Friedrichs, K., Lewyt. On the Partial Difference Equations of Mathematical Physics. IBM Journal, (March), 1967.

[44] Tae J Kim, Richard A Yetter, and Frederick L Dryer. New results on moist co oxidation: high pressure, high temperature experiments and comprehensive kinetic modeling. In Symposium (international) on combustion, volume 25, pages 759-766. Elsevier, 1994.

[45] R. J. Kee, J. A. Miller, and J. Warnatz. A fortran computer code package for the evaluation of gas-phase multicomponent transport properties. Sandia Report, SAND 86, 1986.

[46] R. J. Kee, J. A. Miller, and T. H. Jefferson. Chemkin ii: A general purpose, problemindependent, trasnportable, fortran chemical kinetics code package. Sandia Report, SAND 89, 1980.

[47] C. Hirsch. Numerical Computation of Internal and External Flows: Fundamentals of numerical discretization. Wiley series in numerical methods in engineering. Wiley, 1991.

[48] Bram van Leer. Towards the ultimate conservative difference scheme. v. a secondorder sequel to godunov's method. Journal of Computational Physics, 32(1):101 136, 1979.

[49] G-C. Zha and E. Bilgen. Numerical solutions of euler equations by using a new flux vector splitting scheme. International Journal for Numerical Methods in Fluids, 17(2):115-144, 1993.

[50] P.L Roe. Approximate riemann solvers, parameter vectors, and difference schemes. Journal of Computational Physics, 43(2):357 - 372, 1981.

[51] F. A. Williams. Combustion Theory: Second Edition. Westview Press, 2nd edition, March 1994.

[52] Richard Haberman. Elementary applied partial differential equations. Prentice Hall Englewood Cliffs, NJ, 1983. 
[53] Vigor Yang, Nasser Darabiha, Guillaume Ribert, Laetitia Pons, Nan Zong, and Sébastien Candel. Counterflow diffusion flames of general fluids: Oxygen/hydrogen mixtures. Combustion and Flame, 154(3):319-330, August 2008.

[54] P. M. Gresho and R. L. Sani. On Pressure Boundary Conditions for the Incompressible Navier-Stokes Equations. Int. Journal for Numerical Methods in Fluids, 7:11111145, April 1987.

[55] J. Anderson. Computational Fluid Dynamics. Computational Fluid Dynamics: The Basics with Applications. McGraw-Hill Education, 1995.

[56] J.H. Ferziger and M. Peric. Computational Methods for Fluid Dynamics. Springer London, Limited, 2002.

[57] A.W. Vreman. The projection method for the incompressible Navier-Stokes equations: The pressure near a no-slip wall. Journal of Computational Physics, 263:353374, April 2014.

[58] M. P. Burke, M. Chaos, Y. Ju, F. L. Dryer, and S. J. Klippenstein. Comprehensive $h_{2}$ $/ \mathrm{O}_{2}$ kinetic model for high-pressure combustion. International Journal of Chemical Kinetics, 44:444-474, 2012.

[59] C. J. Roy and W. L. Oberkampf. Verification and Validation in Scientific Computing. Cambridge University Press, Cambridge, UK, 2010.

[60] H. Jasak, A. Jemcov, and Z. Tukovic. Openfoam: A C++ library for complex physics simulations. International workshop on coupled methods in numerical dynamics, IUC, 2007.

[61] C J Sung, J B Liu, and C K Law. Structural Response of Counterflow Diffusion Flames to Strain Rate Variations. 2180(95):481-492, 1995.

[62] G Pellett. Velocity and Thermal Structure, and Strain-Induced Extinction of 14 to $100 \%$ Hydrogen-Air Counterflow Diffusion Flames. Combustion and Flame, 112(4):575-592, 1998.

[63] C E Frouzakis, J Lee, A G Tomboulides, and K Boulouchos. Two-dimensional direct numerical simulation of opposed-jet hydrogen-air diffusion flame. pages 571-577, 1998.

[64] J. C. Rolon, D. Veynante, J. P. Martin, and E. Durst. Counter jet stagnation flows. Experiments and Fluids, 11:313-324, 1991.

[65] HarshaK. Chelliah, Cesár Treviño, and FormanA. Williams. Asymptotic analysis of methane-air diffusion flames. In MitchellD. Smooke, editor, Reduced Kinetic Mechanisms and Asymptotic Approximations for Methane-Air Flames, volume 384 of Lecture Notes in Physics, pages 137-158. Springer Berlin Heidelberg, 1991. 
[66] Jeffrey M Bergthorson, Kazuo Sone, Trent W Mattner, Paul E Dimotakis, David G Goodwin, and Dan I Meiron. Impinging laminar jets at moderate Reynolds numbers and separation distances. Physical review. E, Statistical, nonlinear, and soft matter physics, 72(6 Pt 2):066307, December 2005.

[67] Jeffrey Bergthorson, Kazuo Sone, Trent Mattner, Paul Dimotakis, David Goodwin, and Dan Meiron. Impinging laminar jets at moderate Reynolds numbers and separation distances. Physical Review E, 72(6):066307, December 2005.

[68] B.G. Sarnacki, G. Esposito, R.H. Krauss, and H.K. Chelliah. Extinction limits and associated uncertainties of nonpremixed counterflow flames of methane, ethylene, propylene and n-butane in air. Combustion and Flame, 159(3):1026-1043, March 2012.

[69] J Lee, C E Frouzakis, and K Boulouchos. Two-dimensional direct numerical simulation of opposed-jet hydrogen / air flames : Transition from a diffusion to an edge flame $\mathrm{n} / \mathrm{a}$ hydrogen / air flames : Transition from a diffusion to an edge flame. 28:801-806, 2000 .

[70] Konstantinos Boulouchos, Ananias G Tomboulides, Jerry Lee, and Christos E Frouzakis. From diffusion to premixed flames in an H2/air opposed-jet burner: the role of edge flames. Combustion and Flame, 130(3):171-184, 2002.

[71] Varun Mittal, Heinz Pitsch, and Fokion Egolfopoulos. Assessment of counterflow to measure laminar burning velocities using direct numerical simulations. Combustion Theory and Modelling, 16(3):419-433, June 2012.

[72] Nicolas Bouvet, Dmitry Davidenko, Christian Chauveau, Laure Pillier, and Youngbin Yoon. On the simulation of laminar strained flames in stagnation flows: 1D and 2D approaches versus experiments. Combustion and Flame, pages 1-15, October 2013.

[73] John C. Duda, Francis D. Lagor, and Amy S. Fleischer. A flow visualization study of the development of vortex structures in a round jet impinging on a flat plate and a cylindrical pedestal. Experimental Thermal and Fluid Science, 32(8):1754-1758, September 2008.

[74] C Cornaro, a.S Fleischer, and R.J Goldstein. Flow visualization of a round jet impinging on cylindrical surfaces. Experimental Thermal and Fluid Science, 20(2):66-78, October 1999.

[75] J M Buchlin. Convective Heat Transfer in Impinging- Gas- Jet Arrangements. 4(2):137-149, 2011.

[76] Anubhav Sinha and Subhash Chander. Numerical investiagtion of flame jet impingement on a flat plate. 2010.

[77] Ahad Ramezanpour, Iraj Mirzaee, David Firth, and Hassan Shirvani. A numerical heat transfer study of slot jet impinging on an inclined plate. International Journal of Numerical Methods for Heat \& Fluid Flow, 17(7):661-676, 2007. 
[78] Shunpei Nakazawa, Takeshi Yokomori, and Masahiko Mizomoto. Flame synthesis of carbon nanotubes in a wall stagnation flow. Chemical Physics Letters, 403(1-3):158162, February 2005.

[79] Jun-bo Huang and Jiin-yuh Jang. Numerical Study of a Confined Axisymmetric Jet Impingement Heat Transfer with Nanofluids. c(January):69-74, 2013.

[80] M. T. Sholtz. and Olev Trass. Mass Transfer in a Nonuniform Impinging Jet. AIChE, pages 82-90, 1970.

[81] Denis J. Phares, Gregory T. Smedley, and Richard C. Flagan. The inviscid impingement of a jet with arbitrary velocity profile. Physics of Fluids, 12(8):2046, 2000.

[82] B. J. Abu-Ghannam and R. Shaw. Natural transition of boundary layers - the effects of turbulence, pressure gradient, and flow history. Journal of Mechanical Engineering Science, 22(5):213-228, 1980.

[83] Q. Brewster. Thermal Radiative Transfer and Properties. A Wiley-Interscience publication. Wiley, 1992.

[84] J Abrahamson. The Energies of Graphite. Carbon, 11(4), 1973. 